\title{
EXPERIMENTAL FACILITIES DIVISION PROGRESS REPORT 1996-97
}

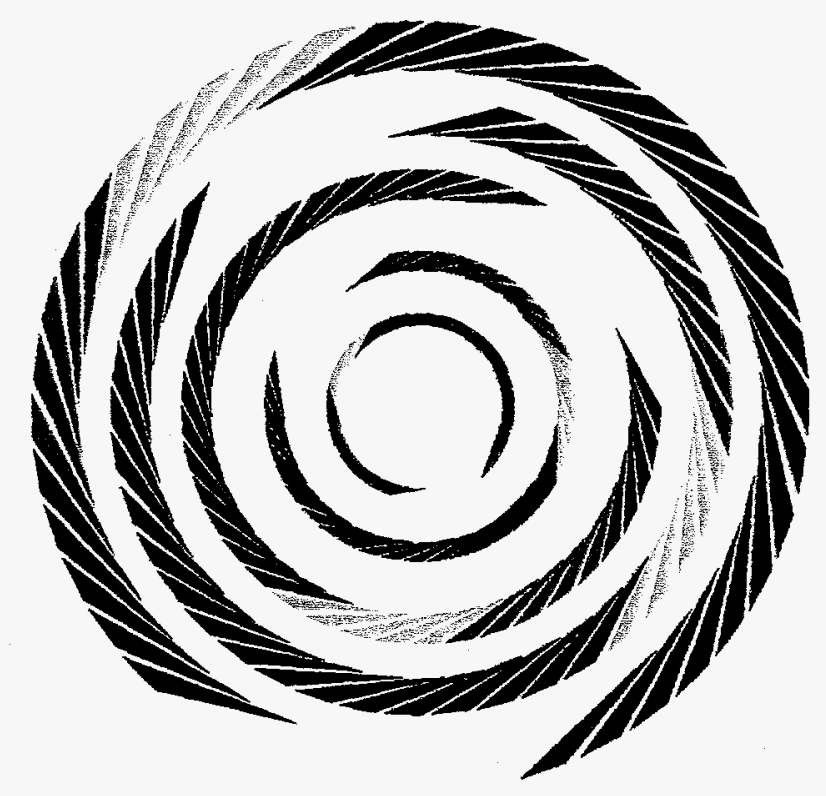

ANL/APS/TB-30

April 1997

ADVANCED PHOTON SOURCE / ARGONNE NATIONAL LABORATORY

Argonne National Laboratory operated by The University of Chicago for the U.S. Department of Energy under Contract W-31-109-Eng-38 


\section{DISCLAIMER}

This report was prepared as an account of work sponsored by an agency of the United States Government. Neither the United States Government nor any agency thereof, nor any of their employees, make any warranty, express or implied, or assumes any legal liabiity or responsibility for the accuracy, completeness, or usefulness of any information, apparatus, product, or process disclosed, or represents that its use would not infringe privately owned rights. Reference herein to any specific commercial product, process, or service by trade name, trademark, manufacturer, or otherwise does not necessarily constitute or imply its endorsement, recommendation, or favoring by the United States Government or any agency thereof. The views and opinions of authors expressed herein do not necessarily state or reflect those of the United States Government or any agency thereof. 


\section{DISCLAIMER}

Portions of this document may be illegible electronic image products. Images are produced from the best available original document. 


\section{Contents}

Abbreviations

vii

\section{OVERVIEW}

1.1 Background

1.2 Mission of the APS Experimental Facilities Division.

1.3 APS User Sector Layout.

1.4 XFD Organization.

1.5 APS User Operations.

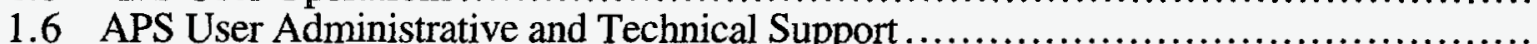

1.7 R\&D in Support of User Operations.

1.8 SRI-CAT.

1.9 Collaborative Work with APS Users and Other Synchrotron Radiation Facilities ..........

1.10 Long-Term Strategic Plan for XFD.

\section{USER OPERATIONS}

2.1 Introduction

2.1.1 Advanced Photon Source Facility Description.

2.1.2 APS Operations

2.1.3 XFD Operations Organization

2.2 Beamline Technical Description

2.2 .1 Introduction

2.2.2 Operational Aspects of IDs.

2.2.3 The Beamline FE Operation.

2.2 .4 Equipment Protection System

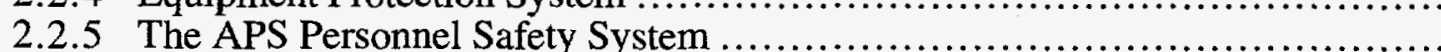

2.2.6 Controls...

2.3 Operations Performance and Reliability

2.3.1 Installation Status

2.3.2 Operations Experience.....

2.3.3 Reliability Studies and Analysis

2.4 User Operations Interface and Support.

2.4.1 Beamline Commissioning Process ............................................

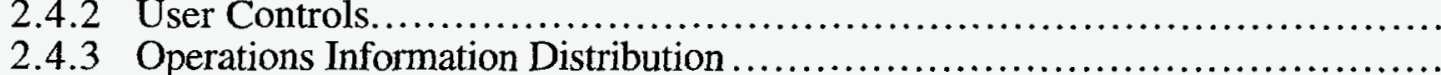

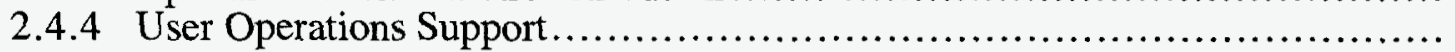

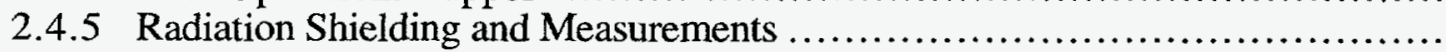

\section{USER ADMINISTRATION AND TECHNICAL SUPPORT}

3.1 User Administration

3.1.1 APS Users.

3.1.2 User Administrative Functions ........................................... 46

3.2 User Safety and Training.......................................................... 50

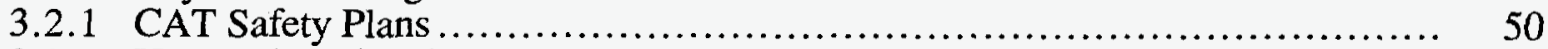

3.2 .2 User Safety Training.......................................................... 51

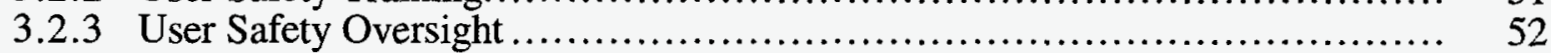

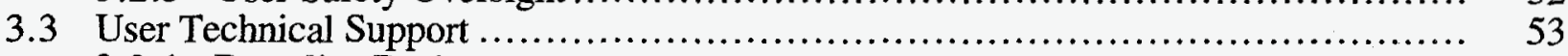

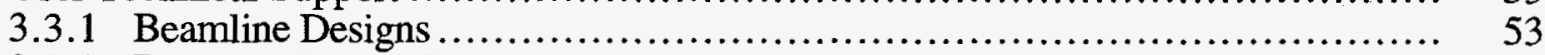

3.3.2 Beamline Design Exchange ................................................. 56 


\section{Contents (Cont.)}

3.3.3 X-ray Optics Metrology and Fabrication .................................. 58

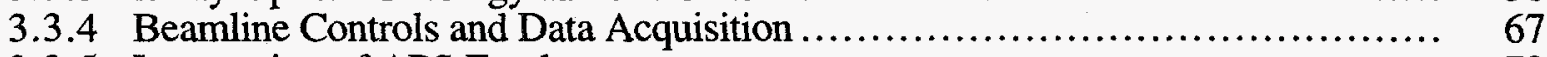

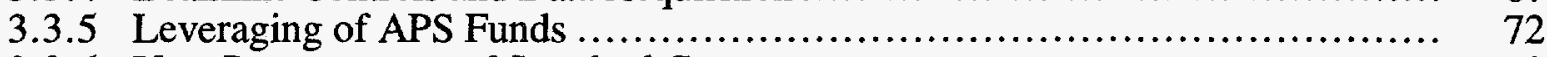

3.3.6 User Procurements of Standard Components .............................. 72

3.3.7 The Inter-CAT Technical Working Group .................................... 74

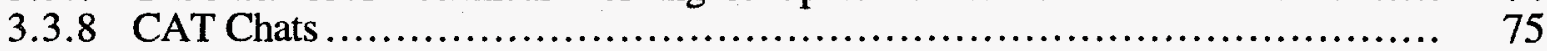

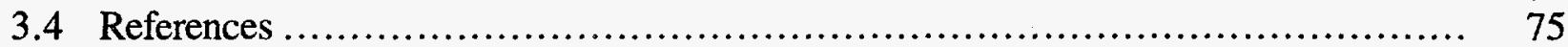

\section{R\&D IN SUPPORT OF USER OPERATIONS}

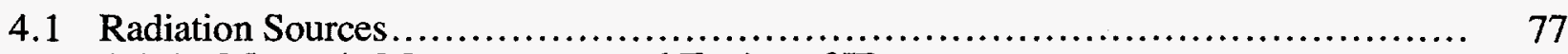

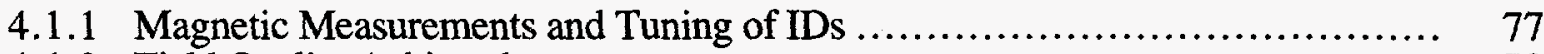

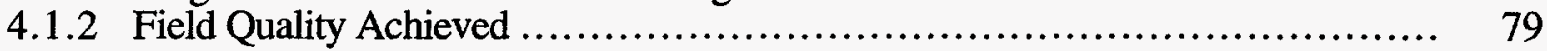

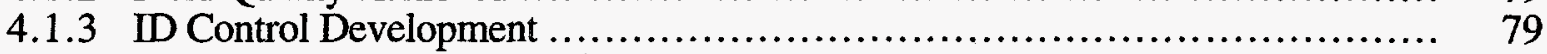

4.1.4 ID Vacuum-Chamber Development ..................................... 82

4.1.5 5-mm-Aperture ID Vacuum Chamber ....................................... 84

4.1.6 EMW Vacuum Chamber Development ....................................... 85

4.1.7 Development of a Novel Vacuum Sealing Technique....................... 87

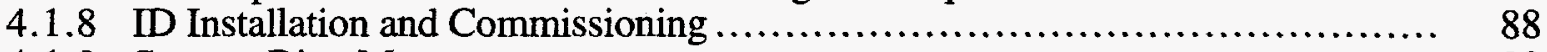

4.1.9 Storage Ring Measurements ................................................ 89

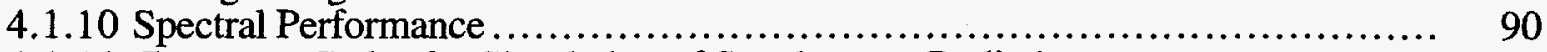

4.1.11 Computer Codes for Simulation of Synchrotron Radiation.................... 92

4.1.12 XOP: A Graphical User Interface for X-ray Optics Utilities.................. 93

4.1.13 UR: Computer Code for Simulation of Real IDs with Real Beams ............ 93

4.1.14 Radiation Exposure of the IDs.......................................... 94

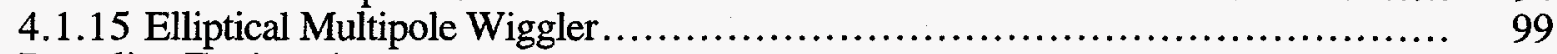

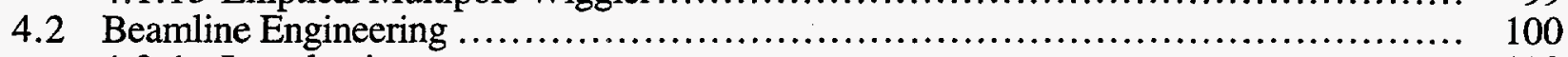

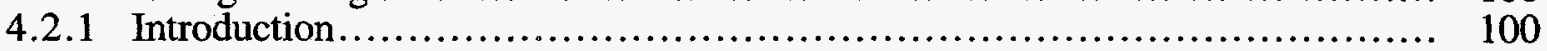

4.2.2 Performance Review of the Important FE and Beamline Components ........... 100

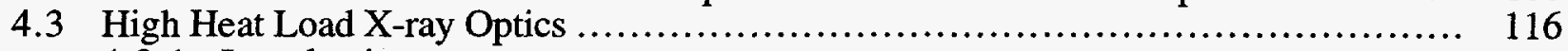

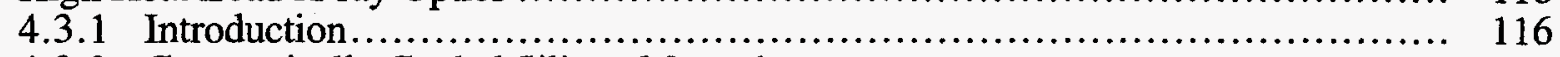

4.3.2 Cryogenically Cooled Silicon Monochromators............................. 117

4.3.3 Liquid-Nitrogen Pumps .............................................. 121

4.3.4 Water-Cooled Diamond Monochromators .................................... 122

4.3.5 Cooled Mirrors ............................................................. 125

4.3.6 Other Work Related to Cooled Optics ................................. 127

4.3.7 The Double-Crystal Monochromator Design ................................. 129

4.3.8 Brilliance/Brightness Measurements..................................... 130

4.4 New Instruments and Techniques............................................... 132

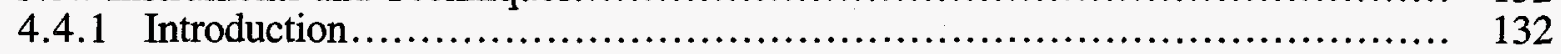

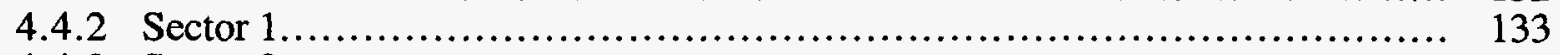

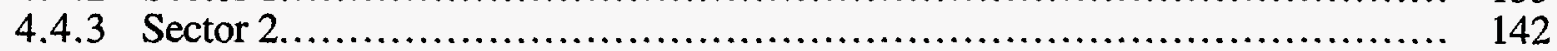

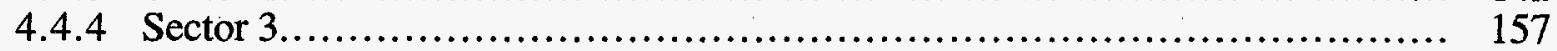

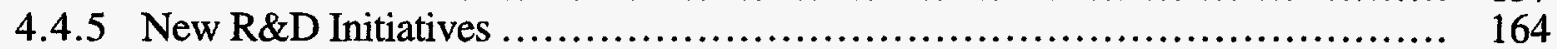

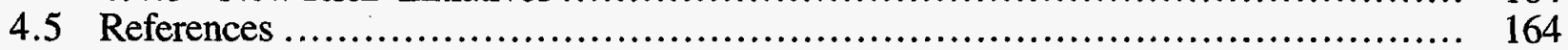

\section{COLLABORATIVE RESEARCH}

5.1 Introduction ................................................................ 169

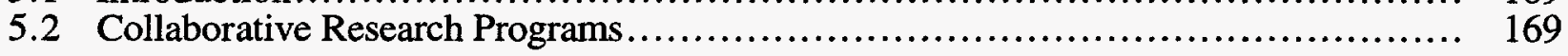




\section{Contents (Cont.)}

5.3 Special Grants for New Collaborative Research .................................. 169

5.4 Collaborative Projects with Other Synchrotron Radiation Facilities ................. 170

\section{LONG-TERM STRATEGIC PLANS FOR XFD}

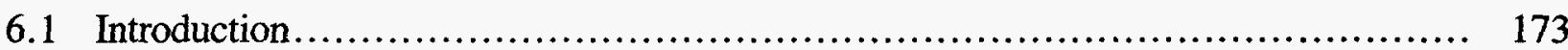

6.2 Accelerator Improvement Program Plans ...................................... 174

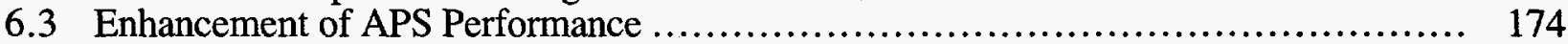

6.4 New Collaborative Access Teams................................................................ 175

6.5 Strategic Plans for Dedicated Beamlines ............................................... 175

6.6 Strategic Plan for FEL Undulator Development................................... 182

6.7 LDRD Program in Support of Long-Term Goals .............................. 182

\section{APPENDICES}

Appendix 1: Recent Publications by XFD Staff................................... 185

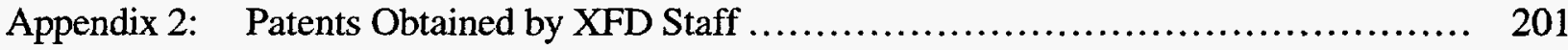

Appendix 3: XFD Primary Web Pages.......................................... 202

Appendix 4: Review Status of APS Collaborative Access Teams as of

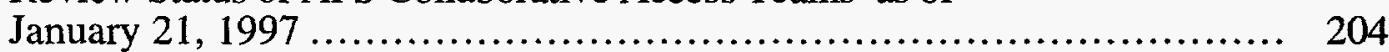

Appendix 5: Bibliography of Publications Produced for APS Users................... 206

Appendix 6: Conferences and Workshops Held at the APS since 1990................ 210

Appendix 7: $\quad$ Outline of the Current APS User Policies and Procedures (3/97) ........... 212

Appendix 8: Metrology Results of Mirrors Delivered and Characterized to Date........... 215

Appendix 9: Invited and Colloquium Talks by XFD Staff......................... 216 


\section{Abbreviations}

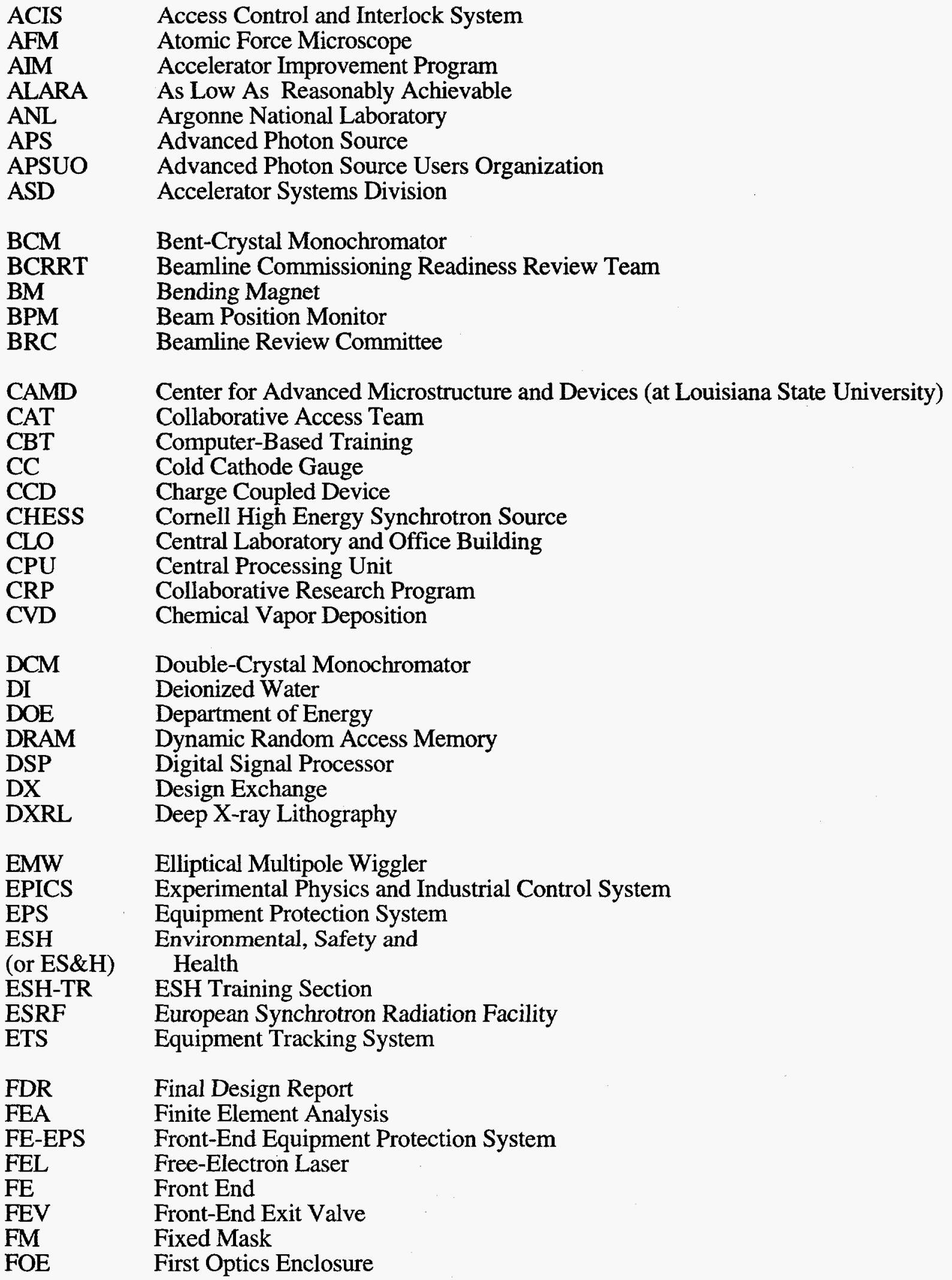


FV

FWHM

GERT

HDF

HVOF

\section{ID \\ IDL \\ IG \\ IOC \\ IR

LDDM
LDRD
LOI
LOM
LTP

MOU

NEG

NSLS

OFHC

OPI

OUI

PAR

PDR

PEB

PLC

PMMA

PS 1

PS2

PSPCD

PSS

R\&D

RD

rf

RGA

SAD

SASE

SAXS

SBPM

SCI

SGM

SPring-8

SRI

SSRL

SV

Fast Valve

Full Width Half Maximum

General Employee Radiation Training

Hierarchical Data Format

High Velocity Oxygen Fuel

Insertion Device

Interactive Data Language

Ion Gauge

Input/Output Controller

Infrared

Laser Doppler Displacement Meter

Laboratory Directed Research \& Development

Letter of Intent

Laboratory/Office Module

Long Trace Profiler

Memorandum of Understanding

Nonevaporable Getter

National Synchrotron Light Source

Oxygen Free High Conductivity

Operator Interface

Operator/User Interface

Positron Accumulator Ring

Preliminary Design Review

Program Evaluation Board

Programmable Logic Controller

Polymethylmethacrylate

Photon Shutter 1

Photon Shutter 2

Position Sensitive Photo-conductive Detector

Personnel Safety System

Research \& Development

Research Directorate

Radio Frequency

Residual Gas Analyzer

Safety Assessment Document

Self Amplification of Stimulated Emission

Small Angle X-ray Scattering

Smart Photon Beam Position Monitor

System Controller Interface

Spherical Grating Monochromator

Super Photon Ring 8 (in Japan)

Synchrotron Radiation Instrumentation

Stanford Synchrotron Radiation Laboratory

Slow Valve 


$\begin{array}{ll}\text { TBPM } & \text { Transmitting X-ray Beam Position Monitor } \\ \text { TLD } & \text { Thermal Luminescent Dosimeter } \\ \text { TTU } & \text { Topo Test Unit } \\ \text { TWG } & \text { Inter-CAT Technical Working Group } \\ \text { UHV } & \text { Ultrahigh Vacuum } \\ \text { URL } & \text { Uniform Resource Locator } \\ \text { UR } & \text { Undulator Radiation } \\ \text { US } & \text { Undulator Spectrum } \\ \text { WBS } & \text { Work Breakdown Structure } \\ \text { XBPM } & \text { X-ray Beam Position Monitor } \\ \text { XFD } & \text { Experimental Facilities Division } \\ \text { XOP } & \text { X-ray Optics Utilities }\end{array}$




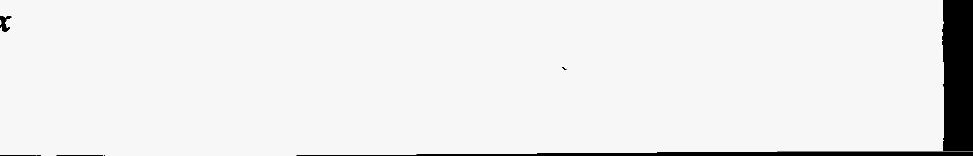




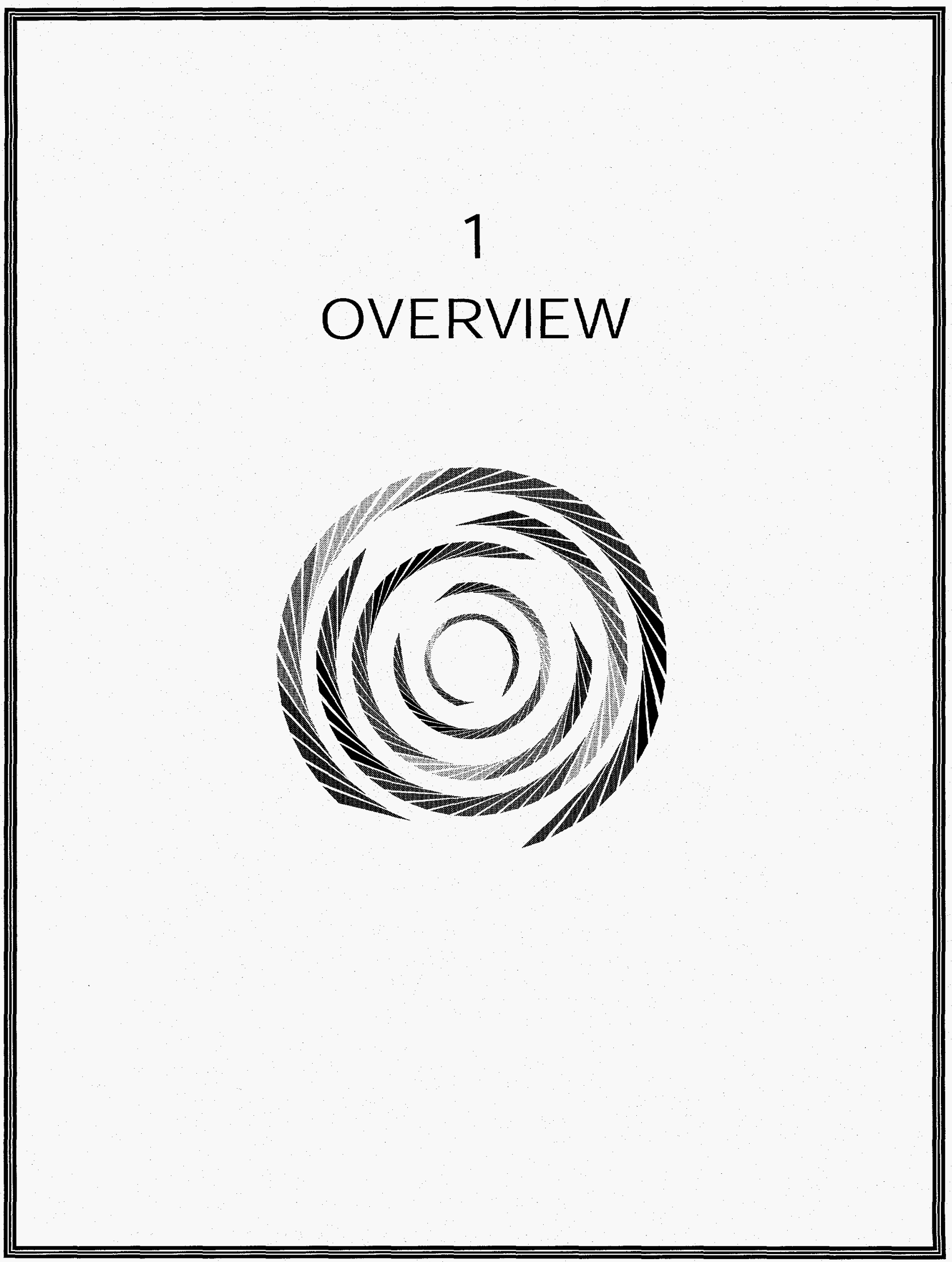




\subsection{Background}

This Progress Report summarizes the activities of the Experimental Facilities Division (XFD) in support of the users of the Advanced Photon Source (APS), primarily focusing on the past year of operations. The personnel of XFD are proud of their progress and accomplishments and hope that the reader will share their dedication and enthusiasm in supporting the APS users.

Operation of the APS is prominent among Argonne National Laboratory's scientific endeavors. In February 1996, the Office of Basic Energy Sciences of the U.S. Department of Energy (DOE) confirmed the completion of the construction of the APS Project. The dedication of the Project followed on May 1, 1996. In September 1996, the APS began operations as a national user facility serving the U.S. community of $\mathrm{X}$-ray researchers from private industry, academic institutions, and other research organizations. The start of operations was about three months ahead of the baseline date established in 1988.

The responsibility for the construction of the technical components of the APS Project was divided between the Accelerator Systems Division (ASD) and XFD. Throughout the construction period, the DOE conducted reviews, using technical consultants, of the progress of the work. When the construction phase of the Project was completed, ASD and XFD took operational responsibilities. The progress in the operational activities of the APS is now reviewed by appropriate committees appointed by The University of Chicago, which operates Argonne National Laboratory (ANL) for the DOE.
Thirty-five of the forty storage-ring sectors include both insertion-device radiation sources and bending-magnet sources. Of these 35 sectors, one sector is dedicated to ASD for particle beam diagnostics using the radiation from an undulator source and a bendingmagnet source. The remaining 34 sectors will be used for research and development (R\&D) work by the APS users.

The APS commissioning goal of extracting undulator radiation was met by XFD on August 18, 1995. This permitted the XFD to measure the particle beam emittance and the $x-y$ coupling using undulator $x$-rays. Successful commissioning of the beamline radiation sources and front ends demonstrated the capability of this equipment to operate at a level commensurate with the technical performance goals. In addition, successful "acceptance testing" and beamline safety reviews demonstrated that the initial complement of beamlines have been developed and can be operated safely while meeting technical specifications.

In the first phase of this project, 20 of these 34 sectors are being instrumented behind the storage-ring shield wall to deliver undulator or wiggler radiation, as well as bending-magnet radiation, to the APS users. During the past year, XFD personnel have installed and commissioned insertion devices and beamline front ends consistent with the APS user requirements. In addition, the Personnel Safety Systems on all user experiment stations have been designed, installed, validated, commissioned, and operated by XFD personnel to meet users' objectives. Many of the "standard and modular" beamline components required by the user community have been designed, constructed, and tested for their performance by XFD. The design drawings of all these components are available 
to the user community in electronic format on the "Design Exchange" established by XFD.

With the construction project completed, during the past year XFD has enhanced its mission to prepare fully for facility operation (which began in September of 1996) and to increase its support for APS user beamline operations. Currently, 18 of the 20 undulators and wigglers required by the APS users have been installed on the storage ring, 35 of the 40 beamline front ends have been installed, and 22 (16 insertion-device and 6 bending-magnet) user beamlines in 16 of the 20 sectors, including 35 experiment stations, have started commissioning activities. These beamlines are routinely taking radiation to both commission instruments, such as monochromators, mirrors, slits, focusing optics, diffractometers, goniometers, detectors, and beamline control electronics, and to perform early research. The XFD has worked hard to provide $\mathrm{X}$-rays very early in the facility operations phase for APS users to do innovative experiments.

\subsection{Mission of the APS Experimental Facilities Division}

The mission of the XFD is consistent with the vision of the APS to function as a reliable and preeminent source of synchrotron radiation for APS users.

XFD believes that we can best serve the APS users by investing in three important goals: reliable and successful operation, high-quality user technical and administrative support, and innovative $R \& D$ in support of user operations. These goals enable us to go beyond the traditional role of DOE user facilities to create an intelligent partnership with our users.
We commit ourselves to an organization that shares the following principles:

- understanding our users' operational goals and striving to exceed their needs

- providing seamless support to our users in all areas

- creating a rewarding, enriching, and collaborative $R \& D$ environment for our staff and the users to facilitate long-term success of the APS as the premier user facility in the world

- expanding our worldwide leadership role in the synchrotron radiation community

- assuring the safety of APS users, visitors, and APS/XFD personnel, and the protection of the environment

- approaching our daily work with enthusiasm, dedication to users and a sense of humor

\subsection{APS User Sector Layout}

In Figure 1.1, the layout of the APS experiment hall floor and the allocation of sectors to various Collaborative Access Teams (CATs) is shown. Each sector consists of two beamlines, one based on an insertion-device source and the other based on a bendingmagnet source. The insertion devices in 18 sectors are undulators, one sector has a conventional wiggler, and the last has an elliptical multipole wiggler for the production 


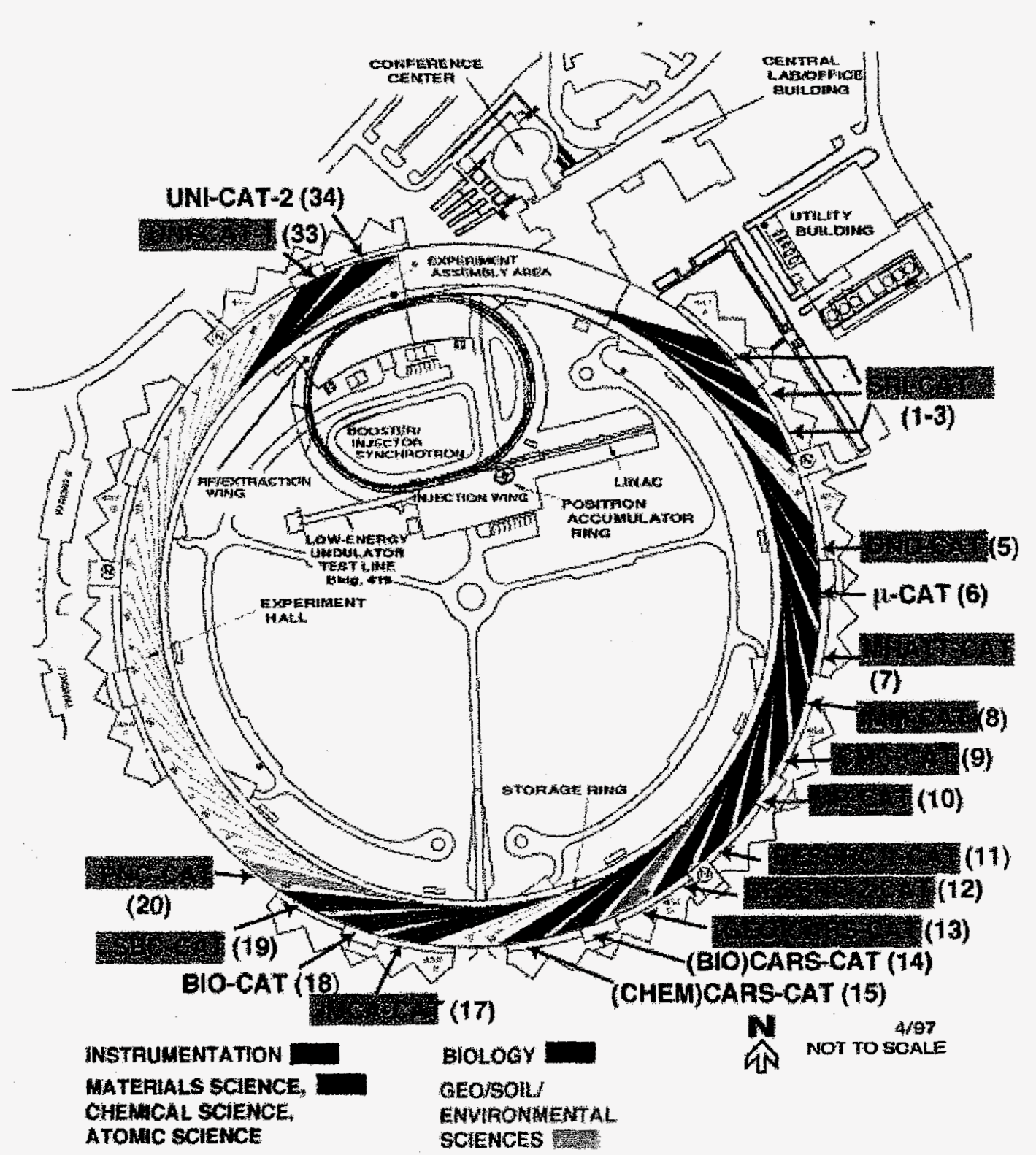

Fig. 1.1 APS Collaborative Access Teams by sector and discipline

of circularly polarized $\mathrm{x}$-rays with a capability to switch the polarization helicity. Some of the CATs have taken responsibility for more than one sector. The progress of the CAT beamline construction and scientific work is evaluated at regular intervals by the APS Program Evaluation Board (PEB). The lead institutions developing the beamlines in each of the sectors is presented in Table 1.1, which also indicates the diverse scientific disciplines and technological applications being addressed by the CATs. In Figure 1.1 , a broad classification of scientific interests has been shown. They are (a) condensed matter physics, chemical science, and material science, (b) health science, (c) environmental science, geoscience, soil science, and agricultural science, and (d) synchrotron 
Table 1.1 Lead Institutions and Research Areas of the APS Collaborative Access Teams

\begin{tabular}{|c|c|c|}
\hline Collaborative Access Team & Institution & Scientific \& Technological Applications \\
\hline $\begin{array}{l}\text { Basic Energy Sciences } \\
\text { Synchrotron Radiation Center } \\
\text { (BESSRC, Sectors } 11 \& 12 \text { ) }\end{array}$ & Argonne & $\begin{array}{l}\text { Materials science; chemistry; atomic physics; } \\
\text { geosciences. Environmental remediation. }\end{array}$ \\
\hline $\begin{array}{l}\text { Biophysics } \\
\text { (Bio, Sector 18) }\end{array}$ & $\begin{array}{l}\text { Illinois Institute of } \\
\text { Technology }\end{array}$ & $\begin{array}{l}\text { Biophysics, improved understanding of the } \\
\text { mechanism of action of enzymes. Industrial enzymes. }\end{array}$ \\
\hline $\begin{array}{l}\text { Center for Real-Time X-Ray Studies } \\
\text { (MHATT, Sector 7) }\end{array}$ & Howard University & $\begin{array}{l}\text { Physics; materials science; chemistry. Liquid } \\
\text { crystals; solid-state lasers. }\end{array}$ \\
\hline $\begin{array}{l}\text { Complex Materials } \\
\text { (CMC, Sector 9) }\end{array}$ & $\begin{array}{l}\text { Exxon Research and } \\
\text { Engineering }\end{array}$ & $\begin{array}{l}\text { Chemistry of complex materials. Polymers, liquid } \\
\text { crystals, and membranes. }\end{array}$ \\
\hline $\begin{array}{l}\text { Consortium for Advanced Radiation } \\
\text { Sources } \\
\text { (CARS, Sectors } 13,14, \& 15 \text { ) }\end{array}$ & $\begin{array}{l}\text { The University of } \\
\text { Chicago }\end{array}$ & $\begin{array}{l}\text { Structural biology; geoscience; chemical, material, } \\
\text { and soil/environmental sciences; improved } \\
\text { understanding of earth and of biological processes at } \\
\text { the molecular level. Environmental remediation; } \\
\text { materials and catalysts; plant fertilizers. }\end{array}$ \\
\hline $\begin{array}{l}\text { E.I. DuPont de Nemours and Co.- } \\
\text { Northwestern University-Dow } \\
\text { Chemical Co. } \\
\text { (DND, Sector 5) }\end{array}$ & $\begin{array}{l}\text { Northwestern } \\
\text { University }\end{array}$ & $\begin{array}{l}\text { Materials science; polymer chemistry. Technologies } \\
\text { for microfabrication and electrodeposition; corrosion } \\
\text { prevention: polymer fibers. }\end{array}$ \\
\hline $\begin{array}{l}\text { IBM-MIT-McGill } \\
\text { (IMM, Sector } 8 \text { ) }\end{array}$ & $\begin{array}{l}\text { Massachusetts } \\
\text { Institute of } \\
\text { Technology }\end{array}$ & $\begin{array}{l}\text { Materials science and physics. Products for high } \\
\text { technology sector. }\end{array}$ \\
\hline $\begin{array}{l}\text { Industrial Macromolecular } \\
\text { Crystallography Association } \\
\text { (IMCA, Sector } 17 \text { ) }\end{array}$ & Procter and Gamble & Structural biology. Designer drugs. \\
\hline $\begin{array}{l}\text { Materials Research } \\
(\mathrm{MR}, \text { Sector } 10)\end{array}$ & $\begin{array}{l}\text { University of } \\
\text { Notre Dame }\end{array}$ & $\begin{array}{l}\text { Materials science. Polymers (e.g., for textiles); } \\
\text { environmental remediation; complex chemicals. }\end{array}$ \\
\hline $\begin{array}{l}\text { Midwest Universities } \\
(\mu, \text { Sector } 6)\end{array}$ & Iowa State University & $\begin{array}{l}\text { Materials science. Synthetic membranes for } \\
\text { biological processes. }\end{array}$ \\
\hline $\begin{array}{l}\text { Pacific Northwest Consortium } \\
\text { (PNC, Sector 20) }\end{array}$ & $\begin{array}{l}\text { University of } \\
\text { Washington }\end{array}$ & $\begin{array}{l}\text { Environmental and materials science. Environmental } \\
\text { cleanup; moldable ceramics. }\end{array}$ \\
\hline $\begin{array}{l}\text { Structural Biology Center } \\
\text { (SBC, Sector 19) }\end{array}$ & Argonne & Structural biology. Enzymes and new drugs. \\
\hline $\begin{array}{l}\text { Synchrotron Radiation } \\
\text { Instrumentation } \\
\text { (SRI, Sectors } 1,2, \& 3 \text { ) }\end{array}$ & Argonne & $\begin{array}{l}\text { Synchrotron radiation instrumentation and } \mathrm{x} \text {-ray } \\
\text { physics. Insertion devices and optical elements for } \\
\text { the APS users and other synchrotron } x \text {-ray facilities. }\end{array}$ \\
\hline $\begin{array}{l}\text { University-National } \\
\text { Laboratory-Industry } \\
\text { (UNI, Sectors } 33 \text { \& 34) }\end{array}$ & University of Illinois & $\begin{array}{l}\text { Materials science; structural biology. Molecular } \\
\text { sieves for catalysts. }\end{array}$ \\
\hline
\end{tabular}


radiation instrumentation. It is in this last area that XFD has a major commitment. Through the Synchrotron Radiation Instrumentation (SRI)-CAT, we support the needs of all the CATs.

\subsection{XFD Organization}

The XFD was recently reorganized to meet the mission of the Division. The three functional areas of XFD are:
A. User Operations
B. User Administrative and Technical Support

C. R\&D in Support of User Operations

The major objectives of the functional areas are given in Fig. 1.2 and are described in more detail in other parts of this document.

The XFD organization structure, shown in Fig. 1.3, defines various groups by specialization. This structure guarantees excellent communication and interaction across the boundaries of the groups to meet both the group and XFD objectives. The groups consist of a Group Leader and Principal Investigators with well-defined technical and supervisory responsibilities.

\subsection{APS User Operations}

User Operations is supported by the Beamline Operations Group, the Safety Interlocks and Instrumentation Group, and the Floor Coordinators. Their main objective is to assure the highest level of reliability of operations of the radiation sources, beamline components, and Personnel Safety Systems to support the highest level of productivity from the APS users.

These groups are responsible for the installation, commissioning, maintenance, improvement, and operations of insertion devices and beamline front ends. In addition, the groups also perform installation, commissioning, maintenance, improvement, and operations of the personnel safety and interlock systems.

These groups also gather data to perform operations reliability studies and analysis. The staff performs analysis to evaluate failure trends and assess reliability. In addition, the Floor Coordinators support beamline commissioning and oversight of operational safety.

\subsection{APS User Administrative and Technical Support}

This activity is supported by many groups: User Administration and Support, User Technical Interface, X-ray Optics Metrology and Fabrication, Beamline Controls and Data Acquisition, and staff involved in the operations of the Design Exchange. These groups provide seamless support to the APS users in all areas. They specifically provide

- Technical support on the experiment hall floor

- Technical support on instrumentation

- Administrative support including user safety and training 


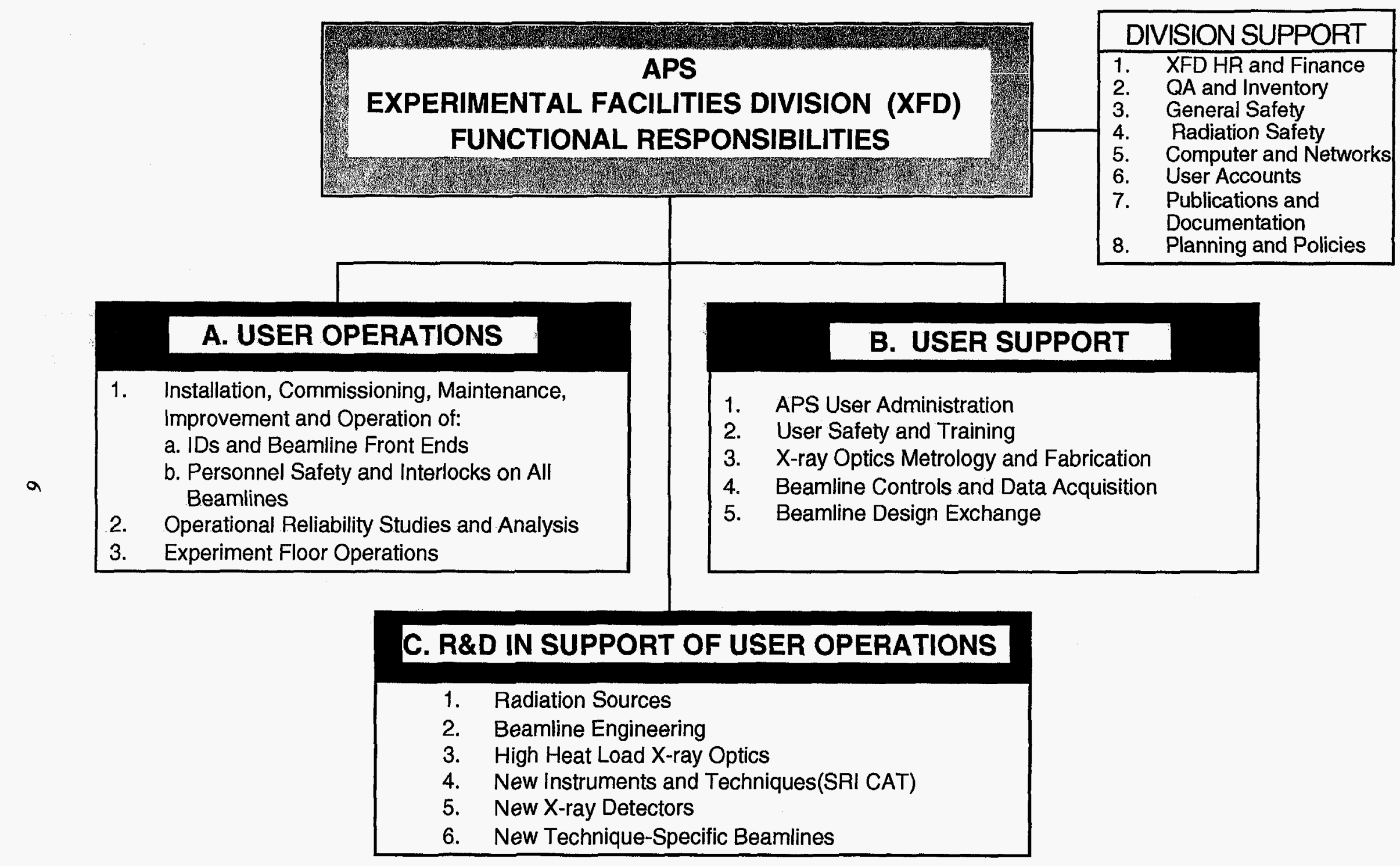

Fig. 1.2 Functional organization of the Experimental Facilities Division 


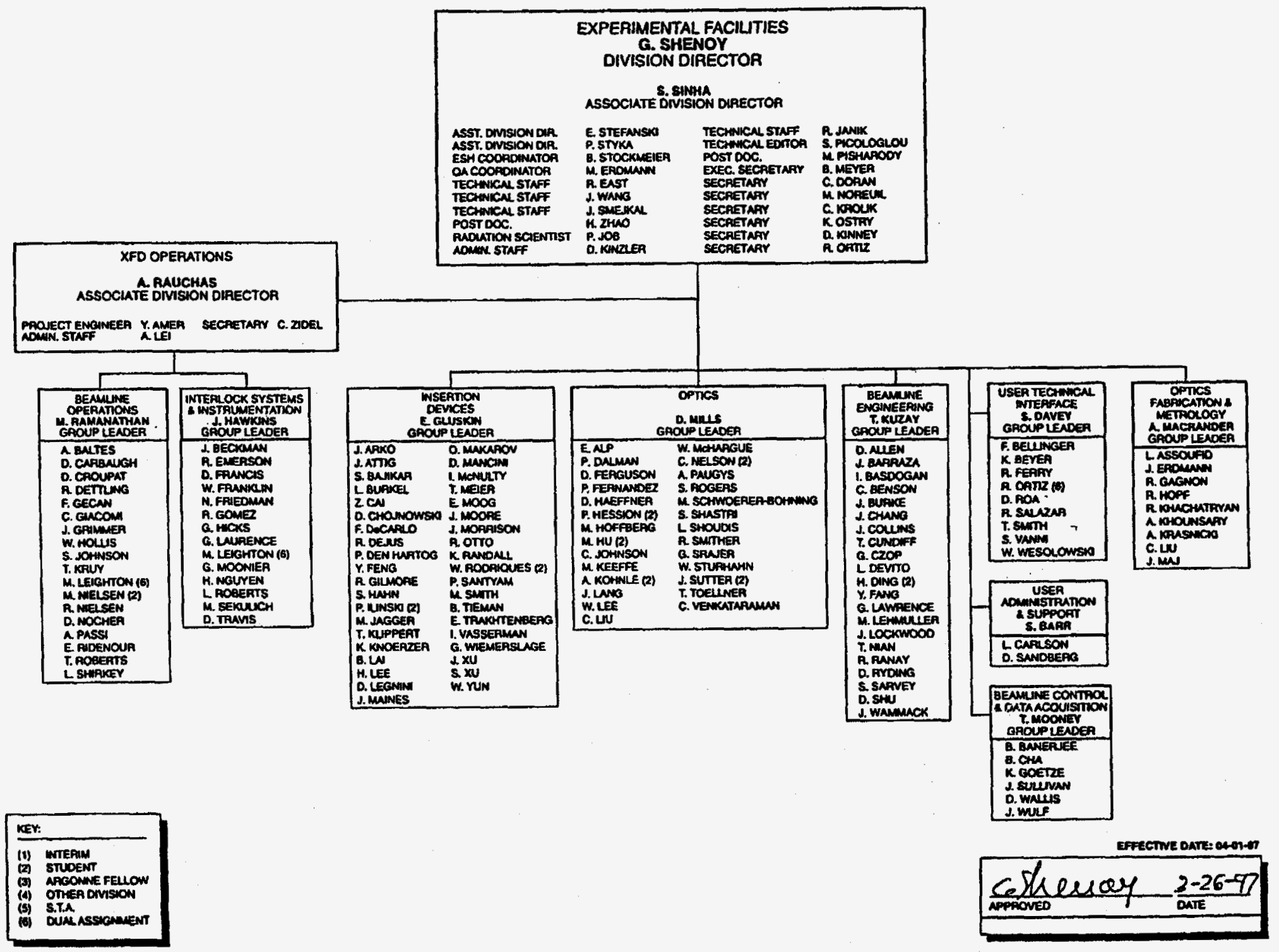

Fig. 1.3 Experimental Facilities Division organization chart 
- Development of user policies and procedures

- Development and maintenance of electronic databases to manage administrative data on APS users, and to manage all APS beamline designs required by the APS users and accessed through the World Wide Web (or Web) on the Internet.

\subsection{R\&D in Support of User Operations}

$R \& D$ in support of user operations is primarily supported by the Insertion Device Group, Beamline Engineering Group, and $\mathrm{X}$-ray Optics Group. These groups support the Operations Groups by carrying out any major maintenance, repairs and upgrades required to successfully operate the components behind the shield wall. These groups also perform $R \& D$ to address future APS operational parameters, such as the "topoff' mode, $300 \mathrm{~mA}$ operation, and unique radiation sources. They also provide the highest quality leadership in synchrotron instrumentation and technique development in order to support and enhance the APS users' scientific goals. Some of these activities with both short- and long-term benefits to the user community are performed through SRI-CAT.

The experience of the personnel in these groups is extensive. Their work at the APS has produced numerous publications and patents. (See Appendix 1 for recent publications and Appendix 2 for patents.)

\subsection{SRI-CAT}

The SRI-CAT has the principal mission of developing new and unique forefront instruments and techniques to advance the use of synchrotron radiation. The principal developers of the SRI-CAT are members of XFD (from the Insertion Device Group, Beamline Engineering Group, and X-ray Optics Group), and their primary responsibility is to perform R\&D in support of user operations. Recently, new members have joined SRI-CAT from Purdue University, University of Houston, National Institute of Standards and Technology, Lawrence Berkeley National Laboratory, Stanford Synchrotron Radiation Laboratory, Cornell High Energy Synchrotron Source (CHESS), and many Australian institutions managed by the Australian Nuclear Science and Technology Organization.

Consistent with the main focus of SRI-CAT to support the needs of all the CATs, the following is a list of major accomplishments:

- Design, construction, and operation of standard and specialized insertion devices

- Diagnostics of radiation from the insertion devices

- Development of high heat load optics

- Design, construction, and testing of various "standard and modular" beamline components 
- Evaluation of the performance of the EPICS-based beamline control and data acquisition hardware and software that will be used by many other CATs

- Initial evaluation and scrutiny of all beamline commissioning and operational procedures developed for the user beamlines by XFD Operations

- Evaluation of logic and technical performance of Personnel Safety Systems implemented on various experiment stations of SRI-CAT.

- Evaluation of the shielding performance of various XFD-designed experiment stations

- Testing of all safety procedures developed to support the APS user community

Towards the development of unique synchrotron techniques that are not generally available at other APS CAT beamlines, some members of SRI-CAT have defined objectives for a set of "strategic" instruments. These instruments are in an advanced stage of commissioning, and some are already being used by members of SRI-CAT to perform planned research. A short list of accomplishments to date is given below:

- Application of microbeam techniques

- Application of coherence-based techniques

- Development of 0.5 to $4 \mathrm{keV}$ scientific capabilities
- Development of ultrahigh-resolution inelastic scattering techniques

- Application of nuclear coherent spectroscopy

The above accomplishments and future plans of SRI-CAT are discussed in more detail elsewhere in this document.

\subsection{Collaborative Work with APS Users and Other Synchrotron Radiation Facilities}

In 1991, XFD established a Collaborative Research Program (CRP) with potential users of the APS. In this program, a joint proposal is made by XFD staff members and interested APS users to perform R\&D on a topic of general interest to APS users and to which both collaborating parties can bring their expertise and institutional resources. This program has led to many interesting results. During the past few years, owing to the heavy work load associated with beamline construction, the users have not responded to calls for CRP proposals. This program will begin to interest the users again as they finish commissioning of their beamlines. The APS users have also developed joint $R \& D$ proposals with XFD staff on problems of common interest to seek funds from new sources.

In recent years, the XFD staff has supported major activities related to construction at various other synchrotron facilities including NSLS, BESSY II, HASYLAB, and SPring-8, reflecting the regard of the worldwide synchrotron radiation community for the work performed by XFD staff. 


\subsection{Long-Term Strategic Plan for XFD}

Strategic planning has been performed by XFD to develop new initiatives to meet future APS goals (described in Chapter 6). Considerable $R \& D$ is required in order to prepare the initiatives. Support for such $R \& D$ activities is currently not provided through the funding obtained from DOE. Hence support is derived mainly from the Laboratory Directed Research and Development (LDRD) funds distributed by the Argonne National Laboratory Director through a laboratory-wide competitive process. The XFD LDRD programs funded for the period 1996-1998 include:

1. CVD Diamond Imaging Detector (97-98)

2. Deep Etch Lithography (96-98)

3. Intensity Fluctuation Spectroscopy (9698)
4. Heterodyne Correlation Spectroscopy (9698)

5. Fabry-Perot Cavity for Sub-meV Resolution (97-99)

6. Anomalous Inelastic Scattering with $\mathrm{meV}$ Resolution (97-99)

7. Computational Analysis to Specify the Requirements of an Undulator to Demonstrate the SASE Concept and Prototype Testing (97-99)

XFD also constantly seeks other sources of funds to support $R \& D$ ideas that can either lead to new capabilities for the users or support possible new initiatives. Some of these are discussed in this Progress Report. 

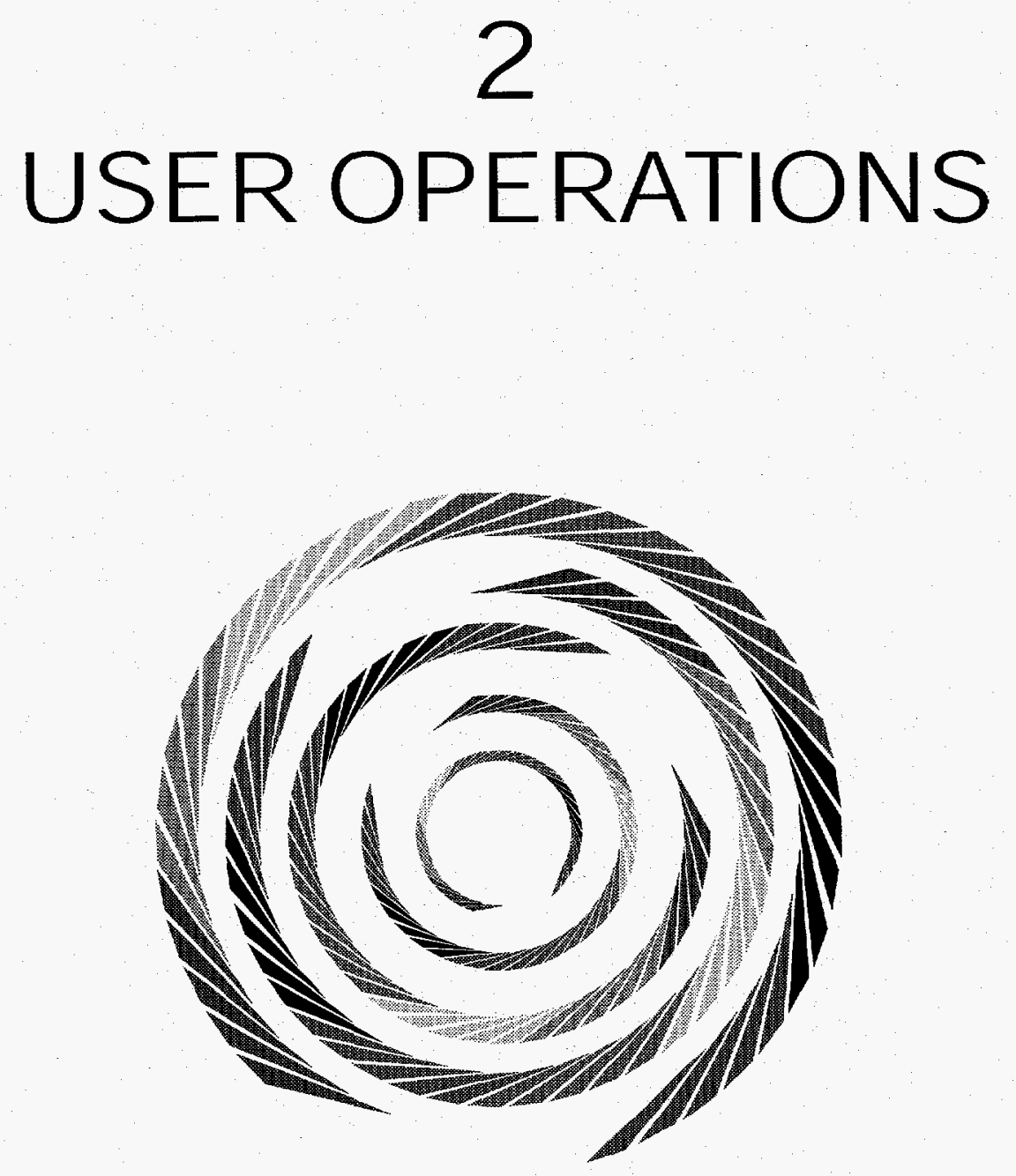


\subsection{Introduction}

\subsubsection{Advanced Photon Source Facility Description}

The APS was designed and constructed to be a major national user facility providing high-brilliance $\mathrm{x}$-ray beams for users from academic institutions, national laboratories, governmental bodies, and industry. The APS uses recently developed insertion-device technology and low-emittance particle beam to produce high-brilliance beams of $\mathrm{x}$-rays.

Figure 2.1 shows a plan view of the APS. The main feature of the APS facility is the experiment hall building, an annular structure with an exterior radius of 191.4 meters, an inner radius of 164.6 meters, and a height of 9.8 meters. The building houses the experiment hall, where $\mathrm{x}$-ray beamlines and experimental equipment are located, and a storage ring contained within a concrete shielding enclosure. Around the periphery of the experiment hall building are laboratory and office modules (LOMs), which provide office and laboratory space for CAT members and independent investigators performing experiments at the APS. A central laboratory and office building (CLO) is located at the northern periphery of the experiment hall building. The CLO houses the facility staff, who are responsible for operating and maintaining the facility and supporting the experimental program. Adjoining the CLO is the multifunction wing, providing facilities for meetings and conferences. Separating the CLO from the experiment hall building is the control center, which provides a central location for controlling the operation of the facility. To the southeast of the CLO is the utility building, which provides the electrical power, deionized water, and other utilities for the APS facility.

The storage ring is contained within a concrete shielding enclosure located at the inner radius of the experiment hall building. The storage ring consists of magnets, vacuum systems, and other equipment necessary to maintain the circular orbit and energy level of a beam of positrons that is circulating within the storage ring. As the positrons are bent around the circular orbit, they release energy in the form of $\mathrm{x}$-rays. The $\mathrm{x}$-ray production can be greatly enhanced by the inclusion of magnetic insertion devices (IDs) in the storage ring. Two types of IDs are currently used: wigglers, which produce very intense $\mathrm{x}$-ray radiation over a wide range of energies, and undulators, which yield pseudomonochromatic $\mathrm{x}$-ray radiation tunable over a wide energy range at high brilliance. The $\mathrm{x}$-rays are transmitted from the storage ring through beamline front ends (FEs), which provide a means for confining, defining, and/or stopping each $\mathrm{x}$-ray beam before it exits the storage-ring tunnel to the beamline. The beamlines are located in the experiment hall, between the outside of the concrete shielding enclosure and the outer wall of the experiment hall building. The beamlines include shielded enclosures, beam transports containing vacuum systems, optical elements and experimental instrumentation, and $\mathrm{x}$-ray detectors.

\subsubsection{APS Operations}

The Associate Laboratory Director for the APS is ultimately responsible for the successful operation of the APS. The day-today responsibilities for operations are entrusted to two major divisions of the APS, 


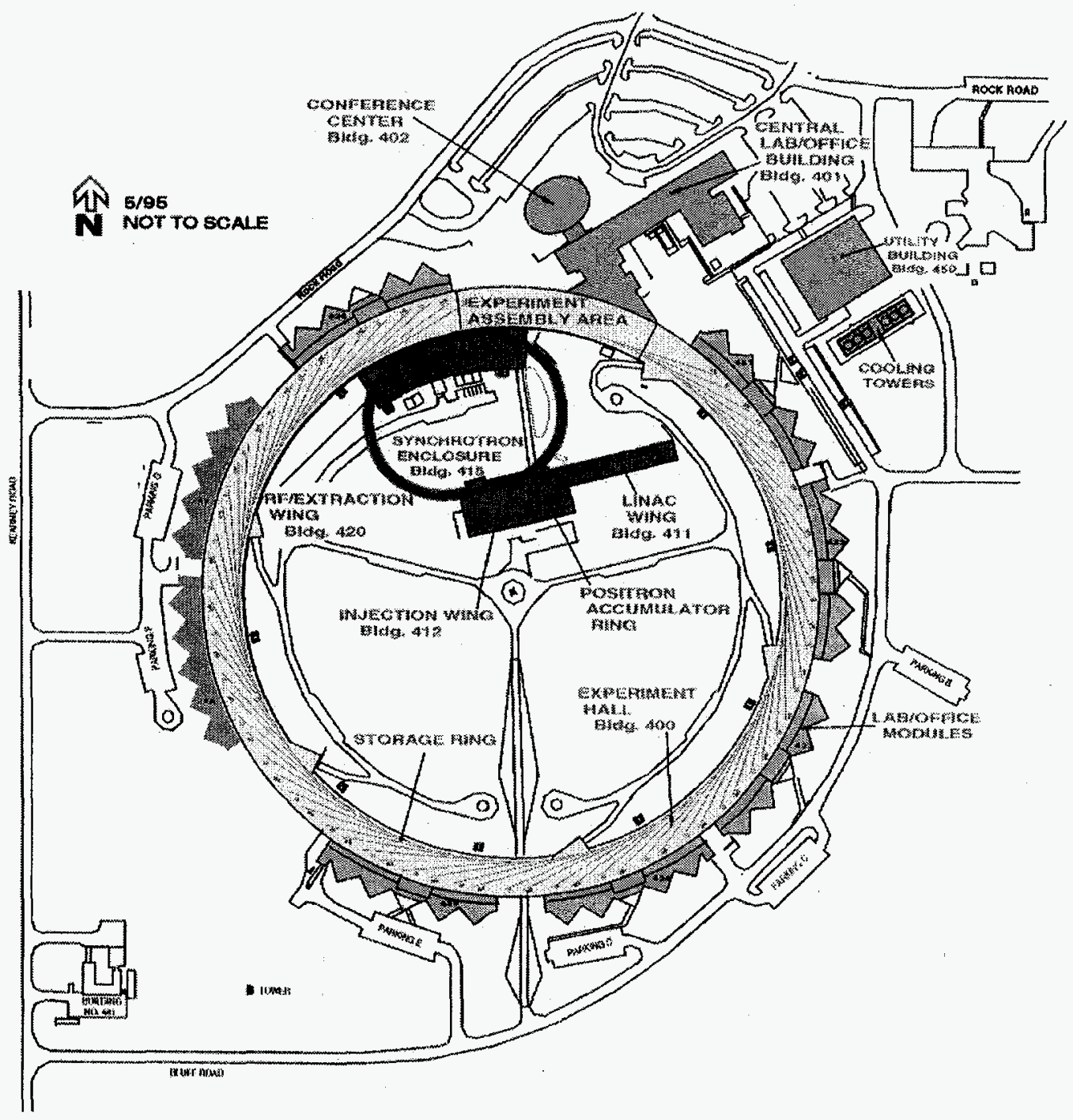

Fig. 2.1 Plan view of the Advanced Photon Source

ASD and XFD. The Accelerator Systems Division has constructed and commissioned the APS accelerators and has responsibility to maintain, operate, and improve the accelerators so as to achieve the highest possible availability and reliability of beam for the user community. The Experimental Facilities Division has constructed and commissioned all IDs, beamline FEs, and all beamline safety systems, and has the responsibility to maintain, operate, and improve the performance of these systems to fully support the users' goals. The principal staff from the Associate Laboratory Director's office, as well as from ASD and XFD, form the Operations Directorate, which meets every week to make all major operations decisions and to determine the operational schedules.

\subsubsection{XFD Operations Organization}

The XFD Operations Organization is responsible for assuring that the APS effectively 
meets the operational needs of the user community and for assuring that XFD and user activities conform to the applicable requirements of the Accelerator Safety Order, DOE 5480.25.1 The XFD Operations Organization has participated with ASD in generating a Final Safety Assessment Document (SAD), ${ }^{2}$ as required by the DOE order, which provides an evaluation of the level of risk, level of consequence, and the probability of occurrence for the various hazards found at the facility. The SAD also defines the operating safety envelope for the facility. All of the operational activities conform to the requirements as stated in the APS Conduct of Operations Manual. ${ }^{3}$ In support of APS user activities, the XFD Operations Organization also gathers specific facility operating requirements, integrates the requests, determines the operating modes that are needed to meet the requirements, and works with the ASD to satisfy the requirements. The Operations Organization is under the direction of an Associate Division Director for Operations and includes two major groups: the Beamline Operations Group and the Interlock Systems and Instrumentation Group, as well as support staff to aid the group activities. The XFD Operations organization is shown in Fig. 1.3 (in Chapter 1).

The Beamline Operations Group is charged with the responsibility for installing, operating, and maintaining the IDs, ID

1 Safety of Accelerator Facilities, DOE Order 5480.25, U.S. Department of Energy, Washington, $D C, 1992$.

2 Advanced Photon Source Final Safety Assessment Document, APS-3.1.2.1.0, Argonne National Laboratory, Argonne, IL, June 1996.

3 Advanced Photon Source Conduct of Operations Manual, APS-3.1.1.1.0 (Revision 00, Argonne National Laboratory, Argonne, IL, June 1993. vacuum chambers, the beamline FE components, and ancillary systems. The group members have the vacuum, mechanical, survey and alignment, and utility distribution expertise that is needed to keep these components operating reliably with minimal impact on the availability of beam time to the users.

The Interlock Systems and Instrumentation Group is charged with the responsibility for generating and/or supporting the design, installation, testing, and maintenance of the Personnel Safety System (PSS), Equipment Protection System (EPS), and FE instrumentation. This includes any and all documentation, testing, and fieldwork required for supplying the XFD with high-reliability systems. Each system consists of numerous subsystems that are highly reliable and failsafe. The PSS is a redundant interlocked system that monitors personnel access into beamline enclosures. The EPS is an interlocked system that reduces the risk of damage to $\mathrm{FE}$ beam transport equipment. The group is organized into three functional blocks. The Interlock System Design Section provides interlock systems requirements, scheduling, budget/cost development and control, drafting, and project management support. These systems are designed to applicable codes, orders, and standards for such systems. Software is developed in the Software Development Section under the Software Development Plan and conforms to the Laboratory's Software Quality Assurance Plan. The hardware function in the Hardware Design Section relates to the design, systems requirements, scheduling, budget/cost, drafting, and project management support of $\mathrm{FE}$ instrumentation.

The support staff consists of engineering support personnel for quality assurance and reliability analysis, as well as a radiation scientist to provide guidance and support to 
the facility and users, especially with regard to user shielding needs.

\subsection{Beamline Technical Description}

\subsubsection{Introduction}

Generally, an experimental beamline consists of four functional sections. The first section is the source of the $x$-rays, where $x$-rays are produced from the circulating positron beam inside the storage ring. The APS provides the $\mathrm{X}$-ray radiation from two types of sources: one is IDs, and the other is bending magnets (BMs), which are dipole magnets of the storage ring. The IDs, located in the storagering straight sections, can be further tailored to provide radiation with specific characteristics required by each beamline user. The radiation from the BM source, whose primary function is to maintain the positron beam in a closed orbit, is a white-radiation source with a critical energy of $19.5 \mathrm{keV}$.

The second section, immediately outside the technical components of the storage ring but still inside the concrete storage-ring shielding tunnel, is the beamline FE section. This section contains safety shutters, photon beam stops, and other components to coarsely define the emerging $x$-ray beam and, if required, to stop the $\mathrm{x}$-ray beam and provide adequate radiation protection to areas outside the concrete shielding tunnel. Each x-ray source requires an $\mathrm{FE}$, although there are minor differences between the FEs used with an ID and those used with a BM source.

The third and fourth sections of the beamline are located on the experiment hall floor, outside the storage-ring tunnel. The third section contains hard x-ray optical elements, such as crystal monochromators, filters, and/or mirrors, which are designed to handle the power loads and tailor the characteristics of the photon beam to satisfy the user requirements. Generally, these are contained in the first optics enclosure (FOE). The fourth section consists of the experiment stations, which contain experimental instruments; the sample under investigation; any additional optics needed to analyze and characterize the scattering, absorption, or imaging process; and the detectors. The beam transports, which define and shield the beam path between the various experiment stations, are included as required. Some of the photon beams are split, so that each beamline can have multiple branch lines and numerous experiment stations.

An APS sector includes a beamline based on an ID and an adjacent BM-based beamline. The APS provides the $\mathrm{x}$-ray sources and FEs (sections one and two) for each sector. Sections three and four are the responsibility of each CAT, which designs, builds, and operates the beamlines in its sector(s) with funds independently obtained from various funding sources. Each beamline constructed by the CATs will be unique and will support the scientific program planned by the CAT. Similar components may be used in more than one beamline, but these components will frequently have different mechanical, operational, or scientific characteristics appropriate for the research being conducted on that particular beamline.

The x-ray beam produced by an ID or a storage-ring $\mathrm{BM}$ and transported through the beamline $F E$ requires some modification before it can be used as an experimental tool. The beam is tailored by optical elements, located at various positions in the beamline to support the CAT's scientific goals. Because an interaction of the full-energy $x$-ray beam with any material, even air, will result in 
scattered radiation, the optical elements, as well as all experimental instruments, are generally located within shielded enclosures to prevent access by personnel when the x-ray beam is on. These are referred to as experiment stations. To ensure that radiation levels on the experiment hall floor meet required safety standards, the vacuum beam pipe, through which the x-ray beam is transported, is shielded.

\subsubsection{Operational Aspects of IDs}

The characteristics of various IDs installed on the APS storage ring are discussed in detail in Chapter 4 (section 4.1). When the construction phase of the project has been completed, 20 IDs will have been installed for providing $\mathrm{x}$-ray beams to the users. Of these, 18 are undulators (most with a magnetic period of $3.3 \mathrm{~cm}$ ), one is a wiggler with a critical energy of $33 \mathrm{keV}$, and one is an elliptical multipole wiggler (EMW) to provide circularly polarized radiation in the plane of the positron orbit.

A typical ID is C-shaped, open on the side near the ID vacuum chamber, and closed on the side near the storage-ring aisle. An end view of a typical ID is shown in Fig. 2.2. The ID base is separate from the supports of the vacuum chamber, allowing an ID to be removed or installed without affecting the vacuum chamber and the ultrahigh-vacuum (UHV) atmosphere within it. The vacuum chamber can thus be baked out, the vacuum level certified, and the chamber precisely aligned to the beam center line prior to ID installation and alignment.

The ID is supported from its internal frame on three points by screw jacks. These are used to level the ID and to uniformly center the gap

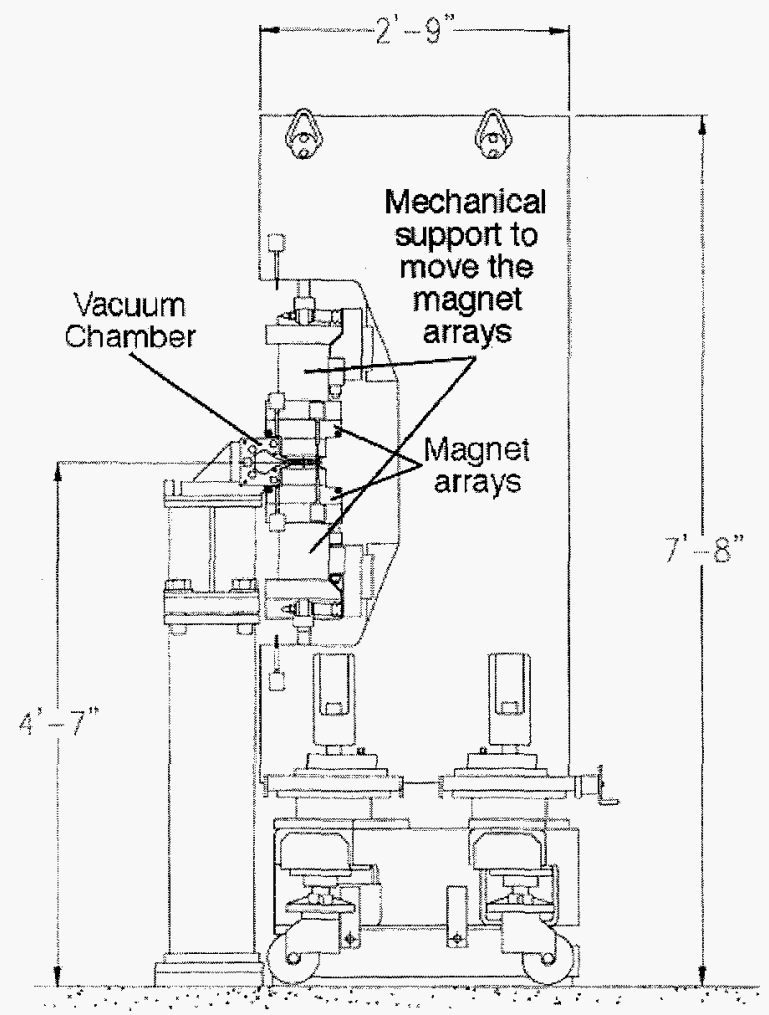

Fig. 2.2 End view of a typical ID

above and below the vacuum chamber. The screw jacks are in turn mounted to dovetail slides, which are used to adjust the horizontal position of the ID relative to the vacuum chamber.

The permanent magnets used in IDs are extremely strong. The support system of each APS ID maintains very precise alignment (better than 10 microns over the length of the undulators) between the upper and lower magnet arrays (each of which is about $2.5 \mathrm{~m}$ long) and the ID vacuum chamber. The drive system must overcome the attractive force between the upper and lower magnet arrays; in some wigglers this force may approach 15,000 lbs. The drive system must also position the magnet arrays such that the gap between them can be repetitively controlled with an error of less than 10 microns. The magnet arrays are routinely closed to gaps of 
$11 \mathrm{~mm}$ with a chamber slightly thicker than $10 \mathrm{~mm}$ between them. The need to position the magnet arrays as close to the beam as possible and to avoid breaching the vacuum chamber necessitates mechanically fail-safe drive systems. The IDs utilize two independent drive systems, one on the upstream end and one on the downstream end of the device. This allows the gap between the magnet arrays to be tapered. Several levels of safety are also provided by the control system to provide broader energy bandwidths for various undulator harmonics required for specific experiments.

The magnet arrays of the ID sandwich a specially designed storage-ring vacuum chamber (Fig. 2.3). The outside height of the portion of the vacuum chamber located within the magnet arrays is as small as possible to allow the separation between the magnet arrays to be minimized for the largest possible magnet field. The wall thickness of the vacuum chamber has also been minimized. Details are provided in Chapter 4 (section 4.1).

\subsubsection{The Beamline FE Operation}

The APS beamline FEs are designed to be standard, with one type (Fig. 2.4) for all planned IDs (undulators or wigglers) and another type for all BMs. Depending on the user needs, the ID FEs can be of windowless configuration (with the use of differential vacuum pumps) or of conventional configuration with windows. Normal incidence heat fluxes in the ID FEs at the photon shutter locations are very high, on the order of

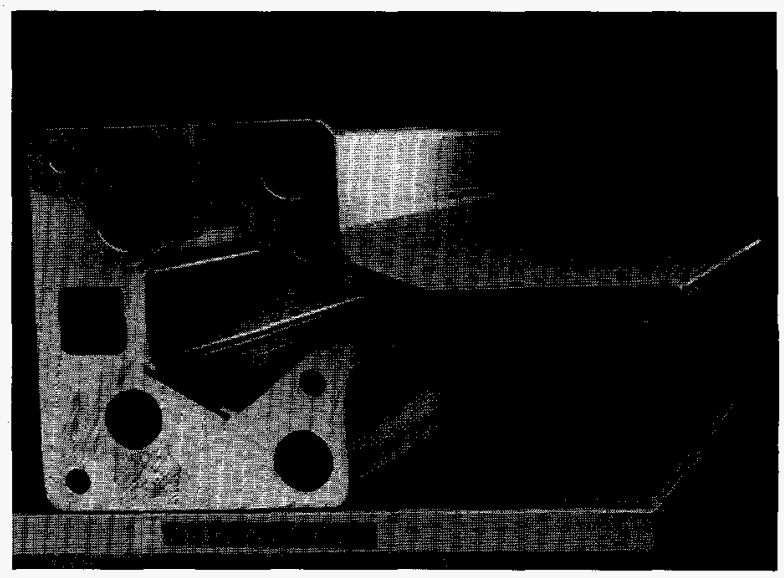

Fig. 2.3 Undulator vacuum chamber cross section

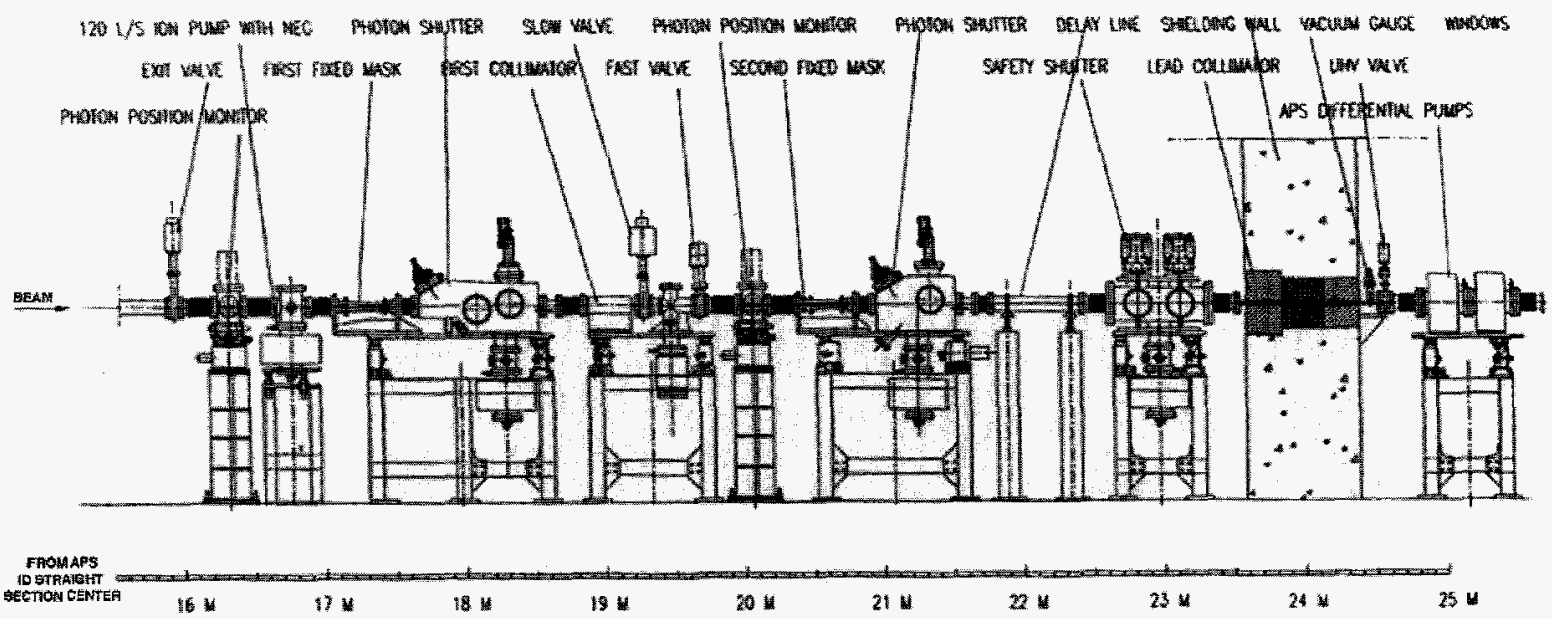

Fig. 2.4 ID front end 
$450 \mathrm{~W} / \mathrm{mm}^{2}$ or higher. Designing for these fluxes and total heat loads of $10-15 \mathrm{~kW}$ posed significant engineering and material challenges. The details of the FE designs have been published.

\section{FE Component Descriptions}

In the following, we provide a brief description of the FE components.

Storage Ring Exit Valve: This is a UHV allmetal gate valve that isolates the $\mathrm{FE}$ vacuum from the storage-ring vacuum. This valve must be open whenever beam is stored in the storage ring.

X-ray Beam Position Monitor: At the APS, each beamline FE has two sets of $\mathrm{X}$-ray beam position monitors (XBPMs) to monitor the $\mathrm{X}$-ray beam position and angle. The XBPMs measure photoelectrons generated by the sensory blades and deduce the beam position by comparison of the relative signals from the blades. Compared to the particle beam position monitors in the storage ring, the FE XBPMs have much higher positional sensitivity to the $\mathrm{X}$-ray beam and are more sensitive to angular motion because they are located far away from the source.

For the BM, the vertical position of the beam is critical. The horizontal beam position is not measured, owing to a typical FE acceptance of 6 milliradian of horizontal span of beam. The BM XBPMs consist of two molybdenum blades. The separation of the blades is precisely measured prior to installation. In the case of an undulator beamline, the beam size is small in both the $\mathrm{x}$ and $\mathrm{y}$ directions, and hence both the horizontal and vertical positions can be measured. Because of the high power from the undulator or wiggler source, chemical vapor deposition (CVD) diamond blades coated with gold are used as blades in the XBPMs (four blades each for undulator FEs and six blades each for wiggler FEs). All the blades in the XBPM are mounted on an oxygen-free high-conductivity (OFHC) copper block using ceramic insulators and are located in UHV. The copper block is water cooled. There are always two sets of XBPMs in each FE to measure both the position and the angle of the beam.

During normal operation of the APS, typical XBPM signals are of the order of microamps. The current signals from the XBPM are brought to a $4 / 8$ channel current amplifier located on top of the storage-ring tunnel. The voltage signals $(0-10 \mathrm{~V})$ are in turn fed to display units. The signal is also delivered to the APS control systems via the RS485 interface protocol.

The FE XBPMs are constantly being improved as more operational experience is gained. Some of these details are provided in Chapter 4 (section 4.2).

Fixed-Mask Assembly: This is the first FE component to interact with the beam. The fixed mask may be exposed to the full beam or part of the beam, and therefore cooling and material options must be carefully considered in its design. Each ID FE has two identical fixed-mask assemblies. Water channels are machined into the aperture walls and filled with a porous copper matrix for higher heat transfer to provide cooling.

The design of the fixed masks for BM FEs is similar to that for the ID FEs. Three fixed masks of similar construction are used in each BM FE. 
Photon Shutters: This component intercepts the total photon beam to isolate downstream components from the source. The closing time for this device is $0.5 \mathrm{sec}$. Two of these devices are installed in each FE. In the case of the ID FE, both devices include a "hockey stick" shaped copper absorber blade. The first photon-shutter blade intercepts the beam at a $1.5^{\circ}$ grazing angle, and the second photon shutter blade intercepts the beam at a $2^{\circ}$ grazing angle. The position of these devices (open or closed) is redundantly indicated by separate switches for each position. The first photon shutter is interlocked to close before the safety shutter closes to prevent a direct beam hit on the safety shutter. It is also interlocked to open only after the safety shutter is opened.

The BM photon shutters are smaller and simpler than those in an ID FE. Preliminary tests have indicated that these shutters are capable of handling the $\mathrm{BM}$ radiation from $300 \mathrm{~mA}$ of stored beam.

Collimators: The collimators in the FEs define a line of sight to the source and allow a cone of beam to pass through. This device consists of a rectangular vacuum chamber with conflat flanges on each end surrounded by lead blocks to absorb scattered x-rays and bremsstrahlung.

Slow Valve: The slow valve (SV) has the usual function in a synchrotron $\mathrm{FE}$ and is an all-metal UHV gate valve that seals to isolate the storage-ring vacuum system from a vacuum breach in the downstream FE or user's beamline. The closing time is approximately $2 \mathrm{sec}$. The slow valve is protected by first closing a photon shutter if a slow leak is sensed or by dumping the stored beam in the case of a serious vacuum breach.
Fast Valve: The fast valve (FV) is positioned immediately downstream of the slow valve. This valve closes in approximately $5 \mathrm{msec}$ but does not provide a UHV seal. It provides a low-conductance path that stops a shock wave in the event of a serious vacuum breach downstream. Triggering this device will also trigger the slow valve to isolate the upstream $\mathrm{FE}$ and storage-ring vacuum system from the vacuum breach.

Vacuum Delay Line: Because the BM beamline diameter is large, to provide some vacuum delay capability within the BM FEs, eight baffles with apertures are installed inside a 2-meter section of the transport pipe downstream of the fast valve. Such a delay line is not required in the ID FE because of the inherent constrictions in the path.

Safety Shutters: Each FE contains two safety shutters that operate simultaneously; they are used to absorb bremsstrahlung radiation from the scattered particle beam. The absorbing material is UHV-grade tungsten. In the open position, the safety shutters serve a second function as a collimator.

The BM safety shutter is of the same design as the ID safety shutter but with a larger collimator opening.

Front-End Exit Valve: The FE exit valve (FEV) is located immediately downstream of the collimator within the storage-ring shield wall penetration. The FEV is used to isolate the FE vacuum from that in the user beamline. It is pneumatically operated and is controlled by the FE EPS.

Filters: In wiggler beamlines, FE filters are used to give thermal and structural protection to the Be window. 
Window: The window is a vacuum separator located in the FOE just after the FEV; it separates the beamline vacuum from the FE vacuum.

Wiggler beamlines are equipped with double Be windows after the FEV in the FOE. During early commissioning, undulator beamlines are equipped with a special device in the corresponding location. This device (referred to as a commissioning window) consists of a pyrolytic graphite foil followed by a watercooled limiting-aperture mask. The vacuum is terminated by two Be windows.

Bending-magnet beamlines, like wiggler beamlines, are equipped with double $\mathrm{Be}$ windows after the FEV in the FOE.

Differential Vacuum Pump: When a user needs unattenuated radiation, a window cannot be used. In such cases, a differential vacuum pump is installed. Using apertures, pressure differentials of greater than two orders of magnitude have been obtained. A delay in excess of $100 \mathrm{msec}$ is achieved when the downstream pressure is allowed to rise rapidly from $2.4 \times 10^{-7}$ Torr to $1 \times 10^{-4}$ Torr.

FE Vacuum: Because the FEs are directly coupled to the storage-ring vacuum system, the upstream $\mathrm{FE}$ vacuum requirements equal those of the storage ring, i.e., a pressure of 1 nTorr or less with beam on. Achieving UHV conditions requires that hydrocarbons and contaminants be kept to a minimum. Information on UHV pressure is monitored through the use of nude UHV ion gauges, and higher pressure information is obtained through the use of convectron gauges. Mass spectra are also monitored at the downstream end of the FEs, where a residual gas analyzer (RGA) is installed.
The vacuum valve positions are monitored by the EPS, which also monitors the vacuum levels upstream and downstream of each valve. The valves are commanded to close upon detection of a vacuum leak but only if they are protected from direct photon radiation by a photon shutter or an EPS-commanded stored-beam abort. Vacuum breach tests performed on FEs (discussed in Chapter 4, section 4.2) have shown that the FE components perform to specifications.

FE Cooling System: Each FE includes two fixed masks and two photon shutters. These masks and shutters are capable of handling large thermal loads. These components are water cooled. The storage ring consists of two different closed-loop water systems. One is used for components made of aluminum, and the other is for all copper-based components. The water is deionized with a minimum resistivity of 3 megohms. The inlet pressure of the water system is maintained at about $150 \mathrm{psi}$, while the return is kept at 40 psi. All FE components use deionized water.

In the case of ID FEs, the masks and shutters are made of Glidcop and the water channels are fitted with copper mesh for efficient heat transfer. Because of the mesh in these channels, the pressure drop across each of the components is significant and is measured across each unit. The flow rate is measured for all components.

The pressure drop across a component is measured by using a transmitter. The transmitter is located outside the storage ring to minimize the damage due to high radiation levels. The signals from the transmitter are 4-20 mA current loops. These signals are fed to interface controllers, which are devices that read the current signal and display the flow in inches of water and the pressure in pounds per square inch. These controllers have alarm set 
points and have level outputs, as well as RS485 communication capability.

\subsubsection{Equipment Protection System}

\section{Introduction}

The APS has overcome a number of design challenges in engineering the $\mathrm{FE}$ and beamline components so that they will not be damaged by the thermal loads produced by high powerdensity $\mathrm{x}$-rays. Another major goal is to ensure that the storage-ring vacuum is not compromised under any vacuum-failure scenario in the FE or beamline.

The FE Equipment Protection System (FE-EPS) monitors and controls devices located in the beamline FEs. Actions taken depend largely on the severity of the fault, ranging from merely setting an alarm, to closing shutters and valves, to inhibiting stored beam. One of the major considerations driving system design was to limit beam aborts and thus contribute to higher operating efficiency of the facility.

Fail-safe principles are incorporated into the design, and the system will lapse into a predetermined safe condition (deenergized to trip) following a failure, including loss of power, air-pressure drop, drop in water flow, shorted outputs, and open circuits.

\section{System Overview}

Programmable Logic Controllers (PLCs) are used to handle all system monitoring, control, troubleshooting, and reporting functions.
PLCs allow for the design of a very advanced interlock and control system that can handle a large number of distributed $\mathrm{I} / \mathrm{O}$ points. Each FE is provided with an autonomous EPS that monitors the following parameters: cooling water flow and differential pressure, temperature, vacuum, pneumatic pressure, photon and safety shutter positions, positions of vacuum valves, and status of the systems to which the FE-EPS interfaces. The main FE elements monitored and controlled by the FE-EPS are shown schematically in Fig. 2.5.

\section{The Programmable Logic Controllers}

A state-of-the-art platform technology AllenBradley processor PLC-5/30 is used. The PLC performs a complete run-time checksum on the control algorithm and sets a fault bit if an invalid checksum is detected. Should this be the case, the system responds by closing FE critical devices and aborting the particle beam.

\section{Interfaces}

In order to isolate different power systems, all interfaces between the FE-EPS and other systems and subsystems are implemented through relay contacts. These interfaces are listed in Table 2.1.

\section{Analog Signals}

Analog signals are not wired directly to PLC input modules. Instead, microprocessor-based process controllers are used. The controllers accept 4-20 mA current-loop signals from the differential pressure transducers used to 


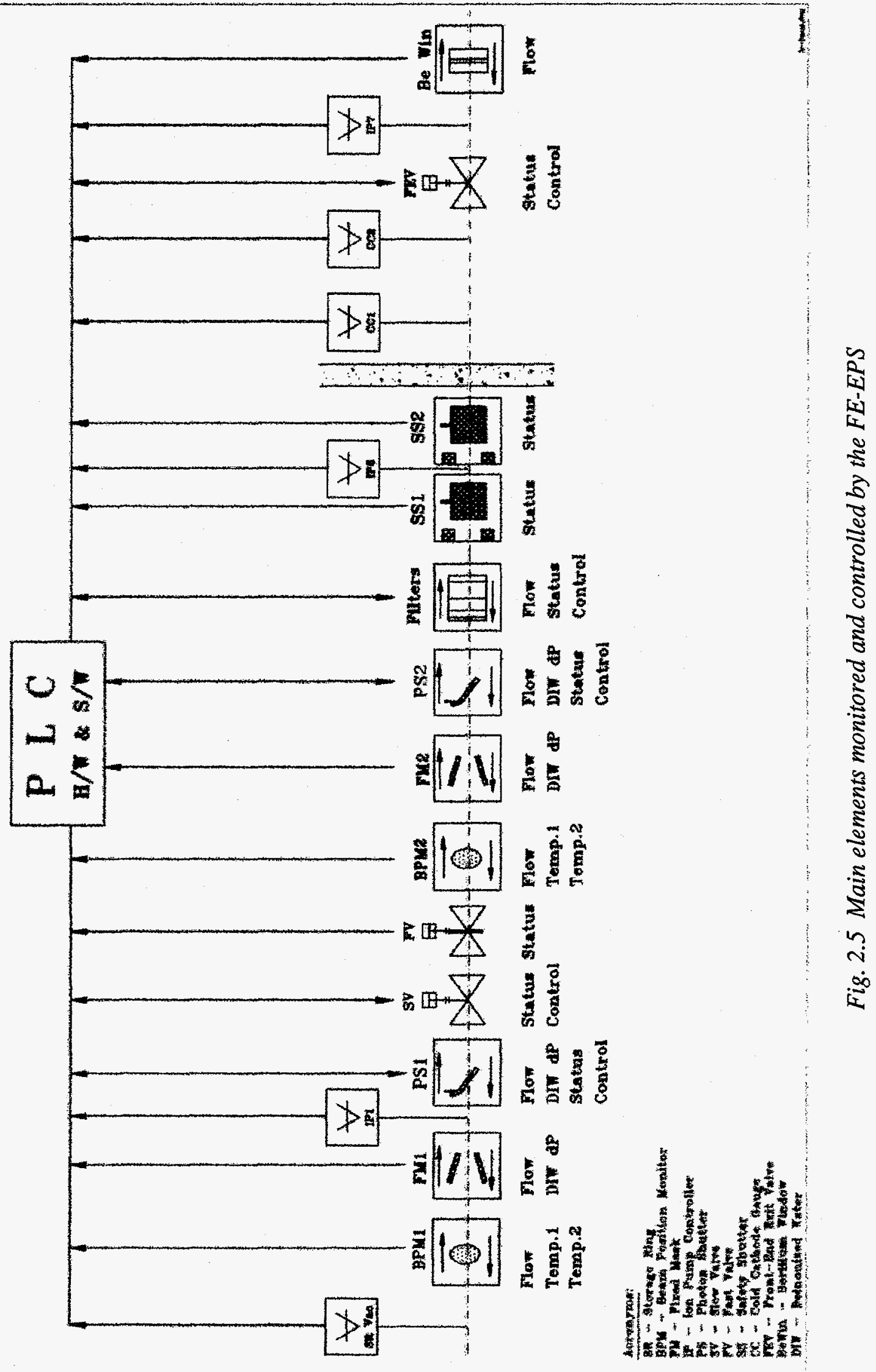


Table 2.1 EPS Interfaces and Functions

\begin{tabular}{|l|l|}
\hline \multicolumn{1}{|c|}{ System } & \multicolumn{1}{c|}{ Signal Functions } \\
\hline Personnel Safety & $\begin{array}{l}\text { Monitor shutter-open positions } \\
\text { Control of photon shutters }\end{array}$ \\
\hline Insertion Device & $\begin{array}{l}\text { Monitor gap for "max open" status } \\
\text { Control emergency gap open }\end{array}$ \\
\hline Storage Ring Personnel Safety & $\begin{array}{l}\text { Monitor shutter-closed positions } \\
\text { Monitor global online/offline }\end{array}$ \\
\hline Vacuum System & $\begin{array}{l}\text { Monitor SV, FV, CC1, CC2 status } \\
\text { Control SV and FEV } \\
\text { Permit vacuum controller operation in local mode }\end{array}$ \\
\hline Beamline Interlocks & $\begin{array}{l}\text { Monitor interlock summation signal and request to close FEV } \\
\text { Send FE interlock status, shutter and FEV status }\end{array}$ \\
\hline Storage Ring Machine Protection & Control permission to run storage ring RF \\
\hline
\end{tabular}

measure deionized water parameters and $\mathrm{mV}$-level signals from the thermocouples. Use of the process controllers allows us to change setpoints without altering PLC code and to locally monitor input values from the controller front panel. Each system variable requires its own controller. Controllers are equipped with an RS485 interface, which is used for data collection.

\section{Machine Protection System Interface}

One of the challenges faced in the design was the method of protecting the fast vacuum valve from synchrotron radiation when the valve is triggered to close. The valve closure time is less than $7 \mathrm{msec}$. The PLC alone is not fast enough. The solution is an electronic circuit that interfaces to the vacuum-valve controller, PLC, and storage-ring machine protection system and is capable of aborting storage ring beam in $\sim 2 \mathrm{msec}$ by interrupting a $1 \mathrm{MHz}$ unipolar pulse train.

\section{Vacuum System Controller Interface}

A vacuum system controller is capable of monitoring cold cathode gauges (CC) and controlling UHV valves. It can successfully operate as a stand-alone system in a laboratory setting. However, for this application, its capabilities are too limited. By integrating the controller with the PLC, higher system flexibility was achieved, making the controller more intelligent and reducing cost.

\section{Reporting}

The status information of the FE-EPS is being incorporated into the APS control system, which is based on EPICS (Experimental Physics and Industrial Control System). This will allow monitoring of the interlock system from the control room and various other locations via operator interface (OPI) consoles. The OPI screens can be called up from authorized X terminals or UNIX workstations. 
Reporting is through a one-way communication protocol, from PLC to APS control system, which allows for displaying EPS status without concern about corrupting the PLC input data table. Graphical displays will include an overall view of the ring and FEs, as well as zoom-in screens for each interlock system. Graphical displays of trending and tabular data of actual values of the transducers (flow, temperature, etc.) are also being planned and developed.

\section{Summary}

Currently 36 FE-EPSs are instrumented and operational, and additional systems are being brought on line on a regular basis. The FEEPS on the BM beamline in Sector 1 has been in operation for ten months. This period has provided valuable operating experience. For example, a number of time delays were finetuned, and a $5 \mathrm{sec}$ timer associated with the water interlocks and beam abort was programmed in. Prototypes of the systems have been carefully tested and debugged offline. Trips observed were all for "legitimate" reasons; most were caused by decreases in the cooling water flow rate to values below the predetermined range. The systems responded the way they should, and there appear to have been no unexplained trips.

\subsubsection{The APS Personnel Safety System}

\section{Introduction}

The APS is designed to operate a maximum of 70 experimental beamlines concurrently. Each beamline includes several shielded experiment stations. Personnel access into these stations is controlled during beamline operation via the APS/XFD PSS. The PSS is an engineered safety system that interlocks personnel access to these stations with $\mathrm{x}$-ray beam-off conditions via beam shutter operation and, if required, storage-ring operation.

The PSS for each beamline is interfaced directly with the Accelerator Systems Access Control and Interlock System (ACIS) to allow the disabling of storage-ring operation. Each beamline has a dedicated PSS that is isolated from all other beamlines to prevent a fault in one beamline from affecting the PSS of other beamlines.

Although there are a variety of beamline designs that reflect the types of experiments being done at the APS, basic PSS configuration and control functions remain the same. If required, specialized user control panels are incorporated into the standard library of PSS hardware.

The PSS is designed to comply with accelerator safety standards in DOE orders and other relevant good practices for accelerator facilities. Among the requirements derived from the above criteria, to which the PSS conforms, some of the more important items are as follows:

- The system is designed to be fail-safe, so that common failure modes leave the PSS in a safe, beam-off state.

- The designs incorporate redundant protection, ensuring that no single component or subsystem failure leaves the PSS in an unsafe condition. 
- Provisions for testing are included, so the proper component and system function may be verified.

- Access and egress controls are incorporated so that personnel are not exposed to $\mathrm{x}$-ray radiation. These include emergency shut-off devices, status signs, search and secure procedures, and emergency exit mechanisms.

- A strict configuration control system protects documentation, circuits and software against unauthorized and inadvertent modification. Critical devices are clearly labeled to note that tampering is strictly forbidden.

\section{PSS Description}

The PSS provides system-wide (redundant) access control by employing two independent levels of interlock protection, referred to as chain A and chain B. Reduction of common cause failures is achieved by the use of these redundant chains and diversity in the implementation and functionality of the two chains. The system is tested every six months to ensure proper operation.

The PSS protective logic function is implemented in the form of PLCs. A summary of the PSS functionality is illustrated in Fig. 2.6. Using different types of PLCs for each independent chain provides hardware diversity in the PSS. Diversity of PSS software is achieved by using a different language for each chain. Complexity in APS beamline operating modes is translated to the PSS logic. Thus, PLCs were chosen as the

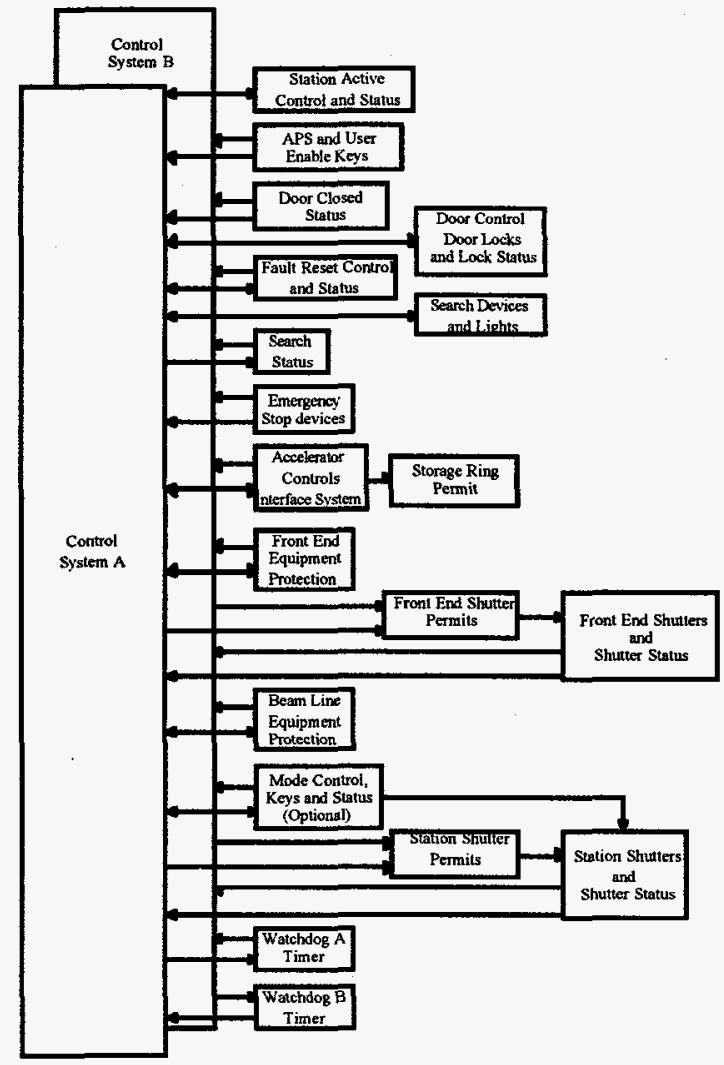

Fig. 2.6 Summary of PSS functionality

PSS hardware platform because they are ideally suited to provide complex protective logic.

The PSS incorporates the following equipment in addition to the PLC hardware:

\section{FE PSS hardware}

- Interfaces to the ACIS (for storagering shutdown), FE-EPS, beamline EPS, and EPICS (for monitoring and status)

- Interfaces to critical devices that shut down or mitigate the radiation hazards 
- Uninterruptible power supply to protect against short-term AC power loss

- Equipment racks, conduit, cable trays and cables

\section{Beamline PSS hardware}

- User panels and status displays

- Door position sensors (mechanical and magnetic)

- Door locking mechanism

- Search-and-secure boxes

- Emergency beam stop

- Visible and audible warning indicators

- Interfaces to critical devices that shut down or mitigate the radiation hazards

- Equipment racks, conduit, cable trays and cables

The PSS also provides for external watchdog timer signals between the two PSS chains. If this "heartbeat" signal skips a beat, the PSS will cause a stored-beam abort.

Clearly, the user interface with the PSS involves only beamline hardware. Beamline PSS equipment (to which the user needs access) falls into two categories: user control panels and station hardware. PSS panels, such as door access controls, shutter controls, and mode controls, are in equipment cabinets located on the outside of the experiment stations. These PSS cabinets are locked to prevent uncontrolled access to wiring, but the fronts are open to allow operation of the control panels. These cabinets also contain PLC I/O hardware for each PSS chain. The PSS beamline control panels provide the following user functions:

- a logical visual indication of the beamline status

- beamline shutter control

- the means to switch shutter control among different stations on a beamline

- the means to change PSS beamline operating modes

- the means to reset PSS faults

- the mechanisms to administratively take stations off-line and bring them back on-line.

The most used of the PSS control chassis is the station control panel, a typical layout of which is shown in Fig. 2.7. This panel is used to open and close safety shutters and provide visible feedback regarding the status of the accelerator, other experiment stations, and the shutters.

The status display consists of a chain of LED indicators laid out in a logical fashion consistent with the beamline layout. Lamps are green if the related devices or systems permit the beam to be propagated down the beamline and are red if configured to stop the beam. 


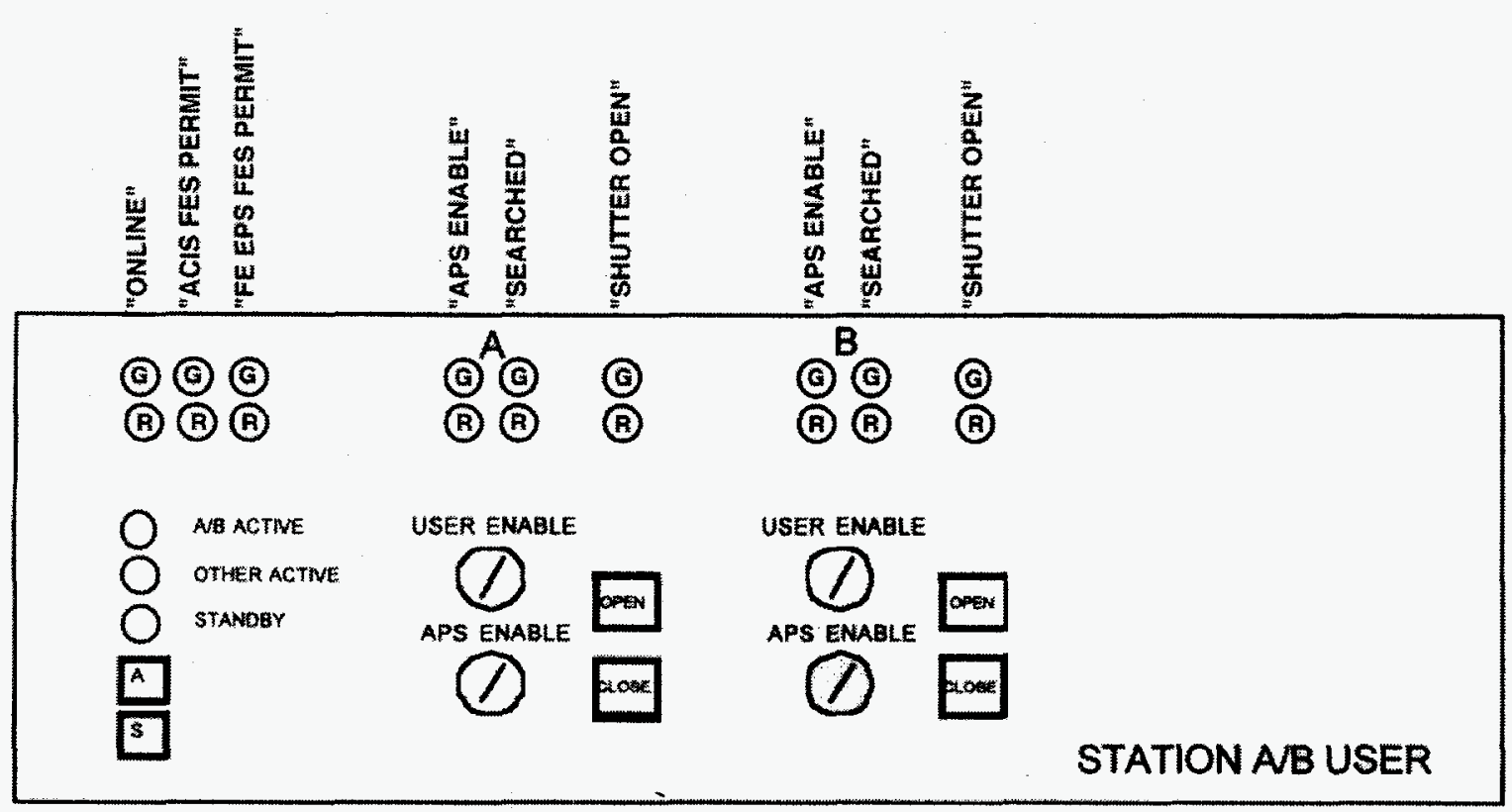

Fig. 2.7 Typical layout of a station control panel

The PSS station hardware provides for emergency beam shutoff and emergency egress. A station search and secure must be completed before beam is transported into a station. During this procedure, the user is forced to depress PSS search buttons in a predetermined sequence and close the station doors within a given time period in order for the PSS to consider the station as secure for beam. Visual and audible indicators are given during the search process. The locations and numbers of search buttons, emergency beam stop buttons, and door open/close buttons are determined by internal XFD safety reviews. At the completion of a successful search, the safety shutters are activated if the other interlock conditions are satisfied. Typical PSS protective logic conditions that need to be completed before opening the safety shutters are shown below:

- Access doors closed

- Station search and secure completed
- Emergency interlocks complete

- Beamline mode selected

- Permits from EPS complete

- APS and user enable keys in a permissive state

- APS storage-ring shutter permit complete

\subsubsection{Controls}

At the APS, all controls are standardized with EPICS. This system consists of equipment interfaced to VME-based hardware. The VME crate, normally called the IOC (input output controller), talks to the computers via the ethernet. With EPICS, access to the controls is available from any computer located on the 
same network. This scheme has a great degree of flexibility.

Because of the flexibility provided by EPICS, all data are available at any time to anyone with access to the computer network. There is a separate subnet with restricted access for all the control systems. To provide an added safeguard against unwarranted access to the IOCs, they are all located on this subnet. With the location of an EPICS gateway, data from the control system are provided to other subnets.

In a typical FE, EPICS can currently only read and is not allowed to control. All control actions for the FE have to be performed at locations on top of the storage ring. All vacuum pump and gauge controllers are interfaced with EPICS. The interface enables the ion pump current and the vacuum to be read continuously. All water flow and pressure systems are also interfaced to EPICS via the RS485 interface available in the interface controllers. All data from EPICS are constantly logged to provide for later analysis of specific trends. The constant monitoring of data provides advance warning so that preemptive action can be taken to avoid failures.

The XBPM raw voltage signals for the current amplifier are interfaced to the control system via an RS485 interface. The normalization of the raw signals is performed in the IOC. All signals, both raw and normalized, are available via EPICS.

The ID control is also implemented with EPICS. The ID motor controllers are commanded by EPICS, and the encoders are used to read the precise position of the device.
Using the EPICS gateway, added security is provided for ID control to the specific users of a particular beamline.

The PSS and FE-EPS operator/user interface (OUI) is provided for APS facility use. The remote OUI for PSS and FE-EPS has the capability to interface with EPICS. User screens have been developed that graphically represent the PSS status, and the remote OUI does not control any PSS functionality.

\subsection{Operations Performance and Reliability}

\subsubsection{Installation Status}

The APS storage-ring design incorporates a magnetic lattice with 35 5-meter-long straight sections available for installation of IDs. The design also incorporates the necessary beam ports for extracting radiation from 35 of the 40 BMs. With each sector containing an ID beamline and a BM beamline, the APS can accommodate a total of 35 sectors. The funding for the APS Project included the funds to construct 20 sectors worth of FEs and IDs available for user research and an additional sector for particle beam diagnostics studies by the APS facility. Installation of IDs and FEs in these sectors is continuing and will be completed by July 1997. A summary of the installation status is shown in Table 2.2. The installation activities are well ahead of the user beamline construction and have not impeded the user beamline commissioning schedule. The remaining 14 IDs and 28 FEs will be built and installed as future funding becomes available. 
Table 2.2 Installation and Commissioning Status Summary for User IDs and FEs as of March 1997

\begin{tabular}{|l|c|c|c|c|c|}
\hline & $\begin{array}{c}\text { Design } \\
\text { maximum }\end{array}$ & Planned & Installed & Commissioned & Comments \\
\hline IDs & 34 & 20 & 17 & 16 & includes 1 wiggler and 1 EMW \\
\hline ID vacuum chambers & 34 & 20 & 20 & 20 & \\
\hline ID front ends & 34 & 20 & 17 & 16 & \\
\hline BM front ends & 34 & 20 & 19 & 18 & \\
\hline
\end{tabular}

User beamline installation continues at a rapid pace. Although the installation schedule is primarily governed by user funding availability, APS personnel are responsible for managing the installation contracts of the experiment stations and beamline utilities. As of March 1997, 39 experiment stations have been completed on 22 beamlines. Of these 22 beamlines, all have had x-rays delivered to at least the FOE. The dates for the start of commissioning, for each of these beamlines, are shown in Table 2.3. Another 36 stations are nearing completion, and another 23 are at some stage of construction. The current beamline status is shown in Fig. 2.8. By the end of calendar year 1997, all 98 currently planned stations will be completed and all 40 beamlines are expected to be either under commissioning or in operation. Some of the beamlines will not be fully implemented with their full capacity of experiment stations, and as user funding becomes available, additional stations will continue to be constructed on the existing beamlines.

The PSS (as described in section 2.2.5) is the responsibility of XFD personnel. This includes the installation, initial system validation of proper operation, and subsequent revalidations each time an additional station is

\section{Table 2.3 Dates of First Commissioning of APS Beamlines}

\begin{tabular}{|l|l|}
\hline Beamline & Date of First Beam \\
\hline 1-BM & $3 / 26 / 95$ \\
\hline 1-ID & $8 / 9 / 95$ \\
\hline 2-BM & $6 / 24 / 96$ \\
\hline 2-ID & $3 / 26 / 96$ \\
\hline 3-ID & $1 / 24 / 96$ \\
\hline 5-BM & $3 / 27 / 96$ \\
\hline 5-ID & $5 / 22 / 96$ \\
\hline 7-ID & $8 / 16 / 96$ \\
\hline 8-ID & $8 / 17 / 96$ \\
\hline 10-ID & $8 / 8 / 96$ \\
\hline 11-ID & $1 / 14 / 97$ \\
\hline 12-BM & $3 / 26 / 96$ \\
\hline 12-ID & $5 / 20 / 96$ \\
\hline 13-BM & $9 / 17 / 96$ \\
\hline 13-ID & $9 / 27 / 96$ \\
\hline 17-BM & $10 / 14 / 96$ \\
\hline 17-ID & $7 / 5 / 96$ \\
\hline 19-BM & $6 / 25 / 96$ \\
\hline 19-ID & $3 / 26 / 96$ \\
\hline 20-ID & $12 / 18 / 96$ \\
\hline 33-ID & $7 / 3 / 96$ \\
\hline 35-ID & $3 / 6 / 97$ \\
\hline & \\
\hline
\end{tabular}


APS - CAT BEAMLINE STATUS - MARCH 1997

VACUUM

FRONT-

CHAMBER

END

EXPERIMENT

STATIONS

ØX Lengt DW-Periog BM ID

\begin{tabular}{|c|c|}
\hline SECTOR & CAT \\
\hline 1 & SRI \\
\hline 2 & SRI \\
\hline 3 & SRI \\
\hline
\end{tabular}

$8 \mathrm{~mm} \times 5 \mathrm{~m} \quad \mathrm{U}-33 \mathrm{~mm}$

BM

ID

\begin{tabular}{|c|c|}
\hline 4 & \\
\hline 5 & DND \\
\hline 6 & MU \\
\hline
\end{tabular}

$8 \mathrm{~mm} \times 5 \mathrm{~m} U-33 \mathrm{~mm}$

$8 \mathrm{~mm} \times 5 \mathrm{~m} \quad \mathrm{U}-27 \mathrm{~mm}$

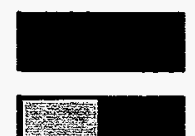

\begin{tabular}{|l|l|l|l|l|l|l|l|l|l|l|l|l|}
\hline$A$ & $B$ & $C$ & $D$ & $E$ & $B$ & $C$ & $D$ & $E$ \\
\hline
\end{tabular}
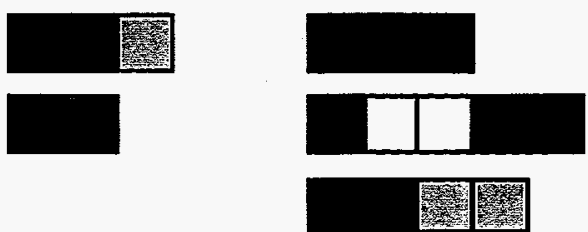

\begin{tabular}{|l|l|}
\hline $\mathbf{7}$ & MHATT \\
\hline $\mathbf{8}$ & IMM \\
\hline
\end{tabular}

$8 \mathrm{~mm} \times 5 \mathrm{~m} U-33 \mathrm{~mm}$

$8 \mathrm{~mm} \times 5 \mathrm{~m}$
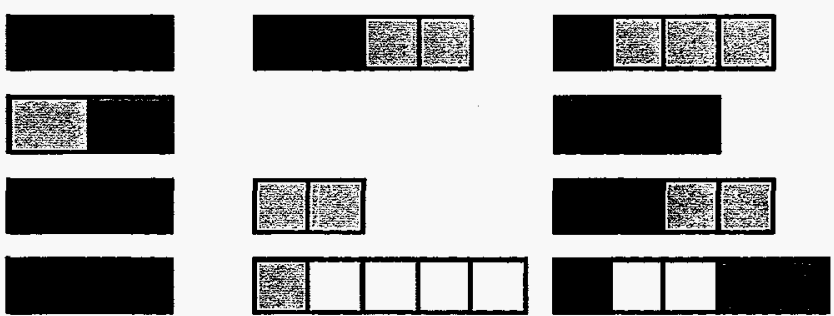

\begin{tabular}{|l|l|}
\hline $\mathbf{9}$ & CMC \\
\hline
\end{tabular}

$8 \mathrm{~mm} \times 5 \mathrm{~m}$

$\mathrm{U}-33 \mathrm{~mm}$

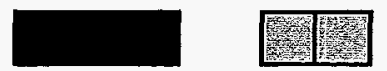

$8 \mathrm{~mm} \times 5 \mathrm{~m} \quad \mathrm{U}-33 \mathrm{~mm}$

$8 \mathrm{~mm} \times 5 \mathrm{~m} \quad \mathrm{U}-33 \mathrm{~mm}$
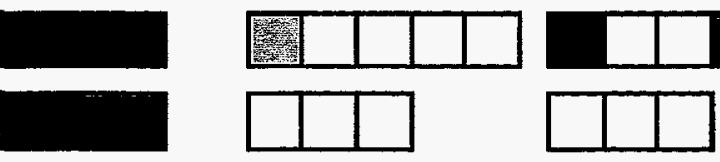

\begin{tabular}{|l|l|}
\hline 10 & MR \\
\hline
\end{tabular}

$8 \mathrm{~mm} \times 5 \mathrm{~m} \quad \mathrm{U}-33 \mathrm{~mm}$
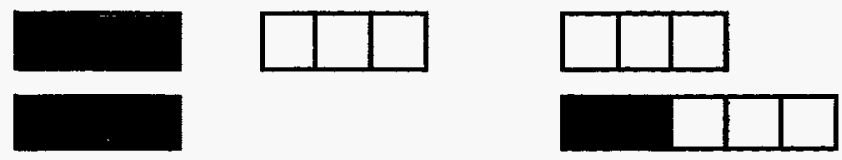

\begin{tabular}{|l|l|}
\hline 11 & BESSRG \\
\hline
\end{tabular}

SPECIAL

EMW

\begin{tabular}{|c|c|}
\hline 12 & BESSRC \\
\hline
\end{tabular}

$8 \mathrm{~mm} \times 5 \mathrm{~m} \quad \mathrm{U}-33 \mathrm{~mm}$

\begin{tabular}{|l|l|}
\hline 13 & GEOCARS \\
\hline
\end{tabular}

$8 \mathrm{~mm} \times 5 \mathrm{~m} \quad \mathrm{U}-33 \mathrm{~mm}$
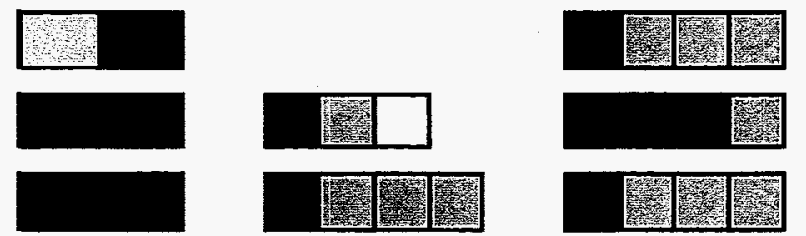

\begin{tabular}{|c|c|}
\hline 14 & BIOCARS \\
\hline 15 & CHEMCA \\
\hline
\end{tabular}

$12 \mathrm{~mm} \times 2.5 \mathrm{~m} W-85 \mathrm{~mm}$
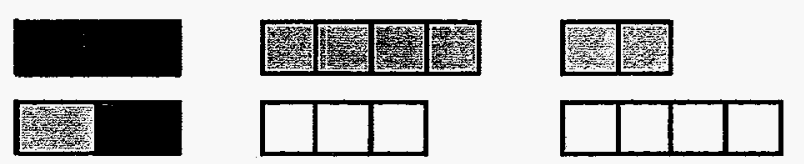

\begin{tabular}{|c|c|}
\hline 16 & \\
\hline 17 & IMCA \\
\hline 18 & BIO \\
\hline 19 & SBC \\
\hline 20 & PNC \\
\hline 33 & UNI-1 \\
\hline 34 & UNI-2 \\
\hline
\end{tabular}
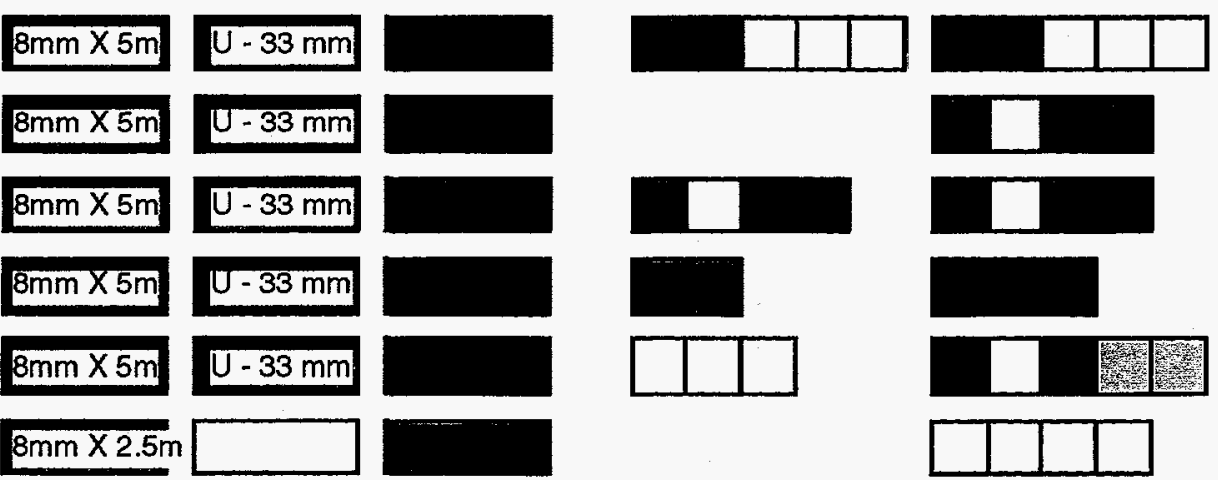

Commissioned

Being

Commissioned Commissioning

Being Installed

Fig. 2.8 APS CAT beamline status 
added to the beamline, each time the control software is modified, or at six-month intervals as required by DOE Order 5480.25 . The number of user stations that have been instrumented with the PSS is shown in Fig. 2.9. The figure also shows the number of revalidations performed and summarizes the planned future activities.

\subsubsection{Operations Experience}

The storage-ring commissioning was begun by ASD on the morning of February 20 , 1995 , at 5:53 a.m., when a $7 \mathrm{GeV}$ particle beam from the synchrotron was first injected into the APS storage ring. A period of installation and storage-ring commissioning followed. The commissioning consisted of checking out the operation of various systems, adjusting the injected beam orbit, and conducting beam-loss studies. The commissioning activities were limited to night and early morning hours to avoid interfering with construction activities. During this early period of commissioning, adequate radio frequency (rf) voltage was unavailable for storing the beam at $7 \mathrm{GeV}$. Therefore, the initial stored beam attempts were conducted at an energy of $4.5 \mathrm{GeV}$. On March 25 at 1:55 a.m., the first stored beam of electrons with a measurable lifetime inside the storage ring was achieved.

At 7:13 a.m. on Sunday, March 26, 1995, the first $x$-rays from a $B M$ source were delivered down the Sector 1 BM beamline (1-BM) and into station 1-BM-A (FOE). This major milestone took place not only during the centennial of Roentgen's discovery of X-rays but also within a day of the 150th anniversary of his birth. During the subsequent operating periods, the methodology for measurement of the station shielding integrity was developed. The process is described in more detail later. (See "Shielding Validation of the Experiment Stations" in section 2.4.5.)

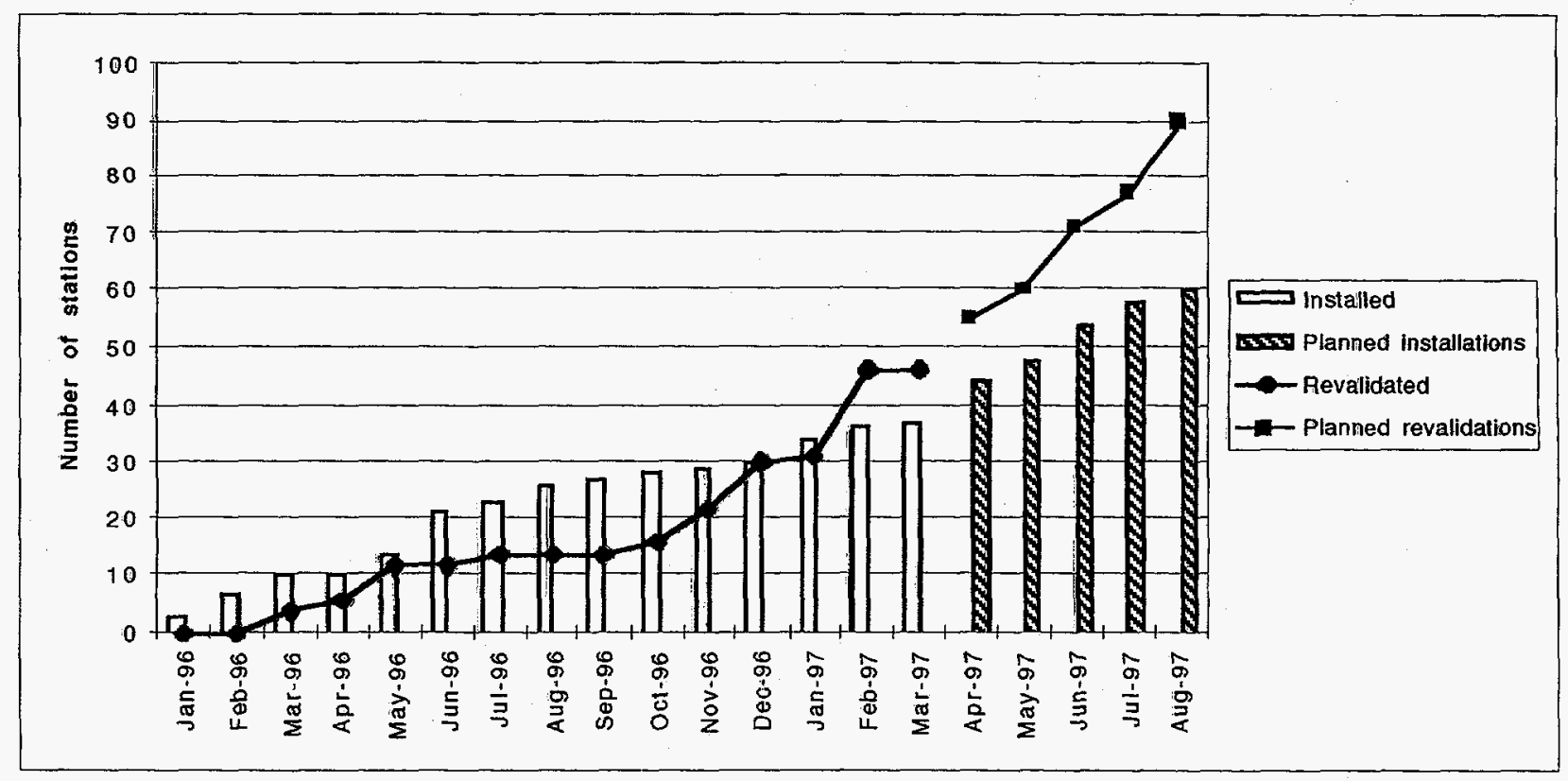

Fig. 2.9 Number of PSS installations and revalidations (completed and planned) 
During July and early August of 1995, the first ID vacuum chamber and an undulator $A$ were installed in Sector 1 of the APS storage ring. The vacuum chamber was an initialphase chamber with a vertical aperture of $12 \mathrm{~mm}$ and a minimum possible undulator gap of $14.5 \mathrm{~mm}$. The continued installation of the rf system had also provided adequate voltage for storing the beam at the full energy of $7 \mathrm{GeV}$. The successful operation of the storage ring in this configuration delivered the first undulator beam to the experiment hall floor of the APS. On August 9, 1995, the photon shutters in the first ID beamline FE were opened, and the first undulator beam was delivered to the FOE on the experiment hall floor. X-rays from undulator $A$ were observed on a fluorescent screen.

After measurement of the shielding integrity of the station, the first experiments looked at the effect of the undulator on a stored $1 \mathrm{~mA}$ particle beam. Measurements of the closedorbit distortion showed that the performance of the undulator exceeded specifications. The results were gratifying because they showed that the undulator fabrication and magnetic tuning had fully met the magnetic field tolerances.
Using electrons, $100 \mathrm{~mA}$ of current was stored for the first time on January 26, 1996. On July 31, 1996, $100 \mathrm{~mA}$ of current was stored using positrons.

Since that time, a total of 22 beamlines have had the first x-rays delivered to at least the FOE. All beamline work is still at an early stage, consisting primarily of commissioning beamline components, such as monochromators, mirrors, detectors, etc. A few of the stations have conducted scientific research to demonstrate the potential of the APS.

The operating schedule has been optimized to provide adequate and effective time for continuing installation activities, as well as providing time for accelerator studies and user beamline commissioning activities. The storage ring has operated at a $50 \%$ duty cycle, with a nominal 3-week-on and 3-week-off cycle. Approximately $30 \%$ of the operating time is provided for accelerator studies, with the remainder used for beamline commissioning. Detailed recordkeeping of operating data was started in June 1996. Table 2.4 provides information about the user operating periods (runs) since that time.

Table 2.4 User run statistics

\begin{tabular}{|l|l|c|c|c|c|c|c|}
\hline Run designation & \multicolumn{1}{|c|}{$96-4$} & \multicolumn{1}{|c|}{$96-5$} & $96-6$ & $96-7$ & $97-1$ & $97-2$ & \\
\hline $\begin{array}{l}\text { Run start date/ } \\
\text { end date }\end{array}$ & $\begin{array}{l}6 / 22 / 96- \\
7 / 7 / 96\end{array}$ & $\begin{array}{l}8 / 5 / 96- \\
8 / 19 / 96\end{array}$ & $\begin{array}{l}9 / 17 / 96- \\
10 / 20 / 96\end{array}$ & $\begin{array}{l}12 / 10 / 96- \\
12 / 23 / 96\end{array}$ & $\begin{array}{l}1 / 7 / 97- \\
1 / 25 / 97\end{array}$ & $\begin{array}{l}2 / 25 / 97- \\
3 / 9 / 97\end{array}$ & \\
\hline $\begin{array}{l}\text { Number of } \\
\text { scheduled hours }\end{array}$ & 300.0 & 324.0 & 596.0 & 268.0 & 376.0 & 296.0 & $\Sigma=2160.0$ \\
\hline $\begin{array}{l}\text { Number of } \\
\text { available hours }\end{array}$ & 239.8 & 201.9 & 456.0 & 183.0 & 264.8 & 206.0 & $\Sigma=1551.5$ \\
\hline Availability & $79.9 \%$ & $62.3 \%$ & $76.5 \%$ & $68.3 \%$ & $70.4 \%$ & $69.6 \%$ & $\overline{\mathrm{x}}=71.8 \%$ \\
\hline
\end{tabular}


The normal operating mode for user operation is to start with the storage ring filled to $100 \mathrm{~mA}$. Under the present storage-ring operating conditions, the average lifetime at this current is $15-20$ hours. The stored beam is dumped when the stored current reaches $50 \mathrm{~mA}$. Storage-ring availability has not yet reached its goal of $>90 \%$, with storage-ring faults (primarily from the rf system) often causing beam dumps prior to reaching the $50 \mathrm{~mA}$ level. This is reflected in the data shown in Fig. 2.10, which shows the average number of beam fills per day. The distribution of fill duration for each of the runs is shown in Fig. 2.11. Reliability issues relative to XFD components are discussed in the next section.

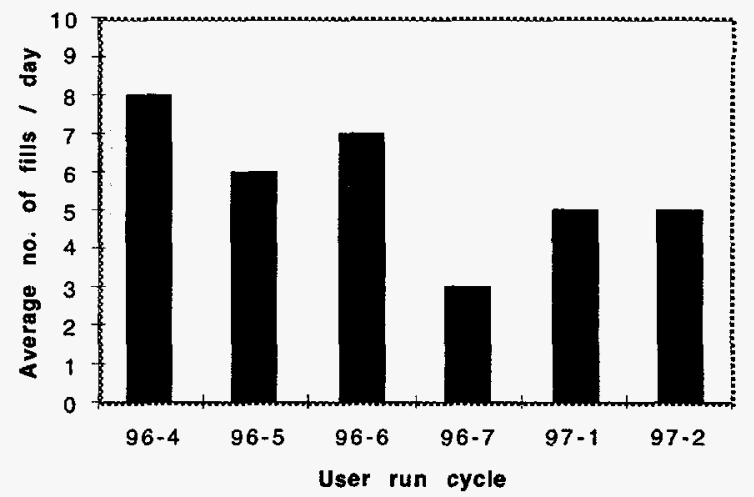

Fig. 2.10 Average number of storage-ring beam fills per day

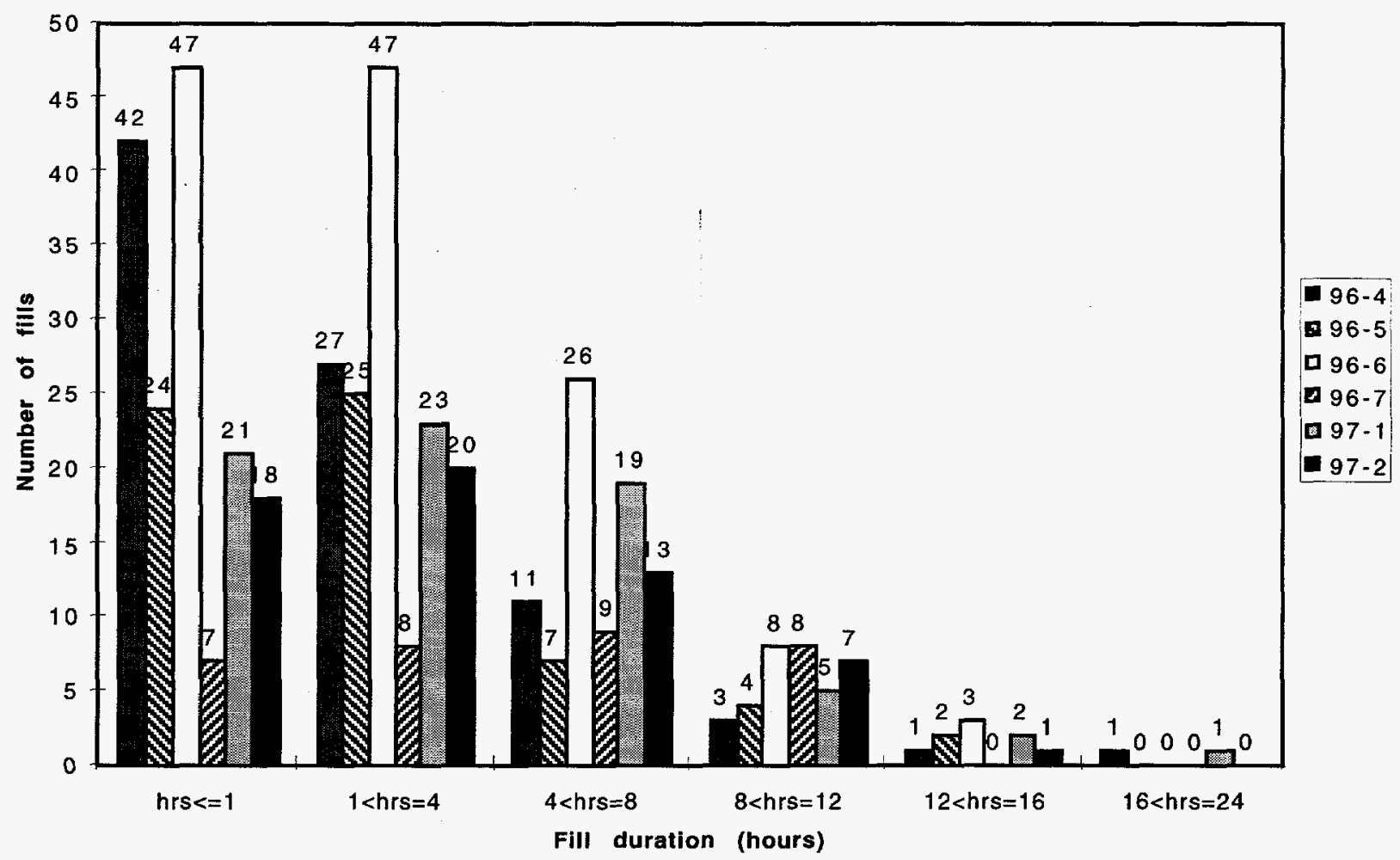

Fig. 2.11 Number of fills with indicated fill durations for the runs shown in Table 2.4 


\subsubsection{Reliability Studies and Analysis}

Statistical data gathering for the APS beamlines began during the third quarter of CY 1996, when an effort was initiated to monitor the reliability of the operational systems down to the component level. This effort is targeted at achieving the following goals:

- Increase availability of beam time to the user community

- Minimize failures and prevent their recurrence

- Predict failures before occurrence

Failures or malfunctions of any equipment required to directly support or operate the beamlines, or which is related to personnel or equipment safety, must be reported and tracked to resolution. Failures are diagnosed and followed up by the cognizant individuals and tracked/analyzed by the quality assurance reliability engineers.

The data required to achieve the abovementioned goals are gathered by two methods:

1. The controls for monitoring the system performance of the FEs and IDs are located on top of the storage ring with an EPICS interface. All vacuum pump and gauge controllers are interfaced with EPICS. The interface enables the ion pump current and the vacuum to be read continuously. Water flow and pressure systems are also interfaced to EPICS.
All system/component performance data from EPICS are constantly logged and monitored. This facilitates data gathering and analysis of specific trends and provides the flexibility for advance warning on failures. Problems can then be dealt with in a proactive manner.

2. The life history of each critical component is maintained in the equipment tracking system (ETS), a database system designed to archive key information on beamline critical components. Component data can be entered either electronically with a scanner or manually with a computer and keyboard. In the case of a scanner, unique equipment numbers are generated using ETS and printed onto tags in the form of barcodes. The barcodes are then placed on the component and scanned into the computer. If tag mutilation is possible (due to component bakeout, etc.), the barcode number is engraved on the part directly, and the information is entered manually.

Types of stored information for the components include manufacturer name, component location, drawing number, description, serial number, purchase order number, delivery date, warranty, calibration $\log$, repair $\log$, nonconformance history, and maintenance $\log$. With this information, the ETS can keep a complete history of each individual component from incoming inspection to failure and/or removal from service. In addition, users can be notified of maintenance and calibration requirements of components when applicable. Hard-copy reports of all data are also available to make analysis much easier and more useful. 
This database is currently being adapted for use on the APS facility Web pages. The main benefit of this exercise is to make the data more accessible for facility personnel. On the Web, a complete FE or ID component list will be available on-screen simply by clicking on the appropriate sector prompt. A click on a specific component in the list will cause a pop-up window to appear with more detailed information on the component.

Another benefit of adapting ETS to the Web will be the ability to connect the ETS database fields with current XFD Operations Organization Web sites. The XFD Operations Organization is currently employing a failure reporting system called "trouble reports" on the Web. Trouble reports are generated when any problem arises during normal operations. Eventually, the information logged in the trouble reports will be automatically downloaded into the repair $\log$ for the specific components in the ETS. Analysis of this repair data will then be possible and could lead to failure correlations.

In the future, it will also be possible to have the ETS determine maintenance schedules for beam shutdown periods. This could be performed by tying in the ETS maintenance information to a scheduling program.

To date, 32 FEs and 17 IDs are in the operational stage. Failure or malfunction data have been collected on these systems by XFD personnel throughout the construction, installation, and operation phases. The data have been organized into two main groups: (1) critical component rejections during incoming inspection, and (2) failures after component installation. Rejections during incoming inspection are documented via ANL Nonconformance reports and are also entered into the XFD ETS database. Component anomalies after installation are logged in the Web-based XFD Operations trouble reporting system.

Although the operational phase of the APS beamlines is still at an early stage and the numbers of failures documented thus far show no signs of any trends, the failures detected to date are nonetheless being analyzed. For the operational analysis, only components that failed and were either removed from service or repaired and reinstalled are included.

The graph in Fig. 2.12 shows both the number of rejections at incoming inspection and the failures during operations for each quarter starting with the second quarter of 1996. Rejections at incoming inspection do not appear after quarter II, 1996, because the majority of critical components for the FEs and IDs were already inspected at that time. A manufacturing problem with the front-end pressure transmitters led to rejection of 380 units accounting for $41 \%$ of the total rejections.

Failures after installation fall into three main categories: mechanical, electrical, and vacuum. Electrical and mechanical failures are the most prevalent, occurring in devices such as PSS/EPS modules, encoders, controllers, and pressure transmitters. To a lesser extent, vacuum leaks have been recognized on $\mathrm{FE}$ equipment as well.

Analysis of the statistical data gathered from five beam running cycles indicates the following:

1. With a total of 1860 hours of running time, the XFD Operations systems 


\section{Statistical Data on Beamline Component Performance}

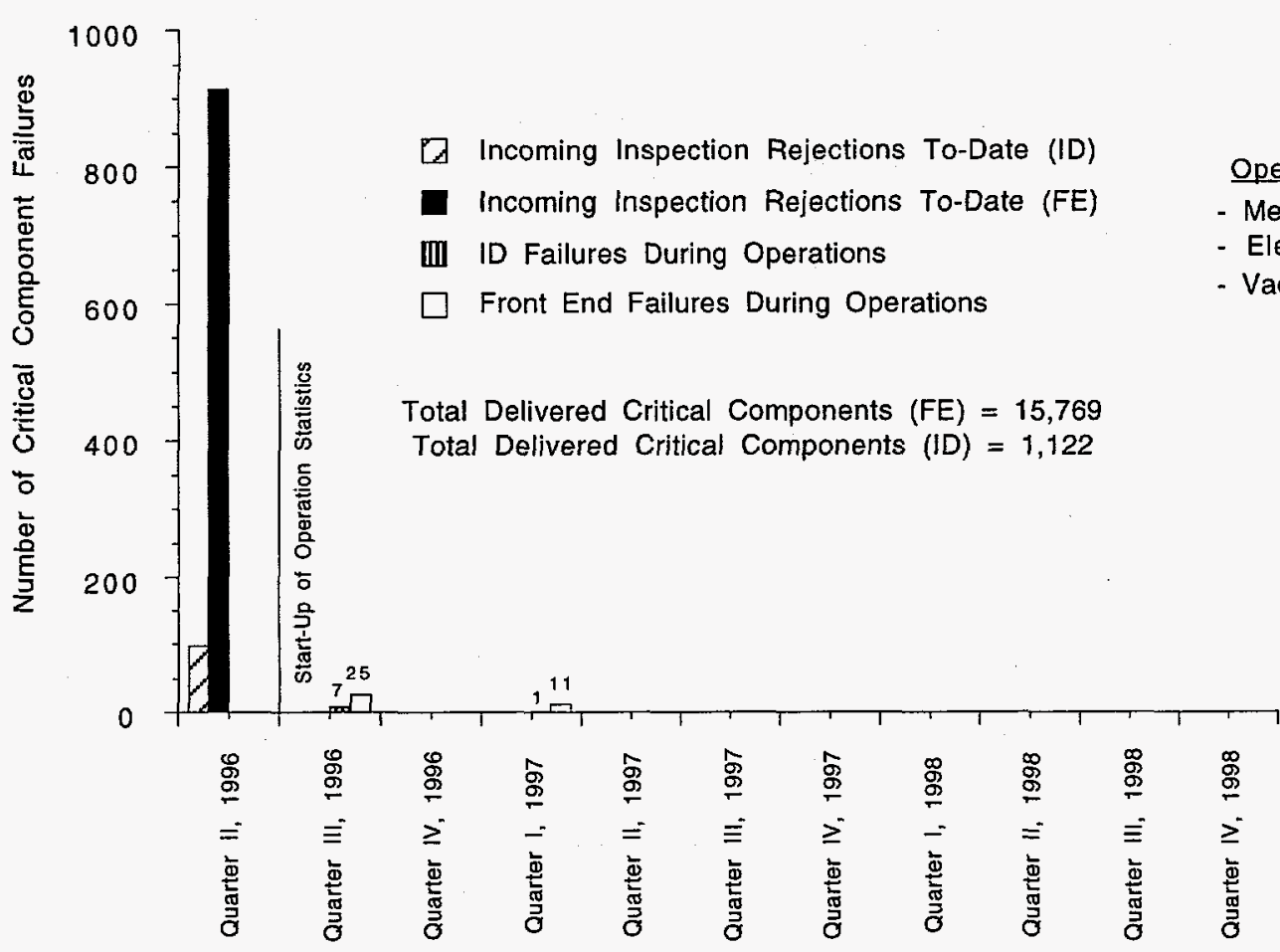

Fig. 2.12 Statistical data on component performance

contributed to 15.3 hours of downtime representing less than $1 \%$ of the total downtime. The XFD Operations contribution to $\mathrm{x}$-ray beam downtime during the operational cycles from quarter II, 1996, to quarter I, 1997, is presented graphically in Fig. 2.13.

2. The $x$-ray beam availability for all systems ranged from $62 \%$ (worst case) to $80 \%$ (best case).

3. It is still too early to detect any trends in the mean time to failure.

\subsection{User Operations Interface and Support}

\subsubsection{Beamline Commissioning Process}

The XFD has instituted a review process to ensure that the beamlines, which are constructed, installed, commissioned, and operated by the CATs, meet the APS facility safety and operational requirements. The process includes reviews through the design, installation, and commissioning phases of the beamlines. All the CATs go through a rigorous set of reviews at various stages of the development of their beamline designs, as 


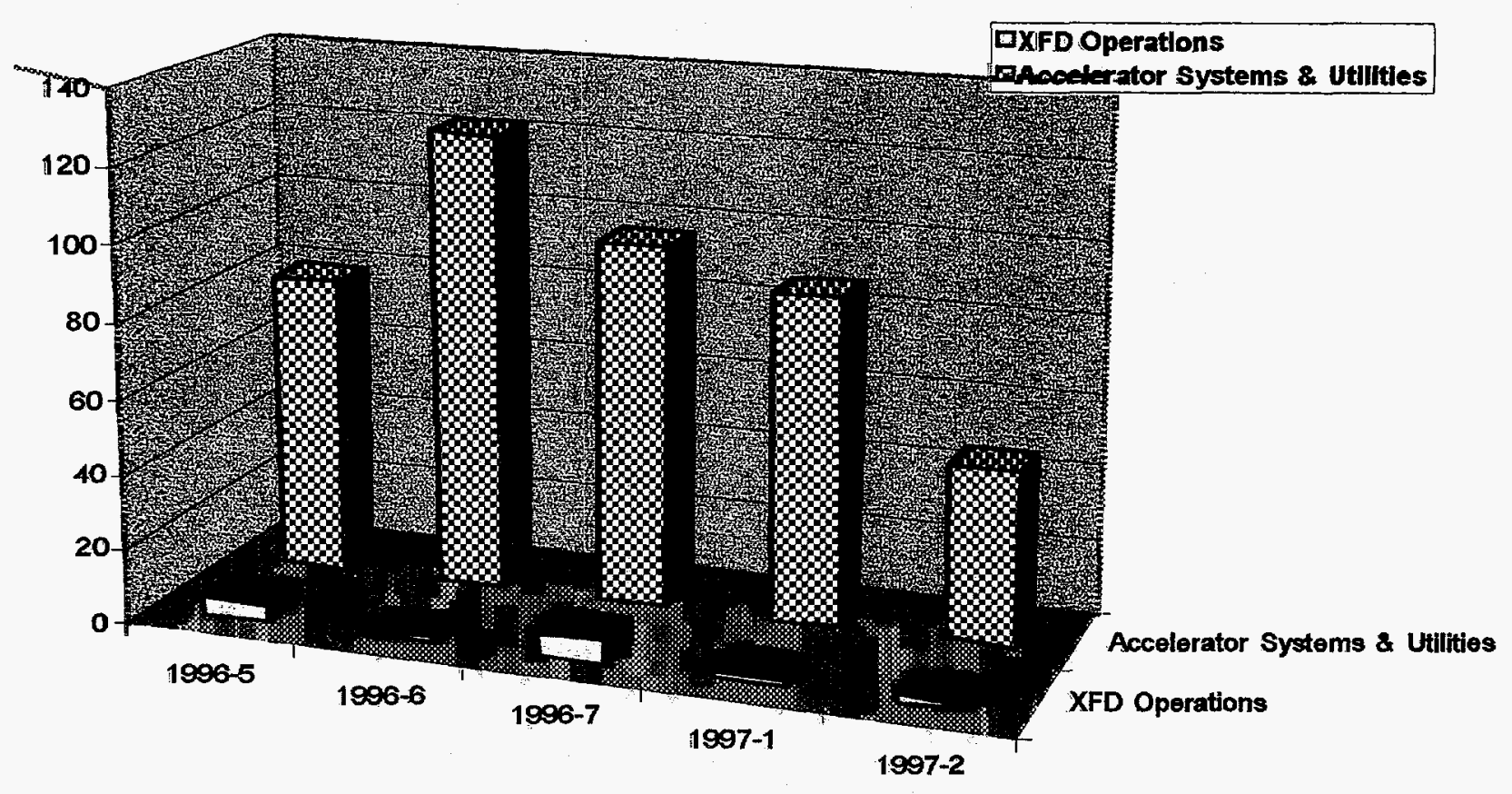

Fig. 2.13 XFD contribution to beam downtime

described in Chapter 3. Before the CATs begin installation of beamline components on the APS experiment hall floor, they have their installation plans approved by the APS as part of the CAT Management Plan. The plan identifies the activities to be carried out on the APS experiment hall floor, personnel involved in those activities, responsibilities of the personnel, hazards associated with the activities, and required training for the personnel. As the installation work proceeds, the CATs begin planning for the commissioning.

The XFD has the responsibility for ensuring that user commissioning activities comply with requirements stated in the Accelerator Safety Order, DOE 5480.25. The order requires that prior to the start of operations, a readiness review be conducted. The XFD has prepared a document entitled Advanced
Photon Source Experimental Beamline Commissioning Readiness Process, which addresses the process that the APS staff and the CAT personnel will use in commissioning the beamlines. The commissioning readiness review is one of the most critical phases in the beamline review process. Its objective is to ensure that the staged commissioning of the beamline can be performed in a safe and efficient manner. To this end, the process involves the following steps: (a) defining the various stages of beamline commissioning and the commissioning parameters leading to an operational configuration in each stage; (b) checkout of beamline components in each stage; (c) checkout of the required PSS and EPS in each stage; (d) defining the required managerial control systems and procedures and verifying readiness of personnel (both APS and CAT); (e) verifying assignment of responsibilities during the commissioning and 
verifying that personnel have received appropriate training for these responsibilities; (f) identifying the potential hazards and mitigation methods; and (g) addressing and resolving any interface issues between the CAT commissioning requirements and the facility. To support the validity of the process, the document provides a set of forms that have to be completed and validated by APS and CAT personnel.

The APS is willing to commission a beamline even if only segments are installed and ready for commissioning. On the other hand, if the CAT has installed a complete beamline, it is prudent that the commissioning be done in segments. Commissioning a segment of the beamline at a time spreads the commissioning burden over a longer period.

A typical staged commissioning scenario for an ID beamline is presented here as an illustration:

Stage 1: Commission the FOE (X-ID-A, where $\mathrm{X}=$ sector number). This involves commissioning of the ID, the FE, the empty enclosure (X-ID-A, normally the FOE) and the safety interlocks (PSS) required for the safe operation of this enclosure. Either a white-beam stop that is passively safe or a stop that is interlocked to the PSS to shut down the FE is included in the enclosure, depending on the commissioning envelope and the heat loads to be handled. The CAT may suggest the location of the beam dump to reflect a realistic operational configuration of the enclosure. The actual commissioning activity for this stage is described in a procedure; the primary focus here is on validating the shielding and the functionality of the safety interlocks. The XFD also performs photon beam diagnostics during this period, if necessary. The XFD has the prime responsibility for this commissioning stage.
Stage 2: Commission one or more of the instruments (filters, slits, monochromators, etc.) in the FOE (X-ID-A). The primary responsibility for this commissioning stage is with the CAT. When the instrument receives the first beam, XFD supports the commissioning and evaluates the radiation surveys performed by health physics technicians.

Stage 3: Repeat Stage 2, if more instruments are to be added to the FOE (X-ID-A).

Stage 4: Commission the next enclosure, an experiment station (X-ID-B), if it is ready to receive radiation. This phase is similar to Stage 1, and the primary responsibility is with XFD.

Stage 5: Commission instruments in the experiment station (X-ID-B). This is identical to the activities described above during Stages 2 and 3.

In general, additional stages of commissioning will be similar to Stages 4 and 5. There could be deviations from the sequence if the beamline is more complex or if the CAT desires to commission instruments in more than one enclosure in a single commissioning stage. It is also important to point out that in stages involving the commissioning of an enclosure (e.g., Stage 1 or 4 above), the commissioning is performed primarily by the XFD staff, while the instrument commissioning phases (such as Stages 2, 3, and 5 above) are performed mainly by CAT personnel under XFD oversight.

The initial planning of the commissioning stages is done by the CATs and approved by the APS as a part of the CAT beamline commissioning readiness review. The readiness review is conducted by a committee (the Beamline Commissioning Readiness Review 
Team, BCRRT), organized by XFD and including CAT representation. Prior to introduction of any beam into the beamline, the BCRRT conducts a visual inspection of the beamline and reviews the APS-conducted test of the PSS interlocks. The BCRRT also develops the beamline operation limits, which are based on the results of the shielding verification and defines the conditions under which the beamline may operate. These include, but are not limited to, the inclusion of hard stops on the $\mathrm{ID}$ gap, minimum supplemental shielding requirements, identification of beamline zones that may not be modified without BCRRT approval, etc. The beamline operation limits provide to the Floor Coordinator the necessary information to effectively oversee beamline operation.

The APS Experimental Beamline Commissioning Tutorial Workshop, held on May 16, 1995, described the entire process for commissioning of CAT beamlines. More than 60 representatives from all of the CATs attended the workshop, which was presented by XFD staff.

\subsubsection{User Controls}

The $\mathrm{x}$-ray beam is available for users during periods called User Operations. During User Operations, once the beam is stored in the storage ring with the correct orbit, the operators in the main control room enable the permit to open shutters. At this point, any CAT whose beamline is ready to operate can ask the APS Floor Coordinator to enable the beamline stations. Once the Floor Coordinator has enabled a station, the user is granted control for the FE and the appropriate beamline shutters. Currently the shutter opening and closing is accomplished from any of the PSS user panels located near each station. The PSS allows shutter operation only when all conditions are satisfied. A system for remote actuation of shutters is being implemented and will allow users to open shutters from additional control locations. When the stored beam is lost, the permit to open shutters is revoked by the storage-ring operators.

In the case of ID beamlines, the users have control of the ID gap during user operation. The control of the ID is given to the specific beamline once the beam has been stored and shutter permit is enabled. Upon beam loss, the ID control is taken over by storage-ring operators and all gaps are opened for injection. Currently the gaps are opened during injection to minimize possible radiation damage to the ID magnets. During mature operation, when all the injection variables have been stabilized, it is expected that injection will take place with all gaps closed. Once injection is complete, the gaps are returned to their last value before beam loss and control is turned over to users

\subsubsection{Operations Information Distribution}

A major effort is underway to distribute information to the users in a timely manner. Owing to the unified nature of the EPICS control system, thousands of items are monitored at all times. To avoid confusion, careful screening is performed and all relevant information needed for the users' operations is appropriately disseminated.

One of the methods being used is the World Wide Web. All relevant information about the storage-ring status, vacuum, ID status, FE status, and beamline status is available constantly with updates at one- to two-minute intervals. In addition, users can access data for any operating period during the past 
90 days in the form of a plot or raw data. A listing of the primary Web pages maintained by XFD is included in Appendix 3.

An EPICS gateway has been installed to provide the users with real-time data at data rates of $\sim 1 \mathrm{~Hz}$. This allows the users to access the data in real time for direct input into their data acquisition systems. A high-bandwidth optical fiber system is being implemented that will provide a direct communication link to the users' control systems at rates exceeding $100 \mathrm{~Hz}$.

A cable TV system with 14-channel capability is being implemented with TV screens located along the perimeter corridor of the experiment hall with expansion capability to the user beamlines and LOM areas. The information to be provided will include operation status, schedule updates, and other information that is pertinent to the user community. This will include in situ measurement of beam image and beam stability.

An on-line Web-based system has been implemented to report and track problems occurring with any XFD equipment. This allows the facility to monitor equipment performance, but also permits the user to do correlation studies with possible discrepancies in the collected data. During User Operation, when a problem is observed it is immediately recorded on the Web-based form. The person who responded to the problem in turn completes the Web-based trouble report on all actions taken. The system manager then reviews the trouble report and closes the report. Upon review by the system manager, feedback is given to the originator in the form of an e-mail.

An on-line Web-based system for work requests has also been implemented. No work can be performed by APS staff on any APS controlled system or on any beamline without a properly approved work request. A person planning to work on any system fills out an on-line work request form. If the work includes any components that may have an impact on user operation at a particular beamline, the Floor Coordinators, as well as CAT representatives, are notified of the pending work in that sector. In addition, the CATs at the neighboring beamlines are notified about pending work around their area. If a CAT has any problem with the proposed work schedule, the system manager works with the CAT and rearranges the schedule. Once the work is completed, the system manager closes the work request. It should be pointed out that this system also interacts with ASD personnel if the work requires ASD attention.

All work request forms and trouble reports can be reviewed by any of the users at any time. There are Web-based search engines to review these reports.

\subsubsection{User Operations Support}

The APS Floor Coordinators are members of the User Technical Interface Group and provide the day-to-day technical support for the APS users. In addition to their support role, the Floor Coordinators provide the primary APS oversight of beamline operations. The Floor Coordinators' offices are distributed around the experiment hall, with each Floor Coordinator assigned to the four sectors that are associated with a specific LOM. Floor Coordinators familiarize themselves with the operation of the beamlines within their areas of responsibility and have the authority to suspend operations if they feel that unsafe conditions may exist. Whenever the facility is operating or whenever beamlines 
are undergoing significant modification, a Floor Coordinator will be "on duty" representing the APS.

The principal responsibilities of the Floor Coordinators are as follows:

- Act as an interface/coordinator/ expediter between the APS users and the support services of ANL and the APS to facilitate user installation and operation activities. The Floor Coordinators help guide the user through obtaining the service and help to ensure that the work meets the user's specifications and fulfills the requirements of the APS. As users' needs for support from ANL have continued to grow, the Floor Coordinators have worked with the support organizations to tailor their services to meet these needs.

- Work with the APS CATs on the design, installation, and operation of beamline facilities to enhance the safety and the quality of the beamline installation and operations and to ensure that APS and ANL requirements are met. During the past year, a major activity of the Floor Coordinators has been to support installation of beamline utilities. The Floor Coordinators have processed the users' service orders and worked with the contractors and ANL to set up the tools that allow users to monitor the progress of their jobs. Another major activity during the past year has been the support provided to the beamline shielding validation process.

- Provide oversight, in the experiment hall on behalf of the APS, especially of safety aspects of the construction, installation, and operations of APS user facilities. Whenever $\mathrm{X}$-ray beams are being used in the experiment hall, a Floor Coordinator is on duty to oversee the beamline commissioning and operational activities. Also, the Floor Coordinators oversee the beamline configuration controls and manage the work permits for systems that are under configuration control.

- Work with other APS staff members on the development of user support facilities, such as the user LOM machine shops, the liquid-nitrogen supply tanks, and user-related policies and procedures.

During the past year, eight Floor Coordinators have been hired, and their number will continue to increase to support the growing number of beamlines and users. The Floor Coordinator team is being built with personnel who are experienced in a variety of different aspects of the construction and operations of research facilities.

\subsubsection{Radiation Shielding and Measurements}

\section{Shielding Design Criteria}

The APS is a DOE user facility and is subject to all DOE requirements concerning radiation safety. For DOE facilities, the primary document on radiation safety is the U.S. DOE Radiological Control Manual; Revision $1 .^{4}$

4 U.S. DOE Radiological Control Manual; Rev. 1. Primary Report Number: DOE/EH-O256T-Rev. 1. 
It is a general document that specifies the requirements for many aspects of radiation safety including radiological standards, conduct of work, radioactive materials, training, and record keeping. It is required that new DOE facilities be designed so that the annual integrated dose equivalent received by an individual is no more than 500 mrem.

With the assumption of a 2000-hour working year, this becomes a dose equivalent rate of $0.25 \mathrm{mrem} / \mathrm{hr}$. The value of $0.25 \mathrm{mrem} / \mathrm{hr}$ is the criterion used throughout the APS experiment hall to define the operational safety envelope. The beamline shielding, inclusive of the experiment stations, is designed to meet this criterion. The U.S. DOE Radiological Control Manual; Revision 1 also requires the establishment of an ALARA (As Low As Resonably Achievable) program to guide the design and operation of every radiationproducing facility. Implementation of the ALARA concept is also taken into account in beamline design, commissioning, and operation at the APS.

\section{Shielding Validation of the Experiment Stations}

The Experimental Facilities Division, in collaboration with Health Physics personnel from ANL's Environment, Safety and Health (ESH) Division, performs the shielding verification of all the experiment stations. The CATs are allowed to proceed with beamline commissioning activity only after the successful completion of the shielding verification. When a shielding deficiency is detected, the CAT is informed immediately, and activity on the beamline cannot proceed until the deficiency is mitigated.
The purpose of the shielding verification of the APS experiment stations is twofold:

- To confirm that the amount of lead used in the various panels is of sufficient thickness.

- To verify that the joints between panels, wall/floor and door/floor, etc., are radiation tight and do not allow significant radiation to leave the enclosure.

The shielding verification is done for bremsstrahlung, synchrotron radiation, and neutrons. The methodology of the shielding verification is to simulate a worst-case scattering scenario inside the station and to conduct a radiation survey outside the station. In order to do this, worst-case scatterers were designed for each type of radiation.

\section{Bremsstrahlung}

Bremsstrahlung produces an electromagnetic shower when it encounters a target. The photon converts to an electron-positron pair, which radiates and, in turn, produces fresh pairs; the number of particles increases exponentially with depth in the medium. This process continues until the particles are below the bremsstrahlung production threshold, i.e., the critical energy of the medium. Then the particles lose energy by ionization, and the shower tapers off. The maximum shower energy occurs at a shower depth of approximately $\ln \left(\mathrm{E}_{0} / \mathrm{E}_{\mathrm{c}}\right)$ in radiation length of the medium, where $E_{0}$ is the initial energy of the photons, and $E_{c}$ is the critical energy of the medium. Any medium of approximately this 
thickness simulates the worst-case scattering scenario for bremsstrahlung from the point of view of dose considerations downstream of the scatterer. Table 2.5 shows the depth at which the maximum shower energy occurs for some elements of interest. A smaller thickness of the medium will cause an incomplete shower development, and a larger thickness will attenuate the shower energy.

Because of coulomb scattering, the electromagnetic shower initiated by bremsstrahlung also spreads out laterally. The radial depth of the shower is determined by the radiation length of the medium and the angular deflection of the particle per radiation length at the critical energy. For all materials, this lateral spread of the shower is of the order of one Moliere unit, which is approximately equal to $R_{m}=21.1\left(X_{0} / E_{c}\right)$. Almost $90 \%$ of the shower development takes place within a radius of one Moliere unit. Therefore the ideal "worst scatterer" should be of a thickness corresponding to the shower maximum (approximately 5 to 6 radiation lengths) and with lateral dimensions of approximately one Moliere unit. Table 2.5 gives the dimensions of a worst-case scatterer for bremsstrahlung,

Table 2.5 Results of the "Worst Scatterer" Calculations for Bremsstrahlung

\begin{tabular}{|l|c|c|}
\hline $\begin{array}{c}\text { Scattering } \\
\text { Material }\end{array}$ & $\begin{array}{c}\text { Thickness }(\mathrm{cm}) \\
\mathrm{X}_{0}{ }^{*} \ln (\mathrm{E} / \mathrm{Ec})\end{array}$ & $\begin{array}{c}\text { Transverse } \\
\text { Dimensions } \\
\left(2 \mathrm{R}_{\mathrm{m}}\right)\end{array}$ \\
\hline Tungsten & $2.5 \mathrm{~cm}$ & $2.0 \mathrm{~cm}$ \\
\hline Lead & $3.8 \mathrm{~cm}$ & $3.2 \mathrm{~cm}$ \\
\hline Copper & $8.3 \mathrm{~cm}$ & $3.0 \mathrm{~cm}$ \\
\hline Iron & $10.0 \mathrm{~cm}$ & $3.2 \mathrm{~cm}$ \\
\hline Silicon & $47.7 \mathrm{~cm}$ & $9.2 \mathrm{~cm}$ \\
\hline Aluminum & $44.7 \mathrm{~cm}$ & $8.2 \mathrm{~cm}$ \\
\hline
\end{tabular}

one that can be used for the shielding verification.

The worst-case scatterer for bremsstrahlung is also a worst-case source and scatterer of secondary neutrons from bremsstrahlung.

\section{Synchrotron Radiation}

Scattering of synchrotron radiation from a target material is determined by three major factors:

- incoherent scattering (Compton) cross section

- photoabsorption cross section

- density of the material

The dimensions of the target for maximum scattering of synchrotron radiation must be optimized with all three factors taken into account. For example, high- $Z$ elements have a larger incoherent scattering cross section and also a larger photoabsorption cross section in the energy range of interest to us $(10-500 \mathrm{keV})$. Therefore, a thick target of high-Z material will have not only good scattering probability but also considerable self-absorption. Table 2.6 gives the photoabsorption cross section and the incoherent scattering cross section at $100 \mathrm{keV}$ for some materials of interest and their ratio. This ratio must be small for a good synchrotronradiation scattering material. Table 2.6 shows that a target of low- $Z$ material a few millimeters thick $(<10 \mathrm{~mm})$, like aluminum, is a good choice. 
Table 2.6 Photon Cross Sections for the Target Materials at $100 \mathrm{keV}$

\begin{tabular}{|l|c|c|c|}
\hline \multirow{2}{*}{ Material } & \multicolumn{2}{|c|}{$\begin{array}{c}\text { Photon Cross Section } \\
\left(\mathrm{cm}^{-1}\right)\end{array}$} & \multirow{2}{*}{$\begin{array}{c}\text { Ratio } \\
\left(\sigma_{\mathrm{pe}} / \sigma_{\text {Incoh }}\right)\end{array}$} \\
\cline { 2 - 3 } & $\begin{array}{c}\text { (photo- } \\
\text { electric) }\end{array}$ & (incoherent) & 0.006 \\
\hline Graphite & 0.0014 & 0.232 & 0.130 \\
\hline Aluminum & 0.049 & 0.375 & 1.525 \\
\hline Iron & 1.560 & 1.023 & 2.247 \\
\hline Copper & 2.540 & 1.129 & 40.300 \\
\hline Tungsten & 74.700 & 1.854 & 51.360 \\
\hline Lead & 58.300 & 1.135 & \\
\hline
\end{tabular}

Tungsten is used as the bremsstrahlung scatterer; it is placed upstream of the station so that the scattered shower subtends the maximum solid angle at the back wall of the experiment station. The locations selected for the synchrotron scatterers depend on the configuration of the experiment station. The beam is scattered simultaneously from two scatterers, placed at approximately $1 / 3$ and $2 / 3$ of the length of the station. This placement maximizes the radiation field inside the station. The radiation survey is carried out by appropriately calibrated survey equipment for bremsstrahlung, synchrotron radiation, and neutrons.

\section{Measurement of Gas Bremsstrahlung from the ID Beamlines}

Bremsstrahlung is produced in the APS storage ring when the particle beam interacts with the storage-ring components or with the residual gas molecules in the storage-ring vacuum. The interaction of the particles with the gas molecules occurs continually during storage-ring operation. Gas bremsstrahlung is important at the $\mathrm{ID}$ straight sections because the contributions from each interaction add up to produce a narrow monodirectional beam that travels down the beamlines. At the APS, with long storage-ring-beam straight paths (15.38 meters), gas bremsstrahlung in the ID beamlines can be significant.

The bremsstrahlung spectrum and the total energy radiated in a beamline are measured by a hermetic lead glass calorimeter. These measurements were carried out at the FOEs of the ID beamlines. The undulator was kept fully open to minimize the synchrotron radiation background. The calorimeter consists of 25 lead glass blocks, each $6 \mathrm{~cm} \times$ $6 \mathrm{~cm} \times 35 \mathrm{~cm}$ in size. Twenty-five phototubes connected to the lead glass blocks collected the signal. The bremsstrahlung spectrum and the total energy radiated were measured as a function of beam current $(20-100 \mathrm{~mA})$ at $7.0 \mathrm{GeV}$ particle energy for both electrons and positrons. The measurements were repeated at four runs at three different beamlines (12-ID, 10-ID, 13-ID). During data collection, six ion gauges continuously monitored the vacuum in the entire straight section.

The measured bremsstrahlung spectrum was fitted to a function that shows an approximate $1 / \mathrm{E}$ behavior. The gas bremsstrahlung rate from the APS undulator-beam straight path of $15.38 \mathrm{~m}$ is measured as $60 \pm 2 \mathrm{GeV} /$ $\mathrm{sec} / \mathrm{mA} / \mathrm{nT}$ Torr. This corresponds to a maximum dose equivalent rate of approximately $4.2 \times 10^{-4} \mathrm{~Sv} / \mathrm{hr} / \mathrm{nTorr} / \mathrm{mA}$. The analysis also shows that there is a significant bremsstrablung contribution from sources other than the residual gas molecules in the beam straight path in the storage ring. The maximum bremsstrahlung energy rate measured in a beamline is $369 \mathrm{GeV} / \mathrm{sec} / \mathrm{nTorr} / \mathrm{mA}$, which corresponds to a dose equivalent rate of $2.18 \times 10^{-3} \mathrm{~Sv} / \mathrm{hr} / \mathrm{nTorr} / \mathrm{mA}$. 


\section{Measurement of Radiation Dose Received by the IDs}

The radiation doses received by the magnetic structures of the insertion devices were found to be a few Mrads for each run. Thermal luminescent dosimeters (TLDs) are inadequate to measure this high dose. A program was initiated to use radiochromic films to measure these doses. Radiochromic films are freestanding films, 40-50 microns thick, of a solid-state solution (nylon). These change color when irradiated, and the dose rates are estimated from the optical density. The range of radiation dose these films can read varies from $10 \mathrm{krad}$ to $100 \mathrm{Mrad}$.

The radiochromic films were placed at various locations on each of the IDs before each storage-ring run. The films were read initially before being packed in aluminized mylar envelopes to shield them from the UV radiation. A computerized reader was installed to automate the optical-density reading process. After each storage-ring run, the films were collected and read. It was a challenging task to separate the synchrotron-radiation dose from the high-energy gamma dose arising from the particle beam loss at the transition piece between the storage-ring vacuum chamber and the ID vacuum chamber. For this purpose, a multilayer lead stack was designed with radiochromic films placed between intermittent layers. This develops the electromagnetic component of the dose by showering, while the synchrotron-radiation component is absorbed in the first few millimeters of lead. The initial results are very encouraging. These measurements have been repeated for the last several runs. The results are discussed in Chapter 4 (section 4.1). 

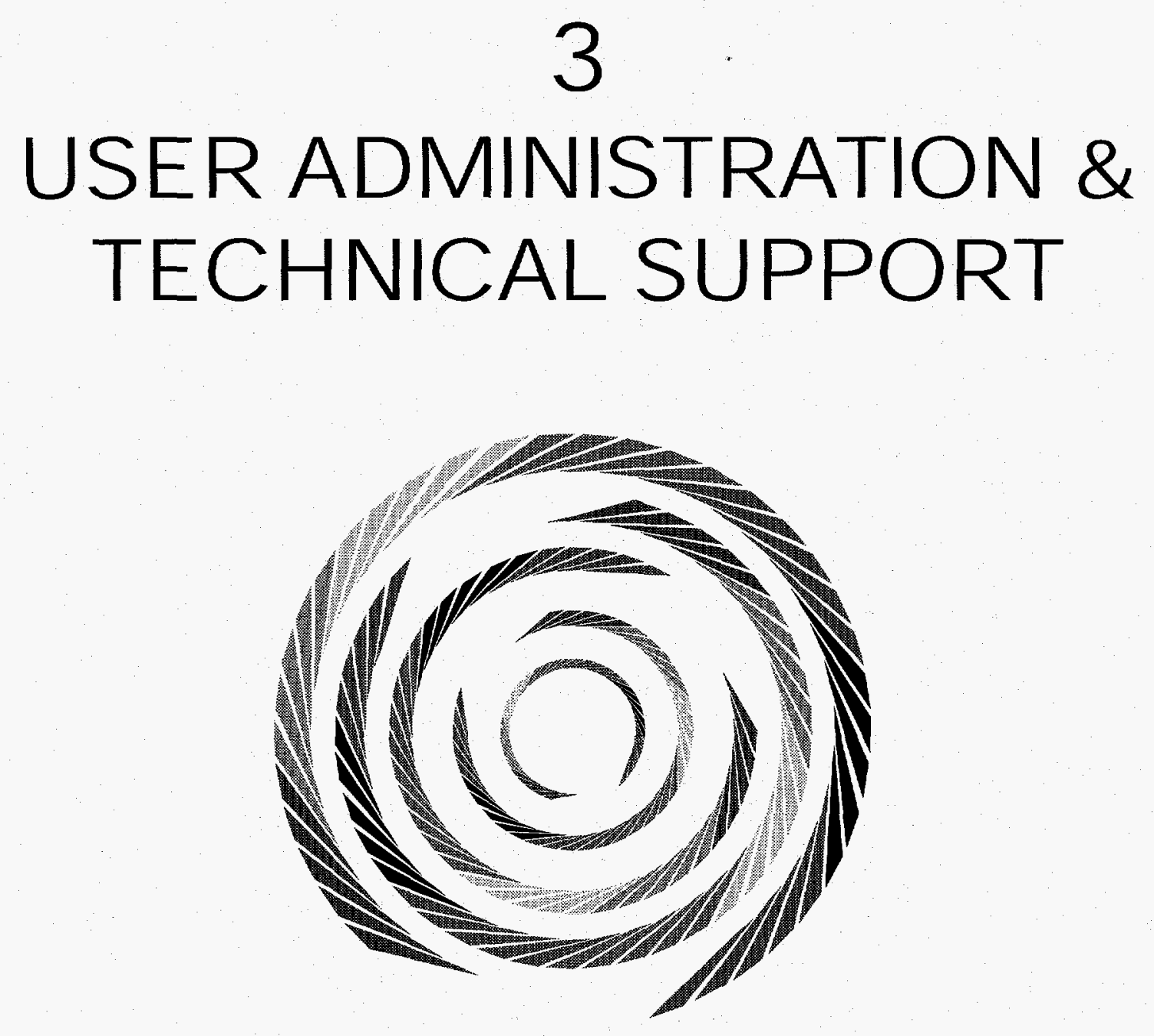


\subsection{User Administration}

\subsubsection{APS Users}

\section{Selection Process}

Users of the APS work either as members of Collaborative Access Teams (CATs) or as Independent Investigators.

Collaborative Access Teams. The CATs are selected by means of a comprehensive process developed in 1989. A prospective CAT submits a Letter of Intent (LOI), which is reviewed by the APS Program Evaluation Board (PEB), a six-member international scientific advisory body appointed by the Advanced Photon Source (see Table 3.1). If the LOI is approved, the prospective CAT is invited to submit a complete proposal. The proposal must include a description of the proposed scientific program, with emphasis on the need for the unique capabilities of the APS and the innovative nature of the research itself. The key personnel must be identified, and conceptual designs for both a bendingmagnet and an insertion-device beamline must be presented. Additionally, the proposal must contain a description of the funding strategy and an outline of the management structure, as well as any special requirements for utilities or other facility or policy issues. The scientific sections of the proposals are reviewed by external appointed Scientific Review Panels, which send their reviews to the PEB. The PEB then invites the group to make a formal presentation. When a proposal is approved, the prospective CAT must submit a detailed conceptual design report, and a comprehensive management plan for APS approval, and it must document the intent of its funding agencies or sponsoring institutions to provide sufficient funding to complete the beamlines as originally proposed. When these requirements have been satisfied, the APS signs a Memorandum of Understanding (MOU) with the CAT and assigns a sector location on the APS experiment hall floor.

Independent Investigators. CATs select Independent Investigators by means of proposal processes developed and managed by the CATs. Each CAT is required to make a minimum of $25 \%$ of its beam time available to Independent Investigators, beginning one year from the time a particular experiment station in its sector is declared operational.

\section{Current Status}

Collaborative Access Teams. To date, the APS has received 37 LOIs, of which 25 have been approved by the PEB. Twenty-two proposals have been received, 15 of which were approved. Two additional proposals are expected for review by the PEB in May 1997. The 15 approved proposals account for 20 sectors and (owing to the recent merger of two CATs) represent 14 CATs. Memorandums of Understanding have been signed for 19 of these sectors; as soon as sufficient funding is received for the remaining sector, another MOU will be signed. Together, these CATs comprise 828 principal investigators and support staff from 168 home institutions.

Appendix 4 summarizes the APS reviews and approvals completed to date for each of the 14 CATs in allocating 20 sectors.

Independent Investigators. At present, none of the CATs have experiment stations that have been operational for a year. Independent Investigator access plans are now being 
Table 3.1 Past and Current APS PEB Members

\begin{tabular}{|c|c|c|}
\hline Name & Affiliation & Term \\
\hline Jens Als-Nielsen & Københavns Universitet & $1989-1995$ \\
\hline $\begin{array}{l}\text { Howard Birnbaum } \\
\text { Current Chair }\end{array}$ & $\begin{array}{l}\text { University of Illinois, } \\
\text { Materials Research Laboratory }\end{array}$ & 1989-present \\
\hline $\begin{array}{l}\text { Roy Clarke } \\
\text { APSUO Representative }\end{array}$ & University of Michigan & $1993-1994$ \\
\hline $\begin{array}{l}\text { Stephen M. Durbin } \\
\text { APSUO Representative }\end{array}$ & Purdue University & 1991-1993 \\
\hline $\begin{array}{l}\text { Alan I. Goldman } \\
\text { APSUO Representative }\end{array}$ & Iowa State University & $1994-1995$ \\
\hline Wayne A. Hendrickson & Columbia University & 1989-present \\
\hline $\begin{array}{l}\text { Paul Horn } \\
\text { APSUO Representative }\end{array}$ & IBM & $1989-1991$ \\
\hline $\begin{array}{l}\text { Michael L. Knotek } \\
\text { Past Chair }\end{array}$ & Pacific Northwest Laboratory & 1989-1995 \\
\hline Denis B. McWhan & Brookhaven National Laboratory & 1995-present \\
\hline Roger Pynn & $\begin{array}{l}\text { LANSCE, } \\
\text { Los Alamos National Laboratory }\end{array}$ & 1989-present \\
\hline Jochen R. Schneider & HASYLAB & 1995-present \\
\hline $\begin{array}{l}\text { D. Mark Sutton } \\
\text { APSUO Representative }\end{array}$ & McGill University & $1995-1997$ \\
\hline
\end{tabular}

developed by the majority of the CATs; however, these plans have yet to be approved by the APS.

\subsubsection{User Administrative Functions}

The APS User Office serves as the initial point of contact for prospective and incoming APS users and handles the following administrative and support functions: user communication, user access and orientation, conference/ workshop planning and support, data management, and support of user advisory groups. Administrative functions handled by the XFD Division Office include user policy development, user agreement development, and user account management. 


\section{Communication}

Primary means of communication with users include periodic mass mailings either to the entire user community or to APS CAT members; periodic e-mail messages to the user community or selected subsets (such as APS User Organization Steering Committee members, APS Research Directorate members, APS users on site, etc.); a periodic newsletter, CAT Communicator, which is sent to all APS CAT members and other selected individuals; and periodic World Wide Web postings. Other forms of communication include conference and workshop reports, the APS User Guide and APS User Safety Guide, and various technical memos and technical bulletins. Additionally, an electronic newsletter is now being developed, and a User Activity report, describing user science at the APS, will be produced for the first time in 1997.

To date, the APS has produced 17 issues of CAT Communicator, 6 Conference Proceedings, 15 Technical Memos or workshop reports, and 26 Technical Bulletins. (See Appendix 5 for a bibliography.)

\section{User Access and Orientation}

Argonne National Laboratory is a controlled access facility; consequently, APS users must have gate passes or APS user badges to enter the Argonne campus. The APS experiment hall itself is protected by a Cardkey ${ }^{\mathrm{TM}}$ access system (which should be activated in 1997). When a user registers with the APS and completes the core training requirements (described in the section on "User Safety and Training"), he or she will be issued an APS user badge (printed on Cardkey ${ }^{\mathrm{TM}}$ stock) that enables him or her to enter both the Argonne campus and the APS experiment hall through Cardkey ${ }^{\mathrm{TM}}$-controlled doors in the laboratory/office modules. User badges can also be used for access after normal business hours and on weekends to the APS central laboratory/office building, where the stockroom and library are located.

\section{Conferences and Workshops}

Conferences and workshops are planned regularly for the APS user community. User meetings are held approximately every 18 months. The First Users Meeting for the APS took place on November 13-14, 1986; the Eighth Users Meeting for the APS was held on April 15-17, 1997. Appendix 6 lists the conferences and workshops held since 1990.

\section{Data Management}

Information about APS users is stored in a relational data-management system developed by the APS User Office. This database is currently set up in a client-server configuration and is used by a number of APS staff members (including User Office and Division Office staff, Floor Coordinators, and procurement and accounting personnel) and Argonne staff (Conference Services and Human Resources) needing access to current APS user information. The Directory module is used throughout the APS as an electronic "Rolodex" for user contact information and is accessible on a "read-only" basis to anyone with database client software. Additional modules include CAT Information, User Accounts, User Agreements, Training, Registration, Beamline Design Reviews, and Meeting Registrations. Each module has separate read/write access privileges and is 
appropriately password protected. Modules under development include Program Evaluation Board, APS Users Organization, and Research Directorate modules, as well as an Independent Investigator proposal module. Plans are now being made to transfer the Directory module to the APS World Wide Web intranet for wider access.

\section{Support of User Advisory Groups}

Administrative support is provided to three main user-related advisory groups, as well as several other smaller groups.

The six-member Program Evaluation Board (which was formerly called the Proposal Evaluation Board) meets at least annually to review new Letters of Intent and new proposals and to conduct annual progress reviews of existing CATs.

The 18-member APS Research Directorate (RD) includes APS senior management and the Director of each APS CAT. The quarterly meetings of this Directorate are chaired by Associate Laboratory Director David Moncton and facilitated by a CAT Director (on a rotating basis). The primary purpose of the meetings is to serve as a forum for the discussion of CAT scientific management issues. Development of policy is an expected outcome. APS staff support to the RD includes the APS User Program Administrator, the APS User Technical Interface, and the XFD Assistant Director for Policy and Planning.

The APS User Organization Steering Committee meets quarterly and serves as a support, advisory, and advocacy group for the APS. The Vice-Chair of the User Organization serves as the Scientific Program Chair for
User Meetings. The APS User Administrator serves as the primary APS liaison for this group.

\section{User Policies and Procedures}

The APS User Policies and Procedures is a comprehensive umbrella document being developed by XFD with input from other ANL organizations, the CAT Directors, the APSUO Steering Committee, and the APS Program Evaluation Board. Its purpose is to provide guidelines for all aspects of APS participation by both CATs and Independent Investigators and to clarify the roles of the APS staff and the various user advisory groups. The outline of the current version is shown in Appendix 7. As sections of the document are completed, they are distributed to the CAT Directors and posted on the Web.

One of the provisions of the MOUs that are signed by the APS and the CATs is a statement that the APS and the CAT will operate in accordance with the APS User Policies and Procedures.

\section{User Agreements}

To comply with the terms of the Prime Contract between DOE and The University of Chicago, Argonne National Laboratory (ANL) must sign formal User Agreements with the home institutions of all APS users. These agreements address a number of issues, including the general types of work users may do at the APS; payment for reimbursable expenses; intellectual property rights; liability and indemnification; ownership and disposition of property; requirements for approval of third-party contracts; and ANL's right to stop a user's work if safety rules are violated. In 
consultation with ANL's Legal Department, DOE, and the user community, XFD has developed the set of standard APS User Agreements that are now in use. XFD also manages the process of identifying the appropriate type of agreement for each institution and working with the institutional contacts to obtain signatures. As of this writing, APS User Agreements are in place with 58 institutions, including 28 universities and non-profit institutions, 17 industrial firms, 9 U.S. Government-funded labs, and 4 international institutions; another 14 institutions have received APS User Agreements for signature.

\section{User Accounts}

At the APS, as at all DOE User Facilities, users doing nonproprietary research (that is, research intended to lead to publication in the open literature) are not charged for machine time. However, DOE requires payment for machine time, on a full cost recovery basis, by users doing proprietary research; and all users must reimburse the facility for ancillary equipment, supplies, and services. The APS User Account system was developed and implemented by XFD, in collaboration with several other ANL organizations, to manage the reimbursement process. A given user institution may have any or all of the following types of APS User Accounts:

Construction accounts are used for certain categories of capital construction work performed by ANL employees or ANL subcontractors, such as utility installation along the beamlines and the buildout of office space inside the laboratory/office modules (LOMs).
Capital equipment accounts are used for beamline components (including radiation enclosures) purchased through or fabricated by XFD on behalf of a CAT for installation outside the shield wall.

Operating accounts are used for materials, supplies, and services. Examples include telephone service; items from the APS stockroom; Personnel Safety System installation; effort charges for employees hired by $\mathrm{XFD}$ and assigned to a CAT to perform scientific or technical work for the CAT; and space charges (i.e., building/utility/custodial charges associated with occupancy of LOMs).

Proprietary accounts are used for proprietary beam time.

As of this writing, 68 APS User Accounts are in place, serving 31 user institutions. XFD works directly with the users to establish these accounts, monitors the accounts with respect to dollar limits and expiration dates, and provides input to ANL's Accounting Department for the preparation of detailed invoices for all purchases.

We have been able to reduce APS users' beamline development costs considerably by including them under the umbrella of ANL's current "grandfathering" policy. By virtue of this policy, construction and capital equipment purchases by member institutions of existing CATs are exempt from ANL indirect charges until the end of FY 1997.

Advance payment is not required on any APS User Account that is funded with DOE money; also, as a result of intensive discussions between XFD staff and DOE-Chicago officials, advance payment is not required on operating accounts. 


\subsection{User Safety and Training}

At the APS, safety is a line management responsibility that is shared by the CATs. The following are the basic elements of the approach used by the APS and the CATs to create and sustain a safe working environment for APS users:

- The CATs incorporate appropriate engineered safeguards into their APS facilities.

- Each CAT conducts its activities at the APS in accordance with a written safety plan developed by the CAT and approved by the APS.

- Users receive appropriate safety training for their activities at the APS.

- The APS, ANL, and the CATs themselves perform safety oversight of user activities.

The first of these elements is discussed in "Beamline Designs" (section 3.3.1). The others are discussed below.

\subsubsection{CAT Safety Plans}

Each CAT is expected to develop, and maintain as a "living document," a safety plan that reflects the current makeup of the CAT's safety program as the CAT organizes itself and moves through the installation and commissioning phases and into the operations phase. The plans are intended to supplement the ANL Environment, Safety and Health (ESH) Manual, which is incorporated by reference, and relevant safety manuals of the
CAT member institutions. The preliminary safety plan (which is part of the written management plan that each prospective CAT submits as one of the prerequisites for obtaining a sector assignment) must describe the CAT's commitment to safety, the safety responsibilities assigned to specific individuals, and the reporting relationships of these named individuals within the CAT organization. Before beginning hands-on work at the APS, each CAT must expand this write-up into a comprehensive plan that describes the CAT's safety policies, organization, and management practices; identifies the specific hazards to which its users, and other individuals in or near its facilities, may be exposed; and describes how the CAT will control these hazards. The CAT safety plans must be approved by the Safety Plan Review Group, which is composed of XFD staff, before the CATs can begin the corresponding activities. Once a CAT's safety plan is approved for a particular phase, a fundamental safety responsibility of the CAT is to conduct its on-site activities in accordance with that plan.

The Experimental Facilities Division has assisted the CATs in the development of their safety plans by providing a model plan that can be tailored to reflect each CAT's activities and organizational structure. Some of the topics addressed in detail by the model plan are the distribution of safety functions within the CAT organization; chemical, electrical, and ionizing radiation hazards; power tools; personal protective equipment; construction areas; hazardous waste; sealed radioactive sources; hoisting and rigging; use of LOM shops; and work-area inspections. This model plan is a living document: the initial version, issued in October 1993, was followed by an updated version in August 1995 and supplements in May and June 1996 as needs for additional guidance were identified. 
A key feature of each CAT's safety plan is the assignment of specific day-to-day safety responsibilities to an on-site CAT member, usually called the CAT Safety Coordinator. Typically, this individual advises the CAT Director on safety-related matters; works with on-site CAT management to ensure that the CAT's activities are consistent with its safety plan; conducts sector-specific and some taskspecific training (see "User Safety Training" below); and serves as the CAT's primary contact with the APS on safety issues.

As of this writing, 14 CATs (covering 19 sectors) have APS-approved safety plans in place (see Appendix 4). Several of these CATs are currently expanding the scope of their plans in anticipation of their formal entry into the operations phase. In particular, they are filling in the details of their procedures for safety reviews of proposed experiments. For all CATs, this process will incorporate the use of a standard APS Experiment Safety Approval Form, which was adapted from the comparable form used at the National Synchrotron Light Source (NSLS). This form is completed in part by the experimenter, who describes the materials and equipment to be used, the known hazards, and the ways in which these hazards will be mitigated; and in part by the CAT Director or designee, who reviews the information, makes recommendations as needed, and ultimately signs off to indicate approval. The form must be posted at the beamline for the duration of the experiment. A Web-based system for completion and approval of the form is currently under testing.

\subsubsection{User Safety Training}

The responsibility for APS user safety training is shared by the APS and the CATs. This training falls into three broad categories:
"Core" training is required for all APS users and is administered by the APS User Office. As of March 10, 1997, 316 APS users have completed the core training program, which consists of the following elements:

APS User Orientation: This orientation, developed and updated as needed by XFD staff, is now being delivered to newly arrived APS users in a computer-based training (CBT) format; the topics covered include the role of the CAT safety plan, site alarms, emergency exits, use of 911 , dosimeters, configuration control, management of hazardous chemicals, experiment safety review, and others. Additional safety information is provided by the APS User Guide and the pocket-sized APS User Safety Guide, both prepared by XFD staff; these booklets are part of the user registration package and are also posted on the Web. The CBT itself will be available via the Web in the future. All incoming users sign a statement confirming that they have read and understood both the APS User Guide and the User Orientation and will follow the guidelines given there.

General Employee Radiation Training (GERT): The GERT training is a general APS requirement consistent with DOE policy. APS users who do not have a current GERT card from another DOE facility must pass a computer-based GERT exam. They may prepare for the exam by taking a CBT course at the APS or via the Web, or by reading a hard-copy study guide, which is part of the user registration package.

Generic Personnel Safety System (PSS) training: APS users view a videotaped introduction to the operation of the PSS.

Sector-specific training is also required for all APS users; it is administered by the CATs as a 
face-to-face orientation/demonstration. To facilitate this training, XFD has provided a model checklist of topics to be covered; each CAT may modify the list as necessary. The training focuses on communicating specific information needed to implement the CAT's safety plan; examples include locations of utility shutoffs, chemical storage locations and practices, procedures for obtaining and transporting liquid nitrogen, and beamlinespecific operation of the PSS. The CAT signs off on the checklist and sends a copy to the APS User Office to verify that each new user who will be working under its auspices has completed this training. As of March 10, 1997, the User Office has received signed-off "sector orientation records" for 97 APS users.

Only APS users who have completed both core and sector-specific training are given Cardkey access to the APS.

Task-specific training is the third element of APS user training. The CATs identify taskspecific training needs for their personnel and users in accordance with their CAT safety plans. Qualified CAT staff members may perform some of this training themselves; two examples are training users to operate the hoists in the experiment stations and orienting experienced machine tool users to the CAT's LOM machine shop. Many other task-specific training needs are met, in whole or in part, through courses offered by ANL's ESH Division; the XFD ES\&H Coordinator's office handles user enrollments in these courses. XFD supports the course selection process both by one-on-one consultation with users and by maintaining an XFD-developed computer program that matches available courses and certifications to planned activities. XFD has also worked closely with the ESH Division's Training Section (ESH-TR) to tailor courses and course requirements to APS users' needs.
To date, 24 different ESH-TR courses have counted APS users among their graduates. Some examples follow, along with the numbers of APS users who have completed each course to date:

ESH-119, Pressure Safety Orientation

ESH-145, Cryogenic Safety (16)

ESH-195, Personal Protective Equipment

ESH-574, Chemical Waste Generator (18)

\subsubsection{User Safety Oversight}

The responsibility for user safety oversight, like the responsibility for safety training, is shared by the APS and the CATs. The APS Floor Coordinators perform informal day-today safety oversight of user activities; XFD also has the lead role in formal user safety oversight, with two programs currently in place:

Reviews of CAT safety plans: As mentioned earlier, an XFD committee reviews the CAT safety plans to ensure that they are adequate for the upcoming phase of sector development and operation. The outcome of a typical review is an acceptance letter with detailed recommendations for improving the plan as the CAT implements the activities described therein.

Inspections of CAT-occupied areas: Periodic walkthroughs are conducted by the XFD ES\&H Coordinator, with invited participation by safety specialists from the Fire Protection, Industrial Hygiene, Health Physics, and 
Safety Engineering Sections of the ANL ESH Division. XFD then provides the CATs with written recommendations for addressing any concerns that are identified.

In addition, a procedure for XFD oversight of the CAT-managed experiment safety review process is currently under development.

To take advantage of the CATs' experience in managing their own safety programs at the APS, XFD has initiated the formation of three Safety Oversight Committees within which the CATs will conduct reciprocal assessments of each other's safety programs. Each CAT has been asked to name a representative to one of these committees, also known as "mutual safety assessment groups"; each CAT will then be reviewed by the others in its group on a rotating basis. Alternatively, a CAT may organize its own Safety Oversight Committee consisting of safety experts from its member institutions or ANL; the selection of members for such independent committees is subject to APS approval.

The CAT Safety Oversight Committees may adopt a set of model assessment criteria that have been provided by XFD, or they may develop their own criteria and obtain XFD approval. Each CAT will be reviewed at least annually by its fellow committee members and will receive a written report (which will be copied to XFD) identifying action items and a schedule for completing these actions. The committees will also be encouraged to make recommendations to the APS for enhanced safety support.

To date, 13 CATs (accounting for 16 sectors) have signed on to participate in mutual safety assessment groups; XFD is currently working with one of the three groups to "pilot" the assessment process.

\subsection{User Technical Support}

\subsubsection{Beamline Designs}

\section{Overview}

The CATs are responsible for the design of their beamlines and their associated experimental facilities. The APS supports these efforts through the development of standardized designs of many beamline components, working through a design review process to ensure that the plans meet applicable standards, and providing incidental design engineering for conventional construction. Standard designs are available to the synchrotron community through the APS Design Exchange (DX), which is described below. The formal review process extends from the conceptual design through preliminary and final beamline designs. The review process is described in detail in the following sections.

In the first step in a beamline design review, as part of the proposal process, the CAT develops a Conceptual Design for the planned beamline. The Conceptual Design is reviewed by an Instrumentation Feasibility Study Panel of the Program Evaluation Board. An approved Conceptual Design is one of the prerequisites to assigning a specific APS sector to a CAT for the construction and operation of beamlines. Once the proposal has been approved and a sector has been assigned to a CAT, the CAT will proceed to develop detailed beamline designs.

The APS/XFD has created a Beamline Review Committee (BRC) to review the design and operation of each beamline. It is composed of APS scientists and engineers who are familiar 
with beamline technical requirements and is chaired by the group leader of the User Technical Interface Group. Advisors from other areas of ANL are added as issues are identified for which adequate expertise does not exist within the committee.

Beamlines are reviewed to ensure that their designs (1) are consistent with the proposed scientific program and (2) satisfy all APS, $\mathrm{ANL}$, and DOE requirements for safe operation. The documents developed for the review are kept on file in the APS User Office for future reference. In addition, at each review stage, drawings of the beamline and its components are filed in the DX, through which current electronic or paper copies of beamline layouts can be accessed as needed. The review process is intended to identify issues that must be resolved before beamline installation or commissioning begins.

The BRC reviews the beamline at several stages: (1) Preliminary Design Review; (2) Final Design Review; and (3) whenever significant modifications are to be made that may affect the safety aspects of the beamline, such as shielding, additional beamline sections, etc. Appendix 4 summarizes the status of these reviews.

\section{Preliminary Design Review}

The preliminary design of the beamline represents completion of approximately $30 \%$ of the design effort for each of the beamline components. This level of design permits the CAT to develop cost estimates for the construction of the beamline, as well as a realistic timeline for completion of the construction tasks. Following guidelines provided by the APS, the CAT prepares a Preliminary Beamline Design Report (PDR) for APS review. The PDR must include the following elements:

\section{Beamline layout}

- Layout of the beamline within the sector, showing components and support equipment

- Life Safety Code compliant egress aisles

- Some indication of the plans for survey and alignment of the beamline components

\section{Component designs}

- Appropriate specification of components that are not APS-developed standard components

- Assurance of compliance with APS policies, such as the APS vacuum policy

- Description of the optical and shielding apertures with ray-tracing analysis and the existence of a reasonable safety margin for white-beam components

\section{Management issues}

- Demonstration of a Work Breakdown Structure (WBS) with preliminary costs and schedules

\section{Special operating requirements}

- Identification of any special requirements and an evaluation of 
compatibility with the installed conventional facilities

\section{Preliminary safety analysis}

- Analysis of the shielding design for compliance with APS shielding standards

- Definition of the proposed modes of beamline operation, together with the expected requirements of the PSS

- Description of the beamline EPS and an assurance of compliance with the APS policy on white-beam beamline components

- An ozone mitigation plan with a preliminary analysis of ozone production within the beamline

- Identification of and preliminary mitigation plans for program-specific hazards, (hazardous gases, radioactive materials, etc.)

\section{Final Design Review}

The next phase of the APS beamline review process focuses on the Final Beamline Design Report (FDR), which is submitted when approximately $90 \%$ of the total design effort has been completed. APS approval of the CAT's designs described in the FDR is required prior to installation of beamline components in the APS experiment hall. Items that have a long lead time for design or procurement may be reviewed separately from the remainder of the beamline, but enough information must be provided so that reviewers can understand the context in which these components are to be used. Those components that are part of the APS standard component list are not reviewed for their individual performance; however, the components are reviewed in the general scheme of the total beamline performance.

The review of the PDR focused on the layout of the beamline as a whole and at a level that would permit the beamline components to be designed independently of each other. The FDR review focuses on aspects of safety, scheduling, required APS support, and updating the information provided in the PDR. The topics to be addressed in the FDR are as follows:

\section{Layout}

- Identification of changes from PDR layout

- Survey plan, including expected APS survey and alignment support

- Update of the ray traces provided in the PDR

\section{Component designs}

- Component final designs

- Assurance of compliance with the APS vacuum policy

Schedule, cost, \& WBS

- Installation schedule with indications of the expected APS craft support

- Survey and alignment schedule 
- Update of the WBS-based schedule given in the PDR

Safety

- Final PSS requirements

- EPS logic and interface requirements

- Description of final shielding design

- Identification of chemical, electrical, fire, and other hazards that impact beamline design, and means to be used for mitigation.

- Identification of program-specific hazards (high powered lasers, biohazards, etc.)

\section{Special operating requirements}

- Identification of special conventionalfacilities requirements

\subsubsection{Beamline Design Exchange}

\section{Overview}

At the APS, all the ID front ends are of one standard type and all the BM front ends are of a second standard type. Likewise, all the common components of the beamlines themselves have been standardized (Kuzay, 1992; Shu et al., 1995), hence the name "APS standard components." The standard front-end and beamline component designs, technical specifications, and statements of work for procurement are all housed in a sophisticated electronic depository called the APS Design Exchange (DX), a part of the Beamline
Engineering Group. The choice of standard components and their designs (which were prepared by XFD staff) have been reviewed by an independent committee of experts external to XFD.

The purpose of the DX is to distribute APS beamline component drawings and related technical information to the APS user community. This information is available on the Internet (or World Wide Web) to individuals with current passwords. However, comprehensive information has been provided with open access to explain to interested parties the operational features and access to the DX. Special care has been taken to make the DX Web site user friendly. General access to the DX on the Web is at the URL (uniform resource locator): $<\mathrm{http}: / /$ dxchange.aps1.anl.gov/>. Access to DX drawing libraries is password controlled to assure quality control, security, and intellectual property rights.

The majority of APS users (CATs) have chosen to use APS standard components, or somewhat modified forms of them to suit their specific needs, in their beamline designs. Hence the DX has become a major user support facility.

In addition to being an electronic copy depository, the DX has also been the source for creating both the logical drawing numbering system (universally adopted and used at the Advanced Photon Source Project) and the standard component naming conventions for cataloging and indexing these diverse components in a systematic way. It contains sophisticated search engines and electronic data storage and backup systems.

Table 3.2 lists the holdings contained in the DX. 
Table 3.2 DX Holdings in the Electronic Depository ${ }^{1}$

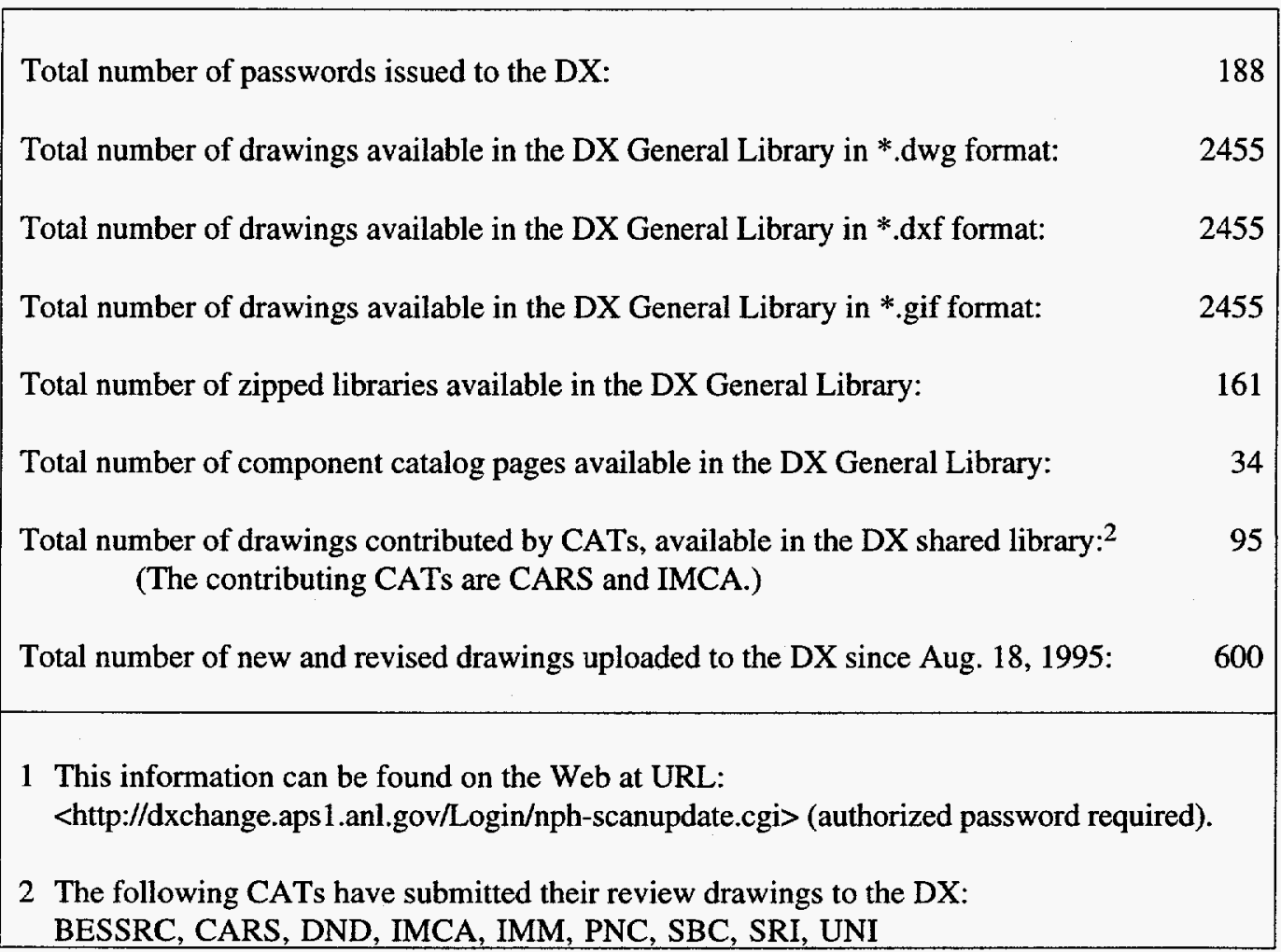

\section{Usage Statistics}

To date, more than 9,000 logins to the DX have been recorded, and more than 190,000 files have been downloaded.

\section{Hardware and Software}

Both the hardware and software for the DX have been developed in a systematic way since 1991. In 1993, the APS DX was initiated as a Web server to allow sharing of APS-designed standard components with CAT members around the world. The DX was the first Web server at the APS Project.

In late 1994, the role of the DX was broadened to include acting as a local server for XFD engineering and design personnel. In addition, special accounts are set up for the CATs to allow direct uploading to their individual shared or private libraries.

In January 1996, an Exabyte EXB-10h Autochanger was purchased. The EXB-10h is a 14 GB per tape autochanger with 10 tape slots. A Legato Networker was the choice for a backup utility. Currently, we have the capability of handling a total of 45 clients on different platforms.

\section{Current Activities}

In recent months, the DX activities have shifted to accommodate new requirements of the users. Less time is spent uploading new designs to the libraries, and more hours are 
spent assisting CATs planning to install APSdesigned standard beamline components. Many questions directed to the DX office address engineering issues, manufacturing techniques, and the interfacing of drive systems, encoders, and safety switches, as well as document and source information. We believe that our support to the CATs substantially reduces the learning curves for individuals who are responsible for installing beamline components at the APS.

Another beneficial spin-off from the DX is the relatively effortless development of an electronic data storage system. This new system permits us to centralize our entire file system of computer-created drawings. When completed, it will also function as our official archive for electronic files with practical quality assurance and search tools in place. This work is done on a limited part-time basis with available in-house resources.

\subsubsection{X-ray Optics Metrology and Fabrication}

\section{The APS Metrology Laboratory}

\section{Introduction}

Over 50 major mirrors are expected to be installed and used on the APS beamlines. These will be used for harmonic rejection, focusing, power filtering, or beamline branching. Before being shipped, mirrors are often evaluated by the vendor, in terms of figure and finish (i.e., slope error and roughness), as part of their quality-assurance process. However, there is a need for an independent evaluation of the optical surfaces of mirrors as part of the acceptance criteria before they are installed and commissioned on the beamline; the metrology laboratory at the APS has been built to fulfill this task. In the following sections, a basic description of the metrology instruments is provided together with a discussion of the surface quality of mirrors measured to date (also see Bresloff \& Mills, 1996, and Assoufid \& Mills, 1997). Finally, some future developments are outlined.

\section{The Metrology Laboratory Environment}

The laboratory is located in a class 10,000 cleanroom in the APS experiment hall. Mirror handling requires special cleanroom garment and shoe covers, and access to cleanrooms is restricted when measurements are performed.

To ensure repeatability and accuracy of the instruments, the room temperature stability is controlled to better than $+/-0.5{ }^{\circ} \mathrm{C}$ and is regularly monitored. All instruments are mounted on air tables to minimize the effect of vibrations, and they have the capability of handling large and heavy optics up $2 \mathrm{~m}$ in length and $90 \mathrm{~kg}$ in weight.

\section{The Metrology Laboratory Instruments}

The laboratory has three different instruments to evaluate optical surfaces over a range of spatial periods from a few microns up to 2 meters. These instruments are:

Long Trace Profiler (LTP). The LTP is an instrument designed to measure slope error and curvature of optical surfaces up to $2 \mathrm{~m}$ long. It has a sensitivity of $0.1 \mu \mathrm{rad}$ in slope and $0.5 \mathrm{~nm}$ in height and a reproducibility of better than $0.5 \mu \mathrm{rad}$ rms. Because of its 
speed, it offers a rapid and accurate means of evaluating the performance of a mirror bending mechanism. A recent adaptation of the LTP allows it to measure a surface profile of a mirror in either the horizontal or vertical direction. Measurement in the horizontal direction is often desirable because it eliminates gravitationally induced figure distortion. Figure 3.1 shows a photograph of the LTP system with the high heat load mirror for the 2-ID beamline being evaluated in the horizontal position.

TOPO Surface Profiler. The TOPO is a microscope-based instrument that uses visiblelight $(630.3 \mathrm{~nm})$ interferometry to measure surface roughness on the order of an angstrom. The optical head of the TOPO profiler can use either a $3 \mathrm{D}$ or $2 \mathrm{D}$ detector and is currently equipped with three objectives: $1.5 \mathrm{x}, 5 \mathrm{x}$, and $40 \mathrm{x}$. The $3 \mathrm{D}$ detector is used when a profile of a surface area (of a few $\mathrm{mm}^{2}$ depending on the objective) is needed with a minimum resolvable height of $3 \AA$. The $2 \mathrm{D}$ detector obtains a line scan with a resolution of $1 \AA$ along a line up to a few millimeters long, depending on the objective (e.g., $2.05 \mathrm{~mm}$ for a $5 \mathrm{x}$ objective).

WYKO 6000 Figure Interferometer. The WYKO-6000 measures the flatness of optical surfaces interferometrically by a technique similar to that of the TOPO. It is used for characterizing optical surfaces up to 6" in diameter (or large optics at grazing incidence angles).

All these instruments are currently operational. Various modifications of the basic systems are being developed for ease of operation and to improve handling of large optics.

To enhance the capability of the metrology laboratory, a fourth instrument, an atomic force microscope (AFM), is scheduled to be installed and commissioned beginning in April 1997. The AFM will be used, for example, to measure surface roughness, evaluate diffraction gratings, and measure thin-film step height. This instrument will be a valuable tool for better understanding surface topography on the atomic scale and relating it to light scattering. In addition, the results can be used to improve methods for making optical surfaces.

\section{Surface Quality of Some Mirrors Measured to Date}

In the past two years, more than ten major beamline mirrors, some up to $1.5 \mathrm{~m}$ long, as well as a variety of smaller optics, have been evaluated in the APS metrology laboratory. Most of these mirrors will be used on the APS beamlines and have been designed and procured by APS users, including members of SBC-CAT, SRI-CAT (Yun et al., 1996; Khounsary \& Yun, 1996; Lai et al., 1996; Randall et al., 1995; and McNulty et al., 1996), and DND-CAT. Two mirrors have been designed and are destined for other facilities in the USA, namely CHESS at Cornell University, and the Center for Advanced Microstructure and Devices (CAMD) at Louisiana State University. Surface microroughnesses ranging from 1 to $10 \AA$ and slope errors ranging from 1 to 4 microradians have been characterized and were found to be consistent with vendors' measurements. To avoid bias, data from the vendors were obtained only after all metrology measurements were completed and analyzed. Appendix 8 summarizes the results for mirrors to be used on the APS beamlines. To date, most of the mirror substrates have been made of either silicon or Zerodur; the others have been made of Glidcop and float glass. Most 


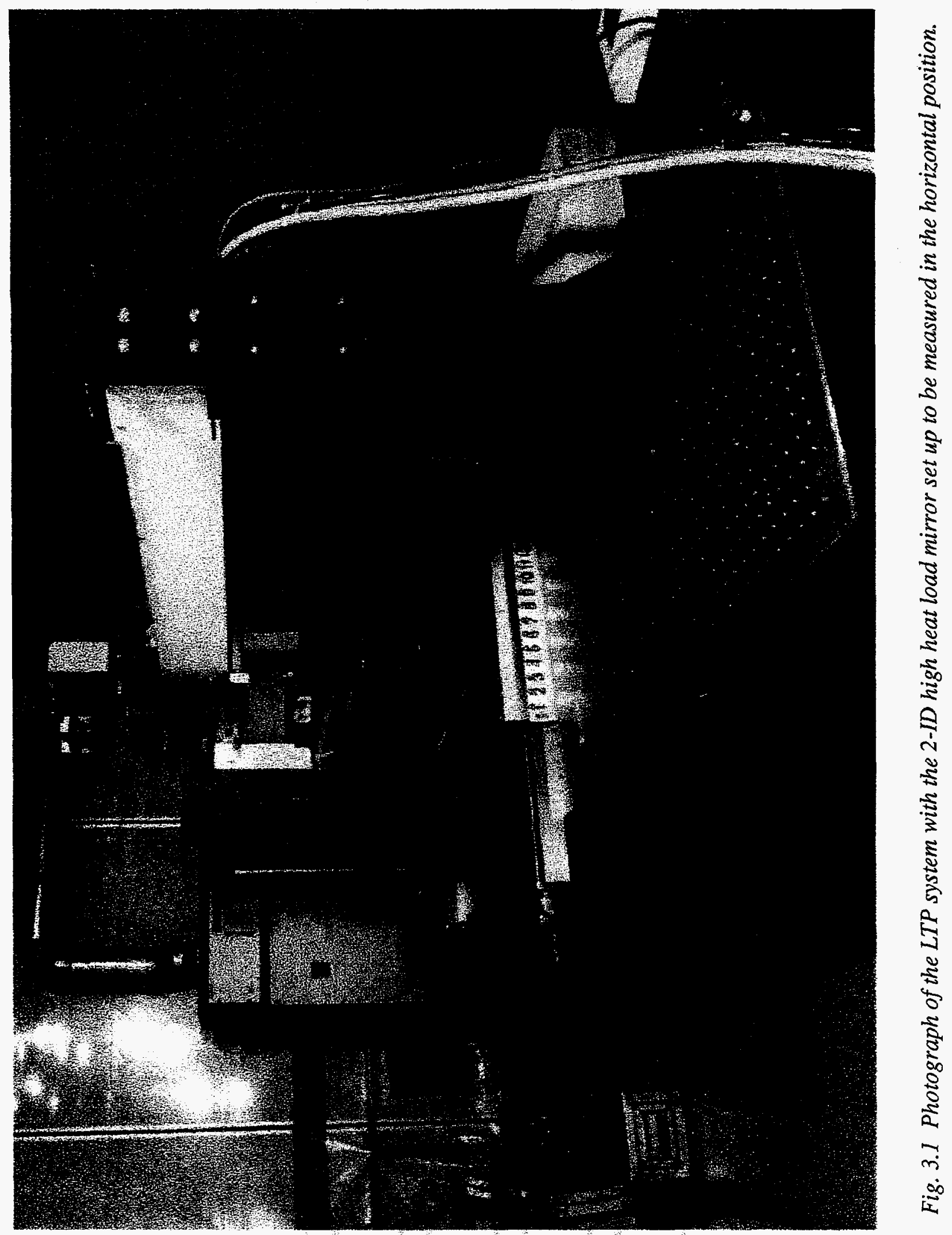


surfaces have at least one reflective coating; platinum and rhodium are typical reflecting materials. Figures $3.2 \mathrm{a}$ and $3.2 \mathrm{~b}$ show a typical output from the LTP. The results are for the 2-ID high heat load mirror (see Appendix 8), which is the first long mirror to be evaluated at the metrology laboratory. This mirror has been installed on Sector 2 and has performed well. Figure 3.2a shows the slope error profile (red curve) along with the corresponding height profile (blue curve) obtained by averaging five scans on the mirror center line. Figure $3.2 \mathrm{~b}$ gives the power spectral density of the derived average height profile. The power spectral density is useful for computing the rms statistics over a selected spatial frequency bandwidth. This allows one, for example, to separate "mid-frequency ripple" from overall surface figure.

\section{Coating Facilities}

To help develop reflective optical elements as well as experimental samples for all the APS users, we have established three coating facilities in recent years: (a) a $1.5 \mathrm{~m}$ sputter deposition facility, (b) a small sputter deposition facility, and (c) an evaporation facility. These facilities are briefly described below.

\section{Facility Descriptions}

$1.5 \mathrm{~m}$ Sputter Deposition Facility. This facility, shown in Figure 3.3, is located in the deposition lab, on the experiment hall floor next to Sector 1. The deposition lab is in a class 10,000 clean room with a gowning room entrance. The $1.5 \mathrm{~m}$ sputter deposition facility, also referred to as the large deposition system, consists of four large vacuum (a)

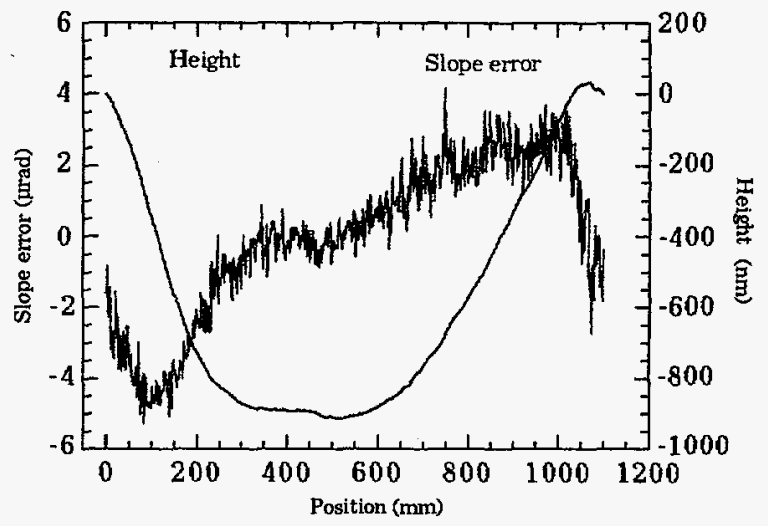

(b)

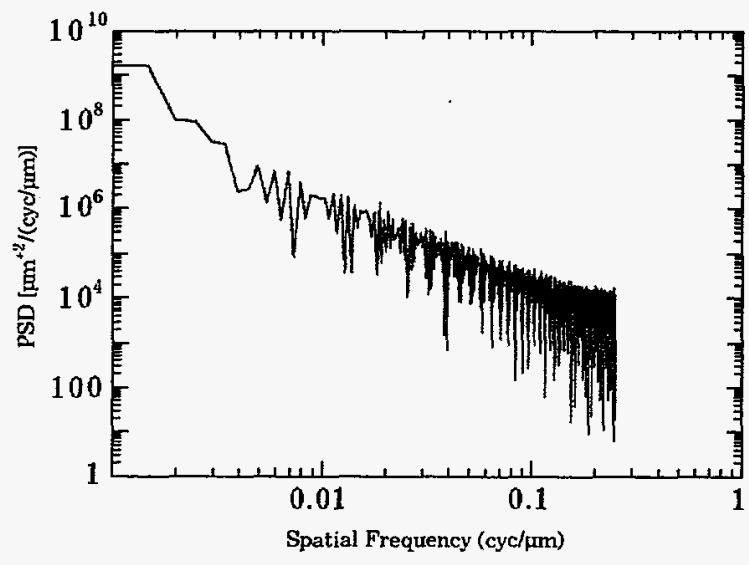

Fig. 3.2 Example of the LTP output. Shown are results for the high heat load MIP mirror for beamline 2-ID: (a) slope error and the corresponding height profile obtained by averaging 5 scans along the mirror center line, (b) power spectral density of the derived average height profile. The mirror is $1.2 \mathrm{~m}$ long and has a slope error of $2.2 \mu \mathrm{rad} \mathrm{rms}$. The corresponding rms height is $210 \mathrm{~nm}$, over a $1100 \mathrm{~mm}$ aperture.

chambers, each 16" in diameter and 66" long. The first chamber next to the clean hood is a load lock chamber isolated from the other three chambers by a computer-controlled gate valve. Three CTI model CT- 8 cryopumps and an Alcatel ADP 81 dry pump provide a base pressure of $<5 \times 10^{-8}$ Torr for the system. 

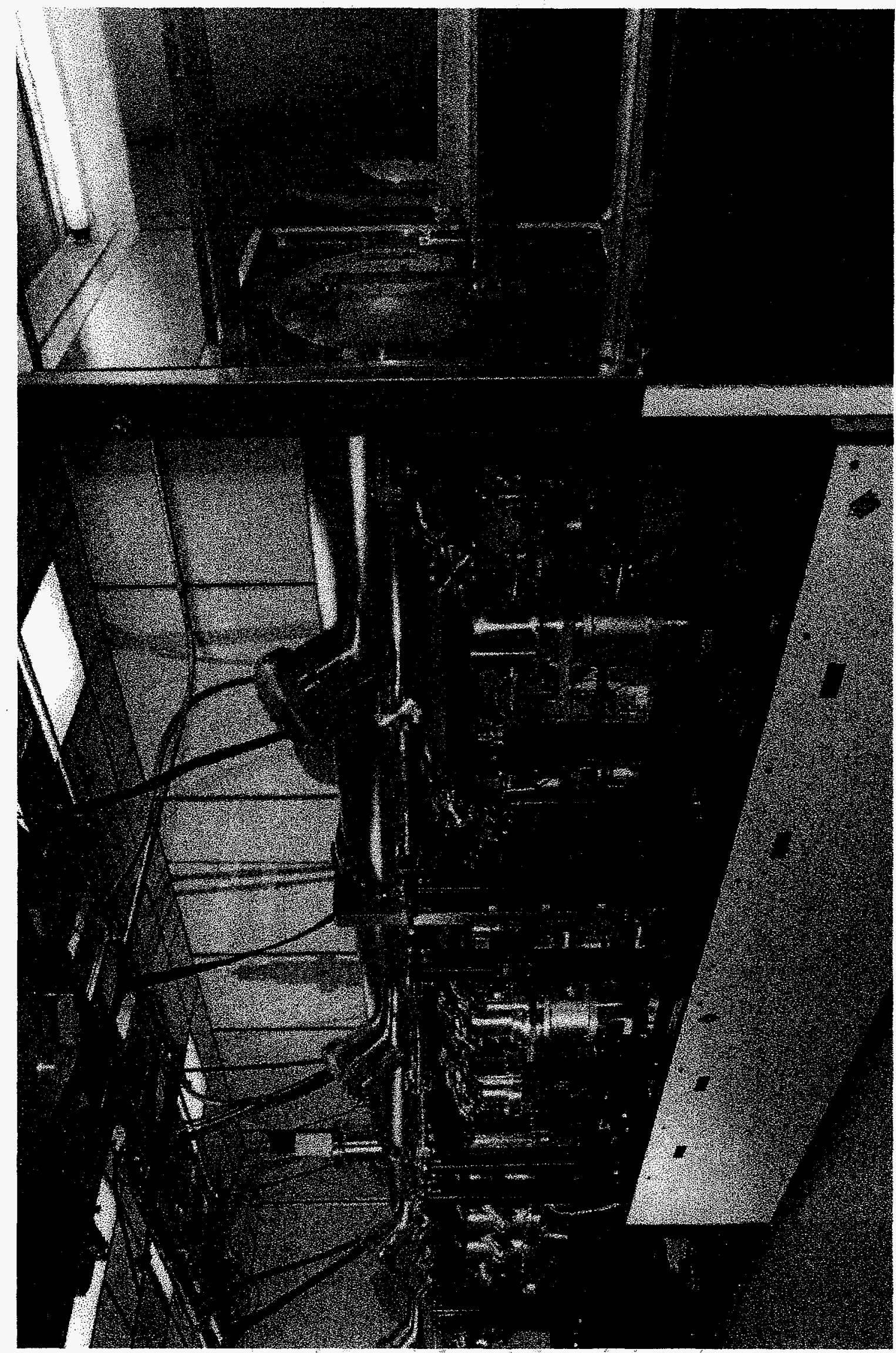

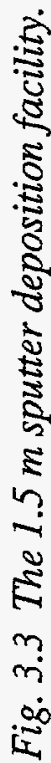


Mirror substrates as large as $150 \mathrm{~cm}$ long, $20 \mathrm{~cm}$ wide, and $14 \mathrm{~cm}$ high can be loaded into a substrate carrier inside the vacuum system using guard rails. The substrate can be outgassed in the load lock chamber using a UV lamp. The substrate-carrier transport assembly is driven by a stepper-motor. The full length of a $1.5 \mathrm{~m}$ substrate can be coated using the transport assembly.

The third vacuum chamber is the deposition chamber, where four ports on the bottom along the chamber axis are designed to house the sputter cathodes. Currently two 3" diameter magnetron sputtering guns compatible with both DC and RF operations are deployed. The coating is deposited in an upward direction with the substrate facing down. Argon pressure during the deposition is regulated by a MKS model 250 process controller, with the cryopump throttled. A coating uniformity of better than $\pm 5 \%$ over a 4" width has been achieved using a ratecontrolling aperture over the target source and the linear motion of the substrate. Similar uniformity over an 8 " width can be obtained by using two specially arranged guns.

Small Sputter Deposition System. This system has two 3" diameter DC magnetron sputter guns, located at the bottom of a 9.5" OD, 4' long vacuum chamber. The vacuum chamber is pumped by a turbomolecular pump and has a base pressure in the $10^{-7}$ Torr range. The system is designed to handle small substrates up to 4 " long and less than 0.5 " thick. The substrate-carrier transport assembly has a travel distance of $\sim 3$ feet. A uniformity of better than $\pm 5 \%$ can be obtained by using shaped apertures over the targets and the linear motion of the substrate. The argon pressure during deposition is regulated by using a throttled valve on the pumping station and a MKS process controller. The system is computer controlled and capable of making both single and multilayer coatings.

Evaporation Facility. This facility was created from an existing surface analysis facility, which is equipped with a Fisons Instruments CLAM 2 electron energy analyzer, an electron gun, a surface-cleaning sputter gun, and a residual gas analyzer. It is an ultrahigh vacuum system with a base pressure of $\sim 5 \times$ $10^{-11}$ Torr. Small samples (up to $0.875^{\prime \prime} \times$ 0.625 ") can be loaded to a heating/cooling stage on a XYZ manipulator through a load lock system. Ports at a lower level (compared to the analyzer level) on the vacuum chamber are available and can be used for thin-film deposition.

Miniature e-beam evaporators and precisiontemperature-controlled thermal evaporators were invented and made in-house for physical vapor deposition of thin/ultrathin films. At present, two evaporators are placed $\sim 20^{\circ}$ apart so that alloy thin films can be made via codeposition. A quartz-crystal thickness monitor is installed to monitor the evaporation rate before deposition. A third evaporator is installed in another vacuum port so that a protective coating can be deposited on the sample. The composition and uniformity of alloy films can be analyzed in situ using Auger electron spectroscopy. This system is suitable for MBE-type sample preparation.

\section{Current Activities}

With these three facilities, we are capable of fabricating virtually any kind of physically deposited thin film/multilayer for the APS users. To date, we have made over 100 depositions for the users and have accumulated experience on various thin 
film/multilayer systems. There are five major applications that require our deposition system: (1) hard x-ray mirrors, (2) soft $\mathrm{x}$-ray mirrors, (3) multilayer focusing mirrors, (4) coatings for $\mathrm{x}$-ray lithography testing, and (5) other thin-film/ multilayer samples for scientific research.

Common substrates for $\mathrm{x}$-ray mirrors include float glass, fused silica, silicon, Glidcop, etc. Coating materials are typically $\mathrm{Au}, \mathrm{Pt}, \mathrm{Rh}, \mathrm{W}$, $\mathrm{Ni}, \mathrm{Cr}, \mathrm{Ti}$, etc. Float glass used as a substrate has the advantage of a very low cost and reasonably good flatness. When it is coated with a glue layer of $\mathrm{Cr}$ or $\mathrm{Ti}$, the subsequent coating of metals, such as $\mathrm{Au}$ and $\mathrm{Pt}$, has good adhesion. $\mathrm{Cr}$ (Ti) can form covalent bonds to the glass by breaking the O-Si bond, and metallic bonds to other metals. Satisfactory results have been obtained for a batch of $\mathrm{Au} / \mathrm{Cr}$-coated float glass mirrors that we made for CARS-CAT. These mirrors are used for microfocusing of hard $\mathrm{x}$-rays. Using these mirrors, CARS-CAT has successfully focused a $60 \mathrm{kV} \mathrm{x}$-ray beam into a $6 \times 8 \mu \mathrm{m}^{2}$ spot. The gold coating remains intact after being subjected to hard x-rays, with no coloring, no clustering, and no cracking. A 56" long Au/Ti-coated mirror on float glass has also been fabricated for CAMD. It will be used as a soft $\mathrm{x}$-ray mirror. Successful cleaning methods have been demonstrated, which ensure a flat coating comparable to the substrate flatness.

Multilayer mirrors mostly utilize a high $\mathrm{Z} /$ low $\mathrm{Z}$ configuration. Common combinations are $\mathrm{W} / \mathrm{Si}, \mathrm{W} / \mathrm{C}$, and $\mathrm{W} / \mathrm{BC}_{4}$. These multilayers require a good thickness control and a stable deposition rate. For example, Prof. S. Durbin from Purdue has requested a W/C multilayer of 100 pairs of $W$ and $C$ with individual $W$ and $C$ layer thicknesses of $1.5 \mathrm{~nm}$ or less. A test multilayer of this kind has been fabricated recently on a silicon substrate. Other multilayers include $\mathrm{Ni} / \mathrm{C}, \mathrm{Ni} / \mathrm{Al}, \mathrm{Pt} / \mathrm{Al}, \mathrm{W} / \mathrm{Al}$, etc. Most of them are still in an experimental stage. We expect that the need for multilayer mirrors will grow substantially as more and more CATs start beamline commissioning.

Metal coatings are also used in $\mathrm{x}$-ray lithography experiments. In $x$-ray lithography, one needs a conducting layer on the silicon wafer to put on the photoresist layer. This conducting layer is later used as a base for electroplating, after which a pattern is formed through $\mathrm{x}$-ray lithography. To date the most common conducting layer we have used is $\mathrm{Au}$. We have grown $\mathrm{Au} / \mathrm{Ti}, \mathrm{Au} / \mathrm{Cr}, \mathrm{Ti}$, etc., on silicon and $\mathrm{Be}$ wafers. The $\mathrm{Si}$ wafer substrates worked very well. Currently we are experimenting on the metal/Be system.

Other coating applications include protective coatings, laser-mirror coatings, and other coatings for some special experiments. For example, by making special masks, we could make thin film/multilayers with specified shape and dimensions, which are very useful for some experimental studies.

On the evaporation front, we are growing $\mathrm{Fe}^{57} \mathrm{Sn}^{119}$ alloy films. These isotopes are very expensive, and it is not practical to use sputter deposition. Test samples have been made on glass and $\mathrm{Cr}$ /glass substrates. They are then coated in situ with $\mathrm{Si}$ as a protective layer. Final samples will be made on $\mathrm{Cr}$ coated fused silica substrates.

In summary, we have built basic coating facilities and started serving the APS community. We are continuing to improve our capabilities to meet the ever-increasing demand from our users. 


\section{Fabrication Laboratory}

The fabrication laboratory manufactures crystal elements needed for $\mathrm{x}$-ray beamlines at the APS. The lab is supplied with the following equipment:

Crystal Cutter (Meyer-Burger TS121). This CNC-controlled saw accommodates diamond blades up to 16 " diameter and can move a crystal along three mutually orthogonal directions with the travels up to $500 \mathrm{~mm}(\mathrm{X})$, $320 \mathrm{~mm}(\mathrm{Y})$, and $160 \mathrm{~mm}(\mathrm{Z})$. Additionally, the object (crystal) can be mounted on a rotary table and/or on a large sine bar.

STRASBOUGH 6DF-1. This lapper/ polisher is set up for coarse lapping of optical components (up to 12" dia). Slurries containing lapping compounds with grains of 50 and then 9 micrometers are used.

HYPREZ (ENGIS Corp. IL). This lapper/ polisher is for fine lapping of optical components (up to 12" dia.) with slurries containing 6 micrometer lapping grains.

STRASBOUGH 6DF-1. This lapper/ polisher is set up for final polishing of optical components (up to 12" dia.) with slurries containing 3 to 0.25 micrometer polishing grains.

Two ovens are available. The first oven is used for attaching (waxing or glueing) crystals to substrates/holders used during fabrication operations. The second one can be employed for annealing processes.

Two chemical hoods (lengths 8' and 6') are available for etching. (They are equipped with a special drain system.) In practice, one hood is predominantly utilized for etching, the other for work with solvents.

A crystal direct bonding setup consisting of a small laminar hood, wafer spinner, and fresh deionized water production line is used for experiments on silicon-to-silicon direct bonding. Wafers of up to 4" diameter can be placed on the spinner.

During the last year, the fabrication laboratory has manufactured 47 optical elements for use by various CATs in monochromators, analyzers, and interferometers.

\section{X-Ray Laboratory}

The objective of the $\mathrm{x}$-ray laboratory is to support operation of the fabrication laboratory and activities of other XFD groups that make use of conventional x-ray generators. The lab is supplied with the following equipment:

Rigaku X-ray Generator. This Rigaku x-ray generator produces $\mathrm{X}$-rays in conventional tubes (typical targets, $\mathrm{Cu}$ and $\mathrm{Mo}$; maximum power about $2 \mathrm{~kW}$ ). Two horizontal beamlines (point focus) are available. The beam emerging from the right port is collimated and employed for orientation of ingots or precut crystal pieces. A single-axis, manually operated diffractometer (Huber 424 goniometer) is used for crystal and detector rotations about the common vertical axis. Samples tested with this crystal orienter are usually fixed to holders that can be mounted on the crystal cutter table. The orienter is used frequently.

The beam from the left port is used for work with a double-axis diffractometer. The diffractometer is a commercial instrument 
made by Blake Industries, Inc. Only precise rotation of the sample table is automated and controlled by computer via EPICS. After reflection from a monochromator, the $\mathrm{x}$-ray beam typically has a $2 \times 2 \mathrm{~mm}$ footprint and is predominantly used for taking rocking curves. In the past, the instrument was very often employed for local testing of samples. It was also used for testing interferometers and area detectors.

Spellman $X$-ray Generator. X-rays are produced in conventional tubes (typical targets, $\mathrm{Cu}$ and $\mathrm{Mo}$; maximum power about $2 \mathrm{~kW}$ ). Two horizontal beamlines (point focus) are available. The beam from the left port can be utilized in an $8^{\prime} \times 9^{\prime} \times 8^{\prime}$ enclosure attached to the left side of the generator tower shielding. The enclosure can be entered through two sliding doors and can accommodate large size equipment. In the past, this beam was predominantly used for testing different kinds of prototype high heat load monochromator setups that were later installed at the APS ring.

The beam from the right port is shared by two types of experiments. A Laue camera may be installed just next to the beam port, and a pattern of backscattered reflections from a crystal can be examined. When the Laue camera is removed, the primary beam can be transported through a long vacuum tube to a separate enclosure. This additional chamber is primarily used for double-crystal reflection experiments requiring highly collimated primary beams.

Rigaku X-ray Generator. This second Rigaku x-ray generator is a rotating-anodetype generator with a maximum power of $18 \mathrm{~kW}$. Targets made of copper or molybdenum can be mounted. Two horizontal beamlines (point focus) are available. The beam from the right port is employed for work with a triple-axis diffractometer. The diffractometer is a mixture of purchased commercial units made by Blake Industries, Inc. (monochromator enclosure) and Huber (Two Circle Goniometer 422 and 511.1 Eulerian Cradle), and custom elements (coupling between the monochromator unit and the Huber goniometer, air pads lifting the goniometer, and the analyzer subassembly). This diffractometer was recently upgraded and is now routinely used for reflectivity measurements (so far, for checking the quality of samples later employed for experiments at the APS).

The primary beam emerging from the left port is transported in a long vacuum tube to another enclosure that surrounds a double-axis $\mathrm{X}$-ray diffractometer called the Topo Test Unit (TTU). This station was designed and built in-house for topographic testing of $\mathrm{x}$-ray optics elements. The distance between the source and the diffractometer axis 1 is about $2 \mathrm{~m}$. The primary beam-transport tube ends about $180 \mathrm{~mm}$ upstream from axis 1 (there is space available for long monochromators) and serves as a base for an entry slit system and an additional lead enclosure surrounding the first crystal.

The diffractometer is mounted on a special base composed of two separate plates that can be moved independently along two common, exactly parallel, precise rails. The so-called right base plate supports the first crystal table, while the rest of the diffractometer stays on the left base plate. The objective of the design was to build a machine capable of testing (in some steps) samples of front-face size up to $300 \mathrm{~mm} \times 90 \mathrm{~mm}$. With highly asymmetric cut monochromator crystals, a typical footprint of the monochromatic beam is $80 \mathrm{~mm} \times$ $90 \mathrm{~mm}$. The take-off (two theta) angle for the monochromatic beam can be manually adjusted in the range from 0 to 120 degrees. 
The distance between axes 1 and 2 can be fixed in the range $560+/-64 \mathrm{~mm}$.

The axis 2 assembly forms an independent single-axis diffractometer that can be slightly lifted up by the air pad. It is equipped with coarse and fine theta rotations. Motor resolution for the fine rotation is 0.00008 arcsec, which results in a smooth motion. In practice, steps of 0.1 arcsec are sufficient for rocking curve measurements.

Crystals mounted on axes 1 and 2 can be translated along and rotated about the reciprocal vector directions and tilted about horizontal axes normal to the translation directions.

Two Bicron scintillation detectors are used for monitoring intensities reflected from monochromator and sample crystals. They can be translated in the directions normal to the respective beams. Detector 2 has an entrance window that is 5 " in diameter, which is sufficient to accept photons from about 4" diameter samples at a theta Bragg angle close to 45 degrees. To date, images of Bragg reflecting samples have been registered on photographic materials. A film cassette must be inserted manually.

Most movements of the instrument segments are motorized and automated under EPICS software control. Also, rocking curve measurements and searches for the optimal tilt angle for the sample are computer controlled.

The TTU is used predominantly for testing prototype monochromators and single crystals to be used at the APS. The tests consist of measuring rocking curves in double-crystal geometry and taking crystal images, i.e., taking topograms. The TTU was also used for testing an interferometer, multilayer structures, and crystals for a customer unrelated to the APS.

High heat load monochromator testing has been performed in a few stages. Typically, a monochromator is first tested "free-standing" and then mounted into a holder for use with synchrotron radiation. The mounted crystal is again tested on the TTU. Usually the first mount introduces strains, and mounting screws have to be readjusted. Testing is repeated (sometimes a few times) until strains are removed.

During the past year, the TTU has been used to test 48 optical elements for various CATs.

\subsubsection{Beamline Controls and Data Acquisition}

The Experimental Facilities Division has undertaken the development of standard beamline software (and related electronic hardware) to support scientific users of the APS, in collaboration with and on behalf of APS CAT developers. The objectives of this undertaking are to maximize the quality of the user software that APS developers as a group can produce; to set minimum standards for quality and ease of use, on which all APS users can depend; and to ease the migration of users and experiments from one APS beamline to another. The expectations are that we will address needs common to many APS users and CAT developers, and that CAT developers will concentrate on needs unique to their beamlines and users.

The degree to which the objectives are met depends in part on the willingness and ability of CAT developers to implement and extend XFD-developed software. Developers representing all APS CATs agreed early on to base 
their beamline software on EPICS, where feasible, and to collaborate in its development. This agreement served principally to authorize XFD's early commitment to developing EPICS-based beamline software. Most of the current CAT developers are implementing XFD software on their beamlines or are working toward this end.

In return, XFD provides many forms of technical support to ensure that CAT developers have access to the tools and information required to apply and extend our software effectively: videotaped classes on EPICS development; workshops on beamline software; loaner development systems (VME crate, processor, licenses, etc.); technical services, such as PROM programming, help with initial software installation, hardware and software troubleshooting, and system/ network-administration help; Web-based documentation of beamline software, electronic hardware, wiring standards, etc., for developers; online documentation of beamline software for users (see Fig. 3.4), and a documentation kit (in progress) with which CAT developers can extend our user documentation to include their own products; coordination of volume hardware specification and purchases by the CATs; procurement and distribution of VxWorks (the real-time operating system underneath EPICS) licenses; distribution of EPICS software and of EPICSbased beamline software; and telephone and e-mail support.

In addition to developing, collecting, documenting, maintaining, and distributing standard beamline software, XFD also implements beamline software for SRI-CAT and maintains SRI-CAT's file server, computer network, and workstations. This arrangement helps to ensure that the software we produce meets users' needs and is field tested before it gets distributed to other CATs.
(It also means that XFD and SRI-CAT scientists normally perform experiments with software that is under active development.)

The beamline software developed by XFD is based on EPICS—a toolkit for building distributed control systems-which is the product of a large international collaboration of developers supporting accelerators (e.g., APS, CEBAF, DESY), synchrotron-radiation beamlines (principally at APS), telescopes (e.g., Gemini, Keck), and large detectors (e.g., the Gammasphere, and RHIC's Solenoidal Tracker). In addition to developing software useable by other members of the EPICS collaboration, XFD developed and maintains the software-distribution mechanism for the collaboration. In these ways, we help to "pay" for the technical support burden imposed by APS and CAT developers on other EPICS collaboration members and for new developments in EPICS of which APS users are the principal beneficiaries.

Because synchrotron-radiation users and developers are a minority in the EPICS collaboration and because some of our users' needs are atypical of the collaboration as a whole, it is essential that we find ways to influence the direction in which EPICS development proceeds. Recently, a technique that allows limited reprogramming of a live control system, for which we had been lobbying for several years, became a standard part of EPICS. (The technique was developed largely by APS ASD with some funding and much beta-testing support from XFD. Developers at Los Alamos also made substantial contributions.) Currently, we are working to keep two topics near the top of the collaboration's agenda: native support for large arrays, structured data, and data compression; and development of crossplatform tools. 


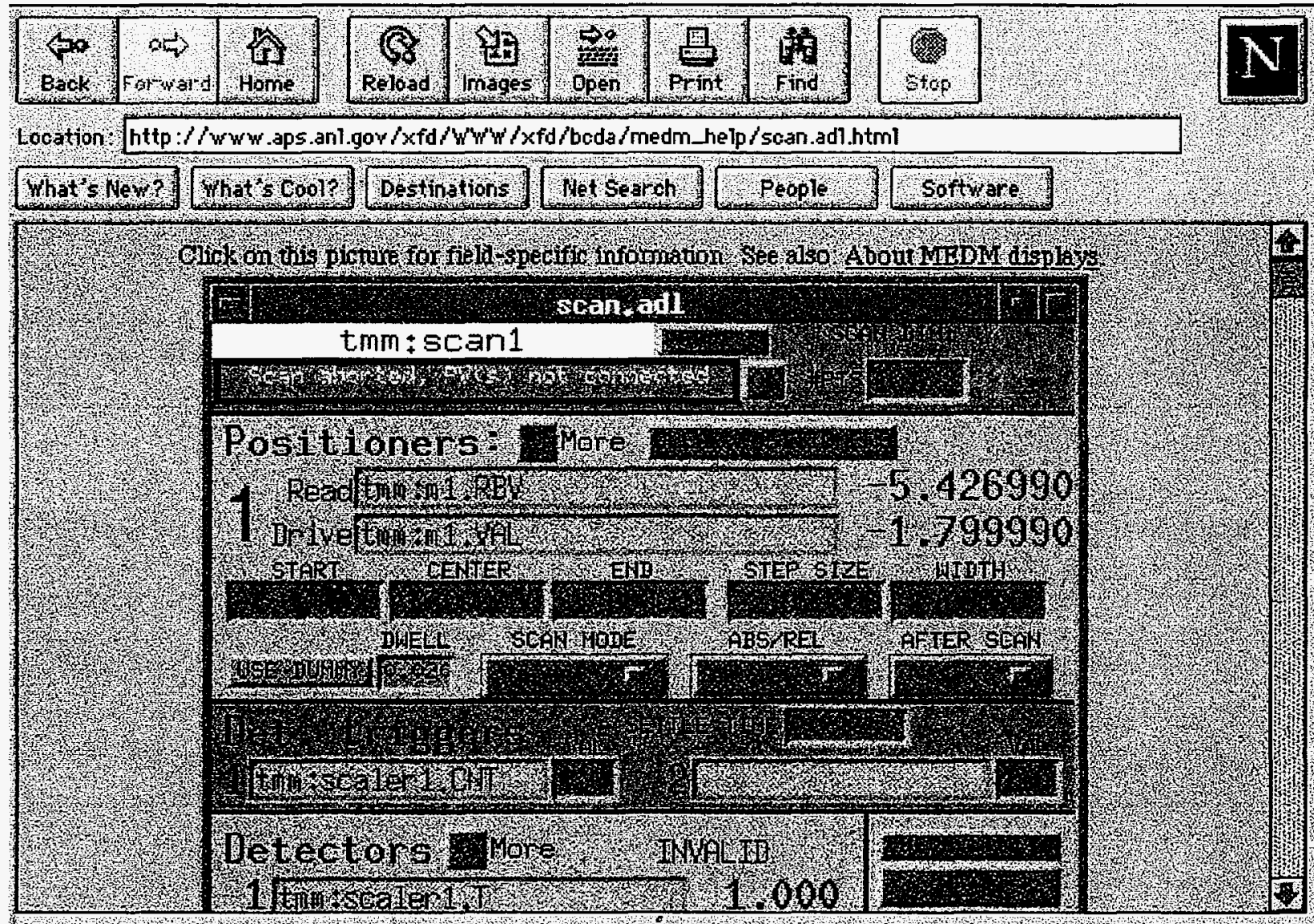

\section{Overview}

The scan software nepresented by this display runs in the VME crate: directing the movements of positioners; triggering detectors; and caching data from detectors into arrays until the scan is complete, when some client program running on a workstation or server (nomally the data catcher) will grab the data and store them to disk.

The focus of this documentation is on zppesstivy. If you'ne interested in implementation, or if you want a better mental picture of the scan-control softwane to aid you in devising unusual custom scans here's a place to start.

To setup a scan, you must name the positionen(s) and where they are to go; name the detector trigger(s); and name the variables that are to be acquired. Nomally, you need only name a positioner, since detector infomation tends to remain constant over many scans.

Here's a recipe:

\section{- Positioners}

1. Type the name of a positioner into the "Drive" field near the top of the screen (or use drag-and-drop). Any writable EFICS variable can be used as a positioner. Options: Semeral positioners

You can specify up to four positioners, although only one appears on the main scan display. All are displayed on the scan-positioners display, which you can call up with the "More" button. 


\section{User's View of Current Beamline Software}

From a user's viewpoint, EPICS-based beamline software looks like a collection of objects (motors, slits, optical tables, scalers, scans, etc.) any of which can be grabbed and tweaked, moved, etc., at any time. Associated with each object is a set of displays, with varying levels of detail, that run on the workstations. (A typical display is shown in the top section of Fig. 3.4.) These displays contain the object's fields, the numbers and character strings that describe its state (e.g., a motor's fields include its destination, current position, speed, name, engineering units, limits, backlash distance, etc.). Users think in terms of objects but actually manipulate fields; nobody cares about the distinction.

There are only three classes of objects/fields a user has to know about: positioners, detectors, and links. A positioner is any scalar quantity that can be written to, and most beamlines have tens of thousands of them, though the number of commonly manipulated positioners per beamline is more like a thousand. (Most "positioners" don't actually move anything physical. The boundaries of a region of interest in a multichannel-analyzer spectrum and the gain of a current preamplifier are typical examples of scannable positioners.) In principle, all positioners can be scanned, and all can be the targets of run-time calculations (see "virtual machines" below). Commonly scanned positioners have private scan parameters attached to them, so they can be scanned by pressing a single button. There is a distinction between positioners that move and settle in less than around a millisecond and those that require many milliseconds or more to complete a motion, but the distinction is important only in setting up scans.
Similarly, a detector is any quantity that can be read. (Vector-valued detectors currently require special handling, because EPICS lacks native support for multidimensional arrays.) Again there is a distinction important only for scans between fast detectors such as ADCs and integrating detectors that require triggering and signal completion, such as scalers and multichannel analyzers. A scan is a vectorvalued detector that requires triggering and signal completion-not fundamentally different from, say, a multichannel analyzer.

A link is the EPICS name of a positioner or a detector, coupled with an instruction that tells linked software what to do. More precisely, a link is a field into which the user can type the name of a positioner or detector, but users do not care about this distinction. Advanced users build virtual machines at run time using links. (Novices do this also, but they do not usually realize they are doing it.) In effect, links and displays containing them constitute a graphical programming language for users.

Beamline software is distributed among workstations and VME processors connected by a network. This allows an entire beamline to be controlled and monitored from any source (local or remote) without recabling. Most of the controlling software runs autonomously in the VME crates, while software tools running on the workstations merely provide a graphical user interface and collect cached data. VME crates periodically store their states to the file server and restore them during reboots. Usually, they can crash or be rebooted during an experiment with minimal or no consequences for the user (although scans cannot be continued through a reboot). Workstations can nearly always be rebooted without affecting the state of an experiment. 
Control of VME-resident software is shared, so that several independent users (and software tools, such as the diffractometercontrol program, SPEC) can operate overlapping sets of beamline equipment. This allows graphical and command-line user interfaces to be active simultaneously, both with full and independent access to beamline hardware. It also allows a remote user to collaborate effectively in an experiment. Currently, collisions between the various command streams that share control of a beamline are handled naively. Although EPICS has a good access-control system, we have not had time to apply it.

The VME-resident software can be modified, within limits, at run time. This allows users to build virtual machines that run autonomously in the crate. (Examples: the user can slave a voltage output to an arbitrary function of the beam current and beam position; the user can arrange to disable one motor whenever another motor is moving, is within a specified range of positions, has hit a software limit, etc.; the user can implement a theta/2-theta coordination and scan it as though it were a simple motor.) The latest version of EPICS also allows developers to change any motor assignment at run time (to work around failed hardware, for example). We have not yet addressed the user-interface details that will allow users to do this.

Actual accomplishments in software development and support are probably beside the point of this report, because software is not the end product here. However, by design, many of our activities are not obvious in the user's view of beamline software presented thus far (our real accomplishment), but reviewers should nevertheless know what we've been doing. Here is a list of last year's highlights:
- Responded to roughly 5000 requests for technical support, ranging from simple requests for vendor information to installation of EPICS and beamline software on a CAT's file server.

- Set up and outfitted network-support and beamline-control labs.

- Performed system and network administration for six beamlines and related labs.

- Installed and configured standard beamline software in 15 XFD labs and ten SRI-CAT experiment stations.

- Performed extensive trouble shooting of the Oregon Micro Systems VME58 step-motor controllers, and loaded new firmware into 20 boards.

- Designed and tested 190 motorcontroller signal-transition boards (135 of these boards are now in service at APS).

- Made many improvements to the data catcher, the program that displays and stores scan data.

- Developed a data browser for hierarchical data format (HDF) files, and investigated HDF performance. (HDF is the standard selected by a collaboration including developers from APS and several neutronscattering facilities to underly a common data-file format.)

- Developed Web pages for hardware technical support, distribution of 
EPICS and beamline software, online documentation of beamline software, and an EPICS learning system for users and developers.

- Hosted beamline-controls workshop and collaboration meetings, EPICS users and developers classes, and an IDL class.

- Developed software support for the following devices:

- HP laser interferometer

- Moller-Wedel autocollimator

- Laser Doppler angle encoder

- Lakeshore temperature controller

- Various single and double-crystal monochromators

- Mirrors, slits, and filters

- Various incremental-encoder interfaces

- Keithley scanning multimeters

- Multichannel-analyzer regions of interest

\subsubsection{Leveraging of APS Funds}

The IDs and FEs for the 20 APS user sectors that are currently under development represent a total investment by the APS of about $\$ 50$ million. The CATs have leveraged this investment significantly by raising an additional $\$ 155$ million to construct and instrument the beamlines outside the shield wall in these sectors. A breakdown of the CATs' funding sources is given in Fig. 3.5. The XFD staff has assisted many of the CATs in their fundraising efforts by working with them to develop designs and cost estimates and by providing information to their program managers during site visits.

\subsubsection{User Procurements of Standard Components}

The APS beamline standard components designs are available to the APS users through the Design Exchange, as described earlier. A library with over 180 components that have been designed, tested and installed is available to the users to procure either as they are or modified to meet the requirements of their beamline designs. All contracts to procure components for the APS beamlines were signed with an option clause that can be exercised to purchase up to 10 times the quantity procured within a year from the date the contract was signed. The following types of components are available in the DX library:

- Enclosures:

- First Optics Enclosures

- White Beam Experiment Stations

- Monochromatic Experiment Stations 


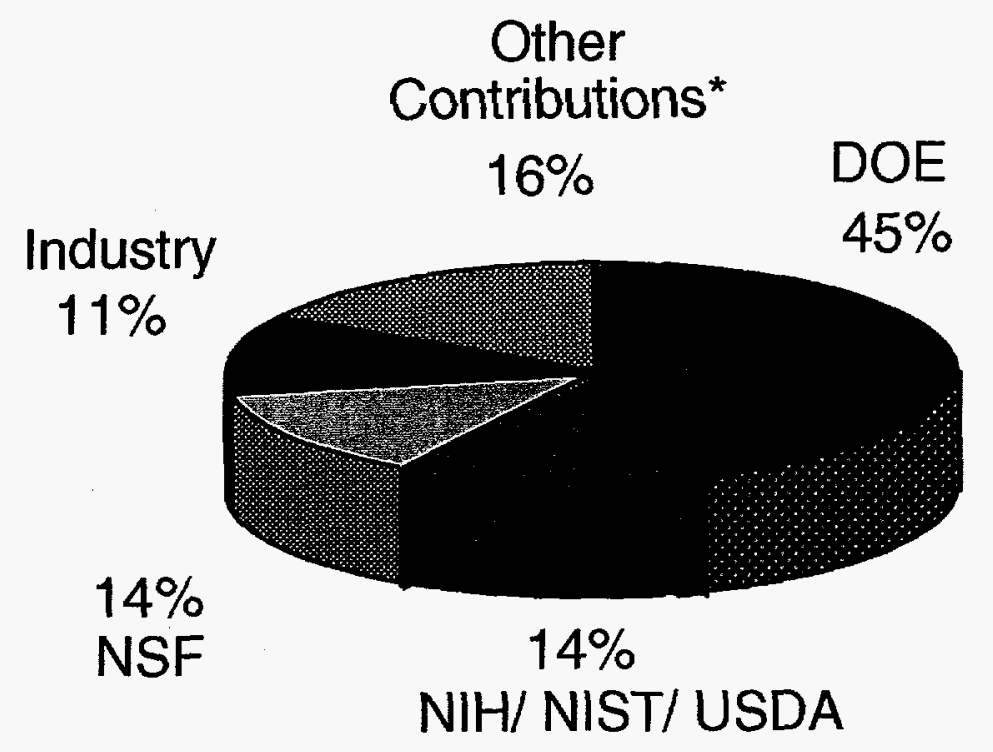

\section{TOTAL FUNDING $=\$ 155 \mathrm{M}$}

* Funds from States, Foundations, Foreign Countries

Fig. 3.5 Funding sources for APS Collaborative Access Teams

- Beamline Transports:

- Shielded Pipes

- Shielded Cabinets

- Shielded Supports

- Collimators

- Tables (Support Structures)

- Windows

- Filters

- Slits
- Integral Shutters

- Beam Position Monitors

- Photon Masks

A process was initiated by XFD to support the user procurements and ensure that the delivered components meet the user's requirements. Once the user beamline design is reviewed by the XFD Beamline Review Committee, the procurement process starts.

Users who wish to exercise an option on the APS designs can view a comprehensive list of all the various types of components along with the option price and the time for its delivery from the vendor. The request is then 
submitted to the User Technical Interface Office, where it is forwarded to the XFD Project Engineer. A meeting is then held to review the user's requirements. Participants in the meeting include the cognizant engineer who designed the component, the installation coordinator, the purchasing agent, the User Technical Interface Group Leader, and the XFD Project Engineer. The responsibilities carried out by the participants are described in Fig. 3.6. Once the procurement process starts, biweekly status reports are issued to the user.

The users have gained the following benefits through this process.

- The components have been designed, and prototypes have been built and tested.

- Trained vendors have been established.

- Inspection procedures have been well established, guaranteeing quality product.

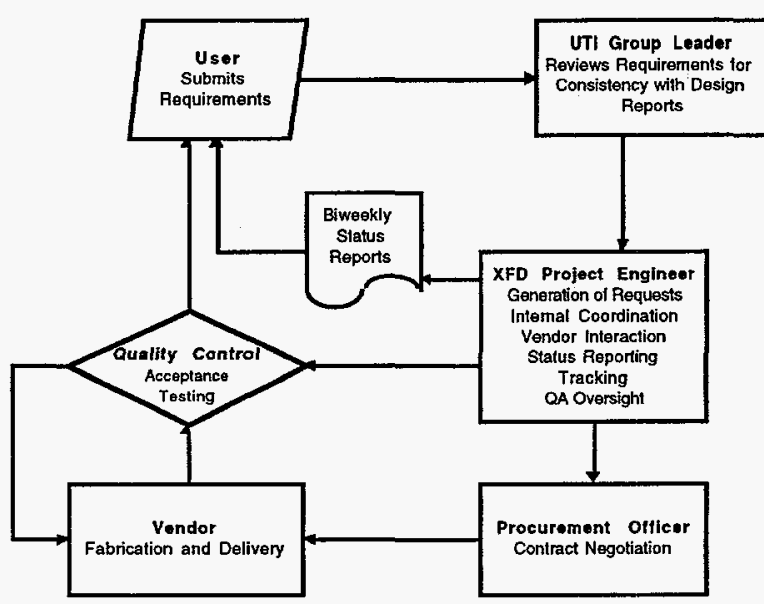

Fig. 3.6 User Procurement Flow Process
- The process assures the safe operation of the component and the beamline.

Through the use of the above-mentioned process, the cost savings to the users was over 12 million dollars. To date the users have ordered over 300 standard components through the APS for a total of 13 million dollars.

\subsubsection{The Inter-CAT Technical Working Group}

The Inter-CAT Technical Working Group (TWG) was established by the CATs in September 1994 to facilitate CAT-APS and CAT-CAT interactions and information exchange on technical issues, identify common CAT needs, and promote sharing of intellectual resources among the CATs. Each CAT has designated one or more TWG representatives, who meet on a monthly basis; generally, 20-30 CAT members attend each meeting, along with the XFD User Technical Interface Group Leader. Jim Viccaro (CARSCAT) was the first TWG Chair; in January 1997, he was succeeded by the current co-Chairs, Dean Chapman (Center for Synchrotron Radiation Research and Instrumentation, Illinois Institute of Technology) and Dean Haeffner (SRI-CAT).

A regular feature of TWG meetings is the "APS facility update." Topics presented by XFD staff as part of these updates have included the following:

- Performance of high heat load optics

- Performance of data acquisition systems 
- Liquid nitrogen distribution options

- Specifying and installing the Personnel Safety System on experiment stations

- X-ray optics metrology and fabrication capabilities available to users

- APS vacuum policy

- APS-supplied equipment and layouts for User Shops

- Using the APS Design Exchange

\subsubsection{CAT Chats}

In September 1995, XFD initiated a series of weekly "CAT Chats," informal Friday afternoon meetings that give CAT members an opportunity to present questions and issues directly to XFD management. The issues discussed at CAT Chats have covered a wide range of technical, administrative, safety, and user service topics. If a question cannot be answered on the spot, XFD provides an answer at the following meeting. In addition, at the beginning of each meeting the XFD Associate Division Director for Operations gives an update on the operations schedule, shielding validation activities, etc., for the coming week. The updates, questions, and answers are compiled into weekly minutes, which are distributed at the subsequent meeting and posted on the Web. To date, 65 CAT Chats have been held.

\subsection{References}

Assoufid, L., and D.M. Mills, SRI CAT Newsletter, Vol. 3, No. 2 (1997) 4-7

Bresloff, C., and D.M. Mills, Rev. Sci. Instrum. 67 (9) (1996) CD ROM

Khounsary, A. M. and W. Yun, Rev. Sci Instrum. 67 (9) (1996) CD ROM

Kuzay, T. M., Argonne National Laboratory Report ANL/APS/LS-187 (1992)

Lai, B., D.C. Mancini, W. Yun, and E. Gluskin, SPIE Proc. 2880 (1996) 171-176

McNulty, I., A. Khounsary, J. Barraza, C. Benson, Y.P. Feng, and D. Shu, Rev. Sci. Instrum. 67 (9) (1996) CD ROM

Randall, K. J., E. Gluskin, and Z. Xu, Rev. Sci. Instrum. 66 (8) (1995) 4081-4086

Shu, D., J. Barraza, C. Brite, T. Sanchez, and V. Tcheskidov, Rev. Sci. Instrum. 66 (1995) 1795-1797

Yun, W., A.M. Khounsary, B. Lai, K.J. Randall, I. McNulty, E. Gluskin, and D. Shu, Rev. Sci Instrum. 67 (9) (1996) CD ROM 
3 USER ADMINISTRATION AND TECHNICAL SUPPORT 

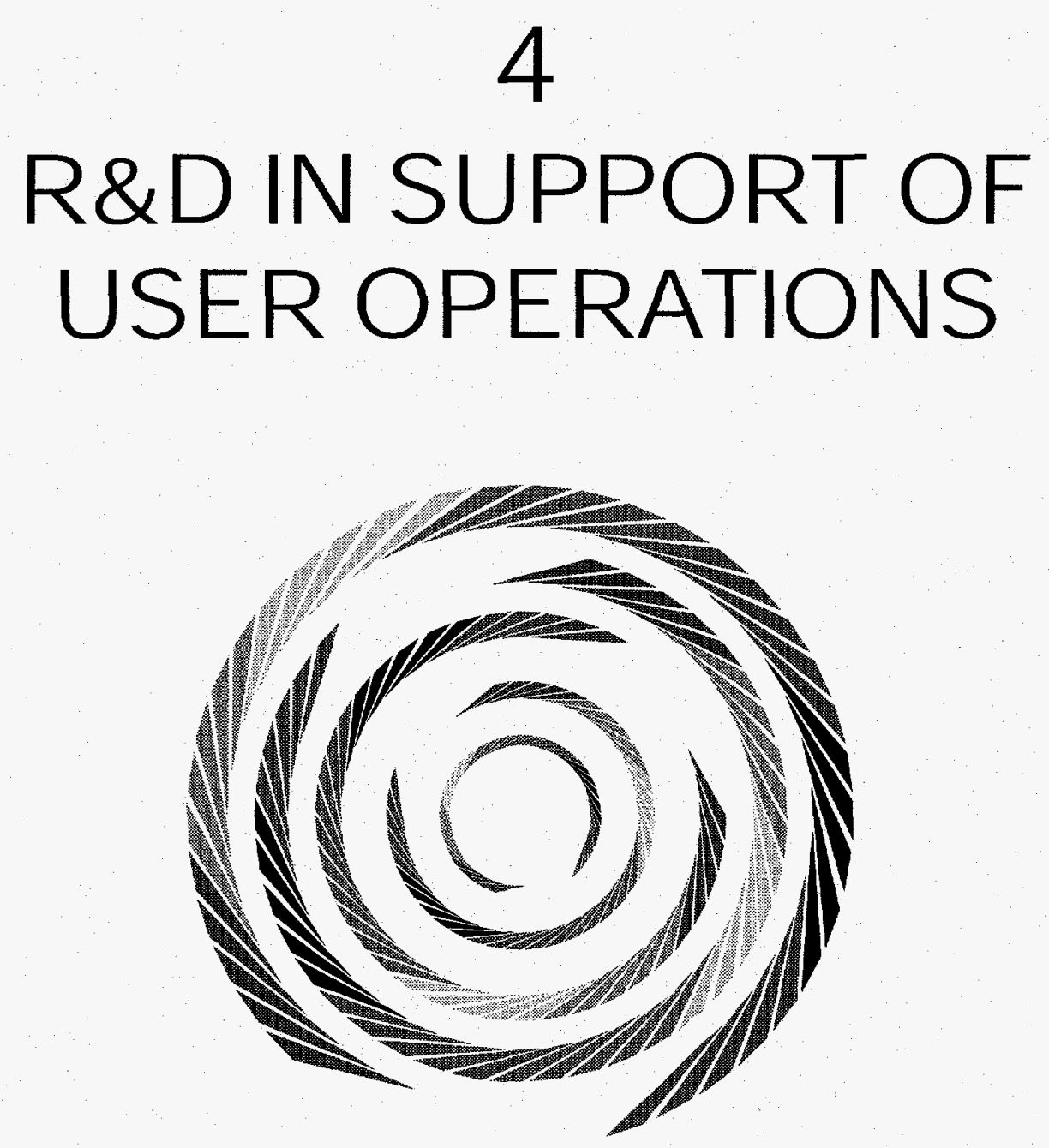


\subsection{Radiation Sources}

The main objective of the $\mathrm{ID}$ effort is to perform $R \& D$ to improve ID performance and meet new user needs for radiation with special characteristics and to handle major breakdowns of operating IDs.

A strategy for ID development was conceived in the early years of the APS project: to develop and build a universal ID that could provide radiation over a large energy range, 5 to $25 \mathrm{keV}$, without compromising the record high brilliance expected from a third-generation source. In order to achieve this goal, state-of-the-art techniques and equipment needed to be developed.

High brilliance could only be delivered if the magnetics of the $\mathrm{ID}$ were properly designed and were accurately tuned after fabrication. The precise magnetic tuning, in turn, required an advanced measurement system.

The wide energy range to be covered by the ID dictated a high peak magnetic field that could be varied in a precise and controllable manner. This led to the need for sophisticated controls and innovative vacuum systems with small vertical apertures.

Finally, experimental verification of the design and fabrication of the ID required a set of diagnostic equipment capable of measuring the absolute spectral flux under the conditions of record high x-ray power density. The results of these measurements would need to be compared to theoretical calculations.

The progress in realizing these goals is recounted in the remainder of this section of the report.

\subsubsection{Magnetic Measurements and Tuning of IDs}

It is imperative that APS users be provided with a stable $x$-ray beam. Nothing must perturb the x-ray beam by more than a few percent of its size and angular divergence. However, the $\mathrm{x}$-ray beam can be perturbed if there is a change in the steering of the positron beam through an undulator. A small change in the positron beam steering could cause a large change in the position of the photon beam at the user's experiment due to the long distance between the source and the experiment. If the magnetic field of an ID is not well tuned, changes in the ID gap could cause changes in the positron beam orbit, either through that ID or through another ID elsewhere on the ring. In order to allow the users the freedom to change their ID gaps, the IDs must be very well tuned magnetically so that they do not perturb the stored beam orbit and thereby affect the stability of any user's x-ray beam. The specifications for the IDs called for a very high quality magnetic field, and the insertiondevice vendor more than met these requirements. However, the requirements were determined based on the assumption that there would be an active local feedback system to partly compensate for the remaining field errors. Further refining of the tuning of the IDs has been carried out in order to reduce or even eliminate the need for the active local feedback.

Another goal of tuning the magnetic field of an undulator is to improve the quality of the spectrum of emitted photons. Again, the undulator vendor more than met the demanding requirements, but the phase error has been further reduced so as to improve the spectral performance over a wider range of energies. The phase error does not cause a large decrease in the first harmonic intensity, but the amount by which it decreases the 
intensity in a given harmonic increases with the harmonic number. In fact, it goes as $\mathrm{e}^{-\mathrm{np}} \mathrm{p}^{2}$, where $\mathrm{n}$ is the number of the odd harmonic and $p$ is the phase error in radians (Walker, 1993). Therefore, the effect of a $5^{\circ}$ phase error on the 5 th and 7 th harmonics would be to reduce the intensity to $83 \%$ and $69 \%$ of the ideal intensity, respectively.

An essential component of the ability to tune IDs is the ability to make accurate measurements of their magnetic field. The ID Magnetic Measurement Facility (Burkel et al., 1993; Frachon et al., 1995) now is equipped with two magnetic measurement benches, one with $3 \mathrm{~m}$ of travel and the other with $6 \mathrm{~m}$ of travel. There is also a laboratory electromagnet and a prototype APS dipole magnet for calibrating coils and Hall probes. A variety of probes can be mounted on either bench so they can map out the magnetic field in the gap of an ID. These probes include a number of coils of various lengths and orientations and Hall probes. A recent addition to the probes is an axial Hall probe that is used to measure the transverse horizontal component of the magnetic field. Long, stretched-wire-type rotating coils are also mounted to each bench so that integrals of the field through an ID can be measured more directly than with point-bypoint measurements. The reproducibility that is routinely attained for the first field integral measurements is better than $0.5 \mathrm{G}-\mathrm{cm}$. Using a Hall probe, the first field integral reproducibility is better than $5 \mathrm{G}-\mathrm{cm}$. That the reproducibility of the measurements also applies in the long term has been shown by Hall-probe measurements of an ID that repeated with an rms difference of $2 \mathrm{G}-\mathrm{cm}$ after a period of one year.

In order to refine the magnetic field, tuning techniques have been developed. After the alignment of the magnetic structures has been checked and adjusted as necessary, the primary means of tuning the magnetic field is shimming. Different sizes, shapes, and placements of small shims are used to adjust different aspects of the magnetic field. For most of the tuning techniques, the shims are placed on top of the magnets rather than on the poles because the magnetic structure was designed with the magnets recessed by a small amount compared to the poles. This allows shims to be placed on the magnets without affecting the achievable minimum gap. Shims can be placed along the $\mathrm{ID}$ as needed to straighten the trajectory and to decrease the phase error, thus helping to ensure a high quality undulator spectrum (Vasserman, 1996).

One way in which an ID can affect the stored beam is if the first or second integral of the horizontal or vertical component of the field through the ID is substantial. Therefore, an important goal in the tuning of an ID is to minimize these integrals, and to minimize the change in the integrals as the gap of the ID is changed. None of the standard planar IDs have any active correction of the field integrals, so the users rely on this tuning for a stable beam in the ring despite someone else's ID gap being changed. A non-zero first integral means that the trajectory of a particle after it has passed through the ID is at an angle with respect to its incident trajectory. A nonzero second integral means that the trajectory is offset after passing through the ID. The second integral is also a measure of the average trajectory angle inside the ID, so that if it varies with gap then the angle of the emitted photon beam will also vary, and the user will see the beam move as the gap is changed.

Once the trajectory through the ID is reasonably straight, first and second integral tuning is accomplished mainly by changing the configuration at the ends of the ID. A number of aspects of the end configuration are used in the tuning, including adjusting the 
strength of the end magnets, changing the height or shape of the last two poles (in ways that do not affect the vacuum chamber clearance at minimum gap!), or adding shim material to the gap faces of the magnets or between two magnets on the side of a pole. Each of these changes has its own effect on the magnetic field integrals, and its own gap dependence to the effect. With some of the changes, the size of the effect varies even between nominally identical IDs, so it may be difficult to know a priori what will work best.

The IDs are also tuned to minimize their integrated multipole moments. If the integral of the field through the $\mathrm{D}$ possesses a substantial quadrupole, sextupole, or octupole moment, that could affect the focusing and/or coupling of the particle beam.

\subsubsection{Field Quality Achieved}

Tuning techniques have been applied to finetune the magnetic fields of all the IDs. As more experience has been gained in tuning, the field quality achieved has improved. Over the past several shutdowns, installed IDs have been removed from the storage ring in order to install an improved design for the maximum gap hard stop. While the devices have been out of the ring, further refinement of the field quality has been carried out. This effort will continue as techniques improve. Fig. 4.1 shows the total variation of the vertical and horizontal first and second integrals of the field over the entire gap range, for the various IDs as they are now. Fig. 4.2 shows the phase errors for all of the undulators at a magnetic gap of $11.5 \mathrm{~mm}$. The integrated multipoles through the IDs are also of importance due to the effect they have on the focusing and coupling in the stored particle beam. Fig. 4.3 shows the size of the variation in the normal and skew integrated quadrupole, sextupole, and octupole moments for the IDs.

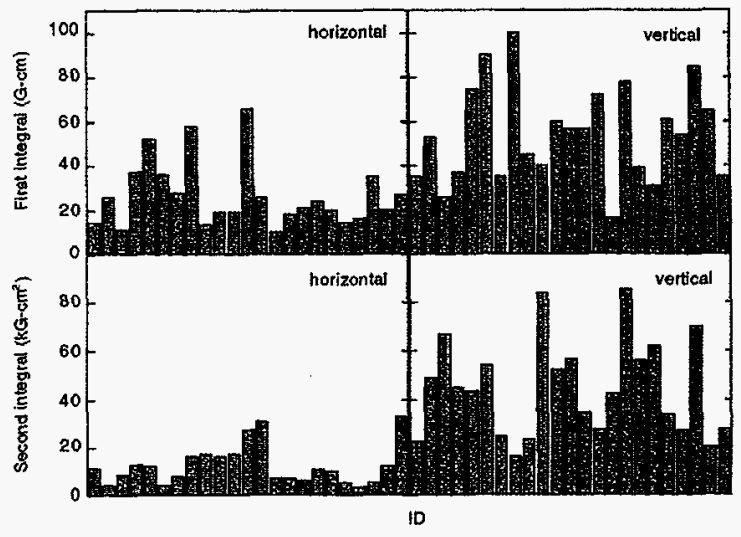

Fig. 4.1 The first and second integrals of the vertical and horizontal components of the magnetic field through the IDs are measured and tuned over the full gap range. The maximum and minimum values reached by each integral are determined. This figure shows the difference between those maximum and minimum values, for each ID.

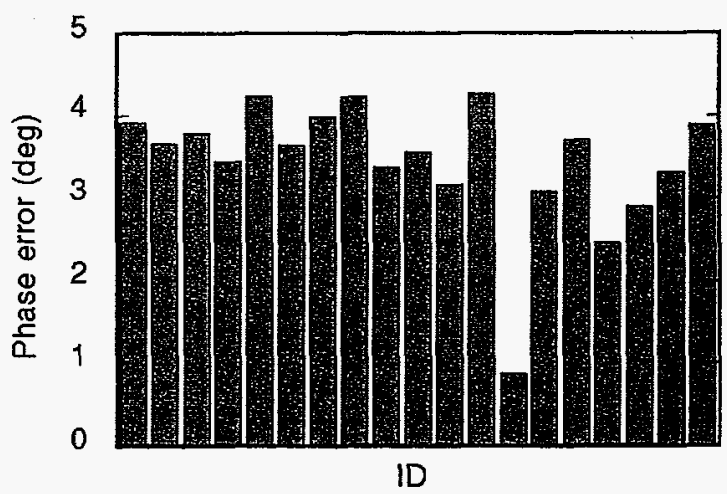

Fig. 4.2 The phase error at a gap of $11.5 \mathrm{~mm}$, as measured for all of the APS undulators.

\subsubsection{ID Control Development}

Insertion-device control system development is focused on extending control access to users, improving ease of use, expanding the control system to accommodate new types 


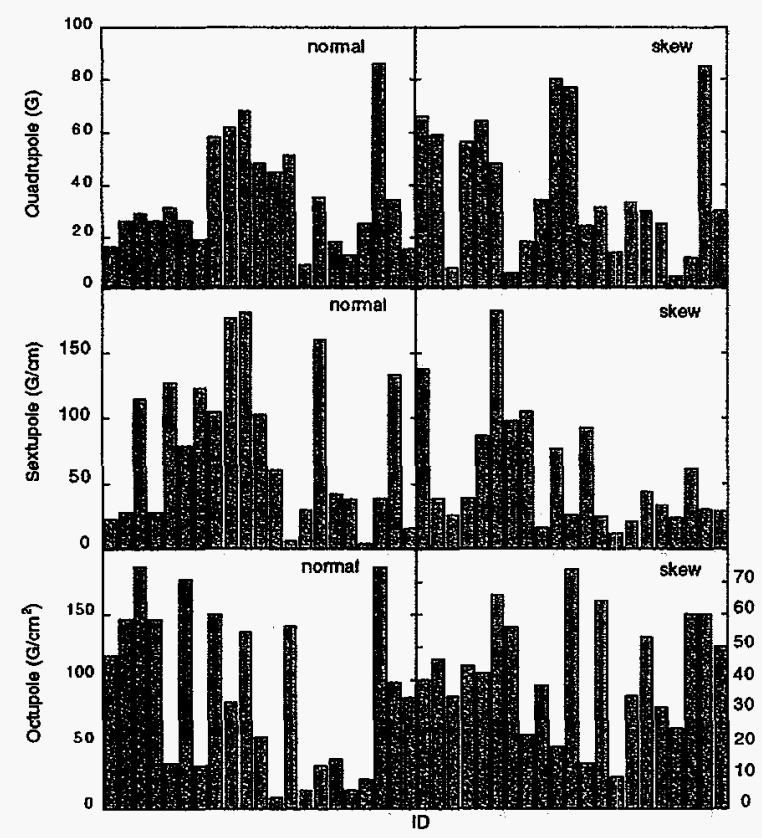

Fig. 4.3 The integrated multipole moments of the magnetic field through the IDs are measured and tuned over the full gap range. Maximum and minimum values for each moment are determined. This figure shows the difference between those maximum and minimum values, for each $I D$.

of IDs and improving system reliability. Eighteen undulators have been installed in the storage ring: 16 undulators $\mathrm{A}, 1$ wiggler $\mathrm{A}$, and 1 elliptical multipole wiggler.

The control of the IDs installed into the storage ring is integrated into the APS EPICS computer network with a dedicated IOC for each straight section. Controlled parameters include upstream and downstream drive motors that allow parallel or tapered positioning of the magnetic structure, linear absolute encoders for position monitoring, absolute rotary encoders for redundancy, and protective limit switches and interlock circuitry. Operators and users can monitor status and initiate actions from the control room or from computers located in offices or on beamlines. Security is maintained by the use of a process variable server that allocates control according to predefined access tables.

The control system has been designed to enable users to control the ID on their own beamline simply and easily without involving facility operators. To this end, the control system has been developed to be friendly and easy to use but to be rugged and completely self protecting. Users can select a parallel gap position, a tapered gap position, or a desired first harmonic energy. The IDs have been designed and carefully tuned to eliminate any coupling between beamlines. Tests have shown that ID gap changes can be made in any or all sectors simultaneously without impacting the other users. All possible failure modes are continuously monitored, and users are prevented from inadvertently moving the ID in a manner that could damage the ID or the storage-ring vacuum system. The IDs are protected against motor stalls, encoder failure, excessive taper, and exceeding minimum and maximum limits. A software limit for commissioning can be set by the Floor Coordinator to administratively protect against excessive $\mathrm{x}$-ray power during the commissioning of a beamline. If a problem should arise, the system will latch in its current state and a message will alert a user to the need to notify a Floor Coordinator to obtain expert assistance. An automatic log records the status changes and any errors that occur.

The interface to the ID control is implemented with the channel access features of EPICS. Control screens have been provided for users operating UNIX systems with EPICS (Fig. 4.4), or alternatively, users are able to design their own screens using variable names and descriptions that are readily available through the XFD Operations home page on the World Wide Web. CATs are asked to 


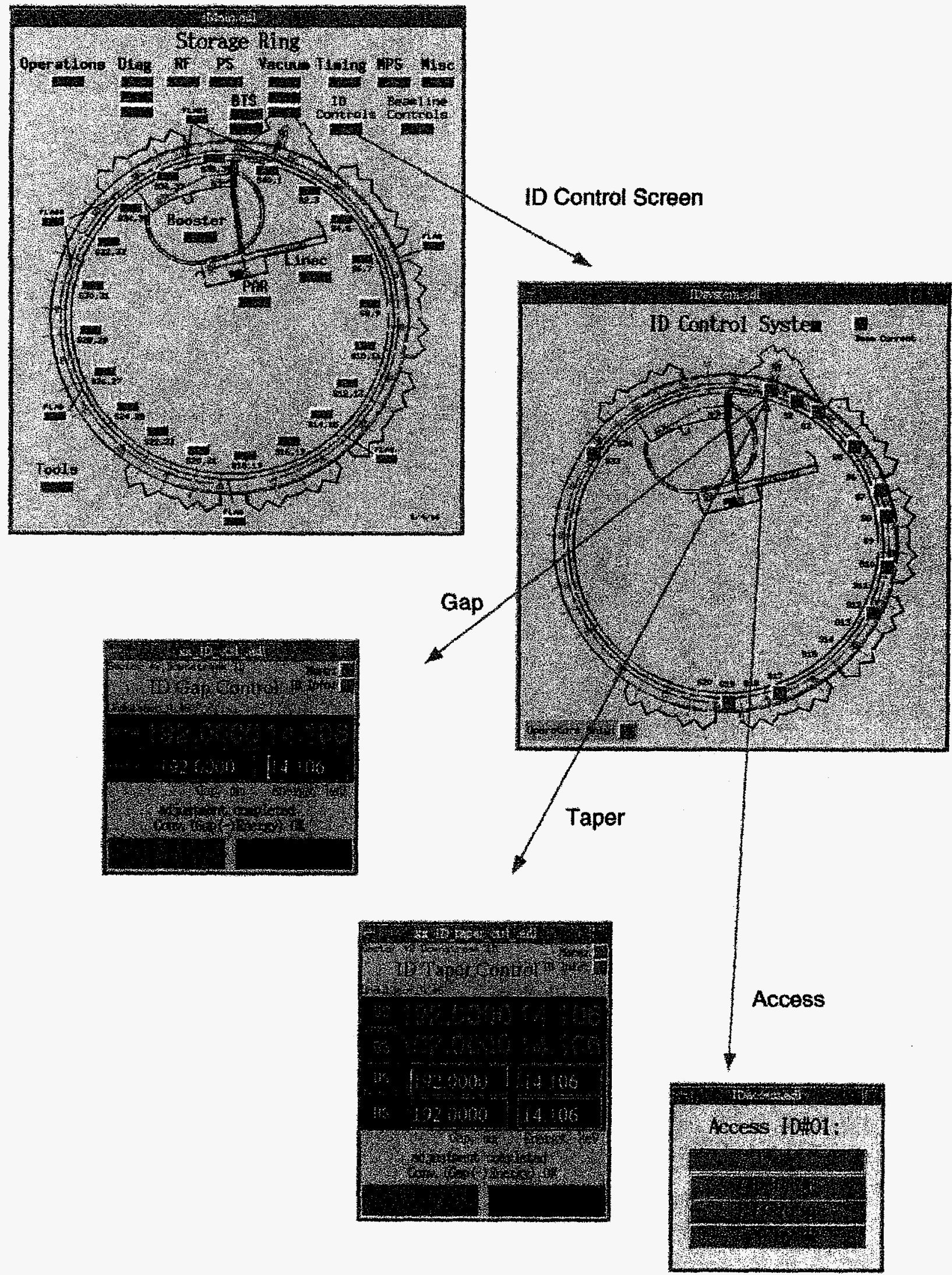

Fig. 4.4 EPICS screens available to users for control of undulator A and wiggler A. 
provide a list of computer names and user names that are to be given access for control of the ID for their beamline. Only these authorized persons, Floor Coordinators, and system maintenance personnel are able to change gap positions.

The elliptical multipole wiggler (EMW) is a unique ID that has been installed in Sector 11 of the storage ring for polarized $\mathrm{x}$-ray studies. In addition to the hybrid permanent magnet structure, it contains a horizontal field electromagnet that can alternate polarity at up to $10 \mathrm{~Hz}$. The EMW control system consists of the standard controls for the vertical gap along with controls for the 1000 amp electromagnet supply and correcting coil supplies. The correcting coils are energized through an arbitrary function generator that allows the field to be varied so as to reduce the field integral of the electromagnet at positive and negative polarity and during the transitions. EPICS control screens have been generated to allow the EMW operator to vary all of the parameters during commissioning, but further work is required to reduce the controls to the relevant set for casual users and to simplify execution.

\subsubsection{ID Vacuum-Chamber Development}

A number of considerations in the design of undulators drive the design of the vacuum chamber to small gaps. On the other hand, particle beam transport considerations require the largest possible aperture. The competing requirements demand a vacuum chamber design with minimum chamber wall thickness, close tolerances for straightness and flatness to enable precision alignment, as well as mechanical and thermal stability. In addition, a low outgassing rate, achievable manufacturability, low maintenance, and high reliability are also desirable.

During the last five years, a new approach to the design and fabrication of extruded aluminum vacuum chambers for IDs was developed at the APS (Trakhtenberg et al., 1996). Versions of the vacuum chamber, with vertical apertures of $12 \mathrm{~mm}$ and $8 \mathrm{~mm}$, and lengths of 2.5 meters and 5 meters were manufactured and tested. Twenty chambers were installed in the storage ring and successfully integrated into the APS vacuum system. All have operated with beam, and 16 have been coupled with IDs.

All of these vacuum chambers have a wall thickness of $1.0 \mathrm{~mm}$ at the beam orbit position. Figure 4.5 shows a typical cross section. The chambers are fabricated by extruding 6063 aluminum alloy to form a tube with the desired internal shape (a) and machining the exterior to finish dimensions (b) and (c). The design utilizes a rigid strongback that limits deflection of the chamber under vacuum despite the thin wall.

In order to achieve and control the required vacuum, the ID vacuum chamber is equipped with a set of vacuum pumps and vacuum gauges. The total pumping capacity is achieved by combining two $\sim 5$-meter-long nonevaporable getter (NEG) strips with an average pumping speed of $\sim 6 \mathrm{~W}(\mathrm{sec} \mathrm{cm})$, a $30 \mathrm{l} / \mathrm{sec}$ ion pump, and a $220 \mathrm{l} / \mathrm{sec}$ lumped NEG pump in each end box.

In addition to the pumps, the end boxes also accommodate transition sections, an $\mathrm{x}$-ray absorber, and vacuum analyzers. The transition section in the upstream box is a watercooled copper block that provides a smooth 


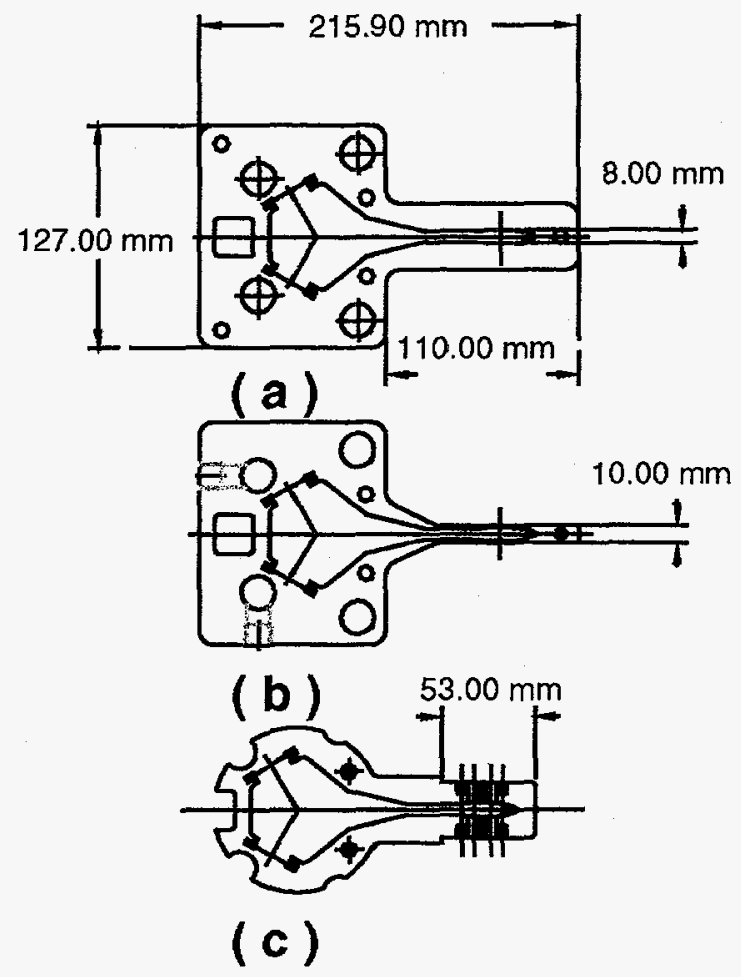

Fig. 4.5 Cross section of the $8 \mathrm{~mm} \mathrm{ID}$ chamber. (a) extrusion, (b) after machining, (c) end geometry showing the locations of beam position monitors.

transition between the aperture of the ID and storage-ring vacuum chambers. The transition block in the downstream box is not water cooled. Both transition sections must be installed to avoid impedance mismatches that would affect the positron beam. The $\mathrm{x}$-ray absorber is located in the downstream end box. It prevents the bending-magnet synchrotron radiation from penetrating the vacuum valve. Thermal calculations show, and experiments with installed chambers verify, that under relatively moderate cooling conditions, the temperature rise on the surface of the transition block or on the $\mathrm{x}$-ray absorber does not exceed $110^{\circ} \mathrm{C}$ with a $300 \mathrm{~mA}$ positron current in the storage ring and under maximum x-ray beam missteering. Figure 4.6 shows the layout of the $\mathrm{ID}$ vacuum chamber.

During the last year, 14 aluminum ID vacuum chambers were installed in the storage ring. Each chamber was certified at a pressure below $2 \times 10^{-10}$ Torr prior to installation, and vacuum performance of the chambers was monitored with and without beam present. Initial installed pressure without beam is typically $1-5 \times 10^{-10}$ Torr (Fig. 4.7). Photodesorption from synchrotron radiation raises the pressure during beam operations to about $2 \times 10^{-9}$. After several weeks of beam conditioning, pressures during beam operation decline to $<1 \times 10^{-9}$ (Fig. 4.8 ).

Alignment of the vacuum chamber on its support is routinely accomplished using optical techniques to a precision of $\pm 75 \mu \mathrm{m}$ over the entire surface. This allows minimum insertion-device pole gaps to be obtained. All of the installed undulators can achieve a minimum gap of $10.5 \mathrm{~mm}$ while maintaining clearance from the chamber. At the present time, the minimum gap of all chambers is administratively set to $11.0 \mathrm{~mm}$. As more experience is gained with the $\mathrm{ID}$ controls and the mechanical structure of the gap separation mechanism, the minimum allowed gap will be decreased to the mature phase value of $10.5 \mathrm{~mm}$.

Experience elsewhere with stainless steel chambers and ST707 NEG material suggested the possibility of levitating dust particles of ferromagnetic material into the beam aperture of the ID vacuum chamber during ID operations. The signature would be a sudden drop in the lifetime of stored beam due to collisions with the levitated particle. No evidence of this effect has been observed at the APS. 


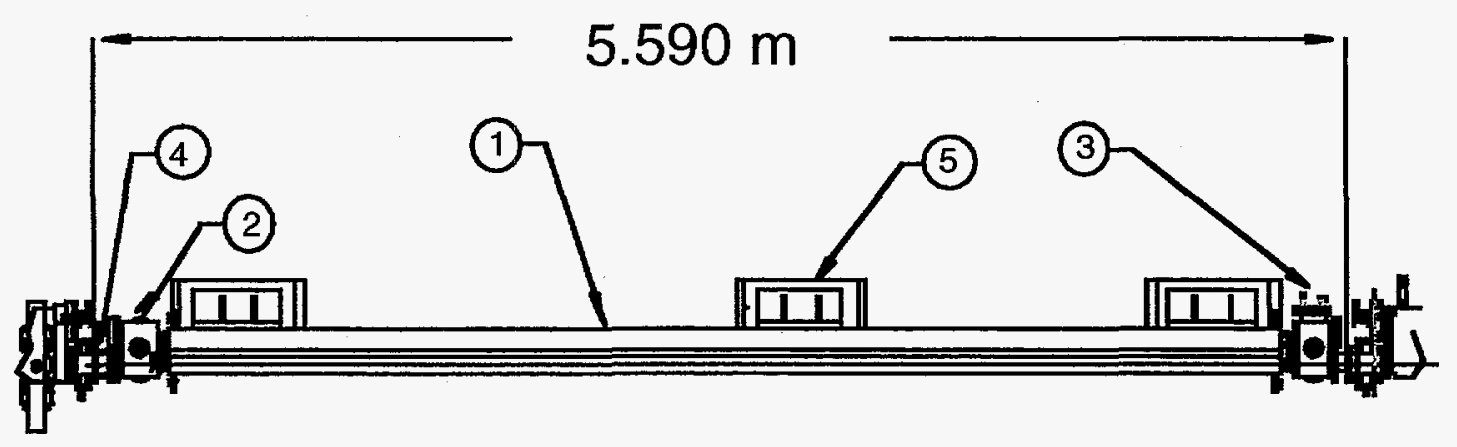

Fig. 4.6 Layout of the $5 \mathrm{~m} I D$ vacuum chamber. (1) ID chamber, (2) (3) stainless steel end box, (4) bellows, (5) support structure.

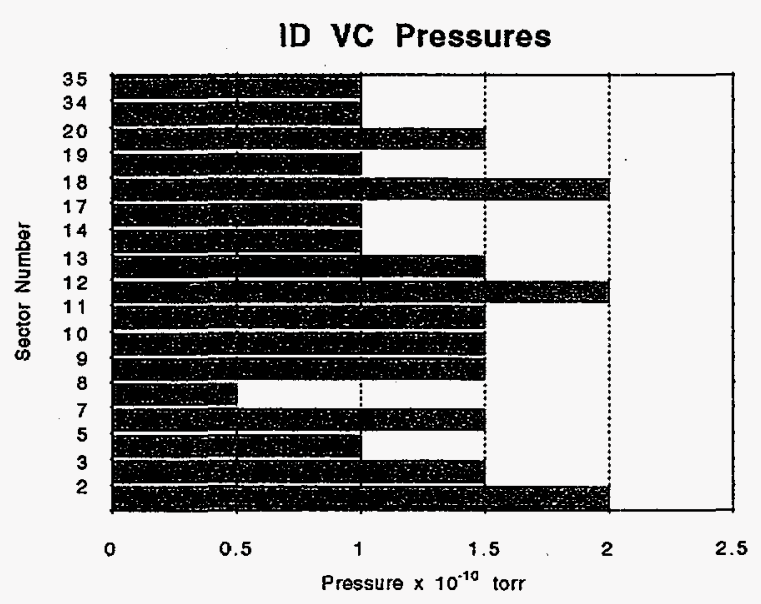

Fig. 4.7 Typical pressures for ID vacuum chambers installed in the storage ring.

\subsubsection{5-mm-Aperture ID Vacuum Chamber}

The standard 8-mm-aperture chamber with $1 \mathrm{~mm}$ wall thickness allows a minimum gap of $10.5 \mathrm{~mm}$ after allowing $0.250 \mathrm{~mm}$ on either side of the chamber for variations in straightness, flatness, and ID pole height variations. Because the gap separation mechanism of undulator $\mathrm{A}$ is designed to be able to withstand the magnetic forces at a minimum gap of $8.5 \mathrm{~mm}$, it is desirable to have a vacuum chamber that takes full advantage of the

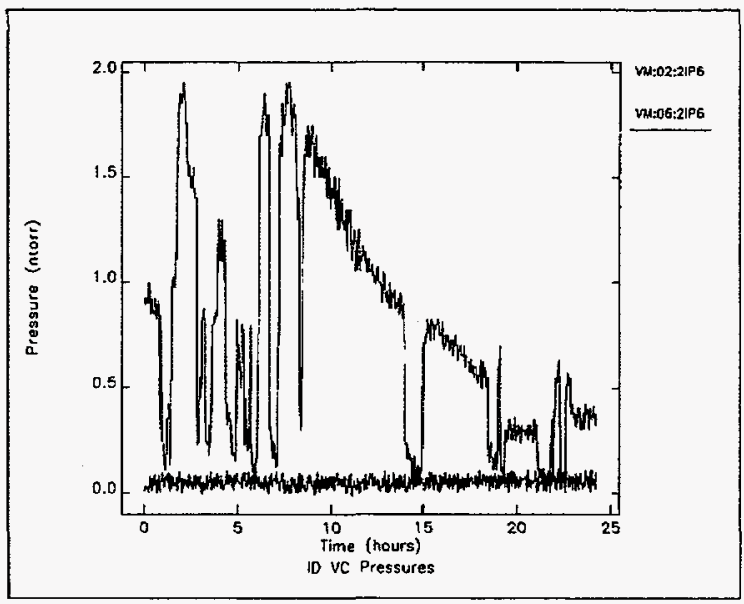

Fig. 4.8 Effect of beam-induced photodesorption on an ID vacuum chamber. The upper trace is for a newly installed chamber in Sector 6. The lower trace is for a previously installed and well-conditioned chamber in Sector 2. The maximum stored current was $80 \mathrm{~mA}$.

capabilities of the ID. A 5-mm-aperture extrusion was designed and fabricated and successfully machined to the same exacting tolerances as the standard 8-mm-aperture chamber. The chamber end geometry was tapered to $12 \mathrm{~mm}$ aperture to enable use of the standard end box. The chamber was welded, assembled, certified for vacuum, and 
completely prepared for installation into the storage ring. Current plans call for a test installation in the storage ring in an unoccupied sector sometime during late 1997 or early 1998. A decrease in the size of the NEG pumping channel in this chamber relative to the standard $8 \mathrm{~mm}$ chamber results in somewhat worse vacuum conductance. During the test, the positron beam behavior will be monitored closely to detect any adverse effect on the beam lifetime or emittance. After the tests, the chamber will be removed from the storage ring for reinstallation in a sector where the full capabilities of the undulator $\mathrm{A}$ are needed.

\subsubsection{EMW Vacuum Chamber Development}

As described previously, the EMW utilizes both a vertically oriented permanent magnetic field and a horizontally oriented electromagnetic field. The design incorporates a 3100-mm-long stainless-steel vacuum chamber of rectangular cross section, with outer dimensions of $69 \mathrm{~mm}$ wide by $22 \mathrm{~mm}$ high outer and a wall thickness of $1.2 \mathrm{~mm}$ (Den Hartog et al., 1996). Figure 4.9 shows the $5 \mathrm{~m}$ straight-section vacuum chamber assembly for the EMW. The magnet structures completely surround the chamber on all four sides, positioned only $1 \mathrm{~mm}$ from the chamber when in use. This precludes use of an "antechamber," as used in the standard insertion-device vacuum chamber. The standard chamber uses electrically activated NEG strips running the length of the chamber on the antechamber side for UHV pumping.

Given the constraints of the magnet geometry and the chamber length, two approaches were pursued for effective UHV performance. Both approaches utilize lumped NEG and ion pumping at either end of the chamber. One approach uses no additional pumping over the length of the chamber, relying on thorough cleaning and baking to minimize surface outgassing. The other method uses strips of sintered NEG material in diagonally opposed corners of the chamber top and bottom. The NEG material would be activated by heating

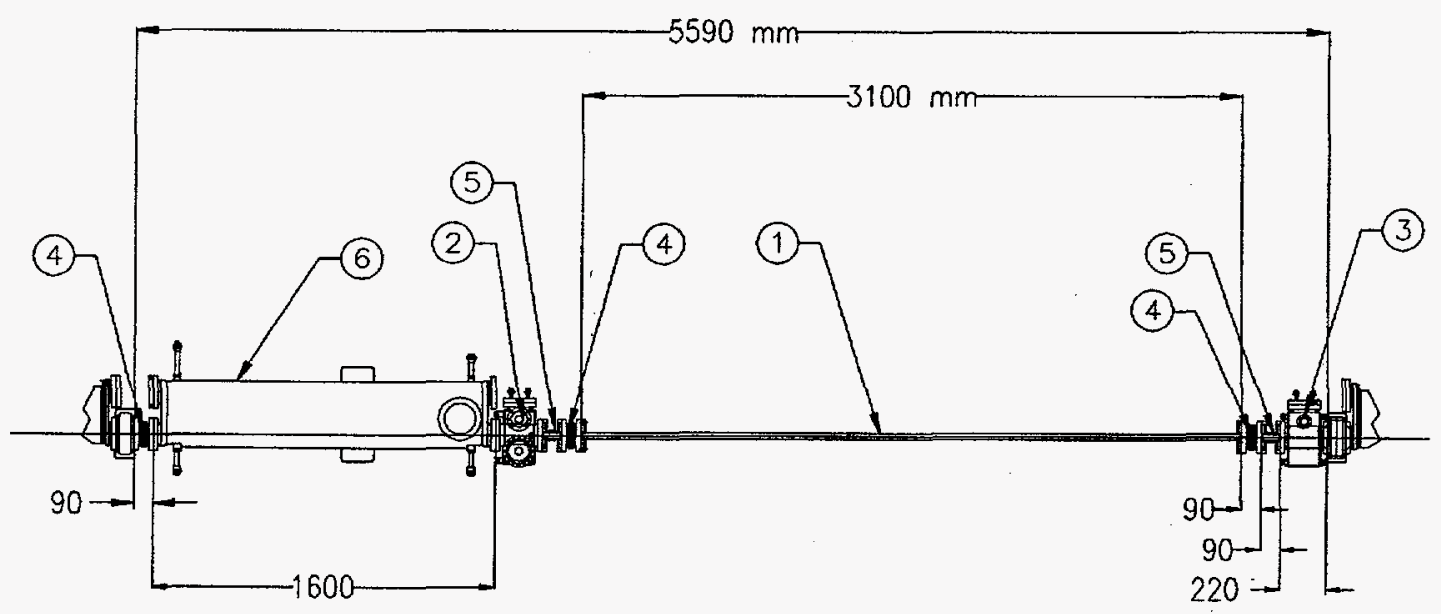

Fig. 4.9 General layout of the APS EMW vacuum chamber: (1) EMW vacuum chamber, (2) upstream end box with transition section, (3) downstream end box with transition section, (4) bellows, (5) BPM insert, (6) short storage-ring vacuum chamber. 
the chamber. Both approaches are complicated by the need to entirely assemble the EMW magnet structures prior to closure of the vacuum system. The system used for in situ heating in either approach must maintain the permanent magnet structures below $35^{\circ} \mathrm{C}$. Activation of the NEG strips requires heating the NEG material to at least $250^{\circ} \mathrm{C}$.
As shown in Figure 4.10, the heating system uses copper plates in contact with the outside of the vacuum chamber. A total of four plates is used, covering most of the chamber length on the top and bottom. A $1 \mathrm{~kW}$ heater is mounted to each copper plate. Contact between the heaters and plates, and between

\section{Magnet Pole}

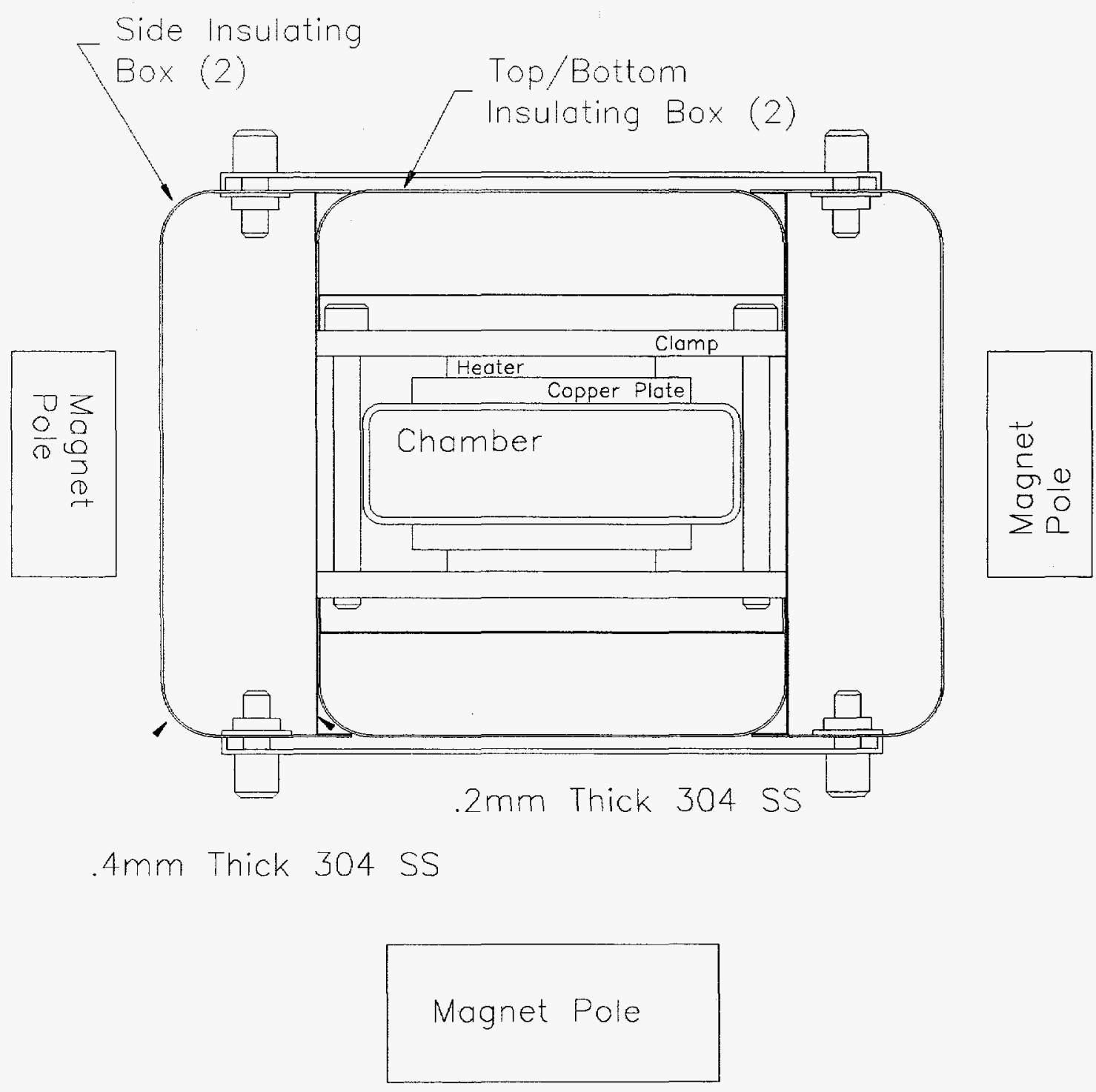

Fig. 4.10 Detail of in situ bakeout heaters for EMW vacuum chamber. 
the plates and the chamber, is maintained with clamps held together with screws. Insulating boxes encapsulate the heating assembly. These boxes are made of thin stainless steel and are filled with layers of ceramic fiber insulation. The insulating boxes are held in place with thin stainless-steel channels, fixed to the side boxes with knurled screws. The entire heating and insulating system can be installed within the EMW structure after the magnet assemblies have been retracted.

While an acceptable pressure for installation had been achieved with the NEG-pumped chamber, it was not clear that the heatactivated NEG material provided a clear benefit. Heating the NEG material from the outside meant that the activated NEGs were subjected to heavy outgassing from the hot chamber, reducing their pumping speed and capacity upon cooling. Thus, the third test focused on the chamber without NEG pumping. The cleaned chamber was assembled, the copper strips were thoroughly cleaned to remove the sulfide/oxide scale, and the heating/insulating system was reassembled. The chamber was heated to $150^{\circ} \mathrm{C}$. The heaters were at $225^{\circ} \mathrm{C}$ and the outside of the insulating box reached a maximum of $61^{\circ} \mathrm{C}$. With ion pumping and activation of the other NEGs, a pressure of $1.7 \times 10^{-10}$ Torr was obtained, better than the level of the NEGpumped chamber. This chamber was used for the EMW installation.

\subsubsection{Development of a Novel Vacuum Sealing Technique}

A common feature of the ID vacuum chambers is the use of bonded aluminum and stainless steel in bimetallic transitions, which allow the joining of stainless-steel and aluminum parts. A roll-bond joint formed with a layer of 304 stainless-steel alloy, a layer of pure aluminum, and a layer of 2219 aluminum alloy, provides a transition from the main chamber body (of 6063 aluminum) to the stainless-steel conflat flanges.

In the process of vacuum testing these chambers, several small vacuum leaks were discovered in the bonds of a few of the chambers. Twenty-six UHV vacuum chambers were fabricated using these transitions, twenty-one for use at the APS, four for the BESSY II project, and one for the ESRF. In these chambers, this sandwich plate was utilized for 52 standard end joints and for 24 circular ports for the BESSY II collaboration. Of the 52 standard end joints, leaks have been detected in five joints. Of the 24 BESSY II ports, leaks were detected in six joints.

To repair these leaks and prepare the chambers for use in an ultrahigh vacuum, high radiation dose environment, a completely new method was tested using high velocity oxygen fuel (HVOF) metal powder spray deposition. An HVOF system accelerates a powder of the selected coating material to supersonic velocities by injecting the powder into a combustion chamber and out through a nozzle with the combustion products. A variety of coating materials can be used including many metals and alloys, as well as ceramics. The high momentum of the particles creates a deposit with high adhesion, fine grain, and low porosity. It is these features that suggested its use for sealing small vacuum leaks. The desired seal must introduce no contaminants into the system, particularly hydrocarbons, must be capable of withstanding repeated bakeouts at $150^{\circ} \mathrm{C}$, and must be immune to radiation damage from $\mathrm{x}$-rays and bremsstrahlung.

To test the feasibility of the process, samples were prepared and sent to vendors to test different coating processes and materials. One 
of the samples was sealed completely by using the HVOF process and $\mathrm{a}_{3} \mathrm{C} / \mathrm{NiCr}$ coating, verifying the potential of the process.

The HVOF process is subject to a great number of variables including surface preparation, powder mixture, particle mesh, feed rates, gas mixture and consumption rates, spray distance and deposition rate, coating thickness, number of coatings, substrate temperature, and environmental conditions, such as humidity. Each of the ID vacuum chambers was sprayed under different conditions, making generalized conclusions difficult if not impossible without increasing the number of samples. Results vary from sealing the leak, to no change, to actually increasing the size of the leak (probably due to thermal effects). Given the proper set of conditions, however, it has been shown that it is possible to seal vacuum leaks in a manner that is completely consistent with ultrahigh vacuum requirements. Further tests are planned to define the proper parameters.

\subsubsection{ID Installation and Commissioning}

The first ID was installed in the storage ring in August 1995, after the installation of the first ID vacuum chamber. This first chamber had an aperture of $12 \mathrm{~mm}$. The very satisfactory initial tests that were run at the time found that the effect of moving the undulator gap on the stored particle beam was smaller than the requirements-in fact, it was only measurable above the noise after statistics had been gathered for many minutes! Harmonics could be seen clearly in the undulator spectrum to over $100 \mathrm{keV}$. The $\mathrm{x}$-ray beam size and angular divergence were also used to determine the emittance of the stored particle beam. These results were reported at the 1995 Synchrotron Radiation Instrumentation
Conference (Cai et al., 1996a, 1996b). The undulator was subsequently removed from the storage ring to allow commissioning of the ring to proceed with the installation of the smaller $(8 \mathrm{~mm})$ aperture ID vacuum chambers.

In the fall of 1995, installations of IDs on the 8-mm-aperture vacuum chambers began. In January 1996, there were three IDs installed. At the end of March 1997, there are a total of 18 IDs installed around the ring (Table 4.1).

Note that there are two undulators installed in Sector 2. The straight sections are long

Table 4.1 IDs Installed on the Storage Ring as of March 1997

\begin{tabular}{|c|c|c|}
\hline Sector & ID installed & $\begin{array}{c}\text { Period length } \\
\text { (mm) }\end{array}$ \\
\hline 1 & undulator & 33 \\
\hline 2 & undulator & 33 \\
\hline 2 & undulator & 55 \\
\hline 3 & undulator & 27 \\
\hline 5 & undulator & 33 \\
\hline 7 & undulator & 33 \\
\hline 8 & undulator & 33 \\
\hline 10 & undulator & 33 \\
\hline 11 & elliptical wiggler & 160 \\
\hline 12 & undulator & 33 \\
\hline 13 & undulator & 33 \\
\hline 14 & wiggler & 85 \\
\hline 17 & undulator & 33 \\
\hline 18 & undulator & 33 \\
\hline 19 & undulator & 33 \\
\hline 20 & undulator & 33 \\
\hline 33 & undulator & 33 \\
\hline 35 & undulator & 18 \\
\hline & & \\
\hline
\end{tabular}


enough to accommodate two of the standardlength (2.4 meter) IDs. Other sectors may install a second $\mathrm{ID}$ in the future if, for example, a different period-length undulator would significantly increase their brilliance in some particular wavelength range. The users close the gap of whichever undulator they want to use at that particular time. Some of the undulators are longer, however, and occupy the entire straight section, such as the elliptical wiggler and the 18-mm-period undulator that is being used by the APS Diagnostics Group to monitor beam quality.

Initially, the gap of an ID was moved only by an ID Group member, only from the Main Control Room, and only with the full knowledge of the accelerator physicist who was operating the storage ring. After the initial testing of the effect of an ID on the stored particle beam showed that the effect was barely measurable, however, permission was routinely given to change the gap as requested by the user, without the need to inform the ring operator. Control of the IDs was then entrusted to the Floor Coordinators, who could move the gaps from computer terminals in their offices on the experimental floor as requested by the users. More recently, users are being given the freedom to control the gap of their ID themselves. As of the run time in March 1997, users in ten sectors now may change their ID gap whenever and however they wish during normal user beam time. For now, Floor Coordinators still open all ID gaps before beam injection, then return the gaps to their previous values once injection is complete.

\subsubsection{Storage Ring Measurements}

Careful measurements of the magnetic field of an ID are made to predict the effect of the ID's magnetic field on the stored particle beam.
The measurements are used to tune the magnetic field, as described above, to minimize undesired effects on the stored beam. The real test of what has been achieved, however, is to install the ID in the storage ring and to measure the actual effect it has on the stored beam. The gap of the ID is cycled between minimum gap and, typically, $45 \mathrm{~mm}$. The global beam position feedback in the storage ring is turned off for these experiments. The variation in the beam position monitor (BPM) readings between the two different gaps is recorded for BPMs all around the ring. Calculations of the closed orbit are then performed in which a kick is added at the position of each end of the particular ID being tested, and the size of the kicks adjusted to fit the observed closed orbit change. These kicks are then used to compute the first and second field integrals. The integrals determined in this way agree with the first and second integrals of the magnetic field through the $\mathrm{D}$ as measured in the APS Magnetic Measurement Facility.

Another important measurement of the effect of an ID on the stored beam has also been carried out. The gap on one ID was moved through its full range of travel while the position of the $\mathrm{x}$-ray beam was observed in a different sector. No movement of the x-ray beam in the second sector was seen, to a sensitivity of 4 microradians. Work is in progress to repeat this measurement with an even higher sensitivity. Note that this is with no active local feedback correction on the particle beam orbit; only global feedback was in operation.

The motion of the $\mathrm{x}$-ray beam due to changes of the gap in the ID producing the beam has also been measured, using a zone plate to image the beam onto a position-sensitive detector. The position of the $\mathrm{x}$-ray beam was found to be constant to within the 
3 microradian sensitivity of that measurement, again with no local active feedback on the particle beam.

\subsubsection{Spectral Performance}

The APS undulator A provides high brilliance $\mathrm{x}$-ray radiation in the $3.2-45 \mathrm{keV}$ spectral energy range. In order to characterize the performance of the undulator, absolute measurements of the undulator brightness as a function of angle and energy have been made. The quality of the undulator magnetic field may be verified by observing the high order harmonics of the undulator radiation. Undulator brightness depends on the particle beam divergence and energy spread.

These measurements may have to be made under conditions of high incident beam power and high incident beam power density. The experimental setup is schematically shown in Fig. 4.11. Upstream of the two spectrometers, a water-cooled conical pinhole with an exit diameter of $0.8 \mathrm{~mm}$ was used to remove a substantial fraction of the undulator power from the beam. Vertical and horizontal watercooled slits define the angular acceptance of

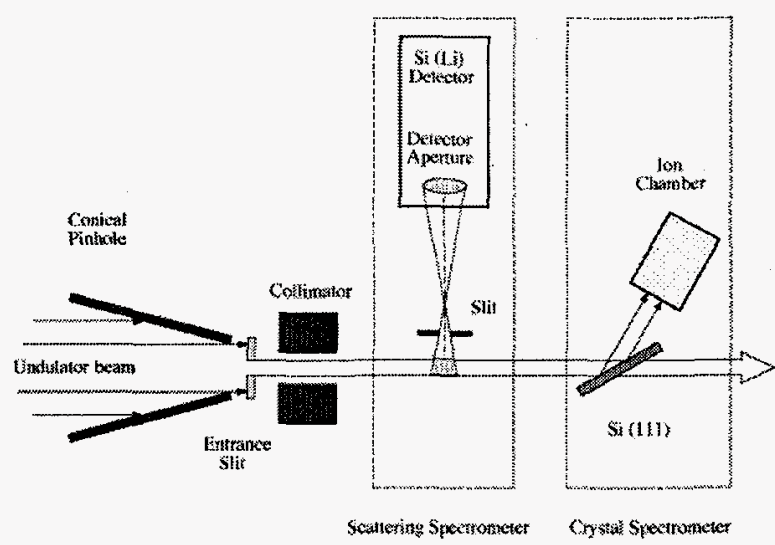

Fig. 4.11 The experimental setup used to measure the spectrum of $x$-rays from an undulator. the incident beam. In order to collimate the bremsstrahlung radiation, a 100 -mm-thick tungsten block with a 5-mm-diameter hole was placed downstream from the slits. The pinhole, slits, and the tungsten collimator were placed in a helium enclosure and were carefully shielded with lead. Two spectrometers were used for absolute flux measurements. The first uses the scattering from a gas whose differential cross section is well known, combined with an energydispersive detector. While the use of an energy-dispersive detector can provide convenient and fast absolute flux and linear polarization measurements, in order to obtain more accurate measurements of the odd undulator harmonics, it may be necessary to use a crystal spectrometer. The crystal spectrometer employs a $\mathrm{Si}$ (111) crystal and an ion chamber detector filled with nitrogen gas. The single-reflection geometry makes spectral measurements quite insensitive to thermal distortion of the crystal because the integrated reflectivity of the crystal can be calculated by dynamical diffraction theory even in the presence of a moderate thermal bump. The efficiency of the ion chamber was determined by calculating the fraction of x-rays absorbed in a $100 \mathrm{~mm}$ active path. The silicon crystal was characterized using surface topography and $\mathrm{x}$-ray diffraction and was found to be a perfect crystal.

A comparison of experimental results versus calculations for on-axis flux tuning curves for the first, third, and fifth harmonics of radiation for undulator $A$ is shown in Fig. 4.12. An aperture of $150 \times 75 \mu \mathrm{m}$ was located $28.9 \mathrm{~m}$ from the source. The typical storage-ring current was $40 \mathrm{~mA}$. There are two calculated curves for the third and fifth harmonics because of different coupling constants during the data collection $(8.5 \%$ for small undulator gaps and $4.6 \%$ for large 


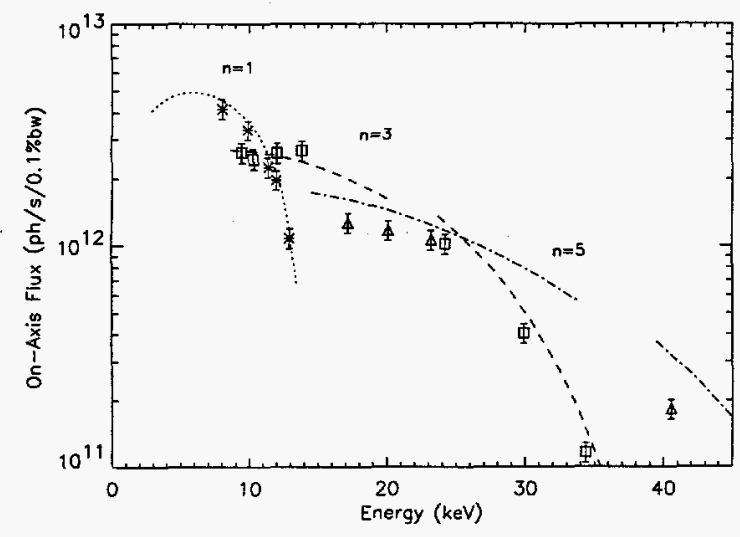

Fig. 4.12 On-axis flux of $x$-rays from an undulator as a function of energy. The first, third, and fifth harmonics are shown. The points are measured results; the lines are the results of calculations. There are two calculated curves for the third and fifth harmonics because the experimental measurements were made at two different times when the particle beam parameters were different.

gaps). Experimental results were normalized to a beam current of $100.0 \mathrm{~mA}$. The error bars are $\pm 10 \%$. The discrepancy between the measurements and the calculations can be attributed to (1) the magnetic field errors of the real device, which cause the intensity of the higher harmonics to be reduced from the ideal, and (2) the error in the emittance measurements, which are used for flux calculations. The anticipated reduction is most easily obtained by numerical simulations using the code UR for the practical case of including the beam emittance, the beam energy spread, and a finite size aperture.

The APS beam emittance was obtained by measuring the vertical and horizontal size and divergence of the undulator $\mathrm{x}$-ray beam. Undulator radiation passes through a watercooled conical pinhole, which reduces the incident power on the cryogenically cooled Si (111) crystal. This horizontally deflecting
Si crystal is vacuum sealed between two $150-\mu \mathrm{m}$-thick beryllium windows. A gold zone plate is used to form an image of the x-ray source. A platinum order sorting aperture was used to increase the contrast in the image. An energy-dispersive $\mathrm{Si}(\mathrm{Li})$ detector was located close to the image plane to measure the $\mathrm{x}$-ray fluorescence generated from a sharp $\mathrm{Ni}$ knife-edge ( $\mathrm{K} \alpha$ emission line) placed in the imaging plane. The beam size was determined from fluorescence intensity profiles measured when the knifeedge was scanned across the beam. The resulting rms source sizes are $300 \pm 25 \mu \mathrm{m}$ in the horizontal direction and $60 \pm 9 \mu \mathrm{m}$ in the vertical. Beam divergence measurements were performed by scanning a slit across the undulator beam in the vertical and horizontal directions, at a monochromator energy that is on the low energy side of the undulator harmonic. This energy is chosen because it substantially reduces the intrinsic undulator divergence contribution. The beam divergence was obtained by fitting the measured beam profile with a calculated one. The fitting gives the result of $25 \pm 2.5 \mu \mathrm{rad}$ for horizontal divergence and $5.3 \pm 0.5 \mu \mathrm{rad}$ for vertical. The corresponding horizontal $(x)$ and vertical (y) emittances (obtained at a current of $20 \mathrm{~mA}$ ) are

$$
\varepsilon_{x}=7.5 \pm 6.0 \times 10^{-9} \mathrm{mrad}
$$

and

$$
\varepsilon_{y}=3.2 \pm 6.0 \times 10^{-10} \mathrm{mrad}
$$

The coupling constant $\chi$, which is the ratio of the vertical to the horizontal emittance, thus becomes $4.3 \pm 1.3 \%$.

The measured on-axis spectral brilliance (solid line), using the crystal spectrometer, and calculated on-axis spectral brilliance (dotted 
line) of the undulator radiation from a $7 \mathrm{GeV}$ electron beam at a gap of $15.8 \mathrm{~mm}(\mathrm{~K}=1.61)$ are shown in Fig. 4.13. The calculation included the measured magnetic field of the undulator, the electron beam emittance, measured at the time of the brilliance measurements, $\left(\varepsilon_{\mathrm{x}}=6.9 \mathrm{~nm}-\mathrm{rad}, \varepsilon_{\mathrm{y}}=0.2 \mathrm{~nm}-\mathrm{rad}\right)$, and the design value for the electron beam energy spread $(0.1 \%)$. The error bars are $\pm 18 \%$, which includes systematic errors for flux and emittance measurements. As is clearly seen from the figure, the measurements are in remarkably good agreement with the calculation. The quality of the undulator magnetic field was verified by observing the high order harmonics of the undulator radiation. More than thirty harmonics can clearly be seen in Fig. 4.14, where the measurements performed at an undulator gap of $11.1 \mathrm{~mm}$ are shown along with the results of calculations that used the measured magnetic field.

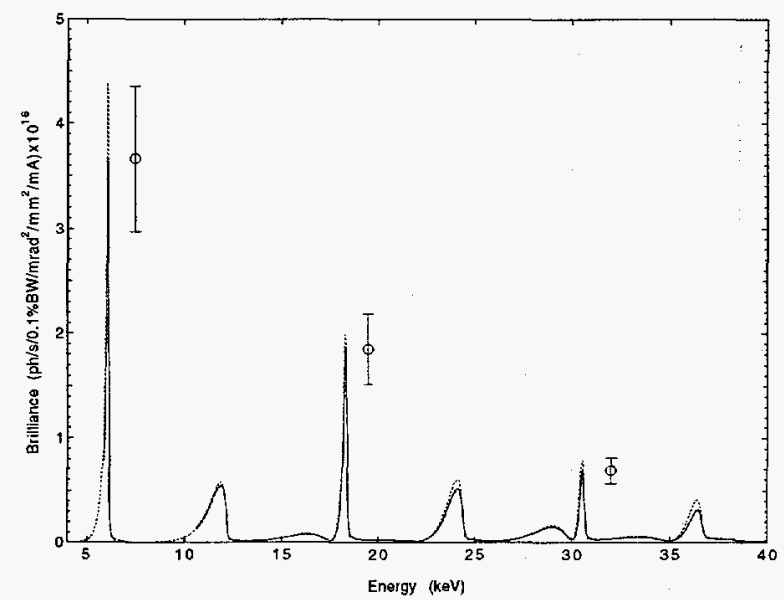

Fig. 4.13 The on-axis spectral brilliance is shown. The solid line was measured using the crystal spectrometer; the dotted line is from a calculation. The calculation included the measured field of the undulator, the measured electron beam emittance, and the design value for the energy spread.

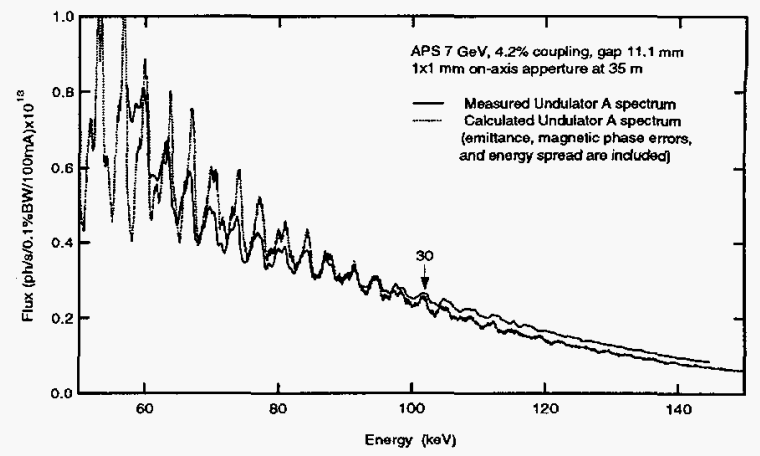

Fig. 4.14 The harmonic structure of the undulator radiation at higher energies. Harmonics as high as the 30th are clearly seen.

\subsubsection{Computer Codes for Simula- tion of Synchrotron Radiation}

Computer simulations of synchrotron radiation are invaluable for characterization of new IDs being installed in the storage ring. Generally, the simulation of the radiation can be divided into two categories-the first being simulation of radiation from an ideal device albeit with a real electron beam, the second being simulation of the real device with the real beam. The simulation of real devices with the real beam is computing intensive but manageable with today's high speed workstations and personal computers with results obtained in typically one day of computing time. Computer codes for both categories have been developed in collaborative efforts at the APS, and a few results of these codes will be presented here.

Quite often it is not enough to have access to a computer code that, in principle, will solve a particular need because the code is difficult to use - either because of poor documentation or because of the complicated nature of the code. 
In this regard, the use of graphical user interfaces for entering data and for displaying results has become important. A suite of programs for modeling radiation characteristics of ideal devices (real electron beams) and the interaction with optical elements has been developed and assembled into a package called XOP (x-ray optics utilities) using a common graphical user interface. This interface largely simplifies the execution of the codes and may be run without any prior knowledge of how to run a particular program.

\subsubsection{XOP: A Graphical User Interface for X-ray Optics Utilities}

The XOP interface contains a suite of programs of general interest to the synchrotron radiation community (Dejus and Sanchez del Rio, 1996). They are easy to use, fast, and the results are visualized and analyzed using the provided plotting interface-all tasks are performed without leaving the interface. The programs have been naturally divided into two general categories (Fig. 4.15a)-computer codes for generation of synchrotron radiation properties of undulators, wigglers and bending magnets, and codes for computation of optical constants, photon cross sections, mirror reflectivities, filter transmissions, crystal diffraction profiles, and multilayer reflectivities.

The interface, which was written in the IDL (interactive data language) programming language, runs on workstations for most flavors of the UNIX operating system including Sun Solaris, HP-UNIX and Digital UNIX. It is also available for PCs running Windows-95 and Linux. Other operating systems will be available as demand changes.
The package is stand alone, no commercial license is needed, and is available free of charge to non-profit organizations.

Figures $4.15 \mathrm{~b}$ and $4.15 \mathrm{c}$ show the pop-up menu (Xus) for the code US (undulator spectrum) with the accompanying input menu for entering data and for setting parameters and options. In this example, the calculation of the flux spectrum through an aperture was chosen (size of aperture was chosen to approximately cover the central cone of radiation). The results are visualized and analyzed using the built-in plot interface Xplot.

The calculated emitted $\mathrm{x}$-ray spatial distribution at select energies (near harmonic energies) has been verified experimentally. The single electron radiation pattern shown in Fig. 4.16a was calculated using the Xus interface and broadened with the introduction of the beam emittance of the stored beam. The broadened profile was successfully matched with the measured profile near the third harmonic energy by fitting the size and divergence parameters of the beam. Fig. 4.16b shows the result of the best fit and the measured beam intensity profiles (vertical only). The code was further verified experimentally by measuring the tuning curves of the absolute spectral flux for the first three odd harmonics of radiation (see section 4.1 .10 on spectral performance).

\subsubsection{UR: Computer Code for Simulation of Real IDs with Real Beams}

The computer code UR (undulator radiation) has proven instrumental when predicting the spectral performance of the APS insertion 

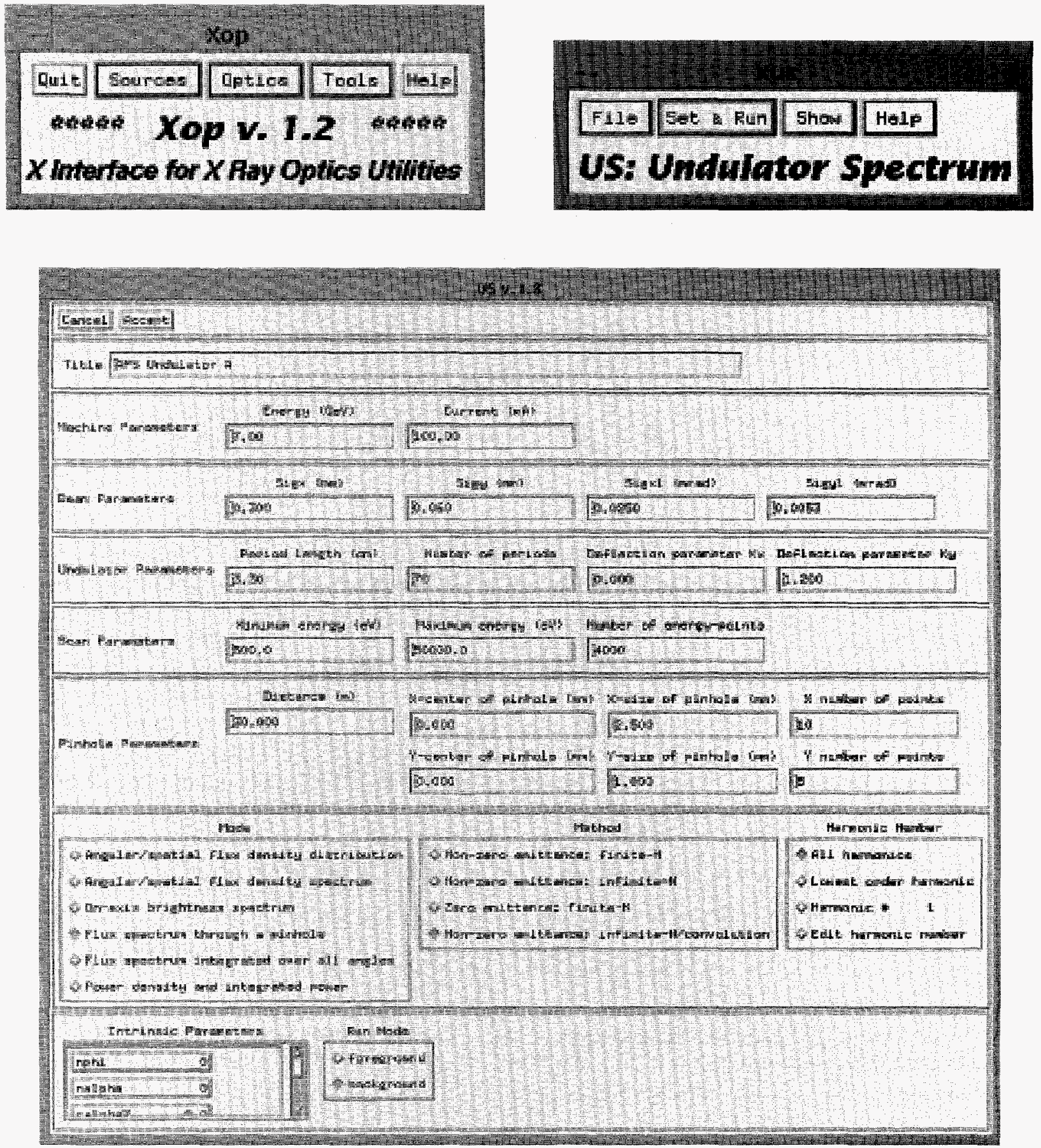

Fig. 4.15 (a) (upper left) The main menu of XOP with separate buttons for sources and optics utilities. (b) (upper right) Pop-up menu for the code US (Undulator Spectrum). (c) Input menu for the code US. 

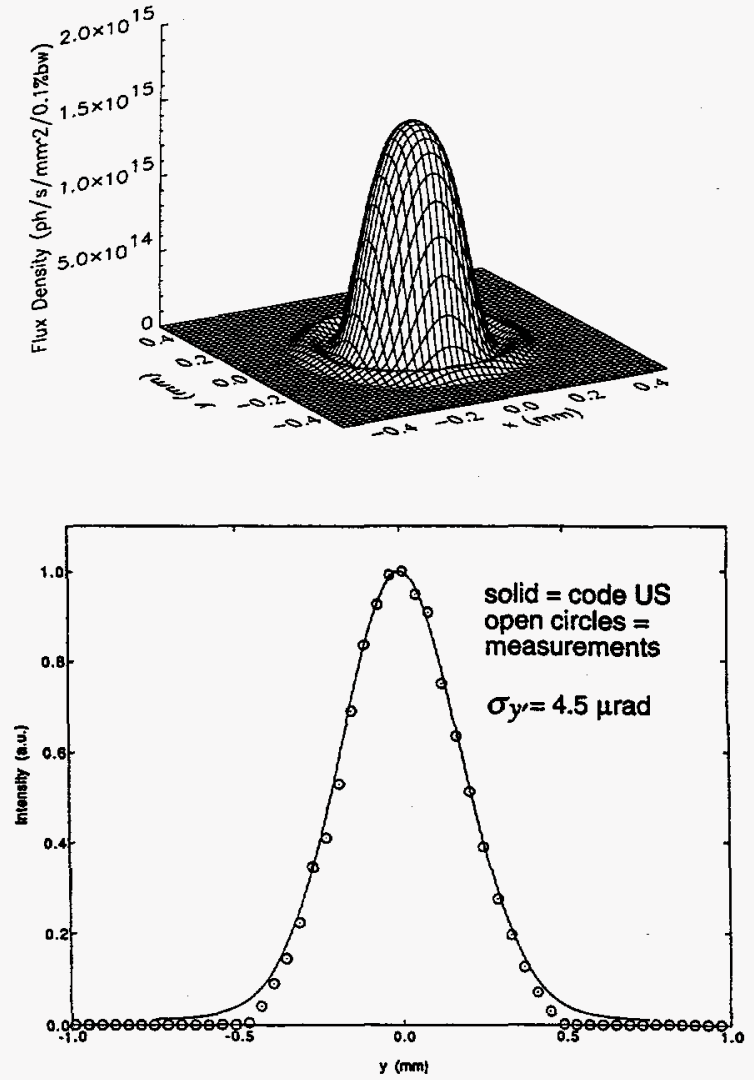

Fig. 4.16 (a) (top): Single electron radiation pattern at $32.8 \mathrm{~m}$ near the third harmonic energy (18.4 keV) for undulator $A(K=1.61)$. (b) (bottom): Calculated and measured vertical beam intensity profiles of radiation at $18.4 \mathrm{keV}$ for undulator $A(K=1.61)$.

device on an absolute level. The computer code solves numerically the equations of motion (3-D) for an arbitrary magnetic field and calculates the radiation field at each time step as the particles are tracked through the device. The real beam emittance and beam energy spread and the actual magnetic field of the device are typically used in the calculations (Dejus and Luccio, 1994).

Experimental characterization of undulator A has been performed not only at low photon energies but also at high energies ( $>50 \mathrm{keV}$ ) and comparisons of the measured and calculated spectral flux have been made. The measured and calculated flux through an aperture $(1 \times 1 \mathrm{~mm})$ located $35 \mathrm{~m}$ from the source for an undulator gap setting of $15.5 \mathrm{~mm}(\mathrm{~K}=1.63)$ is shown in Fig. 4.17. The 11th harmonic is seen at $66 \mathrm{keV}$, and its shape and structure has been influenced largely by the non-perfect magnetic field in addition to the effect of the beam emittance and beam energy spread. All three effects make the high harmonics show a complicated pattern-it should be noted that the code is able to predict all harmonics (up to number 29 at $170 \mathrm{keV}$ ) including the detailed substructure of the harmonics.

\subsubsection{Radiation Exposure of the IDs}

$\mathrm{NdFeB}$ magnets are known to be sensitive to radiation damage, and some work has been done in the past, by others, to determine the radiation susceptibility of the $\mathrm{NdFeB}$. Much of the work that has been done, however, is not directly applicable to ID magnets because the spectrum of radiation used in the study or its makeup (e.g., neutrons as opposed to electrons or photons) is not the same as will

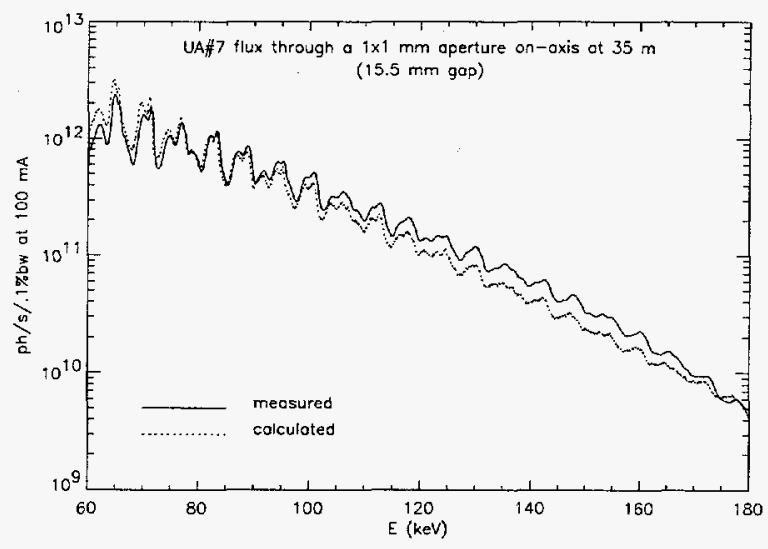

Fig. 4.17 Comparison of measured and calculated spectra for undulator $A(K=1.63)$. 
be present in a storage ring. Also, $\mathrm{Nd}$ magnet technology has developed rapidly in recent years so that the magnet material used in the APS IDs was not available eight years ago. The effect of stabilization of the magnets is unclear, and there may be variation in radiation sensitivity between magnets from different vendors as well (see Chavanne et al., 1996).

There is no doubt, however, that the radiation exposures received by ID magnets are sufficient to cause demagnetization. This has been shown at ESRF by an ID that showed partial demagnetization after being installed for a year (see Colomp and Brauer, 1993). The best estimate for the total dose received by that $\mathrm{ID}$ is 5.1 to $6.8 \mathrm{Mrad}$ at the first magnet. At the upstream end of the device, about $7 \%$ of the peak field was lost due to demagnetization.

Not all the radiation to which an $\mathrm{ID}$ could be exposed will contribute towards demagnetization, however. Experiments have been reported (Okuda et al., 1994) in which magnets were irradiated with $17 \mathrm{MeV}$ electron beams and $1.17 \mathrm{MeV}{ }^{60} \mathrm{Co} \gamma$-rays. Whether the energy quanta are electrons or photons probably does not matter, but the energy of the quanta does make a difference. No demagnetization was seen after exposure to $280 \mathrm{Mrad}$ of $1.17 \mathrm{MeV} \gamma$ 's, but there was a 9\% flux loss for the $\mathrm{NdFeB}$. exposed to $260 \mathrm{Mrad}$ of $17 \mathrm{MeV}$ electrons. The radiation-demagnetized magnets were subsequently remagnetized to essentially full strength.

Clearly, then, one needs to know the spectrum of radiation to which the magnets are exposed in order to predict the likelihood of damage.
An experiment was carried out at the APS to determine whether the dose rates being measured at the IDs using film dosimeters and thermoluminescent dosimeters (TLDs) were from high or low energy quanta. A multilayered sandwich of alternating $\mathrm{Pb}$ and film dosimeters was placed so the dosimeters were approximately $30 \mathrm{~mm}$ directly above the positron beam, as shown in Fig. 4.18. The dose as a function of depth of $\mathrm{Pb}$ was measured, and the results are shown in Fig. 4.19a. For comparison, the absorbed energy as a function of depth in $\mathrm{Pb}$ due to a $6.3 \mathrm{GeV}$ cascade is shown in Fig. $4.19 \mathrm{~b}$ (data from Bathow et al., 1967). The depth at which the peak dose occurs is a function of the energy of the incident quanta. The similarity between these curves suggests that $7 \mathrm{GeV}$ quanta made up a large fraction of the incident radiation in the APS test. This radiation is high enough in energy to demagnetize the magnets.

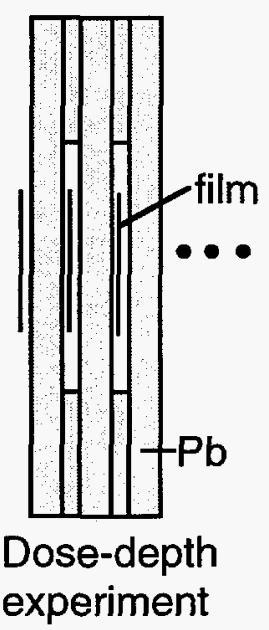

Fig. 4.18 Schematic of part of the experimental setup used for the dose-depth experiments. The setup consists of a 16-to-32 layer sandwich of film dosimeters between Pb plates. 


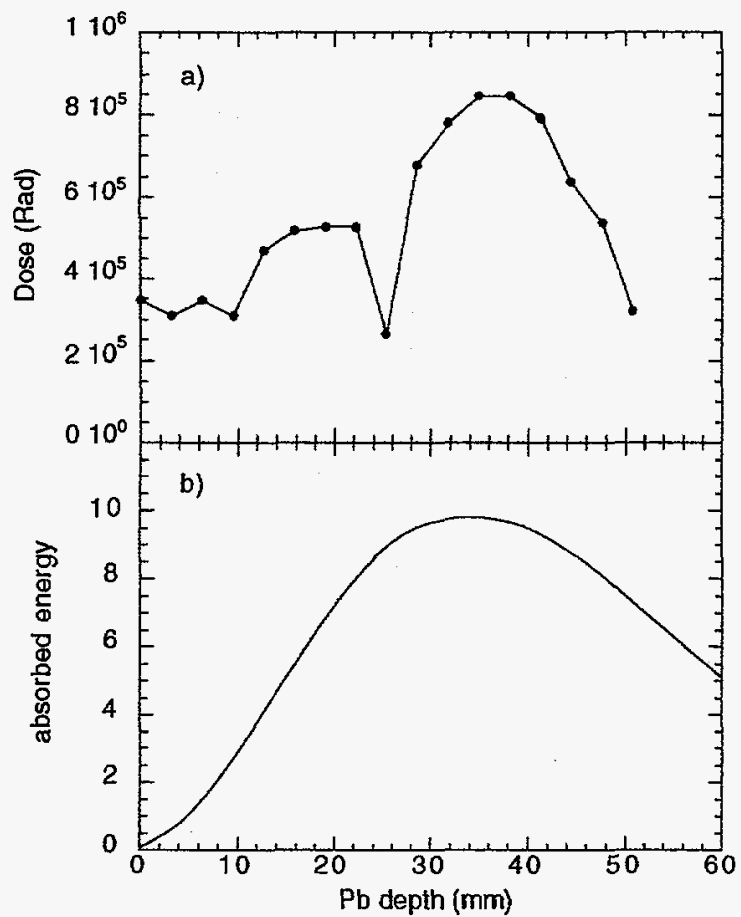

Fig. 4.19 (a) The results of the dose-depth experiment are shown. Each point is the reading from one film dosimeter. (b) For comparison, the published results (Bathow et al., 1967) of a simulation of the absorbed energy as a function of depth in $\mathrm{Pb}$ due to incident $6.3 \mathrm{GeV}$ quanta were graphed.

The depth-dose experiment was repeated during the subsequent run. In that experiment, the peak dose occurred at a shallower depth, suggesting that some of the cascade had already taken place before the radiation reached the experiment. It may be that the spectrum of the dose reaching the $\mathrm{ID}$ varies strongly with events or beam characteristics that are specific to the particular run. More experiments will be conducted to further characterize the exposure.

Dose measurements made during the first running period with IDs installed and with the smaller gap vacuum chamber alerted us to the need to provide some radiation shielding for the IDs. During much of that run, injection efficiency was quite low and for long periods of time beam was being injected even though it wasn't being successfully stored. When the dosimeters were removed and read out, two of the three installed IDs were found to have been exposed to extremely high doses. The response of the TLDs used flattens out at exposures over about $300 \mathrm{Krad}$, so it is difficult to know the actual dose to within a factor of 2, but it is estimated to have been as high as $5 \mathrm{Mrad}$ on one ID and $1 \mathrm{Mrad}$ on the other. The gap on the third ID had been kept open during the entire run, so its dose was only $52 \mathrm{Krad}$ on the first pole. However, a second dosimeter that was placed to measure what the dose would have been for that ID if it had been at minimum gap for the entire run gave an estimated dose of $3 \mathrm{Mrad}$. The magnetic field of the undulators that have received the highest doses was rechecked, most recently in March 1997, and no demagnetization was found.

After these surprisingly high dose rates and knowing the experience at ESRF, it was decided that measures to reduce the exposures encountered by the installed IDs were warranted. As a result, IDs are now installed in the downstream part of the ID straight section whenever possible, and $\mathrm{Pb}$ shielding is installed in the open space upstream of the ID.

The $\mathrm{Pb}$ shielding that has been installed is more than 30 radiation lengths thick and should reduce the radiation levels to something too small to measure. The measured effect of the $\mathrm{Pb}$ gives us insights into the spatial distribution of the radiation. Fig. 4.20 shows the dose measured during a 3.5-week run by film dosimeters laid flat on the upper 


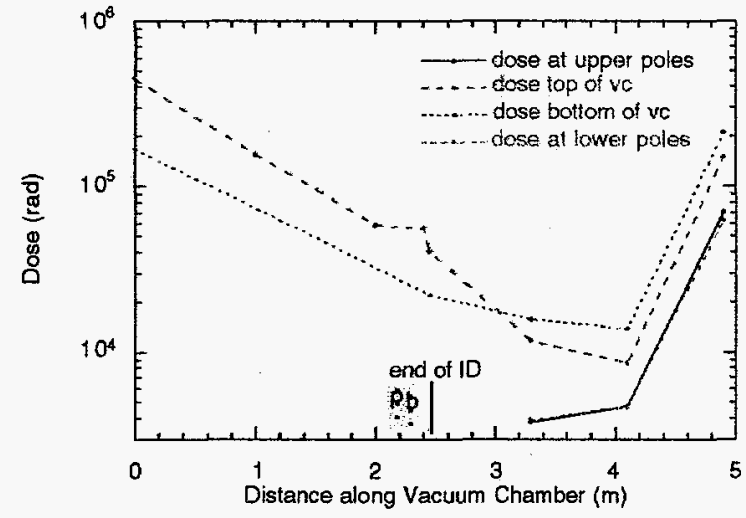

Fig 4.20 Doses measured using film dosimeters that were placed flat on the top or bottom of a 10-mm-outside-dimension ID vacuum chamber. Dosimeters were also. placed on the faces of the pole of the ID, which was kept at $100 \mathrm{~mm}$ gap throughout the 3.5-week run. The position of the Pb shielding upstream of the ID is shown. The dose at the upstream end of the ID (marked on the graph) was too small to measure by this technique $(<3000$ Rad).

and lower faces of the (10-mm-outside dimension) vacuum chamber and laid flat on the pole faces of the ID. This ID was kept at $100 \mathrm{~mm}$ gap throughout the run. The position of the $\mathrm{Pb}$ shielding is marked on the graph; note that it has no effect on the distribution of doses measured at the face of the vacuum chamber, despite the fact that there is no gap between the $\mathrm{Pb}$ shielding and the vacuum chamber. The $\mathrm{Pb}$ does dramatically reduce the dose at the upstream face of the first pole where the unshielded doses were highest; however, that point is not shown on the graph because the dose there was too small to measure. The $\mathrm{Pb}$ shielding has essentially no effect on the dose at the downstream end of the ID. This shows that while there is radiation traveling through the air above the vacuum chamber, there is also a nonnegligible amount of radiation that will reach the magnets of an ID at closed gap by traveling through the vacuum chamber. So although the shielding dramatically reduces the radiation dose at the upstream end of the ID, where it would otherwise be highest, it has essentially no effect on the lower dose levels at the downstream end.

Another means that is used to reduce the radiation dose to the magnets is to leave the $I D$ gaps open when the IDs are not in use and, more specifically, to open the gaps for injection. Figure 4.21 shows the total dose measured during a 6-week running period at the first poles of each of the installed IDs. Dosimeters were also mounted immediately upstream of the first pole of each ID, at the same distance from the vacuum chamber as the dosimeter on the first pole would be if the

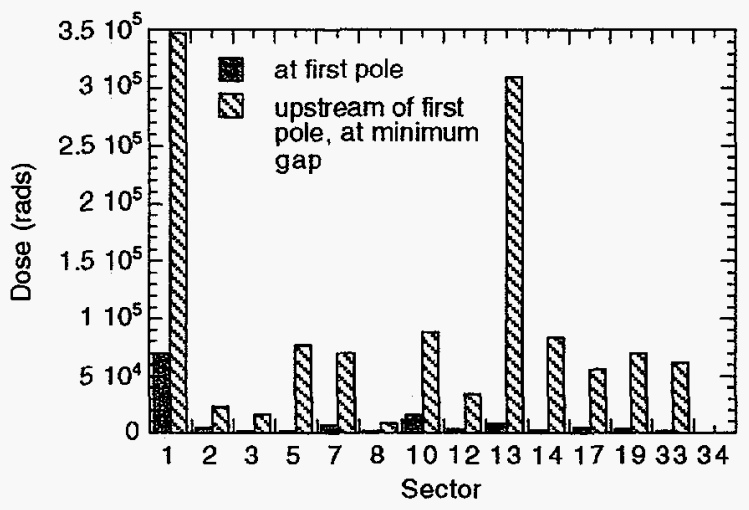

Fig. 4.21 Dosimeters were placed on the upstream face of the first pole of each of the IDs. There were also dosimeters placed on the vacuum chamber immediately upstream of the ID, at the same distance from the particle beam as the dosimeters on the poles would be if the ID were at minimum gap. Thus, the difference between the two bars in each sector shows the radiation exposure the ID was spared by not being at closed gap during the entire run. 
ID were at minimum gap. Thus, the difference in dose rates indicates the amount of dose the ID was spared by having the gap open when the ID was not being used.

While these measures that have been taken to reduce the exposure of the IDs have been effective, they are not the final answer. One reason why is that not all IDs can be protected by $\mathrm{Pb}$ shielding because there isn't always enough space for the shielding. Another reason is that an eventual goal in the operation of the storage ring is to go to a "top-off" mode in which small amounts of beam would be injected frequently so that the beam current would be kept nearly constant. That way, the users would have a beam with constant brilliance. These frequent injections would need to be carried out with the ID gaps closed. In order to be confident that this would not be putting the IDs at risk, a better understanding of the origin of the dose is needed-it could be mainly coming from beam that is lost during injection, it could be a steady dose from the entire time the beam is operating, or it could be from particular beam-loss events. A means of monitoring radiation levels in real time to better understand the origin of the radiation is being sought. A better understanding of the causes of demagnetization and the dose at which demagnetization should be anticipated may allow us to run the storage ring in the way that best suits all the users, without risking damage to an ID and the resulting down time for the user of that ID.

\subsubsection{Elliptical Multipole Wiggler}

In addition to the standard planar IDs, there is also an elliptical wiggler installed in the ring now. This device was built jointly by the APS and the Budker Institute of Nuclear Physics in Novosibirsk. It has planar arrays of magnets and poles to produce the vertical magnetic field component, just as the standard IDs do. The weaker horizontal field is produced by electromagnets. The device has a $16 \mathrm{~cm}$ period. The total number of poles is 36 for the electromagnets and 37 for the permanent magnet array. Thirty-two of the poles in each direction are full field. The user deemed it important to have an even number of full field permanent magnet poles so that the spatial distribution of radiation from the wiggler would be, in principle, symmetric above and below the beam axis. An even number of fullfield electromagnet poles was expected to simplify the tuning of the field, because the demand for horizontal field quality is higher. The horizontal electromagnet field is designed to operate at a frequency of $10 \mathrm{~Hz}$, at a peak current of $1 \mathrm{kA}$. This current produces a peak horizontal field of $997 \mathrm{G}$, or $\mathrm{K}_{\mathrm{x}}=1.5$. The peak vertical field is $9826 \mathrm{G}$ at the minimum gap of $24 \mathrm{~mm}$, for $\mathrm{K}_{\mathrm{y}}=14.7$.

This wiggler is now installed in the storage ring, and experiments are beginning to measure the degree of circular polarization using a magnetic Compton scattering experiment. The experiment is being performed at $47 \mathrm{keV}, 65 \mathrm{keV}, 86 \mathrm{keV}, 115 \mathrm{keV}$ and $150 \mathrm{keV}$. At each energy, measurements were done for the following $\mathrm{K}_{\mathrm{X}}$ values : $1.3,1.0$, 0.8 and 0 (i.e., no horizontal magnetic field applied). The exception was at $86 \mathrm{keV}$, where the polarity was varied. Helicity switching was observed as expected. 


\subsection{Beamline Engineering}

\subsubsection{Introduction}

The objective of the beamline engineering effort is to design and build all the beamline FE components and to develop standard beamline components that would be of use to all APS CATs. Towards this objective, the team is built around the following expertise:

- knowledge of heat transfer engineering to handle high heat flux from IDs on various mechanical components of the beamline FEs (described in Chapter 2) and beamlines,

- fabrication of components using modern composite materials with the development of suitable bonding, brazing, and welding technologies, and

- precision engineering required in the design of support stages and manipulators for standard beamline components and optics.

The design and construction of these components have now been completed, and many of the beamline FEs and standard components are now installed. The details of this work can be found in various publications (Kuzay, 1993; Shu \& Kuzay, 1994; Shu et al., 1992a; Shu et al., 1995).

During the past year, major effort has been devoted to evaluation of the performance of these mechanical components of the beamlines. In addition, we have conducted special experiments to test the performance limits of some of the critical elements of both the beamline FEs located behind the shield wall of the storage ring and the user beamlines installed on the experiment hall floor. The performance to date of some of these components and the ongoing $R \& D$ in support of the APS operations are summarized here.

Unless otherwise noted, the material presented here describes only the ID beamlines, as their designs are the most challenging and have the most stringent thermo-structural specifications.

\subsubsection{Performance Review of the Important FE and Beamline Components}

\section{Vacuum Breach Tests in APS Beamline FEs}

Measurement of the propagation of a pressure front under simulated $\mathrm{FE}$ vacuum-breach conditions has shown that the engineering of the apertures in the beamline FEs provides adequate vacuum delay to protect the storagering vacuum. The data for the ID beamline FEs were taken on the ID FE prototype (1:1 scale) (Liu et al., 1995); while the data for the BM beamline FEs were collected on 3-BM during operations.

In performing the vacuum-breach tests on the FE prototype, the simulation represented an equivalent of a $6 \mathrm{~mm}$ hole to the atmosphere in a 1-mm-thick window. Four ion gauges (IG1IG4) were placed in the FE for measurement (see Fig. 4.22). Under such a severe vacuum breach, the pressure wave took $48 \mathrm{~ms}$ to reach IG1, $80 \mathrm{~ms}$ to IG2, and $110 \mathrm{~ms}$ to IG3 (Fig. 4.22a). Because the fast valve closed in 

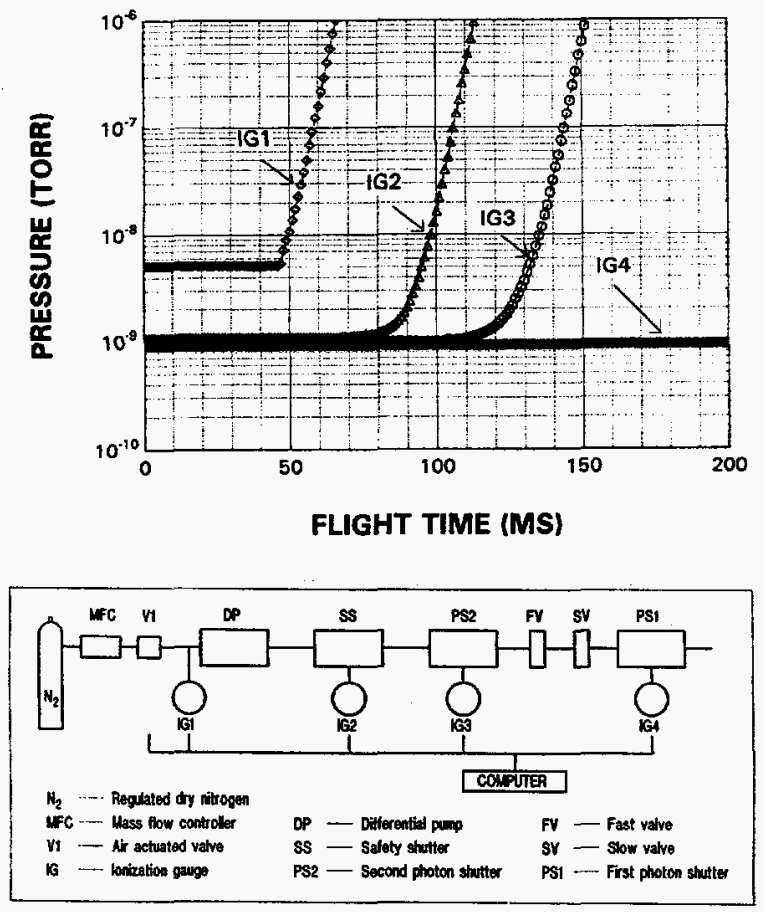

Fig. 4.22 ID FE vacuum-breach tests. (a) Time (in $\mathrm{ms}$ ) for the pressure wave to reach the ion gauges. (b) Schematic of the location of the ion gauges (IGI-IG4).

$6.7 \mathrm{~ms}$, IG4 never experienced the pressure wave. Thus even with a large vacuum breach in a typical ID beamline, the FE has up to $50 \mathrm{~ms}$ delay time for proper closure of the vacuum valves to isolate the strickened $\mathrm{FE}$ from the storage-ring vacuum.

To date the only in situ FE vacuum-breach incident under real user beam operation conditions occurred on beamline 5-ID, which had a windowless configuration. As the result of a miscalculated beam steering request, the undulator radiation beam struck a bellows, which caused substantial outgassing and damage to the bellows. The resulting deterioration in the vacuum was sensed by the cold cathode gauge, which initiated proper EPS actions by closing the $\mathrm{FE}$ valves and isolating the FE. The FE recovered normally through subsequent pumping after the damaged bellows were replaced.

In situ vacuum-breach tests on the 3-BM FE were conducted in a fashion similar to those done on the ID FE prototype. To summarize, in the worst-case leak tests, a shock wave was generated by bleeding nitrogen into the FE at 50 psi for 3 seconds. The ion gauge by the safety shutter registered a $60 \mathrm{~ms}$ time delay, and the ion gauge by the first photon shutter (PS1) registered $120 \mathrm{~ms}$ delay time (vacuum valve closure was disabled). The measured vacuum delays are certainly long enough to activate the $F E$ vacuum valves and in turn protect the vacuum conditions in the storage ring.

\section{Photon shutter tests}

The ID FE components have been designed to handle radiation beams from 2.4-m-long undulators when the storage ring is operated with a maximum of $100 \mathrm{~mA}$ of particle beam. In the FE, the most challenging components to design are the photon shutters. A series of tests were arranged to assess the performance limits of the user-operated photon shutter, PS2, at highest available stored beam currents. Because the maximum stored beam current is $100 \mathrm{~mA}$ during current operations, to simulate higher current operations, the incident angle of undulator beam with respect to the PS2 surface was increased to deliver higher heat flux on the shutter blade.

The beam defining mask associated with the windowless configuration of the FE allowed a full $5.3 \mathrm{~kW}$ of power from the undulator (gap of $11 \mathrm{~mm}$ and stored beam current of $100 \mathrm{~mA}$ ) to impinge on the photon shutter during these tests. In order to increase the heat flux on the 
shutter blade (located at $26 \mathrm{~m}$ from the source in this experiment), the blade was tilted to $6.75^{\circ}$ with respect to the undulator beam. This generated a measured power of $4.3 \mathrm{~kW}$ at $100 \mathrm{~mA}$ or a maximum heat flux density of $27.5 \mathrm{~W} / \mathrm{mm}^{2}$ on the shutter surface.

The maximum temperature registered on the blade at a depth of $2 \mathrm{~mm}$ and approximately at the center of the beam footprint was about $340^{\circ} \mathrm{C}$. While on the sides of the blade, temperatures of 90 and $70^{\circ} \mathrm{C}$ were measured. The measured $340^{\circ} \mathrm{C}$ inside the shutter blade corresponds to a $460^{\circ} \mathrm{C}$ surface temperature. The maximum measured flow enthalpy, as shown in Fig. 4.23, indicates that during the tests the power absorbed by the shutter is $4.1 \mathrm{~kW}$ at $11 \mathrm{~mm}$ gap and at $97 \mathrm{~mA}$ current (or $4.3 \mathrm{~kW}$ at $100 \mathrm{~mA}$ ). This represents about $70 \%$ of the $5.3 \mathrm{~kW}$ full power from the beam at $11 \mathrm{~mm}$ gap and at $100 \mathrm{~mA}$. These measurements agree closely with the pretest analysis using a finite element analysis code (ANSYS).

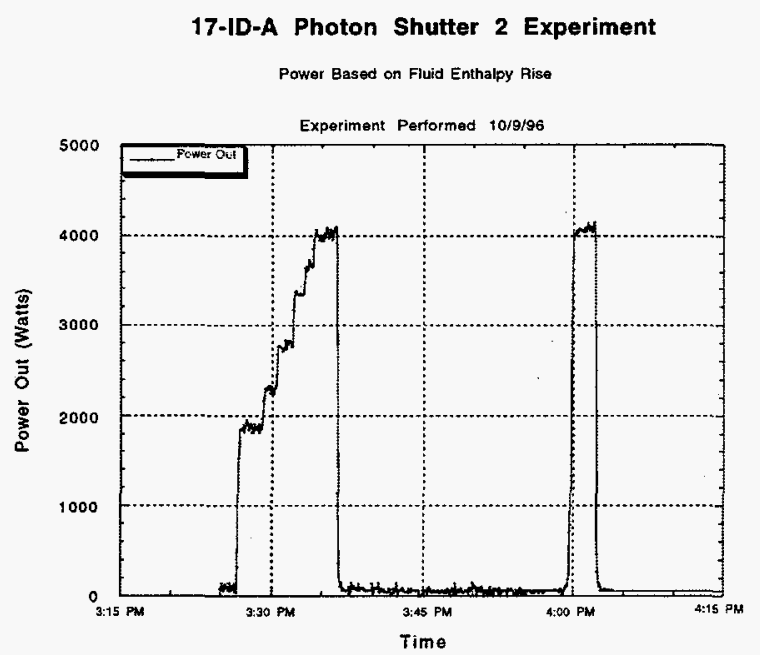

Fig. 4.23 Power absorbed by PS2 based on fluid enthalpy rise.
It is interesting to evaluate the performance of PS2 under its normal operational conditions, viz., its location at $20.1 \mathrm{~m}$ with a inclination angle of $2^{\circ}$. The power density handled in this configuration is about $13 \mathrm{~W} / \mathrm{mm}^{2}$, which is well below the maximum power handled in the tests at $27.5 \mathrm{~W} / \mathrm{mm}^{2}$.

The maximum current limit for the operation of PS2 can also be extrapolated from these studies. Figure 4.24 shows that the experimentally deduced $460^{\circ} \mathrm{C}$ surface temperature is reached at about $190 \mathrm{~mA}$ beam current at $11 \mathrm{~mm}$ gap and with PS2 located at $20.1 \mathrm{~m}$ from the source. The analysis showed that the coolant wall had reached $210^{\circ} \mathrm{C}$, which would

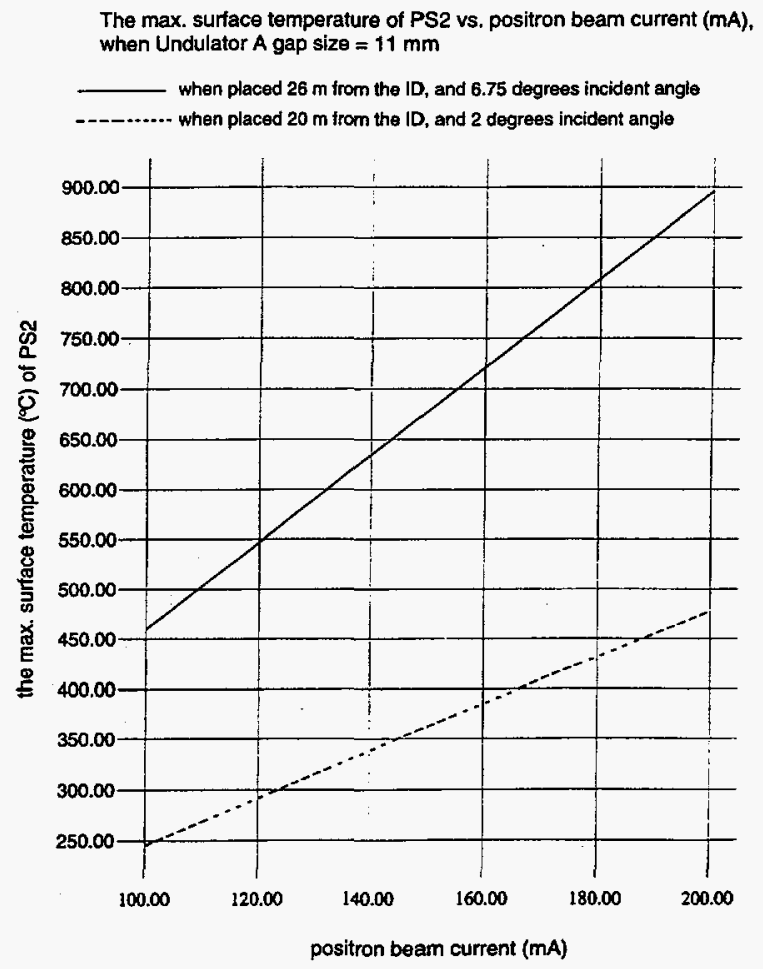

Fig. 4.24 Maximum surface temperature of PS2 versus positron beam current (with undulator $A$ gap size $=11 \mathrm{~mm}$ ). Solid line PS2 is $26 \mathrm{~m}$ from the ID at an incident angle of $6.75^{\circ}$. Dotted line - PS2 is $20 \mathrm{~m}$ from the $I D$ at an incident angle of $2^{\circ}$. 
induce severe boiling. The shutter surface and/or the Glidcop/OFHC copper interface are most likely to experience fatigue under these operating conditions because of extremely high localized thermal stresses. The shutter can tolerate such conditions on a transient or infrequent basis but not on an operational design basis. At about $150 \mathrm{~mA}$, the coolant wall temperature will be about $170^{\circ} \mathrm{C}$, a value beyond the saturation temperature of the coolant water at the prevailing pressures. This sets the limit for PS2 operation to be about 150-160 mA stored beam current with undulator $A$ at a gap of $11 \mathrm{~mm}$.

Photon shutter 1, with its longer mesh length and, thus, much higher pressure drop, will incur boiling of the coolant even at lower stored beam currents. Fortunately, PS1 operational requirements do not demand prolonged exposure to the undulator power densities.

The need for further research into designing FE components that can handle undulator beams with currents higher than $150 \mathrm{~mA}$ is critical and will be the focus during the next few years.

\section{Commissioning Filter and Window Assembly for the APS ID Beamlines}

Although the APS undulator FEs are designed for windowless operation, a special window assembly is used during the commissioning phase of the beamlines until sufficient operational experience is gained with the powerful beam from undulator $A$. This assembly is called a "commissioning window." Due to the high total power and power density, the filter/window assembly was designed to guarantee longevity and good performance (Kuzay et al., 1996; Shu \& Kuzay, 1996). A schematic of the commissioning window is shown in Fig. 4.25. It consists of a $300-\mu \mathrm{m}$-thick filter block (made of graphite), a water-cooled fixed mask (made of Glidcop), a multifunctional diamond disk (made of $160-\mu \mathrm{m}$-thick chemical vapor deposition [CVD] diamond), and a set of windows (made of $250-\mu \mathrm{m}$-thick beryllium). The four blocks of the assembly, which are individually water cooled, are made to be discrete blocks aligned and fastened together instead of an integrated unit. This will allow separation of the fixed mask with its CVD diamond disk so that it can be eventually used on FEs in a windowless configuration with a differential pump. The CVD diamond disk is $160 \mu \mathrm{m}$ thick and $25 \mu \mathrm{mm}$ in diameter and works as power filter to protect the $\mathrm{Be}$ window. It can also act as a contamination barrier to stop backstreaming of heavy hydrocarbons upstream from the beamline side if used in conjunction with the windowless configuration.

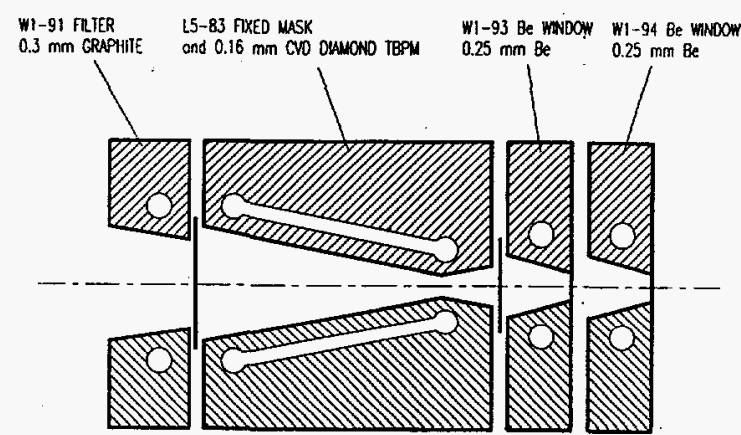

APS W1-90 ID FE COMMISSIONING WINDOW ASSEMBLY

Fig. 4.25 Schematic of the commissioning window assembly for ID beamlines 
For the users, the beam throughput of the commissioning window is of utmost interest. Photon transmission calculations indicate excellent transmission of radiation above about $6 \mathrm{keV}$.

Although, the commissioning windows are currently used on many undulator beamlines, only a limited amount of detailed testing has been performed. The studies have focused on the temperature measurements at the graphite filter and diamond disk with an infrared (IR) camera. These measurements are done at different undulator gaps and different stored particle beam currents. The measurements have been compared in detail with the analytical predictions. In Table 4.2, a measured set has been compared with calculations using different values for effective thermal conductivity.

It is seen from the table that agreement between the measured and predicted values is rather good. However measurements taken the same way about six months later showed much higher temperatures. At closed gap and
$98 \mathrm{~mA}$, a surface temperature higher than $1400^{\circ} \mathrm{C}$ was indicated by the IR camera. The pyrolitic graphite filter was subsequently removed and examined under microscope, which revealed a number of planar cracks in the filter around the beam footprint area that could reduce the conductive heat transfer process. Further investigations are planned in the coming year.

\section{X-ray Beam Position Monitors}

The design of the $\mathrm{x}$-ray beam position monitors (XBPMs) at a third-generation synchrotron source is quite challenging. They must withstand the high thermal load (up to 600 watts $/ \mathrm{mm}^{2}$ ) and be able to achieve submicron spatial resolution while maintaining their stability. Both analytical and experimental results proved that CVD diamond is a good choice for the APS high heat load XBPM blade material because of its superior thermophysical properties, such as high thermal conductivity, low thermal expansion

Table 4.2 Highly Oriented Pyrolitic Graphite Filter Temperature Data Measured for Various Undulator Gaps and Stored Beam Currents (The measurements are compared with the calculations.)

\begin{tabular}{|cccc|}
\hline $\begin{array}{c}\text { Undulator Gap } \\
(\mathrm{mm})\end{array}$ & $\begin{array}{c}\text { Stored Current } \\
\mathrm{mA}\end{array}$ & $\begin{array}{c}\text { Calculated } \\
\text { Temperature in }{ }^{\circ} \mathrm{C} \\
\text { (effective conductivity } \\
\text { in W/mK) }\end{array}$ & $\begin{array}{c}\text { Temperature } \\
\text { in }{ }^{\circ} \mathrm{C}\end{array}$ \\
\hline 11.2 & 92.4 & $792(2.1)$ & 785 \\
12 & 94.1 & $776(2.0)$ & 779 \\
13 & 94.6 & $695(2.1) / 715(2.0)$ & 702 \\
14 & 95.2 & $605(2.25)$ & 605 \\
15 & 95.7 & $550(2.1)$ & 545 \\
16 & 96.3 & $463(2.25)$ & 468 \\
17 & 96.8 & $400(2.25)$ & 400 \\
18 & 97.4 & $346(2.25)$ & 337 \\
20 & 97.7 & & 233 \\
\hline
\end{tabular}


coefficient, and good mechanical strength and stiffness under heating. Since 1991, an extensive metal-coated CVD-diamond-based XBPM program has been continuing at the APS (Shu et al., 1992b; Shu et al., 1994; Shu \& Kuzay, 1996; Patent Nos. 5,387,795 \& $5,404,014)$.

At the APS, each beamline FE has two photoemission-type XBPMs to monitor the $\mathrm{X}$-ray beam position and the angle (Kuzay, 1993; Shu \& Kuzay, 1994). For an undulator FE, the present XBPM head assembly design is shown schematically in Fig. 4.26a. The first XBPM has four CVD diamond blades placed vertically in pairs. The downstream XBPM has four blades also, however, here both the vertical and horizontal blades are placed singly, and the horizontal blades are slightly tilted (Fig. 4.26b). This configuration eliminates the blade shadowing problem and somewhat mitigates the contamination of the second XBPM horizontal signal from the $\mathrm{BM}$ radiation. In the $\mathrm{BM} F E s$, the XBPM is much simpler, consisting of only two vertical blades. A complete XBPM assembly comprises three parts, the mounting post, the stage assembly, and the blade head assembly. The error sum from these parts should meet the total error budget of $< \pm 1 \mu \mathrm{m}$ in position and $< \pm 0.14 \mathrm{mrad}$ in the angle of the particle beam. Both the first and second XBPM are located upstream of the user photon shutter (PS2), so they are functional whether the user shutter is open or closed (Kuzay, 1993).

The XBPM monitor has the capability to apply a bias voltage. However, the test results show that a zero bias is acceptable and has the advantage of reducing the thermal resistance caused by the bias insulator. The geometrical configuration of the APS XBPM provides a low noise environment for photoelectron current output. The XBPM was sensitive

\section{VIEW A UNDULATOR FE FIRST XBPM}

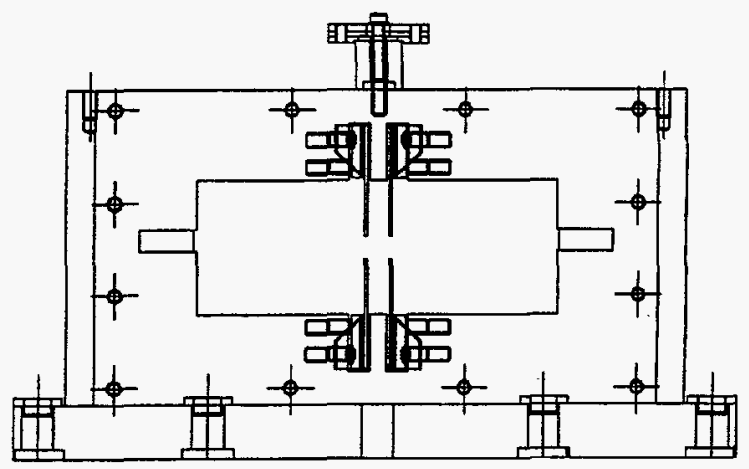

VIEW B UNDULATOR FE SECOND XBPM

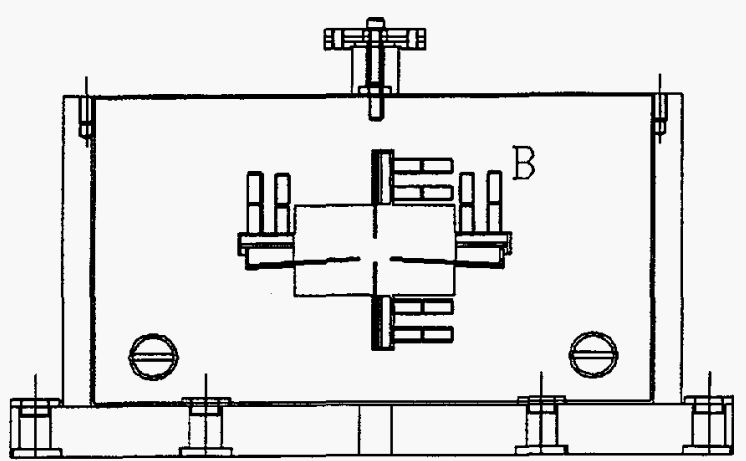

Fig. 4.26 Schematic of the XBPM head assemblies for undulator FEs, (a) first $X B P M(b)$ second $X B P M$

enough to read out the photoemission signal (about $0.6 \mathrm{nA}$ ) from a $\mathrm{BM}$ source while the APS storage ring had only a $24 \mu \mathrm{A}$ electron beam stored (during early commissioning of the storage ring in 1995). The major advantage of the XBPM is its high positioning sensitivity. Also, compared to the particlebeam position monitors in the storage ring, the FE XBPMs have much higher sensitivity to the $\mathrm{X}$-ray beam angular motion simply because they are located farther from the source

The stability of the XBPM support and the motion stages is an important consideration in the overall error budget for the XBPM. The 
XBPM main assembly is supported by a precision stage atop a mounting post. The post is a steel tube, filled with sand, and thermally insulated on the outside by ceramic cloth. This post design is very resilient to short-term temperature fluctuations (Kuzay, 1993). The XBPM stage assembly consists of stepping-motor-driven vertical, horizontal, and rotational stages. Because the commercially available vertical stages could not meet our specifications, we developed an in-house stage assembly. Test measurements using a laser Doppler displacement meter (LDDM) proved that the vertical stage attained a resolution of $<0.2 \mu \mathrm{m}$ with $1 \mu \mathrm{m}$ repeatability under a $200 \mathrm{lb}$ load (Barraza et al., 1994). Preliminary in situ vibration tests show that the XBPM main assembly maintains less than $0.1 \mu \mathrm{m}$ rms vibration displacement level with the cooling water on.

The optimized geometric design for the blades helped reduce the BM contamination. For instance, on the first XBPM on the APS 1-ID $\mathrm{FE}$, the BM contamination has been determined to be about $10 \%$ of the signal from the $2.4 \mathrm{~m}$ undulator $\mathrm{A}$ with a $15.8 \mathrm{~mm}$ magnet gap. Work at other synchrotron radiation sources has shown that contamination signals caused by the BM-emitted radiation is a major problem (Mulhaupt, 1995; Warwick et al., 1995). Problems are exacerbated for the XBPM when the undulators operate with different gaps, because the percentage level of the contamination will be variable. The regular XBPM calibration process can only provide signal correction for one set of conditions. During normal operations, the IDs function at varying storage ring currents, particle orbits, and undulator gaps. To offset the XBPM sensitivity to such operational variables, a newer XBPM system has been designed and a prototype built and tested for the APS.

\section{Smart Photon Beam Position Monitor}

This new XBPM system has an intelligent signal processor, which provides a selfcalibration function to serve as a noise and contamination signal rejecter to improve the system sensitivity and reliability (Shu \& Kuzay, 1996).

The new XBPM system configuration includes:

- a pair of photoelectron emission-style beam position monitors using CVD diamond blades for undulator beamline FEs

- a set of photoelectron current preamplifiers

- a preamplifier auto-ranging controller and digitizer

- a digital signal processor (DSP) with EEPROM database and ID source setup input interface for normalization

- a system controller with motor driver and encoder interface for XBPM calibration processes

This new system, the so-called smart photon beam position monitor (SBPM), has a built in EEPROM memory that is large enough to "remember" a complete calibration database covering all of the possible operating conditions. During the calibration mode, the monitor system controller initializes a series of automatic scan motions for the XBPM with 
different ID setup information and records them into the EEPROM database array. With the XBPM operating, the system corrects the normalized output according to the undulator setup information and the calibration database. So that, with this novel system, the XBPM is always calibrated.

The heart of the smart system is a DSP TMS320c40 from Texas Instruments, Inc., which is a floating-point processor designed specifically for digital parallel processing and real-time embedded applications.

As a part of the SBPM system, a high performance preamplifier with an intelligent controller and digitizer has been designed and manufactured.

The analog part of the system is a modular, multichannel current preamplifier developed with ITHACO. The 19" rack chassis accommodates eight preamplifier channels; the ninth slot is for the APS-designed Z-180 microprocessor-based controller/digitizer. The preamplifier with controller has selfcalibration and auto-ranging capabilities. The 24-bit parallel digital output includes a 16-bit A/D signal with a $10 \mu$ s conversion time and 8-bit data transform information, such as amplifier gain selection, channel word and end of conversion. The preamplifier system provides $10^{4} \mathrm{~V} / \mathrm{A}$ to $10^{10} \mathrm{~V} / \mathrm{A}$ auto-ranging gain control in decade steps and a DC $300 \mathrm{~Hz}$ bandwidth.

Recent additions to the above electronics are a DSP and a system controller interface (SCI) to include an EEPROM chip, a central processing unit (CPU), and D/A converter. The SCI receives the data on the ring current, ID magnet gap, and the ID source characteristics. The normalized data from the XBPM are written on the EEPROM in a series of rapidly scanned calibration runs. (See Fig. 4.27 for a schematic of the smart XBPM electronics.) Therefore a good data bank is created in the EEPROM for the correct beam center at varying gap, current, and undulator characteristics. This allows the XBPM to have memory for changing conditions. Currently we have an SBPM installed on SRI-CAT Sectors 1,2 , and 3. However only the one on the 1-ID FE has been thoroughly calibrated to date. The other two are expected to be fully calibrated by this summer.

The most recent data for beam position and beam angle from the ID FE XBPMs with and without the DSP correction are summarized in Table 4.3. All data were taken on the 1-ID FE as the undulator A gap was varied from $11 \mathrm{~mm}$ to $30 \mathrm{~mm}$.

An examination of the data in the table shows that the DSP correction has improved very significantly the apparent $\mathrm{x}$-ray position measured by the XBPMs as the undulator gap is varied from $11 \mathrm{~mm}$ to $30 \mathrm{~mm}$. As for the beam angle, the vertical angle indication of 280 microradians was reduced to a few microradians with the DSP corrections. The horizontal angle indication of a total of 600 microradians was also reduced to nearly zero. These are dramatic improvements and prove the power and the utility of the smart XBPM.

Further improvements in this system are needed if the signals from XBPM are to be used in the feedback system of the storage ring. This work will be continued in the coming year, while DSPs will be implemented on the beamlines in operation. 


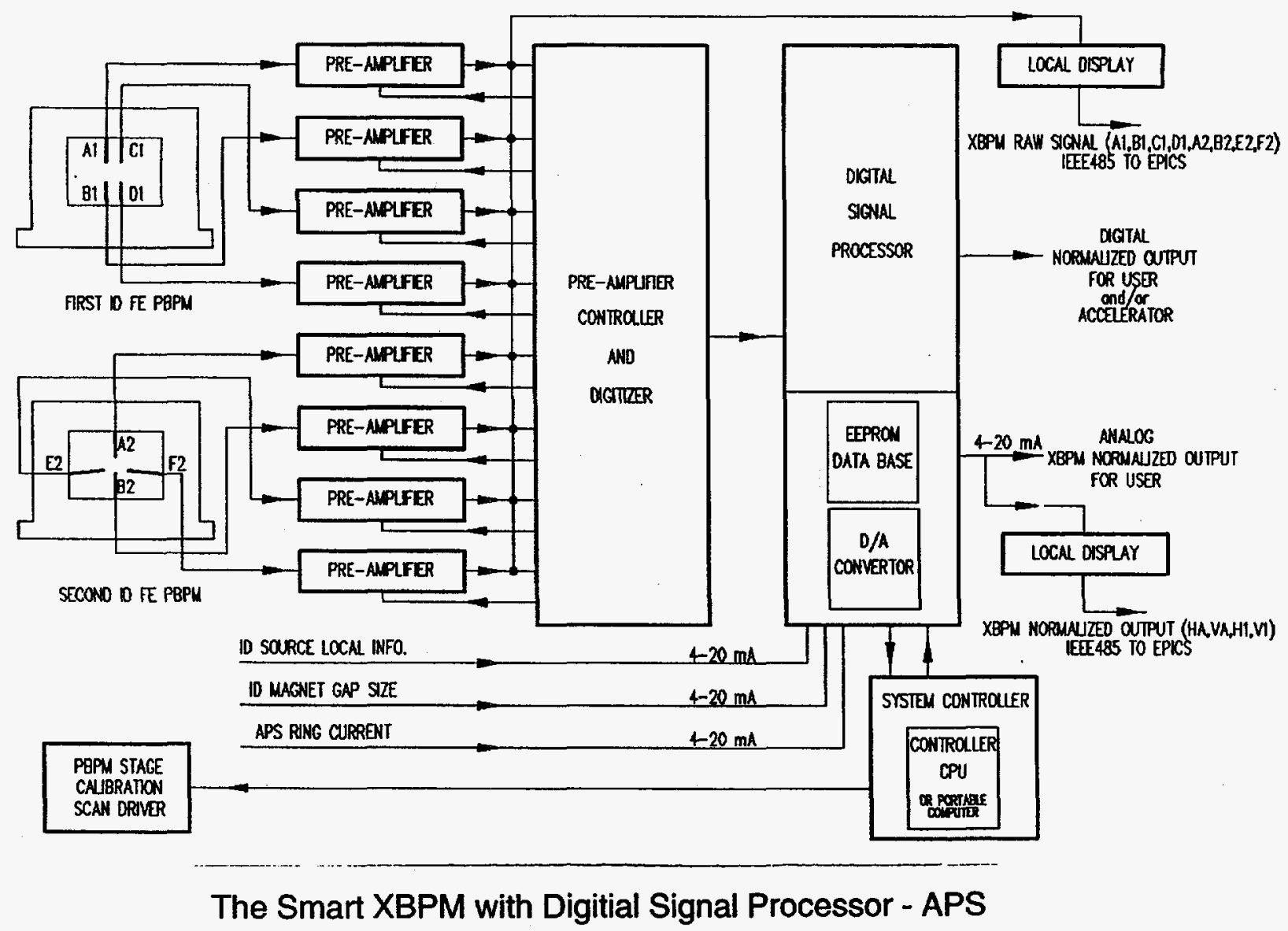

Fig. 4.27 Schematic of the electronics for the smart XBPM

Table 4.3 Improvements Obtained with DSP in XBPM Calibrations (all data taken on I-ID). Apparent $x$-ray beam position and angle measurements, with and without DSP, for varying undulator gaps from $11 \mathrm{~mm}$ to $30 \mathrm{~mm}$ (the actual positron beam being stable).

\begin{tabular}{|lrrrrr|}
\hline & \multicolumn{2}{c}{$\begin{array}{c}\text { Calibration } \Delta \text { in } \\
\text { position }\end{array}$} & & \multicolumn{2}{c|}{$\begin{array}{c}\text { Calibration } \Delta \text { in } \\
\text { angle }\end{array}$} \\
\cline { 2 - 3 } \cline { 5 - 6 } & $\begin{array}{c}\text { vertical } \\
(\mu \mathrm{m})\end{array}$ & $\begin{array}{c}\text { horizontal } \\
(\mu \mathrm{m})\end{array}$ & $\begin{array}{c}\text { vertical } \\
(\mu \mathrm{rad})\end{array}$ & $\begin{array}{c}\text { horizontal } \\
(\mu \mathrm{rad})\end{array}$ \\
\hline XPBM1 & 1300 & 100 & & \\
With DSP & 12 & 30 & & \\
XBPM2 & 325 & 2400 & & \\
With DSP & 19 & 42 & & \\
Without DSP & & & & 275 & 600 \\
With DSP & & & 6 & 8 \\
\hline
\end{tabular}

\section{Transmitting XBPM integrated with beamline windows}

It would be useful if the synchrotron beamline window could also provide the information on the position of the beam. With this in mind, a transmitting $\mathrm{x}$-ray beam position monitor was designed. The advantages of such a window will be numerous for commissioning the storage ring, FE, and beamline instruments.

In these initial studies, such a device was incorporated in the commissioning window. A CVD diamond filter, which is a $25.4-\mathrm{mm}$ diameter disk mounted on the downstream side of a fixed mask (FM3), was also designed as a transmitting $\mathrm{x}$-ray beam position 
monitor (TBPM) (Shu \& Kuzay, 1994). The monitor blade of the TBPM is mounted perpendicular to the X-ray beam, and the blade with its low- $Z$ metal coating is designed so that most of the $\mathrm{x}$-ray beam is transmitted through the blade (Warwick et al., 1995). In this design, the 160- $\mu \mathrm{m}$-thick CVD-diamond disk is coated in a pattern that forms four electronically isolated aluminum quadrants. The thickness of the aluminum coating is about $0.2 \mu \mathrm{m}$. The photoelectron emission signal is collected by a terminal interface disk, which is made from thin alumina and is coated with silver. This design concept provides the possibility of integrating the filter with TBPM functions. The beam position measurements made with a prototype of TBPM included in the commissioning window have been very instructive; the TBPM provides diagnostics capabilities during the FE commissioning, steering of particle beam orbit in the storage ring, and smart XBPM calibration. Further work will be carried out during the coming year to fully develop the system.

\section{White-Beam Slits for ID Beamlines}

Most of the ID beamlines contain a set of white-beam slits upstream of the first optical element. The major challenges in designing the white-beam slits for an ID beamline are the following:

- ability to handle high heat flux from the undulator or wiggler beam

- high precision and geometric stability requirements

- minimization of the downstream scattered $\mathrm{x}$-rays
- minimization of the slit-edge fuzziness for hard x-rays

To meet these challenging design requirements, several different types of grazing-incidence knife-edge slit assemblies have been designed and manufactured. A knife-edge slit has minimum downstream $\mathrm{x}$-ray scattering if the normal incident surface of the slit blade is facing the beam (see Fig. 4.28). Unfortunately, it is very difficult to design such a simple slit block because of the thermal load and high heat flux on the slit from the ID beam. To solve this problem, we turned the slit front surface around the $\mathrm{Y}$ axis (in Fig. 4.28) by an angle so that the $\mathrm{X}$-ray beam will impinge on the slit front surface with a grazing incident angle $\theta$ as shown in Fig. 4.29. To keep the same real recess angle $\alpha$, the grazing-incidence knife-edge block will have a back-cutting angle $\beta$ such that:

$$
\tan \alpha=\ln (1 / 1000) / \mu
$$

It is important to study the knife-edge fuzziness for a hard x-ray slit to determine an optimized real recess angle. If we define the ratio of output to input photon flux $\mathrm{I} / \mathrm{I}_{0}$ equal

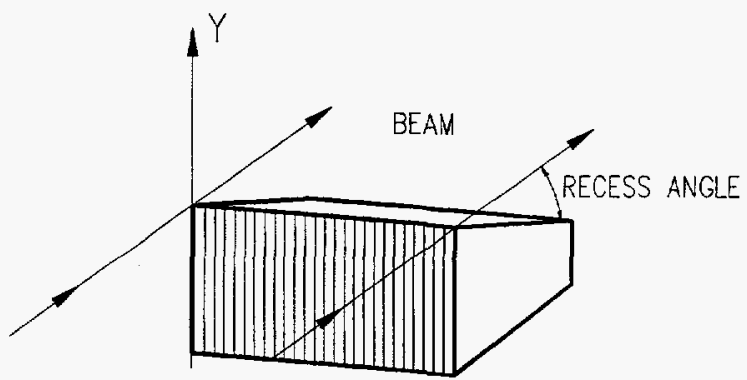

Fig. 4.28 Schematic of slit blade facing the beam 


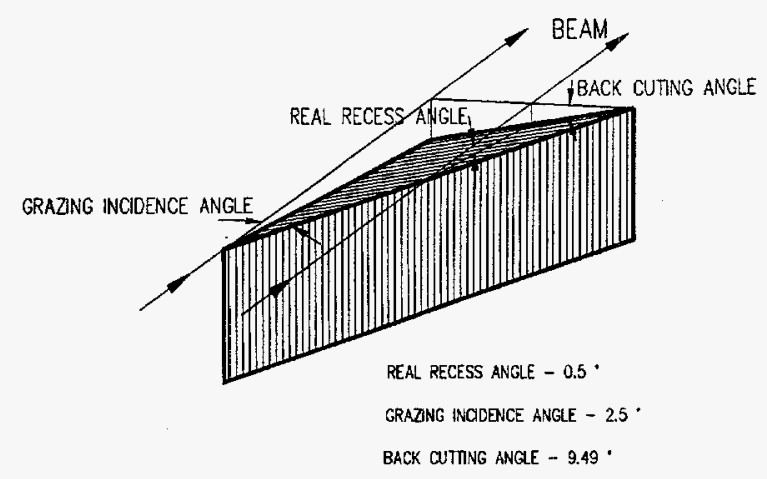

Fig. 4.29 Rotated slit blade so $x$-ray beam impinges on the slit front surface

to or less than 0.001 as the edge of the slit fuzzy area, we will have:

$$
\begin{gathered}
\mathrm{t}=\ln (1 / 1000) / \mu \\
\text { and, } \mathrm{w}=\mathrm{t} x \tan \alpha,
\end{gathered}
$$

where $\alpha$ is the real recess angle, $t$ is the depth to stop the beam (to $1 / 1000$ ), and $w$ is the knife-edge fuzziness (1/1000 definition).

Table 4.4 summarizes some characteristics of the white-beam slits designed and built for the APS user beamlines. All these slits have been installed on various SRI-CAT beamlines.
Although initial indications are such that they may have met specifications, more quantitative data will be obtained during the coming year.

\section{Integral Shutters}

An integral shutter is a device that integrates a white-beam stop, monochromatic-beam (mono-beam) shutters, a safety stop, and a collimator into one assembly to save space and to contribute to a fail-safe personnel protection system. The major function of this shutter is to deliver either the white-radiation beam or a monochromatic beam, as desired by the user, and it is usually located between the FOE (A) and a white-beam station (B). It is one of the more popular standard components and is found in most user beamlines at the APS. The integral shutters are designed to operate in white-beam mode or mono-beam mode (Chang et al., 1995), as shown schematically in Fig. 4.30. In the mono-beam mode, the double-crystal monochromator (DCM) diffracts the beam, which is then transported to a B station. If somehow the DCM fails, the defaulted white beam is stopped by the whitebeam photon shutter and the safety shutter. In the white-beam mode, the beam is transported to the $\mathrm{B}$ station.

Table 4.4 The Standard Slits for White-Beam ID Sources

\begin{tabular}{|llllc|}
\hline & Source & Max. Optical Aperture & Motion Resolution & Knife Edge \\
\hline L1-20 & Wiggler/Undulator & $48 \mathrm{~mm}(\mathrm{H}) \times 8.2 \mathrm{~mm}(\mathrm{~V})$ & $0.5 \mathrm{~mm}(\mathrm{H}) \times 0.2 \mathrm{~mm}(\mathrm{~V})$ & Yes \\
L5-20 & Undulator & $7 \mathrm{~mm}(\mathrm{H}) \times 7 \mathrm{~mm}(\mathrm{~V})$ & $0.2 \mathrm{~mm}(\mathrm{H}) \times 0.2 \mathrm{~mm}(\mathrm{~V})$ & Yes \\
L5-80 & Undulator & $4.5 \mathrm{~mm}(\mathrm{H}) \times 4.5 \mathrm{~mm}(\mathrm{~V})$ & $0.1 \mathrm{~mm}(\mathrm{H}) \times 0.1 \mathrm{~mm}(\mathrm{~V})$ & Yes \\
& & & & No \\
L5-90 & Undulator & $4.5 \mathrm{~mm}(\mathrm{H}) \times 4.5 \mathrm{~mm}(\mathrm{~V})$ & $10 \mathrm{~mm}(\mathrm{H}) \times 10 \mathrm{~mm}(\mathrm{~V})$ & No \\
\hline
\end{tabular}




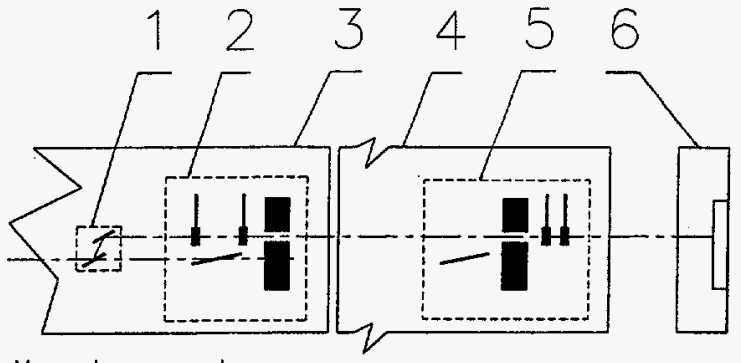

Mono-beam mode
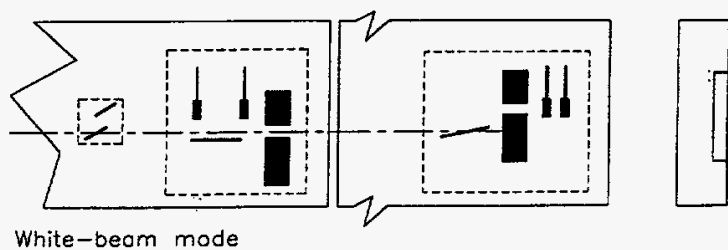

White-beom mode

Fig. 4.30 Schematic arrangement of a typical integral shutter at the APS. (1) DCM, (2) P4, (3) FOE, (4) white-beam station, (5) P5, and (6) mono-beam hutch

Integral shutters P4 and P6 have movable stops that can stop white beam during the mono-beam mode or can allow white beam to pass during white-beam mode. Users of $\mathrm{P} 4$ or P6 can use either white beam or mono beam to perform their experiments. Integral shutters P5 and P7 have fixed stops that always stop the white beam and are for users who need only mono beam to conduct their experiments. Integral shutter P8 has redundant mono-beam shutters and is typically used in a mono-beam station. Integral shutter P9 has redundant pink-beam shutters, which stop both $\mathrm{x}$-rays and bremsstrahlung. Other shutters with similar functions but different specifications are not discussed here.

With regard to safety, each mode of operation is secured by locking certain devices in their up or down positions by Kirk-key interlocks. The first mono-beam shutter and the second mono-beam shutter are interlocked to close simultaneously to provide redundant protection. In case of power failure or air failure, vacuum force and gravity force will close the mono-beam shutters so that they are fail-safe. There are four mechanical limit switches on each actuator to provide redundant signals for both the up and down positions for the safety interlock systems.

All the integral shutters mentioned above are operational and functional up to $100 \mathrm{~mA}$ operation. Of course, all the integral shutters for the BM beamlines are designed to be operated at the full power of $300 \mathrm{~mA}$. All of these units have been operating with no problems. The critical element is the photon shutter, which is constructed the same way as the FE photon shutter. Because they are usually located farther downstream in the beamline, their thermo-mechanical exposure is not as severe as that for the FE shutters.

\section{Optical Tables and Stages}

Optical support systems have been successfully implemented at the APS (Barraza et al., 1994; Barraza et al., 1995; Patent No. 5,526,903). The modular designs of the positioning stages have made it possible to quickly and effectively configure different systems, including standard optical table assemblies, mirror support and positioning systems, and other custom systems designed for experiment stations. The detailed drawings and technical specifications of all these stages are available on the DX.

The performance evaluation of these systems is undertaken using a LDDM with a measurement resolution of $10 \mathrm{~nm}$. As an example, the T4-54 vertical stage was evaluated for repeatability, accuracy, resolution, and straightness of trajectory. This was designed as part of a complex horizontally deflecting mirror system in which in-vacuum 5-degree-of-freedom 
motions are performed externally to the vacuum chamber (Barraza et al., 1995). This stage exhibits a 10 to $12 \mu \mathrm{m}$ accuracy error across the travel range.

\section{Beamline Deionized Water Production/Distribution System}

The high heat load components for the ID beamline FEs and various components of the beamlines use porous copper-mesh inserts in the flow channels to enhance the cooling performance in these units. The porous copper mesh is brazed into the cooling channels in most cases. It is vital that deionized (DI) cooling water, used to take the heat from such components, guard against biofouling, corrosion, and galvanic actions. As a policy, the FE DI water source cannot be used to supply cooling water to components of user beamlines in which compatible material selections for components cannot be strictly regulated. Consequently, the high heat load beamline components (white-beam slits, photon shutters of integral shutters, fixed masks in the commissioning window and on the differential pump) are supplied DI cooling water from separate, isolated systems, which are specified and maintained by the CAT.

The DI water production/distribution system was designed to support the needs of a user beamline based upon the following criteria:

- Deliver $70 \mathrm{gpm}$ of DI water to the sector at 140 psi pressure

- Produce high-quality DI water (3-12 Mohm/cm)

- Operate continuously with minimal maintenance
- Be compact and able to fit through the $381 / 2 " \times 451 / 2$ " access opening into the mezzanine area where the system will be installed

- Be delivered fully assembled to minimize assembly and installation time

- Be easy to use and control

The DI water production/distribution system is compact and skid mounted, and ties into a sector's supply and return main headers for distribution to the various beamlines in the sector. A separate portable skid houses the two mixed bed resin canisters that continuously polish a slip stream of the main system flow to maintain the system water quality. The polishing loop also contains an ultraviolet sterilizer unit and ultrafine filtration to keep the water free of bacteria. Water is supplied from and returned to a large 100 gallon polyethylene tank, which houses the system heat exchanger. The water level in the tank is automatically maintained via level controls. Both the technical specifications and the servicing procedures are available from the APS DX on the Web. The system is now used by most of the CATs.

\section{Bonding and Material Studies}

An important research area that has a major impact on many of the designed components is related to bonding, brazing and welding techniques to join a variety of materials including composites. The required equipment includes ovens, quick-heat-up quick-quench vacuum furnaces for general purpose brazing (see Fig. 4.31), chemical hoods for etching, cleaning and surface preparation, microscopy, 


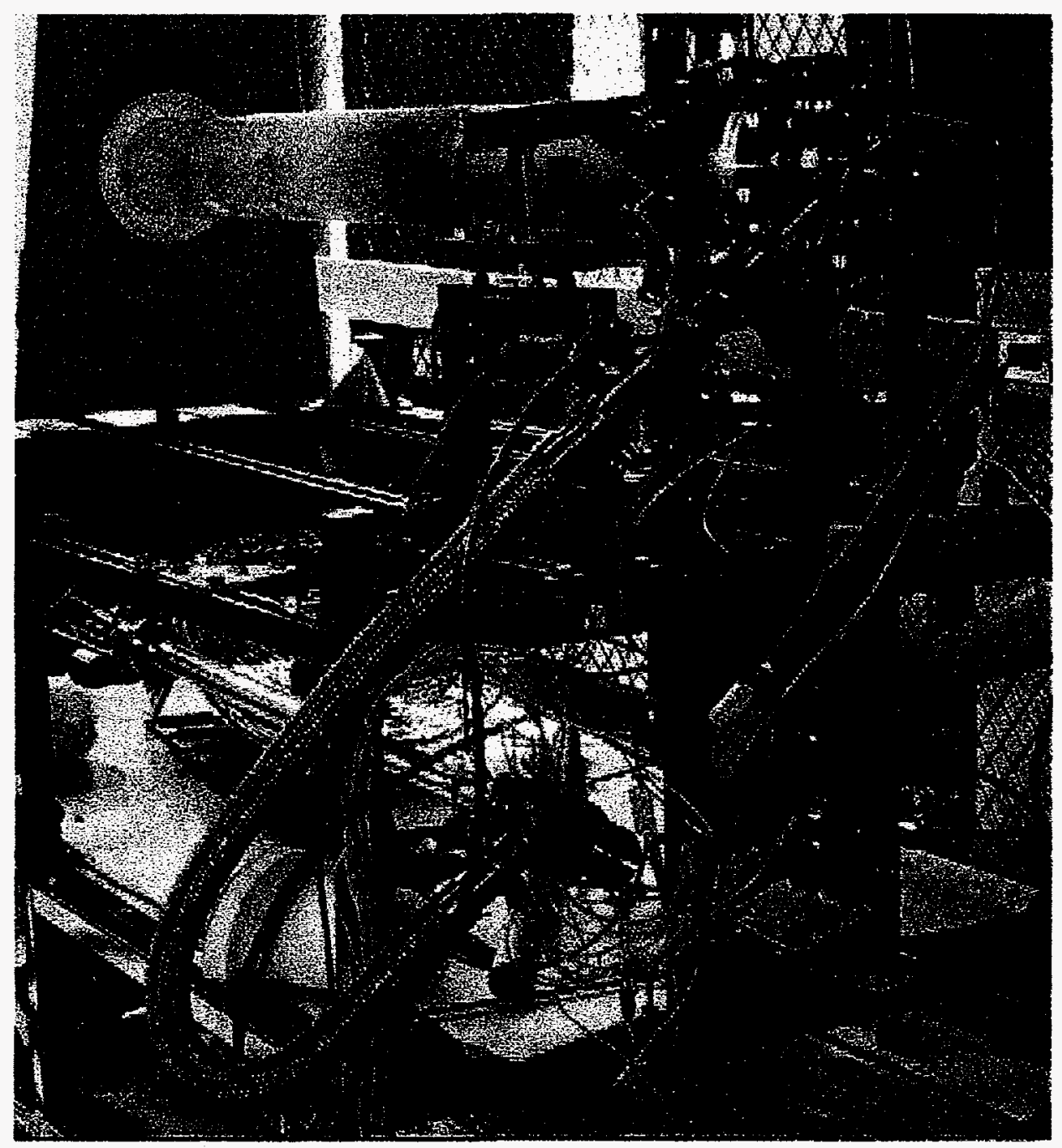

Fig. 4.31 The fast vacuum furnace heating and cooling facility is unique in the world. It is specially designed for components with high aspect ratio geometry, which is typical for high heat load components associated with large synchrotrons and third-generation light sources.

polishing and imaging facilities for metallographic and metallurgical studies. Unique expertise has been developed in the areas of Glidcop and tungsten bonding. Some of the fixed masks and photon shutters made out of Glidcop, and the tungsten slits have been brazed/bonded using these facilities. Unique capabilities developed in brazing Glidcop to Glidcop, Glidcop to OFHC copper, and Glidcop to stainless steel constitute an alternative to explosive bonding. These capabilities have benefited ASD, APS users, vendors, and other synchrotron facilities. One case in point is the fixed masks and photon shutters fabricated for SPring-8 ID beamlines.

The photon shutters and fixed masks are the components exposed to the highest heat load. The shutters are needed to stop the beam and are fabricated from high purity, high conductivity, OFHC copper with a GlidCop plate brazed or explosion bonded to the OFHC copper. The fixed masks collimate the beam and protect downstream components and beamline piping from misaligned high energy 
beams that might otherwise impinge on these surfaces (Shu et al., 1992a, 1992c). The ID fixed masks are made of GlidCop.

GlidCop is often used in applications in which the component manufacturing process uses high temperatures, such as brazing and glassto-metal sealing. GlidCop is dispersionstrengthened OFHC copper made by powder metallurgy techniques. GlidCop $\mathrm{AL}-15$ is 99.7 weight percent copper $(99.3 \%$ by volume) and 0.3 weight percent aluminum oxide ( $0.7 \%$ by volume). GlidCop annealed for 1 hour at $600^{\circ} \mathrm{C}$ has a yield strength of $400 \mathrm{MPa}$ (58 KSI). The yield strength of copper-zirconium annealed at $600^{\circ} \mathrm{C}$ is $100 \mathrm{MPa}(15 \mathrm{KSI})$, and that of OFHC copper is less than $50 \mathrm{MPa}$ (7 KSI) (Samal, 1992; Samal \& Nadkarni, 1984). GlidCop has $90 \%$ the thermal conductivity of OFHC copper, retains its strength after high temperature brazing, and has tensile strengths ranging from 380 to $700 \mathrm{MPa}$.

GlidCop has a pinned, fine-grained texture, which gives it high strength and resistance to softening at high temperatures. The pinning occurs due to the $\mathrm{Al}_{2} \mathrm{O}_{3}$ submicron particles in the GlidCop. A fast-cycle vacuum braze technology has been developed to use readily available, low-cost, silver-based alloys and to fabricate complex geometries while minimizing the diffusion. A quick braze cycle also minimizes alloying and dissolution that can degrade the quality of the joint.

Copper-to-GlidCop brazing has other complications. GlidCop components are adversely affected if the temperatures are more than $20^{\circ} \mathrm{C}$ above the melt temperature of the braze and if the melt cycle time is more than a few minutes. Expertise in brazing GlidCop with silver-based alloys has been beneficial in many ways. Stainless steel can be brazed to GlidCop by using a thermal process similar to that of brazing mesh or porous media into GlidCop. The advantage of this is that stainless-steel vacuum flanges can be brazed to GlidCop.

A photon shutter prototype has been laser tested at a power density of more than 70 watts $/ \mathrm{mm}^{2}$ for 7,000 cycles without failure of the GlidCop braze joint (Wang et al., 1994; Kuzay, 1994).

These bonding techniques developed in-house are alternatives to explosive bonding (Shu et al., 1995) in which we have also developed significant experience and which we have used extensively in making the high heat load/high heat flux standard components used for the APS FEs and beamlines.

At the APS, several tons of UHV machinable tungsten have been used for safety shutters. This UHV grade of machinable tungsten was developed by the APS working closely with two U.S. vendors.

Machinable tungsten has been successfully brazed to copper (Liu et al., 1996). Samples have been fabricated and metallurgically examined. A special fixture was developed to braze components consisting of machinable tungsten to a copper-cooling block. This capability has also been used by APS users, and the technology has been transferred to vendors.

\section{Porous Media Heat Transfer Studies Using Liquid Nitrogen Cooling}

Copper mesh, compressed and formed into porous matrices of various shapes and sizes, is routinely used in cooling channels of components for the APS FEs and beamlines to 
significantly enhance the heat transfer performance of the component. A significant amount of research has been performed at the APS over the last eight years in order to better quantify and optimize the selection of mesh porous matrix attributes (porosity, wire size, core size, bonding technique, etc.) for various water-cooled component configurations (Kuzay et al., 1991; Amir et al., 1995; Sözen \& Kuzay, 1996). The use of porous mesh matrices in cryogenically cooled components, such as mirrors and silicon double-crystal monochromators, also offers significant heat-transfer enhancement capabilities that improve the stability and resolution of the optics (Kuzay, 1992; Rogers et al., 1996a; U.S. Patent No. 5,123,982). To better understand cryo-cooled heat transfer, an experimental program has been developed to investigate the heat transfer limits using conductive porous matrices with liquid nitrogen as the coolant.

Early experimental studies show considerable promise in this heat transfer technology at liquid nitrogen temperatures. Although few studies have been done to date, excellent agreement with empirical correlation and models has been shown, and the heat transfer enhancement from the use of mesh has been demonstrated. The two-phase operating regime consisting of both liquid and vapor nitrogen is of particular interest, because the heat transfer values are enhanced to a greater degree compared to single-phase values. More studies involving non-brazed, epoxied, and brazed mesh inserts will be carried out in the future. Mesh heat transfer enhancement in cryogenically cooled components is expected to provide a high level of cooling performance for synchrotron optics.

\section{Vibration Studies}

Maintaining component stability plays an important role in beamline operations at the APS and in the success of scientific experiments. Random disturbances are prevalent on the experiment hall floor from automatic machinery used in the beamline and in the storage ring, regular noise from utilities, and pedestrian traffic. The design of beamline components requires that these disturbances are not amplified to unacceptable levels. The goal of the vibration studies is to characterize the structural dynamic response of various beamline components via experimental and theoretical techniques. In particular, the primarily concern is with the vibratory motions that occur at the micrometer level and at low frequency $(<20 \mathrm{~Hz})$.

Various experimental and theoretical studies have been undertaken during the past year that relate to the dynamic response of a standard APS optical table assembly (Barraza et al., 1994). The optical table assembly is a complex system comprising precision positioning stages and spherical bearings. The stiffness and damping properties of the flexibly suspended breadboard were estimated experimentally using modal analysis. The results show good vibration performance; the breadboard may be approximated as a rigid body when included in the theoretical analysis of the complete optical table assembly. Detailed stiffness studies for the linkages at the vertical stages were done by Basdogan and others (1996). Theoretical and experimental studies for the estimation of the damping and stiffness properties of the vertical stages are still in progress. 
Research is ongoing to improve submicron vibration measurements on systems that are of smaller mass. This issue is critical in the selection of sensors, because, in some cases, the choice of sensor may alter the inertial properties of the system under evaluation. Therefore, a noncontact approach is more suitable. Currently, a 10-nm-resolution LDDM is being used for measuring fine motions and is under development for use in low frequency vibration measurements of these smaller mass systems.

\subsubsection{Summary of Future R\&D Activities}

During the coming years, the Beamline Engineering Group will be looking into a variety of R\&D issues in support of users' needs for their beamline operations. The major ones are:

- SPring-8 FE component R\&D (see Chapter 5)

- Bonding and material studies

- DSP for the smart XBPM application

- Sub-nanoradian angular decoder development (with LDRD support)

- Cryo-cooling heat transfer

- Vibration studies on mirror tank mounts

- Mechanical design for a UHV monochromator

- Intelligent user filters that use fuzzy logic
- Sector 4 beamline design for SRI-CAT

- Position sensitive photoconductive detector (PSPCD) development (with LDRD support)

\subsection{High Heat Load X-ray Optics}

\subsubsection{Introduction}

The issue of thermal management in optical components (mirrors and single-crystal monochromators) is one of the most challenging technical problems associated with the thirdgeneration of hard $\mathrm{x}$-ray synchrotron sources. Undulators placed on the straight sections of the APS storage ring can produce a total power in excess of 5 kilowatts and power densities in excess of 160 watts $/ \mathrm{mm}^{2}$ at a typical source-to-first-optic distance of 30 meters with $100 \mathrm{~mA}$ of stored beam. It is the job of the optics designer to develop components that can operate efficiently under the influence of these high heat load x-ray beams and that can deliver the $x$-ray beam to the experiment with the least possible loss in brilliance. Among other things, this means that the thermally induced slope errors must be reduced to well below the opening angle of the $x$-ray beam itself. The enormity of the task becomes quite evident when one considers that the radiation emitted from undulators at the APS has a vertical opening angle of only 15-20 microradians or about 3-4 arc seconds full width half maximum (FWHM).

At first, the problem may seem simply one of removing heat absorbed in the optics; however, removal of the deposited power is only one ingredient in the larger challenge of 
controlling thermal distortions in precision optical components. When developing a thermal management plan for an optical component, one must consider not only the method of cooling (water, liquid metal, or cryogenic liquids flowing through slots, holes, or porous media, for example), but also the materials properties of the component (thermal conductivity, expansion coefficient, $\mathrm{x}$-ray absorption), power deposition (quantity and apportionment), heat flow (magnitude and direction), and the resulting temperature distributions that will give rise to distortions of the component.

It was recognized early in the planning stages for third-generation hard $\mathrm{x}$-ray synchrotrons that the power emitted by IDs, undulators in particular, might limit the quality of the monochromatic beam. In response, an impressive world-wide effort was mounted by researchers at many synchrotron facilities to find a solution. It is a credit to these laboratories that much of the research was complementary, with each devoting their effort to separate aspects and approaches contributing their own unique expertise to the problem. Here at the APS, impressive progress has been achieved with two approaches for high heat load crystal monochromators: cryogenically cooled silicon and room temperature diamond. A double-crystal monochromator, whose design was specified well before the optimal cooling approach had been finalized, has been successfully used for testing the various approaches. In addition to the progress made in crystal monochromators, a side-cooled mirror based on a design concept developed here has been installed and tested on one of the SRI-CAT ID beamlines and found to perform very well with little, if any, degradation in delivered beam brilliance, even under the highest power loadings.

\subsubsection{Cryogenically Cooled Silicon Monochromators}

Cryogenically cooled optics for use with high power synchrotron radiation beams were first suggested in 1985 for mirrors (Rehn, 1985) and in the following year for crystal monochromators (Bilderback, 1986). As was pointed out in those papers, the advantage of operating single-crystal-silicon optical components at cryogenic temperatures is twofold: (1) the thermal conductivity, $\mathrm{k}$, increases by nearly a decade in going from room temperature to liquid-nitrogen temperatures, while (2) the coefficient of thermal expansion, $\alpha$, decreases from its room-temperature value of $2.6 \times 10^{-6} / \mathrm{K}$, going through zero at $125 \mathrm{~K}$, and then remains slightly negative near the boiling temperature of liquid nitrogen. Consequently, the thermal gradients and resulting strain are much lower in cryogenic silicon monochromators compared to room temperature silicon for a given set of conditions. (Any thermo-mechanical strain introduced into the crystal impacts the quality of the monochromatic beam.) A significant contribution that the APS has made to this field is the use of internally cooled optics, that is, optics with liquid nitrogen flowing through the component rather than in a contact-cooling arrangement in which the liquid nitrogen flows through a heat sink in good thermal contact with the optical component (Knapp et al., 1994, 1995). The internal-cooling approach provides more effective removal of power deposited in the optic than does the contact-cooling approach, thereby keeping the optic at an lower overall temperature. The primary technical difficulty to overcome when using this approach was the development of a vacuum-tight seal between the coolant manifold and the optical component that is radiation hard, withstands thermal cycling, and introduces minimal strain into the crystal. 
This problem is exacerbated by the fact that the desired thickness of the diffracting crystal is typically less than one millimeter.

Our goal has been to develop cryogenically cooled silicon monochromator systems that will deliver near theoretical performance over the widest possible functional range of IDs at the APS. Because there is no standard monochromator design at the APS and there is an extended operational envelope, all of the monochromator, crystal, and cooling system designs were developed to have the widest possible range of applicability without major modifications, with the following general characteristics: high radiation resistance, high vacuum compatibility, ability to withstand thermal cycling, and no significant stress or vibration transmitted from the cooling manifold and piping to the optic. This comprehensive approach has worked. Of the 21 sectors currently under development at the APS, 17 have so far opted to use cryogenically cooled silicon monochromators on their ID beamlines. Several of these beamlines are currently operating routinely with liquid-nitrogen-cooled monochromators, providing high quality monochromatic beam for use in scientific experiments.

Almost all of the ID beamlines at the APS use an undulator as the primary source. Consequently, most of the development effort has been directed at designing optics for the undulator beamlines. Undulator radiation is an ideal candidate for the application of cryogenically cooled silicon crystals. By limiting the radiation striking the cooled optics to just the central cone of the undulator, the power incident on the crystal can be reduced from 5000 watts to about 700 watts, an important consideration because the capacity to remove large quantities of heat with liquid nitrogen is limited. The liquid nitrogen boil-off rate will also significantly decrease as the absorbed power is reduced. Absorbed power can be further reduced by designing the portion of the crystal where the beam strikes to be thin (in general $<1 \mathrm{~mm}$ thick). The focus of our attention has been on the optimization of thin cryogenically cooled crystals for undulators; however, investigations have been made to measure the performance of thick silicon crystals on the APS undulator (see below) and at CHESS on a high power wiggler beamline.

The advantages of thin crystals are:

- Less absorbed power (and therefore less liquid nitrogen consumed)

- Smaller thermal gradients perpendicular to the diffraction surface

The disadvantages are:

- Heat must flow through a thin membrane rather than a full 3-D solid

- Fabrication is more difficult

- Crystals are more susceptible to mechanical strain

The advantages of thick crystals are:

- Easier to fabricate

- Crystals are less susceptible to mechanical distortions

- Cooling geometry is not as limited as for the thin case 
The disadvantages are:

- Most of the X-ray power is absorbed (more liquid nitrogen consumed)

- Crystal heat exchanger must be larger

- Larger thermal gradients normal to the surface

One of the crystal designs that has been developed at the APS is shown with its coolant distribution manifold in Fig. 4.32. The thin element of the crystal is fabricated in a monolithic block of (111)-oriented silicon by milling slots in the top and bottom faces, leaving a region approximately one-half $\mathrm{mm}$ thick. A third slot is milled in the downstream face through which the transmitted beam passes. A maximum horizontal beam size of $2.5 \mathrm{~mm}$ can be accommodated, which includes essentially the entire central-cone radiation from the undulator. The downstream face of the crystal is visible, showing the slot through which the transmitted $\mathrm{X}$-ray beam passes. Also shown is the array of coolant channels on either side of the diffraction element. The seal between the Invar manifold and the silicon is made via In-coated metal C-rings. Sealing pressure is maintained by using Belleville ${ }^{\circledR}$ spring washers on the clamping screws. The mounted crystal assembly is supported on a kinematic plate

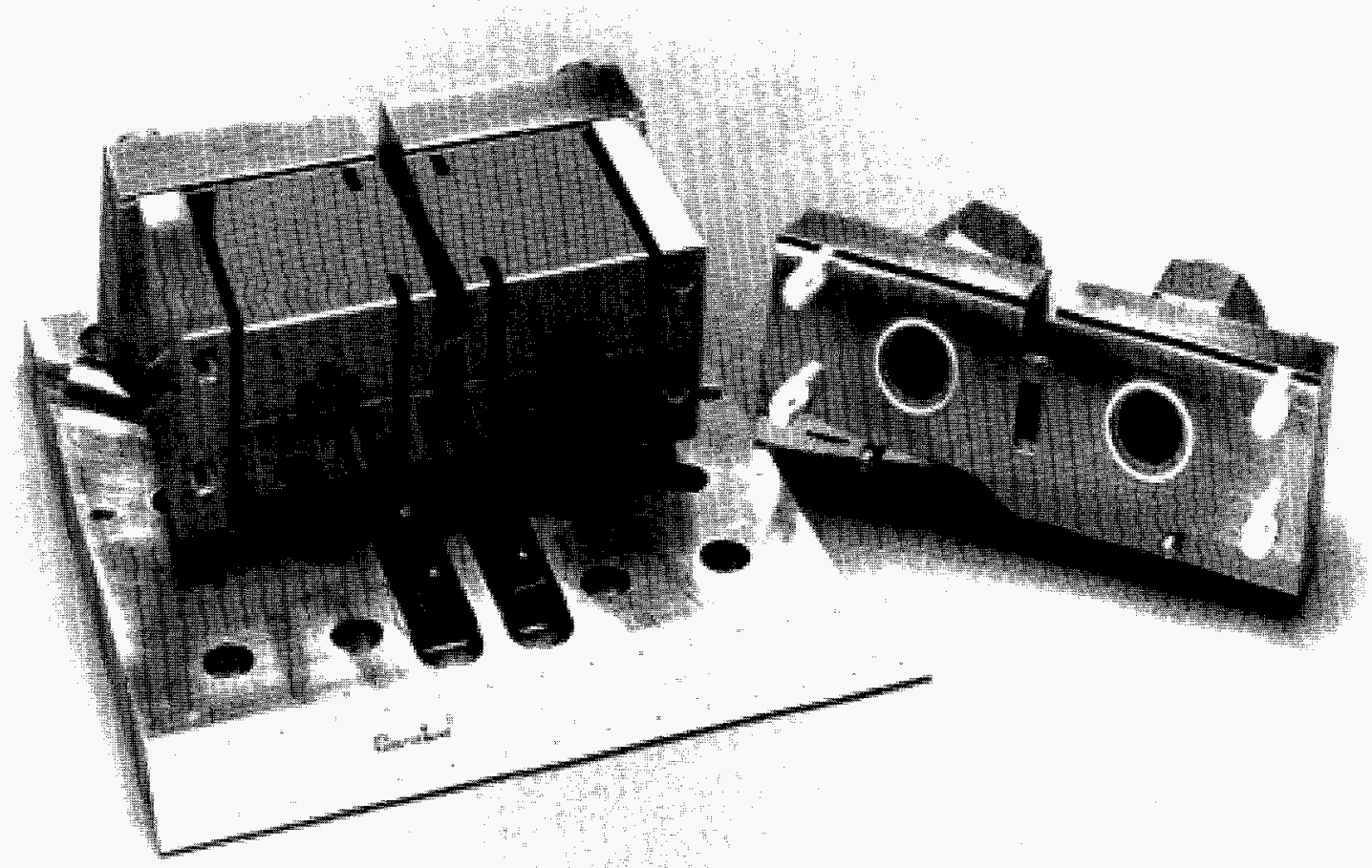

Fig. 4.32 Photograph of the cryogenic, thin silicon crystal and its coolant manifold, which have been tested on an undulator beamline at the APS. The $x$-ray beam impinges on the bottom of the slot cut into the top surface of the crystal. Visible is the downstream face, showing the slot that allows the transmitted beam to pass through the crystal, and the coolant channels on either side of the diffraction element. 
that allows for unconstrained thermal expansion while preserving the absolute position of the thin diffraction element relative to the $\mathrm{x}$-ray beam.

In the summer of 1995, this crystal was first tested on an APS undulator beamline (Rogers et al., 1996b). The beam passed through a temporary commissioning window consisting of $0.50 \mathrm{~mm}$ of graphite, $0.17 \mathrm{~mm}$ of CVD diamond, and $0.50 \mathrm{~mm}$ of $\mathrm{Be}$ and slit to limit the beam on the crystal to $2.0 \mathrm{~mm}$ horizontal $\times$ $2.5 \mathrm{~V} \mathrm{~mm}$ vertical. (About $12 \%$ of the total beam power was absorbed in the window assembly at an undulator gap of $11.1 \mathrm{~mm}$.) A broadening of the rocking curve widths is the signature of thermal distortions. Because the thermal broadenings are expected to be small, on the order of arc seconds, a higher-order reflection is monitored, in this case the (333). Rocking curves as a function of photon energy are shown in Fig. 4.33 for a fixed undulator gap of $11.1 \mathrm{~mm}$ corresponding to a deflection parameter, $\mathrm{K}$, of 2.57 . This situation simulates far worse heat loads than would normally be encountered because the undulator gap was kept at $11.1 \mathrm{~mm}$, corresponding to a first harmonic energy of $3.27 \mathrm{keV}$, for all of the rocking curves and was not opened to track the harmonic as the diffracted photon energy was increased, which would normally be the case. As the gap is opened, the power rapidly decreases. For example, for a first harmonic energy of $8 \mathrm{keV}$, corresponding to a gap of about $18.3 \mathrm{~mm}$, the incident power and peak power density are only about 40 percent of that at a gap of $11.1 \mathrm{~mm}$. Consequently, for typical operation in which the gap (i.e., harmonic) is matched to the diffracted photon energy, the monochromator should perform equally well at much higher currents. The nearly constant width of the (333) reflection as a function of energy is due not to thermal strain but rather to fabrication/mounting strain.

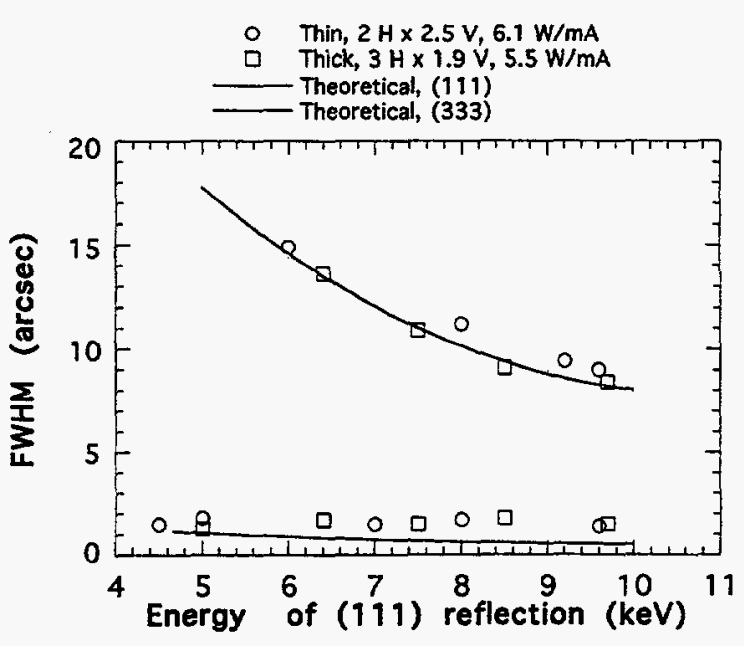

Fig. 4.33 First- and third-order rocking curve widths (FWHM) as a function of photon energy at a fixed undulator gap of $11.1 \mathrm{~mm}$. The energy of the $x$-rays diffracted by the (333) planes is three times the abscissa value. The beam incident on the thin crystal measured $2.0 \mathrm{H} \times 2.5 \mathrm{~V} \mathrm{~mm}^{2}$ with a power of $6.1 \mathrm{~W} / \mathrm{mA}$, and the beam on the thick part of the crystal measured $3.0 \mathrm{H} \times 1.9 \mathrm{~V} \mathrm{~mm}^{2}$ with a power of $5.5 \mathrm{~W} / \mathrm{mA}$. The storage-ring current ranged from 61 to $96 \mathrm{~mA}$ for the thincrystal data and from 89 to $95 \mathrm{~mA}$ for the thick-crystal data.

The performance when the beam was allowed to strike a thick portion of the crystal is also displayed in Fig. 4.33. The thick crystal data were taken from the top surface of the monochromator crystal laterally adjacent to the diffraction slot of the thin element. Obviously, the cooling geometry for the thick crystal data is not optimum because the heat flows predominantly to only one set of coolant channels; the other set is thermally isolated by the diffraction slot. Nonetheless, the thick crystal performed much better than our expectations and actually exhibited narrower rocking curves than the thin part of the crystal. This is due primarily to the lower mechanical strain in the thicker portion of the crystal. 
Similarly designed, cryogenically cooled, thin silicon crystals were tested at the European Synchrotron Radiation Facility (ESRF) using the focused wiggler radiation available on beamline BL3 (Rogers et al., 1995, 1996c). Data collected at a fixed energy as a function of incident power for the thin (0.6 to $0.7 \mathrm{~mm})$ portion of the crystals and for the thick $(>25 \mathrm{~mm})$ part are shown in Fig. 4.34. The mechanical strain in the thin parts of about 2 arcsec dominates the contribution from the thermal strain, and, therefore, the thermal component is not clearly resolvable. A slight broadening trend of the rocking curve for the thick part of the crystal is discernible as a function of absorbed power. The rocking curve for the thick section broadened to $1.7 \operatorname{arcsec}$ at the highest incident heat flux, which corresponds to a normal incidence average power density of $521 \mathrm{~W} / \mathrm{mm}^{2}$. (The numbers on the $\mathrm{x}$-axis of Fig. 4.34 take into account the spreading of the power density because of the Bragg angle of $11.4^{\circ}$.) This incident power density is in excess of what is expected for the APS undulator A in closed-

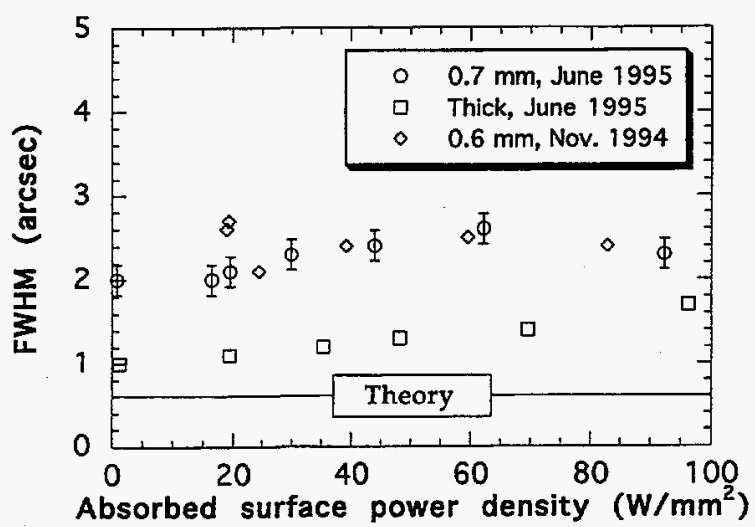

Fig. 4.34 Rocking curve widths (FWHM) for the Si (333) reflection at $30 \mathrm{keV}$ as a function of absorbed power density on the surface of the crystal. Data are shown for both thin and thick crystals. The error bars are shown only for the first data set but are the same for all. gap operation (first harmonic at $3.27 \mathrm{keV}$ ) at a storage-ring current of $300 \mathrm{~mA}$.

\subsubsection{Liquid-Nitrogen Pumps}

A liquid-nitrogen pumping system is required for each monochromator. The pumping system supplies pressurized liquid nitrogen at a variable flow rate to the optic, where it removes the heat absorbed by the optic from the $\mathrm{x}$-ray beam. The pumped liquid nitrogen then returns to the pumping system where the heat is rejected to an internal bath of atmospheric liquid nitrogen. The boiled-off liquid nitrogen is intermittently replaced from either a local or a remote storage tank or the gas is condensed in a local nitrogen reliquefier and returned to the pumping system. The pumping system is capable of handling a heat load of at least $3 \mathrm{~kW}$. It supplies a variable flow rate of up to $20 \mathrm{l} / \mathrm{min}$ at a system pressure of up to $150 \mathrm{psig}$.

The APS has expended considerable effort in developing the technical requirements for the liquid-nitrogen pumping systems to ensure that they are adequate for all of the proposed uses at the APS. The system is compact enough to be housed within the first optics enclosure next to the monochromator. The transfer hose connections are of a standard design compatible with all of the various monochromators in use at the APS. The system is wired for computer control and monitoring. Oxford Instruments has developed a commercially available pumping system based on technical specifications developed by XFD scientists and engineers, and has supplied 16 such pumping systems to APS CATs. The Oxford pumping system installed on Sector 1-ID is shown in Fig. 4.35. Several of the Oxford systems 


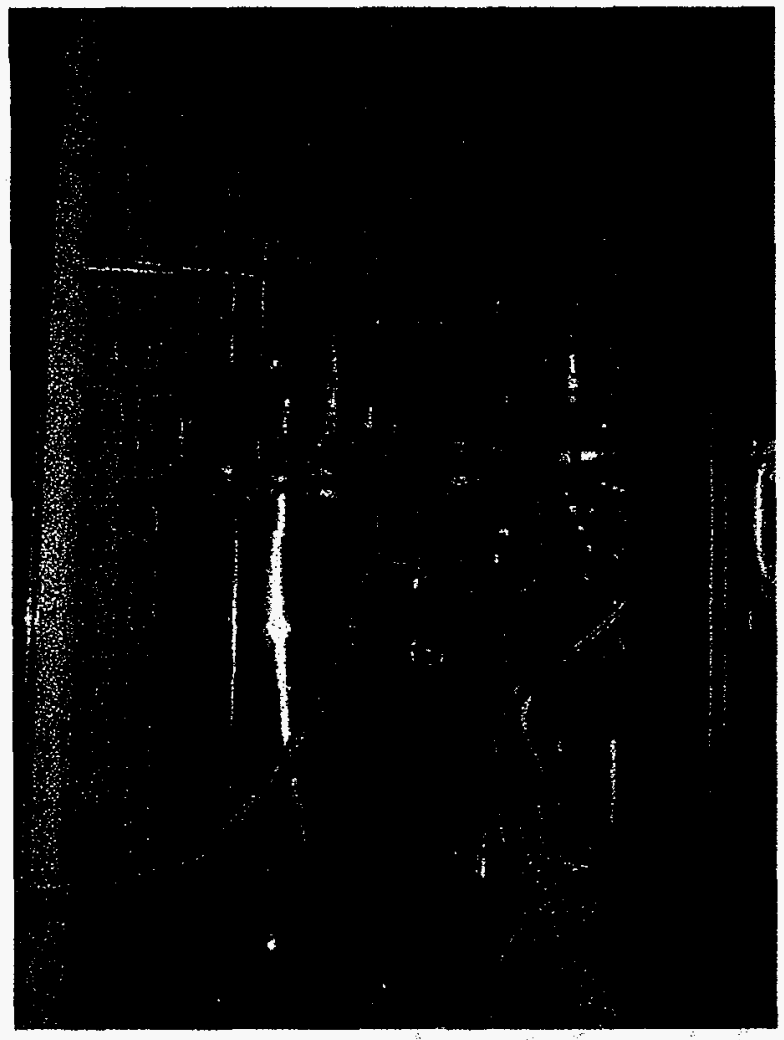

Fig. 4.35 Photograph of the liquid-nitrogen pumping system installed in the FOE of Sector 1-ID.

have been operating for almost 2 thousand hours with no pumping system failures. The uniformity of design allows for standardization of components and inter-CAT sharing of resources, providing for the greatest possible flexibility and reliability of the cooling systems at the lowest cost.

\subsubsection{Water-Cooled Diamond Monochromators}

Water-cooled diamond single crystals provide an alternative to cryogenically cooled silicon monochromator crystals. At room temperature, the thermal conductivity of diamond is about ten times larger than that of silicon, while the linear expansion coefficient of diamond is two times smaller than that of silicon. For the same absorbed power and cooling geometry, we expect that the temperature gradients and the thermal distortions in diamond will be considerably less than in silicon. Another advantage of using diamond is that, due to its lower atomic number, the absorption of $\mathrm{x}$-rays in diamond is less than in silicon: a 0.25 -mm-thick diamond crystal will absorb $17 \%$ of $8 \mathrm{keV} \mathrm{x}$ rays, while a silicon crystal of the same thickness will absorb 97\%. Thus, a thin diamond crystal will absorb a smaller fraction of the incident synchrotron beam, with considerably smaller thermal gradients and strains, compared to a similarly cooled silicon crystal (Blasdell et al., 1995).

One disadvantage of diamond monochromators is the reduction in delivered flux compared to a silicon monochromator. For energies above $6 \mathrm{keV}$, the photon flux delivered by a double-crystal diamond (111) monochromator will be about half the flux from a silicon (111) monochromator. The reduction in flux is due to the smaller Darwin width and lattice constant, which result in a narrower energy bandpass. The decrease in flux may be an acceptable trade-off when the ease of cooling a diamond crystal is considered and/or when beams with a narrow energy width are required. Another drawback of using diamond crystals is the current unavailability of perfect single crystals of appropriate size. The largest commercially available plates at this time are $7 \mathrm{~mm}$ by $5 \mathrm{~mm} .{ }^{1}$ These plates are cleaved from synthetic type $1 \mathrm{~b}$ stones and exhibit several arc seconds of mosaic spread and/or strain.

Thermal tests of the diamond were made in a double (diamond) crystal monochromator

1 Grown by DeBeers and available in the U.S. through Harris Diamond Corporation, Mount Arlington, New Jersey. 
arrangement on the Sector 1 ID beamline, using $\mathrm{x}$-ray beams produced by the $2.4-\mathrm{m}$ long undulator $\mathrm{A}$. The single-crystal plates were manufactured by Drukker International, from synthetic stones grown by De Beers, and they were supplied by Harris Diamond Corporation. Two sets of diamond plates were tested; the first set was $6 \mathrm{~mm}$ by $5 \mathrm{~mm}$ in size, with one 0.25 -mm-thick crystal and one $0.37-\mathrm{mm}$-thick crystal, and the second set was $7 \mathrm{~mm}$ by $5.5 \mathrm{~mm}$ in size with both crystals $0.44 \mathrm{~mm}$ thick. The quality of the diamonds was assessed off-line by taking $\mathrm{x}$-ray topographs at $8 \mathrm{keV}$. The data indicate that the crystals are not perfect and that the mosaic spread/strain is of the order of 5 or 6 arc seconds over the full face of the plates. While the added strain can result in a loss of brilliance in the diffracted undulator beam, it can also increase the bandpass for applications in which the flux is important, thus making up for some of the loss in throughput compared to a Si (111) monochromator.

The initial diamond thermal tests were performed with the set of smaller diamonds, with the first crystal straddling a 2-mm-wide trough on a water-cooled, nickel-plated copper mounting block (see Fig. 4.36). For the second set of tests with the slightly larger diamonds, the trough in the copper mounting block was increased to $3 \mathrm{~mm}$. In both cases, the thermal contact between the diamond and the copper was achieved by using a thin layer of $\mathrm{Ga} / \mathrm{In}$ eutectic ( $80 \%$ gallium, $20 \%$ indium); the crystals were held in place by the surface tension of the eutectic layer. The white-beam size at the first crystal position was $1.4 \mathrm{~mm}$ horizontal by $1.8 \mathrm{~mm}$ vertical for the first run, and $2 \mathrm{~mm}$ horizontal by $1.2 \mathrm{~mm}$ vertical for the second run. The first crystal diffracted $62 \%$ and $83 \%$ of the undulator central cone of radiation in the horizontal plane for the first and second runs, respectively, and over $97 \%$
TOP VEW
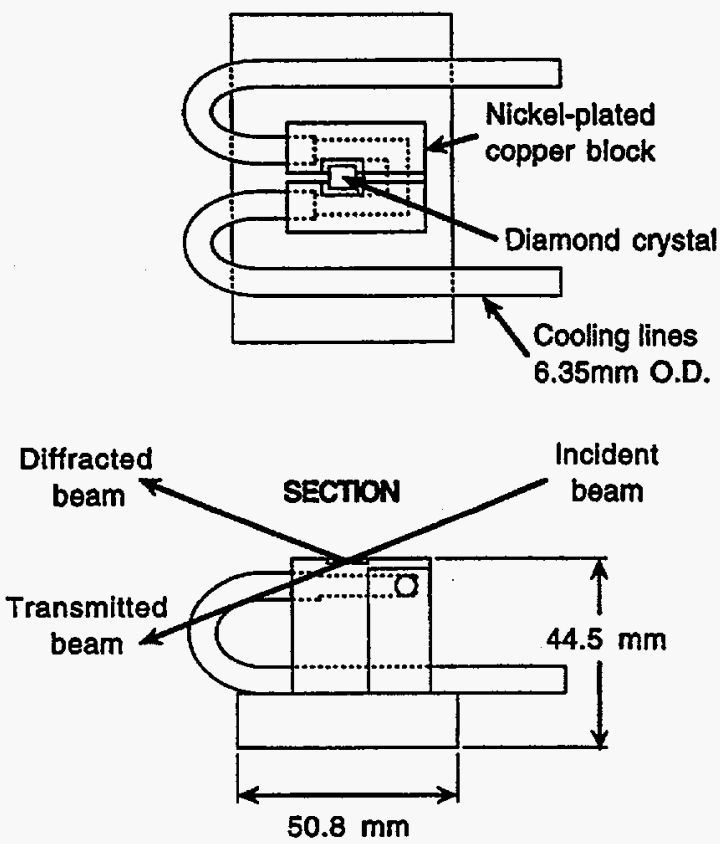

Fig. 4.36 Top view and section of the watercooled first crystal mount of our diamond double-crystal monochromator. The copper block was nickel plated to prevent the diffusion of the Ga/In eutectic into the copper. The trough in the block allows the transmission of the incident $x$-ray beam.

in the vertical plane in both cases. Rocking curves of the two diamonds were collected, and the widths of those curves were used to gauge the thermal distortion of the first diamond. As with the silicon tests, the (333) reflections were monitored for thermally induced strain because their widths are narrower than those of the first-order reflection.

Figure 4.37 shows the FWHM of the rocking curve of the diffracted beam as a function of energy for the (111) and (333) reflections taken at two energies, 6.2 and $9.7 \mathrm{keV}$, for the first set of diamonds. Also shown are the 


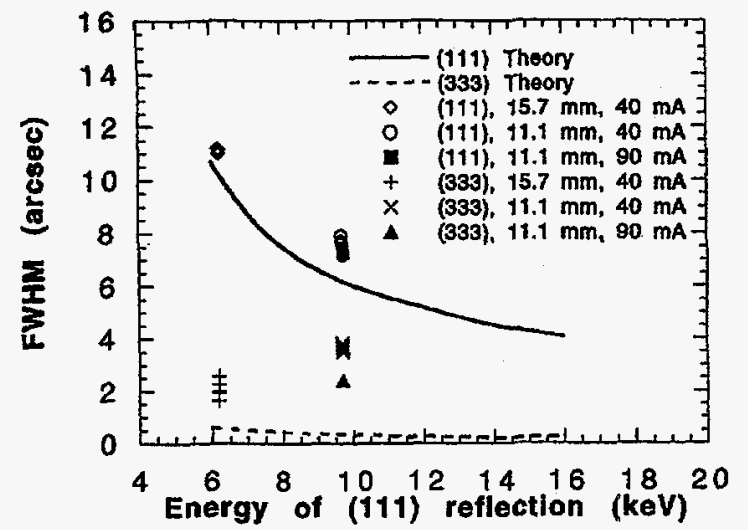

Fig. 4.37 Calculated and measured value for the FWHM of the diamond double-crystal rocking curve as a function of energy of the $x$-rays diffracted from the (111) planes. Data were taken simultaneously for the (III) and (333) reflections; the energy of the $x$-rays diffracted by the (333) planes is three times the abscissa value. The spread in the data is primarily due to diffraction for different regions of the imperfect diamond crystals and not to thermal effects.

theoretical double-crystal rocking curve widths, calculated for perfect single-crystal diamonds. The maximum power incident on the first crystal was $200 \mathrm{~W}$, with a power density of $108 \mathrm{~W} / \mathrm{mm}^{2}$ (normal incidence), at $11.1 \mathrm{~mm}$ gap and $90 \mathrm{~mA}$. Data at more energies were collected with the second (larger) set of diamonds (see Fig. 4.38). In this case, data were collected for a fixed undulator gap (11 mm) and at gaps such that the diffraction energy corresponded to either the first or third undulator harmonic. The maximum power and power density (normal incidence) incident on the first crystal were 280 watts and $123 \mathrm{~W} / \mathrm{mm}^{2}$, respectively, at $11 \mathrm{~mm}$ gap and $86 \mathrm{~mA}$. The corresponding maximum power and power density absorbed by the first diamond were 37 watts and $16 \mathrm{~W} / \mathrm{mm}^{2}$, at $17 \mathrm{keV}$. The scatter in the

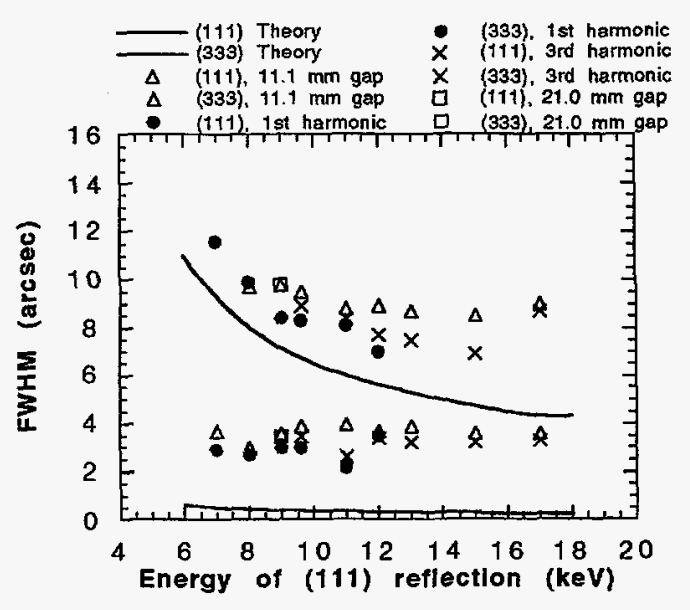

Fig. 4.38 Calculated and measured values for the FWHM of the diamond double-crystal rocking curve as a function of energy of the $x$-rays diffracted from the (111) planes. Data were taken simultaneously for the (111) and (333) reflections; the energy of the $x$-rays diffracted by the (333) planes is three times the abscissa value. The storage-ring current ranged from 77 to $86 \mathrm{~mA}$. For a given energy, the gap setting of the third harmonic data point is smaller than that of the first harmonic point; the smallest reachable undulator gap was $11 \mathrm{~mm}$.

measured FWHM of the rocking curves is attributed to small beam motions and the extreme sensitivity of the measurements to the position of the beam due to the less-thanperfect crystalline quality of the diamonds. Nonetheless, these data are encouraging from a thermal point of view, because there are no evident thermal distortions, that is, there is not an obvious increase in the width of the (333) rocking curve as a function of closing undulator gap and increasing ring current. We notice that the discrepancy between calculated and measured widths is larger for the higher energy, when the footprint of the beam on the crystals is larger and a larger portion of the imperfect diamond is being sampled. 
Also of interest is comparison of the flux through our diamond (111) monochromator and a silicon (111) double-crystal monochromator. Using the same beam size and undulator gap, we measured the maximum intensity of the (111) reflection at 6.2 and $9.7 \mathrm{keV}$ with the smaller set of diamonds and a cryogenically cooled $\mathrm{Si}$ (111) monochromator. The measured intensity ratios of the silicon monochromator to the diamond monochromator were 1.9 and 1.4 at 6.2 and $9.7 \mathrm{keV}$, respectively. The theoretical calculations for perfect crystals (Blasdell et al., 1995) predict a ratio of 1.6 at $6.2 \mathrm{keV}$ and 1.9 at $9.7 \mathrm{keV}$. The flux measurements are in reasonable agreement with the calculations and confirm the expected loss in monochromatic beam flux.

The high heat load diamond program has shown that a water-cooled diamond monochromator will perform well even under the highest heat loads on an APS undulator beamline. The drawbacks are the inherent reduction in flux compared to a silicon monochromator and the lack of perfect diamond single crystals of appropriate size. Even so, a diamond monochromator might prove a good choice for certain user applications. Working with Harris Diamond Corp., we have topographed many large uncut stones. This information is available to APS users to assist in their diamond selection. The high heat load team is also participating in an APS/ESRF/SPring-8 collaboration with Sumitomo to develop larger, more nearly perfect single crystals for use as synchrotron radiation monochromators.

\subsubsection{Cooled Mirrors}

Though most beamlines at the APS currently use a monochromator as the first optical element, there are circumstances in which a mirror is the required first optical component. Using a mirror as the first optical element can considerably reduce the thermal load on the downstream optical components (Yun et al., 1992). However, unlike crystal optics, which can be designed to transmit a large fraction of the incident radiation, a substantial portion of the incident power is typically absorbed in a mirror substrate, and so different design approaches must be applied to mirrors as compared with monochromator crystals.

One approach often adapted in high heat load substrate design is the use of very efficient cooling schemes to reduce the temperature rise, and thus the thermal gradients, in the system. Efficient cooling often implies internal cooling, in which case cooling conduits are configured in the optical substrates close to the heated surface to convect the heat away efficiently. Typical cooling schemes used are conventional channel cooling (using millimeter-size channels) (Tonnessen et al., 1996), microchannel cooling, and various enhanced cooling techniques, such as pin-post (Khounsary and Yun, 1996) or porous media cooling. Another approach to high heat load optics design, developed by XFD, is based on the recognition that efficient cooling is not synonymous with optical performance and that the key issue in thermal management is how to minimize undesirable thermal deformations and not how to minimize overall thermal deformations. In other words, the question is not how well to cool but just how to cool. In practice, this understanding leads to interesting design concepts in which location (and not efficiency) of the cooling in the optical substrate is most relevant. Because of the novelty of this design technique, referred to as the optimal contact-cooled design approach, it is briefly described here (Tonnessen et al., 1993). 
When a thermally significant $\mathrm{x}$-ray beam impinges on a mirror, the overall mirror distortion can be considered as the combination of several components, the most important of which are bending (an overall bowing of the mirror along it length), beam mapping (a distortion of the optics over the area where the power is absorbed), and a ripple distortion (due to the presence, if any, of internal cooling channels). By selecting external cooling, channel ripple distortions are eliminated. The beam mapping distortions are minimized by saturating the entire length of the optics with the incident beam to avoid sharp lengthwise heat flux falloff. The bending of the mirror is dealt with by applying a reverse thermal moment as illustrated in Fig. 4.39.

Figure 4.39a shows a long, free-standing isothermal substrate having no thermally induced deformation. If it is heated on the reflecting surface by a narrow $\mathrm{x}$-ray beam along its entire length, as illustrated in the cross-sectional view of the substrate (Fig. 4.39b), and cooled on the opposite side, it deforms into an arc. The substrate is then convex (defocusing) because of the through-the-thickness temperature gradient. Now, if the substrate is broken up into three imaginary segments, the central segment, as shown in Fig. 4.39c, is heated on the top surface, while the other two segments, as illustrated in Fig. 4.39d, are cooled on the top. When in contact, the central part tends to deform into a convex shape, while the other two segments, which are now cooler on the top than on the bottom, tend to deform into a concave shape. Thus, with proper design, a thermo-mechanically balanced substrate with no bending is obtained, as shown in Fig. $4.39 \mathrm{e}$. The cooling on the reflecting surface essentially applies a thermal moment

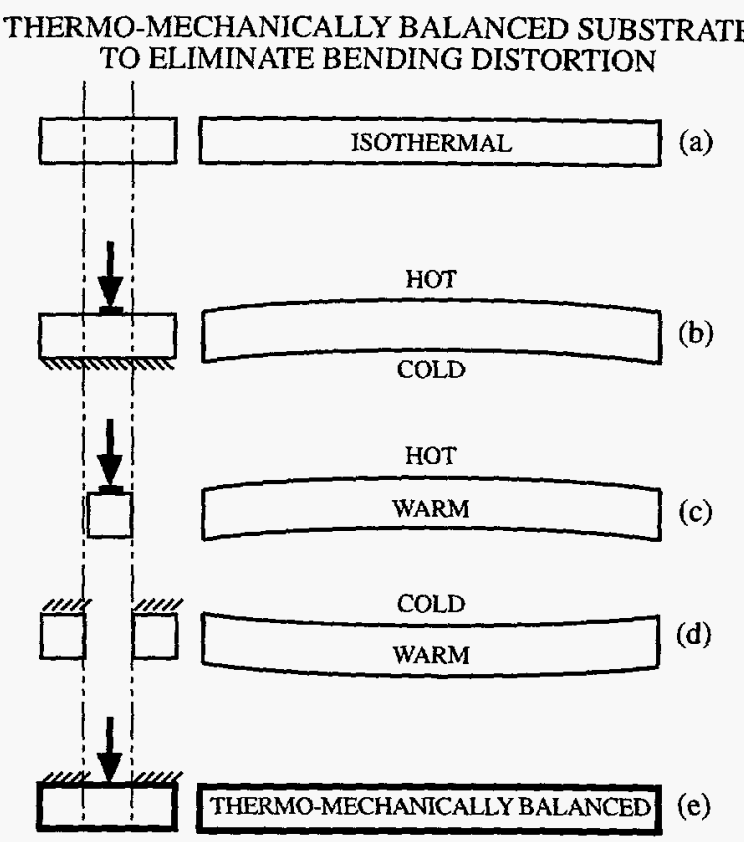

Fig. 4.39 A thermo-mechanically balanced substrate is constructed by optimal selection of the cooling sites. The substrates are shown cross sectionally on the left and longitudinally on the right above.

opposite to that produced by the central segment of the substrate. We refer to this scheme and its variations, devised to restore the desired symmetry to the system, as the reverse thermal moment technique. In actual implementation, cooling pads made of copper are placed in contact with the sides of the mirror flush with the reflecting surface to produce the same effect. This design has several advantages. They include simplicity (no internal channels, no bonding/brazing, no vacuum guards), high reliability (no radiation damage concerns, use of proven materials and techniques, reduced leak potentials), UHV compatibility, low jitter, ease of fabrication and polishing, reduced manufacturing costs, multiplicity of suppliers, and reliable and easy installation and operation. Disadvantages include a longer time constant. 
The APS had designed several mirrors based on this approach. One of them, a 1.2-m-long mirror and the first designed on the basis of the reverse thermal moment technique, is cooled by two water-cooled copper plates, $1.5 \mathrm{~cm}$ wide and $1.2 \mathrm{~m}$ long, pressed against the sides of the mirror flush with the reflecting surface. A thin layer of indium is used to enhance heat transfer from the mirror to the cooling plates. The mirror is made of singlecrystal silicon, and is $100 \mathrm{~mm}$ wide and $120 \mathrm{~mm}$ thick. A schematic of the cross section of that mirror is shown in Fig. 4.40. This mirror has been installed on the undulator A beamline at Sector 2. When the mirror intercepts the beam at 0.3 degrees, the total absorbed power and peak heat flux are about $1200 \mathrm{~W}$ and $0.5 \mathrm{~W} / \mathrm{mm}^{2}$, respectively.

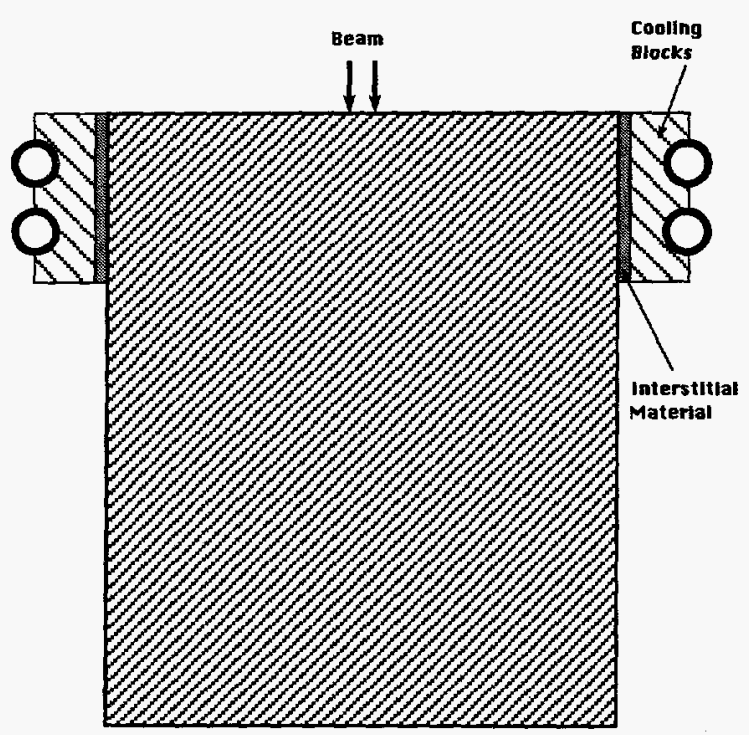

Fig. 4.40 A schematic of the cross section of the side-cooled mirror. Water-cooled copper blocks are clamped to both sides of the mirror with a thin layer of indium between the copper blocks and the single-crystal silicon mirror. The cooling blocks provide the thermal gradients to counteract the bending forces of the heat deposited by the incident beam.
Preliminary results on the optical performance of this mirror indicate a maximum steady state slope error of about $7 \mu \mathrm{rad}$ with undulator gap at $11.9 \mathrm{~mm}$ and a beam current of $78 \mathrm{~mA}$. In its final operational configuration, in which an upstream aperture is commissioned and the entire length of the mirror is illuminated with the incident beam at 0.15 degrees angle, a slope error of about $2 \mu \mathrm{rad}$ is expected. Measurements will be made to evaluate performance.

\subsubsection{Other Work Related to Cooled Optics}

When the monochromator is placed downstream of the mirror (as described above where a cooled mirror is the first optical component), the crystals will be subject to about $300 \mathrm{~W}$ total power and about $4 \mathrm{~W} / \mathrm{mm}^{2}$ peak surface power density (Yun et al., 1992). Experiments using liquid-gallium direct-cooled crystals have shown that they can handle these heat load levels. However, using the reverse thermal moment technique (as described in the "cooled mirrors" section above), we have developed a so-called "U-monochromator" that can handle these power and power density loads with contact cooling (Khounsary et al., 1996). Figure 4.41 schematically shows the U-monochromator. The two main features of the U-monochromator are as follows: (1) by careful consideration of the cooling location and use of the reverse thermal moment technique, the overall bowing along the length can be reduced, and (2) by illuminating the entire length of the crystal along the beam direction, but utilizing only the central part of the beam (as in undulator beams), sharp slope changes in the beam profile and thus in the 


\section{U-Monochromator}

A Thermo-Mechanically Balanced Design Based on the Inverse Thermal Moment Technique

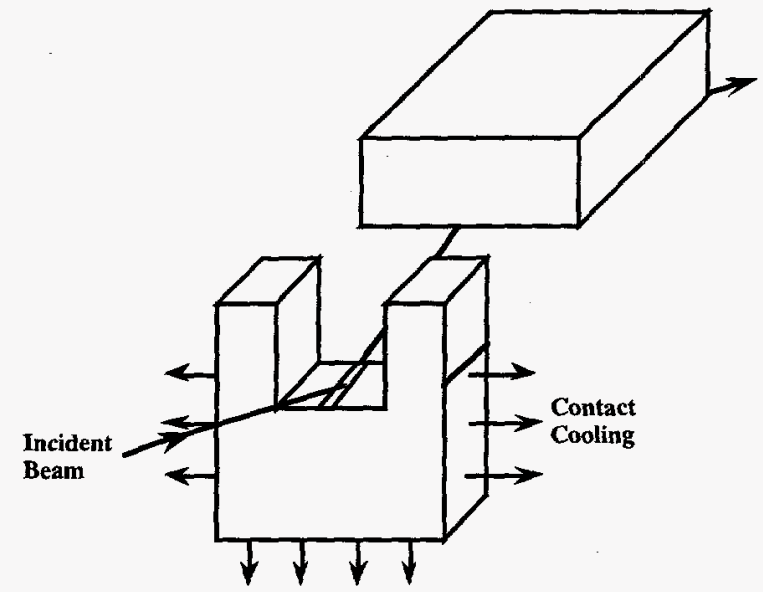

Fig. 4.41 The U-monochromator is shown schematically as the first crystal in a double-crystal arrangement. The crystal is (externally) cooled from the sides and bottom. The beam is allowed to illuminate the entire length of the crystal for optimal performance.

crystal slope errors are avoided. The latter feature is particularly suited to undulator beams because the central cone of the radiation is much smaller than the power envelope of the beam. The U-monochromator has been tested at the 1-ID beamline at the APS. The results are encouraging and show that, indeed, the measured thermally induced slope errors of the contact-cooled U-monochromator are comparable to those of direct liquid-gallium-cooled slotted crystals for the same power loading. The contact-cooled U-monochromator has the advantages of low cost; easy fabrication, mounting and manifolding; and low maintenance.

While the major effort of the high heat load group has been to study possible solutions for the undulator thermal problem, work has also been performed to provide solutions for the thermal problems on BM beamlines. Although the thermal problems of the BM beamlines are not as severe as those of the $\mathrm{ID}$ beamlines, it is still necessary to provide sufficient cooling to the crystals. The goal here is to provide a low-cost and convenient solution to the BM thermal problem. Results from computer simulations performed in 1996 suggest that indirectly water-cooled silicon crystals may perform as well as directly water-cooled ones. Tests of indirectly cooled silicon crystals are currently underway. The present scheme is to have the silicon crystals in contact with a cooled copper block. An indium-gallium eutectic layer is used to facilitate the heat transfer between the silicon and the copper block. The use of indirectly cooled crystals greatly simplifies the crystal mounting/ manifolding and the vacuum integrity issues. The simplicity of the crystals and the use of water as the coolant (in the copper block) greatly reduce the cost.

Another program currently underway is the experimental determination of thermal contact resistance across interfaces using various interstitial materials, typically gallium, gallium-based alloys, silver, or indium. This information is used in the design of contactcooled substrates, such as the contact-cooled mirrors and the diamond monochromators.

We have also looked into the use of liquid gallium as a coolant for synchrotron-radiation optics. Although currently not planned for use on undulator lines, liquid-metal-cooled optics may be important in other applications because of the ability of liquid metal to remove large quantities of heat. A dc current driven pump, based on $\mathrm{NdFeB}$ permanent magnets, has been developed by XFD and is now commercially available. The fact that this pump is driven by electromotive forces and has no moving parts minimizes maintenance and makes for easy operation. 
A key tool in the design and analysis of all the cooled optical components described above is finite element analysis (FEA). When anchored to previous experimental results, FEA allows us to accurately predict the performance of new optical designs without the costly and time-consuming process of building and testing prototypes.

\subsubsection{The Double-Crystal Monochromator Design}

The development of the technical specifications for the mechanics of a double-crystal monochromator (DCM) was undertaken by XFD early in the project. When the original specifications were written, the cooling approach for the crystals had not been finalized, and, therefore, the specifications of the monochromator had to be compatible with any of the potential approaches. The underlying philosophy behind the performance specifications of this DCM was to fabricate a device that would be useful to as many APS users as possible; that is, the design should be as generic as possible. In other words, the design should be capable of operating on both BM and ID beamlines (with appropriate changes to the cooling and crystals) with both flat and inclined crystal geometries and with a variety of coolants. The DCM should also have good scanning capabilities in the classical energy range of about 4 to $20 \mathrm{keV}$ with $\mathrm{Si}$ (111) crystals. Within the 4 to $20 \mathrm{keV}$ operational range of the DCM, windows can be used to isolate the storage-ring vacuum from the DCM and so it was specified as a high vacuum $\left(10^{-7}\right.$ to $10^{-8}$ Torr) device. The impetus for this choice was to facilitate crystal changes, to reduce the effort and time for design, and to keep the overall cost of the system at a reasonable level.
While several different DCM designs are being successfully used at first- and secondgeneration synchrotron sources, it should be noted that, because of the unique characteristics of the APS, the requirements for the APS DCM are in general more stringent. It is useful to elaborate on some of these differences. For instance, the radiation exposure levels and hardness of the radiation were expected to be significantly higher, owing to the higher energy of the particle beam $(7 \mathrm{GeV})$ than at lower energy storage rings. Discretion in the selection of the materials used inside the DCM was important. The amount of heat generated from Compton scattering off the first crystal was also a concern, and care must be exercised in the mechanical design to minimize the heating of the various components, which can cause drifts and stability problems. And finally, owing to the low particle beam emittance and long beamlines, motion resolution requirements for the APS DCMs are more stringent than those at second-generation sources. Details of the APS DCM specification have been published (Lee and Mills, 1993). Figure 4.42 shows the final motion specifications of the DCM. The contract for the DCM was awarded to Kohzu Seiki in Japan,

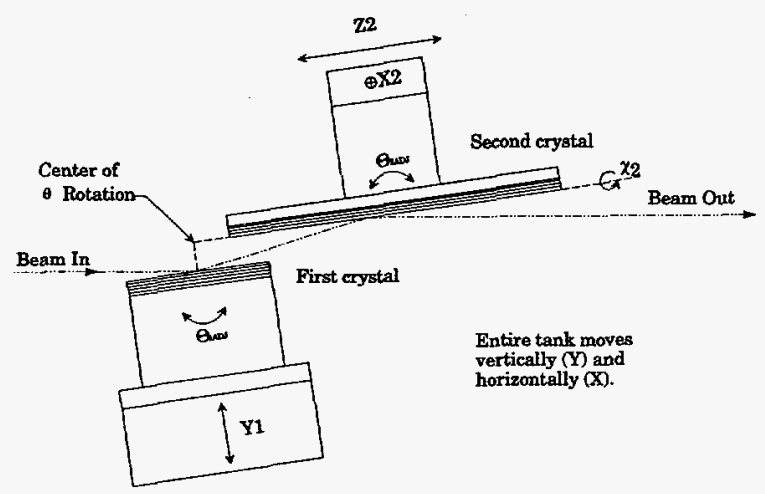

Fig. 4.42 Schematic showing all the motions of the Kohzu DCM. Almost all motions, except the overall tank motions ( $X$ and $Y$ ), are in vacuum. 
and the DCM itself, which has two symmetric crystals, was delivered to the APS in January 1994. After off-line tests and waiting for the necessary beamline construction, the DCM was installed in the beamline in 1995. The technical specifications developed for the first DCM have been used for subsequent procurements of DCMs by SRI-CAT and have served as templates/references for many of the other APS CATs.

The DCM, as delivered by Kohzu Seiki, allows for room-temperature coolants to be brought in on-axis, through the main rotation shaft. It does not, however, allow for liquid nitrogen in these feedthroughs. Liquid nitrogen is brought in (and out) through two auxiliary flanges and flexible hoses inside the DCM, between the flanges and the crystal, that allow for smooth crystal motions. Tests show that this configuration does not degrade the performance of the DCM. The DCM and the liquid-nitrogen pump have been successfully integrated into the beamline and have been performing satisfactorily since the beginning of 1996 .

In addition to the more standard DCM configuration described above, with two symmetric crystals, investigations are underway on an alternative two crystal monochromator design. For example, the so-called variable asymmetric monochromator uses two crystals with an 18 degree asymmetric cut (see Fig. 4.43). The crystals are mounted in a manner that allows one to rotate both the first and the second crystal around three separated perpendicular axes corresponding to the angles $\theta$, which controls the Bragg angle; $\phi$, which controls the asymmetric-cut angle and the incident beam angle relative to the surface of the crystal; and $\psi$, which controls the shape of the footprint of the beam on the crystal

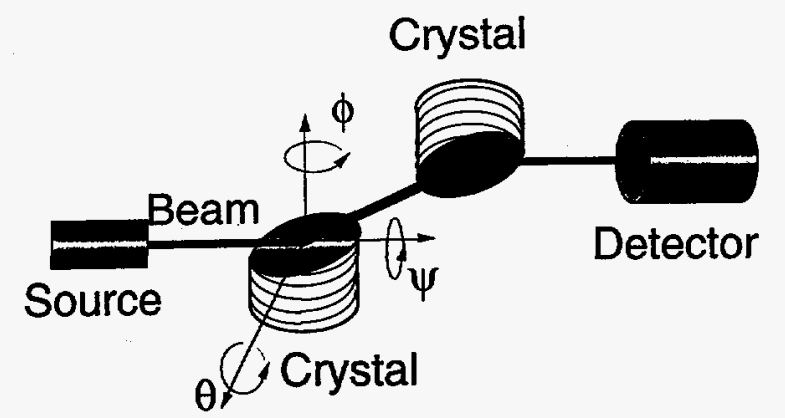

Fig. 4.43 A schematic of the variable asymmetric DCM showing the three orthogonal rotations of the first crystal.

surface. Rotation around the $\phi$ axis simultaneously increases the effective asymmetriccut angle and decreases the incident angle of the beam on the surface of the crystal with no change in the Bragg angle to first order. This allows the area of the beam footprint on the crystal to be altered, changing the thermal load per unit area, and at the same time varies the angular acceptance of the crystal for diffraction for a given incident wavelength. In practice, this allows one to increase the throughput of a narrow band of wavelengths by a factor of 10 to 20 while at the same time increasing the ratio of the area of the footprint of the beam on the crystal to the area of the incident beam by a factor of 50 . The second crystal has a similar set of rotation stages and can be moved both perpendicular and parallel to the beam direction. A detailed description of the operation of this kind of monochromator has been published (Smither and Fernandez, 1994).

\subsubsection{Brilliance/Brightness Measurements}

High heat load monochromator tests have shown that indirectly cooled diamond or direct 
cryogenically cooled silicon crystals have negligible thermal distortion under the heat load generated from APS undulator A. These results are based on double-crystal rocking curves that indicate minimal ( $<1$ arc second) thermal distortions due to the heat load. While these measurements are very useful, they do not provide direct information regarding the monochromatic beam brilliance (photons/sec/ $\mathrm{eV} / \mathrm{mrad}^{2} / \mathrm{mm}^{2}$ ) or brightness (photons $/ \mathrm{sec}$ / $\mathrm{eV} / \mathrm{mrad}^{2}$ ). Because it is the beam brilliance that exemplifies the third-generation synchrotron sources, we have embarked on a program to directly measure the monochromatic beam brilliance. This direct measurement of the beam brilliance is of particular importance to APS users who are interested in beams with very small angular and energy spread, for example, for use in inelastic scattering or nuclear resonant scattering. For such users, beam brilliance is a factor in selecting a monochromator. For a similar (hkl) reflection, the monochromatic throughput (photons/sec) of silicon is about twice that of diamond. However, the energy spread of the monochromatic beam from silicon is about twice that of diamond. For perfect crystals, therefore, theoretically, beam brilliance over a narrow energy range $(\Delta E)$ is about the same for both silicon and diamond. Because the cost and maintenance of a cryogenic system is much higher than that of a water-cooled diamond, it is important that we measure the actual beam brilliance from these two systems. The measurement scheme (see Fig. 4.44a) involves two channel-cut crystals in a dispersive $(+,-,-,+)$ geometry downstream of the DCM. Using asymmetric ( $43^{\circ}$ asymmetry) silicon (777) reflection in the channel cuts, the acceptance of such a four-bounce reflection at $14.5 \mathrm{keV}$ is about $2 \mathrm{mrad}$ and $7 \mathrm{meV}$. With such a small acceptance in angle and energy, it is possible to map out the Dumond diagram of
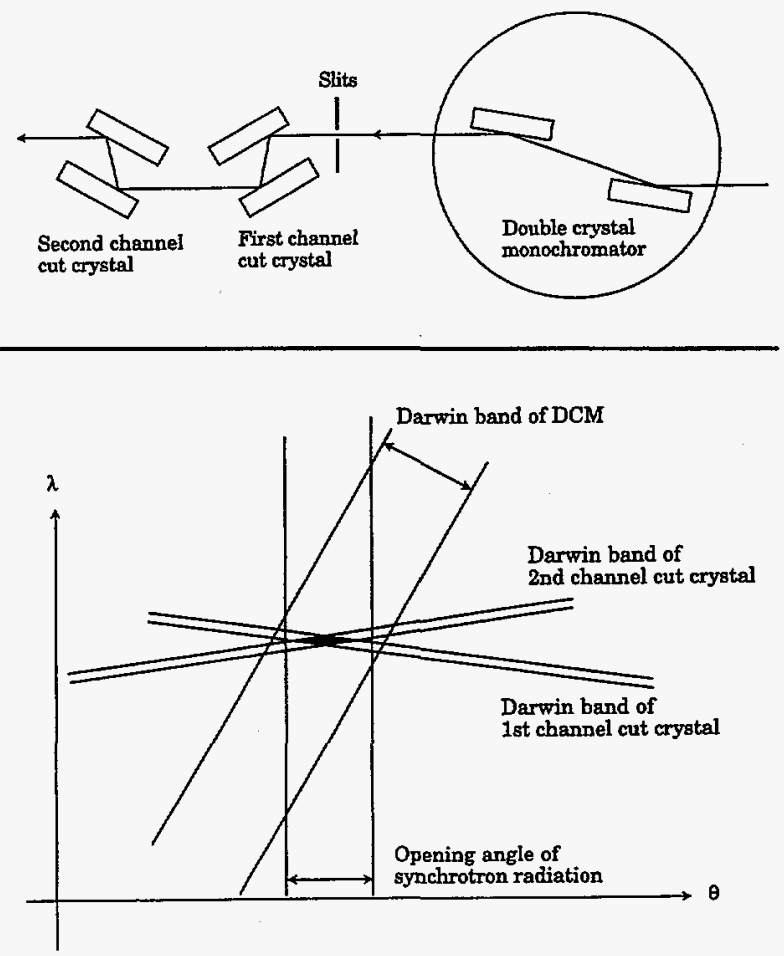

Fig. 4.44 (a) Schematic of the setup for the brilliance measurements. The channel-cut reflections used presently are asymmetrically (43 asymmetry) cut Si(777). (b) Dumond diagram representation of the setup for the brilliance measurements. The dark diamondshaped region represents the transmission of the two channel-cut crystals. By rotating the channel-cut crystals, the transmission diamond can be moved relative to the rest of the figure. If slits are not used, the beam brightness is measured. As shown, the measurements are only for the vertical direction, integrated over the horizontal direction. The diagram is not to scale.

the monochromatic beam and directly measure the beam brilliance. The Dumond diagram representation of the measurement setup is shown in Fig. 4.44b. Currently, the plan is only to measure the beam brilliance in the vertical direction integrated over the horizontal direction. 


\subsection{New Instruments and Techniques}

\subsubsection{Introduction}

The development of beamline instrumentation and techniques that demonstrate and enhance the unique opportunities that are available at the APS is undertaken primarily by XFD scientists and engineers on the SRI-CAT beamlines. Specifically, the goals of the SRICAT are:

- To develop and diagnose IDs, high heat load optics, and other novel x-ray optical components and to develop innovative techniques that will prove beneficial to the entire community of APS CATs.

- To develop and implement strategic instrumentation programs that will open up new areas of research at the APS and to extend the synchrotron radiation community beyond the current boundaries.

Some of the beamlines of SRI-CAT have been designed with flexibility in mind for the testing of beamline components and optics, while other have been designed with specific instrumentation to carry out selected areas of technique development. The later types are called strategic instruments. We currently have four strategic instruments on the Sector 2 and 3 ID beamlines:

- an instrument with milli-eV resolution

- an instrument with micro-to-nano-eV resolution to perform nuclear resonant scattering experiments
- a 1-4 keV radiation source and instrumentation for use of coherent soft $\mathrm{x}$-rays

- instrumentation for development of hard $\mathrm{x}$-ray microfocusing optics and techniques

A schematic of the locations of these programs, along with the other major programs, on the SRI-CAT beamlines is shown in Fig. 4.45. As indicated in the figure, SRI-CAT currently has developed five beamlines, three $\mathrm{ID}$ lines and two $\mathrm{BM}$ lines. The beamlines are made up of XFD-designed standard beamline components, whose designs are available to APS users. The SRICAT scientists have not only set the technical specifications for those standard components but have also commissioned them and evaluated their performance after installation

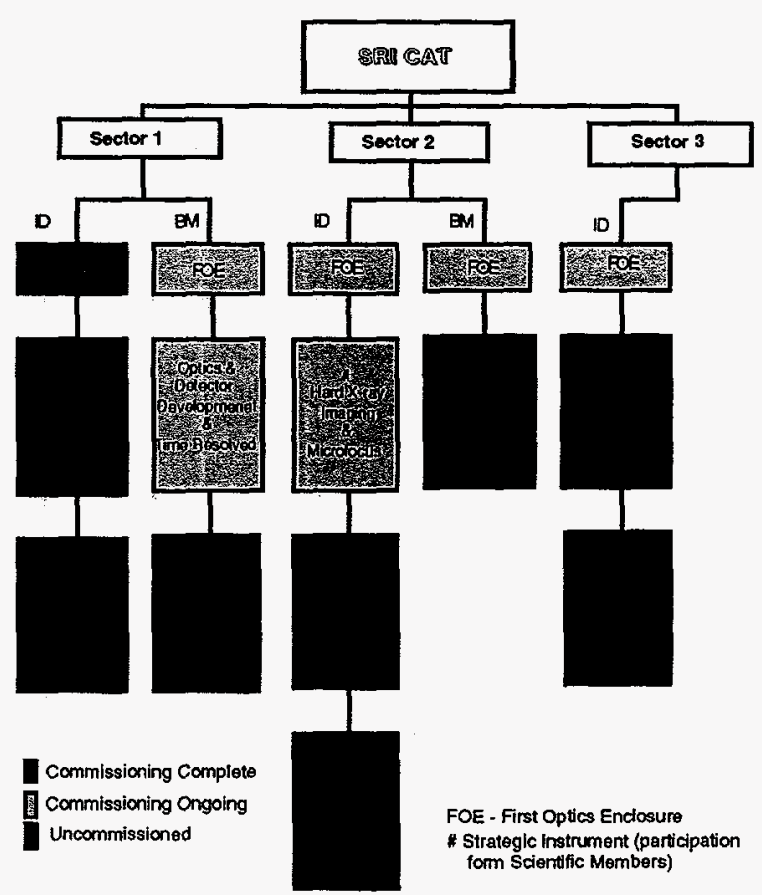

Fig. 4.45 Schematic of SRI-CAT showing activities and locations of various programs on the five beamlines. 
on the beamline. The commissioning and debugging of the standard components is a valuable service to all APS users because it should allow their beamlines to come on-line in a faster and more efficient manner. Schematics of the various beamlines and their capabilities are given below along with some of the representative science that has been performed to date.

\subsubsection{Sector 1}

Sector 1 of SRI-CAT comprises an ID beamline (1-ID) and a BM beamline (1-BM). Associated with these two beamlines are four scientific programs:

- development and characterization of high heat load optics and FE/beamline components (1-ID)

- high energy $\mathrm{x}$-ray instrumentation and experimental techniques (1-ID)

- optics and techniques for timeresolved $\mathrm{X}$-ray studies (1-BM-B)

- intation and techniques for the production and analysis of polarized radiation above $5 \mathrm{keV}(1-\mathrm{BM}-\mathrm{C})$

Although a "home" station or beamline is indicated next to each of these programs, experiments from all four programs are carried out on both beamlines to take advantage of the different characteristics of 1-ID and 1-BM. Work on high heat load optics and FE/beamline components is described elsewhere in this document.

\section{1-ID Description}

The 1-ID beamline is a flexible, general purpose ID beamline that can be used for a wide variety of experiments and is particularly well suited as a test bed for novel $\mathrm{x}$-ray optics. It has three stations (an FOE and two experiment stations). The 1-ID beamline was the first ID beamline to receive beam at the APS and was the first beamline to have all of its stations become operational.

The 5-meter-long straight section for the beamline has room for two IDs that can be alternatively operated. Currently, a standard APS undulator A is installed, with plans to add an APS wiggler $A$ in the latter part of 1997. Undulator $A$ is the most commonly used ID at the APS and produces a highly brilliant source of $\mathrm{x}$-rays that can be tuned to any energy from $3.5 \mathrm{keV}$ to over $200 \mathrm{keV}$. It is well suited for experiments and optics development in the x-ray energy range ( $6 \mathrm{keV}$ to $18 \mathrm{keV}$ ) and also proves to be a superb source for high-energy (>50 keV) experiments.

The FOE, 1-ID-A, covers the area of the beamline immediately downstream of the ratchet-wall penetration and is used to house various components that manipulate the $\mathrm{X}$-ray beam prior to transmitting the beam downstream to the experiment stations. The components in the FOE are permanent parts of the beamline and, normally, remain in place when changing to a new experiment. Among the FOE components are filters, white-beam slits, the Kohzu DCM, monochromatic-beam shutters, and a bremsstrahlung collimator/ stop. A beryllium window separates the FOE portion of the beamline from the FE (i.e., the beamline inside the ratchet wall) and, hence, the storage-ring vacuum. 
The 1-ID-B station is located directly downstream of 1-ID-A. Experimental equipment in 1-ID-B is mobile and can be swapped in and out depending upon the experiment. A photon/bremsstrahlung stop and a second monochromatic-beam shutter are located at the downstream end of 1-ID-B. This stop is the farthest point downstream that white beam can reach on the beamline. The 1-ID-C station is a large $(4 \mathrm{~m} \times 7 \mathrm{~m})$ monochromatic experiment station located at the downstream end of the 1-ID beamline. As in 1-ID-B, the experimental equipment (which includes a Huber 5020 diffractometer) can be swapped in and out depending upon the experiment.

The optical concept for the 1-ID beamline is one of the simplest at the APS. In "monobeam" mode, the white beam from the ID enters the FOE, is monochromated in the Kohzu DCM, and is then used in either 1-ID-B or 1-ID-C for experiments. In "whitebeam" mode, the white beam is passed through the Kohzu DCM into the 1-ID-B station. The beam can then be used directly in 1-ID-B, or monochromated by a temporary monochromator and passed to the 1-ID-C station. Table 4.5 summarizes the hardware on 1-ID, and Fig. 4.46 shows the beamline layout.

\section{1-BM Description}

The 1-BM beamline has a layout similar to $1-$ ID in that there are three stations in tandem (1-BM-A, 1-BM-B, and 1-BM-C), but it differs considerably in optical design. The 1-BM-A station is a FOE and houses the filters, white-beam slits, collimating mirror, DCM, monochromatic-beam shutters, and bremsstrahlung collimator/stop. A beryllium window separates the FOE portion of the beamline from the FE. The 1-BM-B is an experiment station capable of operating with white beam, "pink" beam, or monochromatic beam. The bent-crystal monochromator (BCM) is located at the upstream end of 1-BM-B and a bremsstrahlung stop is located at the downstream end of 1-BM-B. The 1-BM-C station is the end station on the beamline and accepts only monochromatic radiation. A vertically focusing mirror, monochromatic slits, and monochromatic shutter are located between 1-BM-B and 1-BM-C. The 1-BM beamline was the first BM beamline at the APS to receive beam. There are four major optical components on the 1-BM beamline:

- collimating white-beam mirror

- DCM

- $\mathrm{BCM}$

- focusing monochromatic-beam mirror

The collimating white-beam mirror is $1.2 \mathrm{~m}$ long and made from silicon. It is water cooled and coated with palladium. This vertically deflecting, cylindrical mirror has an adjustable radius, which collimates the beam and at the same time provides harmonic rejection and power filtering. It is possible to lower the mirror out of the beam to allow the white beam onto the DCM or into 1-BM-B.

The collimating mirror is followed by the DCM, which operates between $6 \mathrm{keV}$ and $25 \mathrm{keV}$ using $\mathrm{Si}$ (111) reflections. The second crystal in the DCM is a sagittally bent crystal, which provides horizontal focusing of the beam into 1-BM-C. The crystals in the DCM can easily be removed from the beam to allow white or pink beam into 1-BM-B. 
Table 4.5 Sector 1, Insertion Device Beamline (1-ID)

\section{Responsible Scientists}

Dean Haeffner, phone: 630 252-0126, fax: 630 252-0161, e-mail: haeffner@aps.anl.gov

Wah Keat Lee, phone: 630 252-7759, fax:630 252-3222, e-mail: wklee@aps.anl.gov

\section{Scientific Programs}

- Development and characterization of high-heat-load optics and front-end/beamline components

- High-energy $\mathrm{x}$-ray instrumentation and experimental techniques

\section{Source Characteristics}

- APS Undulator A (operational)

- APS Wiggler A (not yet installed)

Optical Components

\begin{tabular}{|l|c|l|l|}
\hline \multicolumn{1}{|c|}{ Component } & \multicolumn{1}{|c|}{$\begin{array}{c}\text { Distance from } \\
\text { Source (m) }\end{array}$} & \multicolumn{1}{|c|}{ Description } & \multicolumn{1}{|c|}{ Status } \\
\hline White beam slits (L5) & 28.0 & Horiz. \& vert. & Operational \\
\hline Kohzu double crystal mono. & 29.8 & LN $_{2}$ cooled Si or diamond & Operational \\
\hline Mono slits (L2) & 37.8 & Horiz. \& vert. & Operational \\
\hline
\end{tabular}

Beamline Configurations (1-BM)

- Monochromatic beam into B \& C

- White beam into $\mathrm{B}$, monochromatic beam into $\mathrm{C}$

\section{Detectors}

- NaI scintillation counters

- Ionization chambers

- Ge and Si solid state

\section{Beamline Control}

- UNIX-based Sun workstations

- VME

- EPICS

- $\mathrm{SPEC}$

Ancillary Equipment

- Huber D5020 4-circle diffractometer on motorized table

- Motorized optical tables

- $\mathrm{LN}_{2}$ pump 


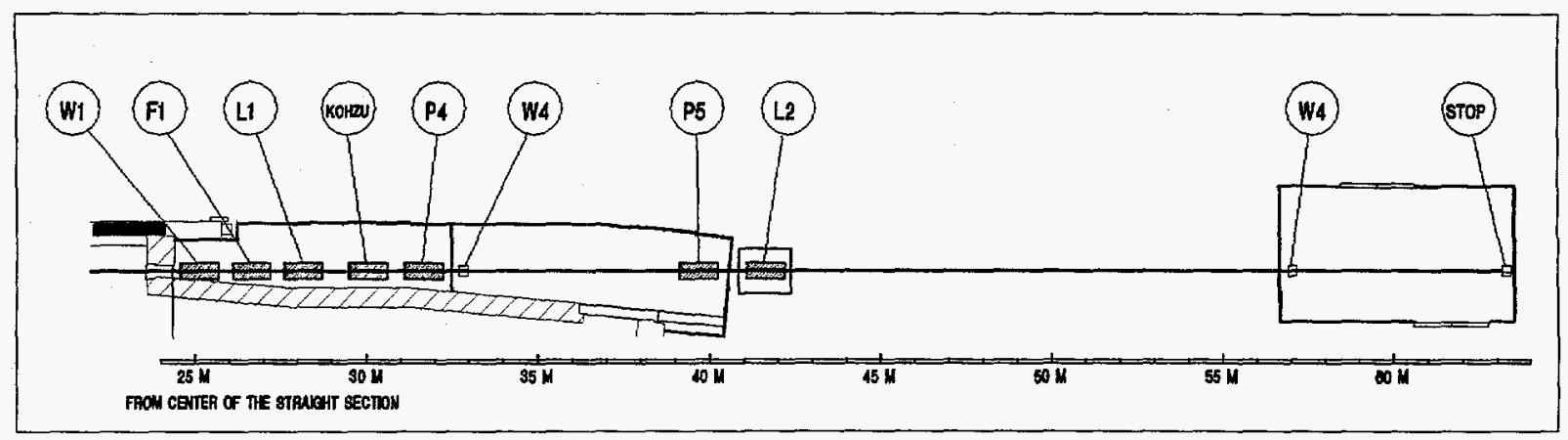

Fig. 4.46 Schematic layout of the Sector 1 ID beamline. Beam enters from the left and travels to the right. W1: beryllium window, F1: filter, L1: white-beam slits, KOHZU: double-crystal monochromator, P4: moveable white-beam stop and monochromatic shutter, W4: beryllium window, P5: fixed white-beam stop and monochromatic shutter, L2: monochromatic-beam slits, W4: beryllium window.

The BCM is located at the upstream end of 1-BM-B and can only deliver beam within this station. This monochromator uses a four-point bender with a single crystal (typically $\mathrm{Si}(220)$ ) to meridionally focus the beam. The BCM provides a polychromatic horizontal focus with a spot size of $\sim 150 \mu \mathrm{m}$ and a bandpass up to $1 \mathrm{keV}$. Its primary use is for energy-dispersive experiments. When the DCM is being used, the crystal in the BCM may be removed from the beam.

The second mirror is $1 \mathrm{~m}$ long and made from silicon. Like the first mirror, it is a palladiumcoated, vertically deflecting cylindrical mirror with an adjustable radius. This mirror provides vertical focusing of the beam from the DCM into 1-BM-C. Table 4.6 summarizes the hardware on 1-BM, and Fig. 4.47 shows a beamline layout.

\section{Current Status}

All components for undulator operation have been installed and commissioned on the 1-ID beamline. For wiggler operation, some components will require alterations and those changes are expected to be completed in calendar year 1997 . When the monochromatic mirror is installed in the fall of 1997, the 1-BM line will then be fully operational.

\section{High Energy X-ray Instrumentation and Techniques}

The goal of the high energy $\mathrm{x}$-ray program is to develop instrumentation and techniques that utilize the abundant potential of the APS as a source of photons in the $30 \mathrm{keV}$ to $200 \mathrm{keV}$ range. The initial efforts to meet the wide scope of this goal have been to characterize the high energy $\mathrm{x}$-rays from undulator $\mathrm{A}$, measure the scattering from several liquid or amorphous materials for comparison to neutron data, and to characterize optics for high resolution Compton scattering.

A series of experiments were carried out to characterize the performance of the APS undulator $\mathrm{A}$ for delivering photons above $50 \mathrm{keV}$. The measured spectra from several different undulator gaps are shown in Fig. 4.48. The size of the monochromatic 
Table 4.6 Sector 1, Bending Magnet Beamline (1-BM)

\section{Responsible Scientists}

George Srajer, phone: (630) 252-3267, fax: (630) 252-0161, e-mail: srajerg @ aps.anl.gov

Jonathan Lang, phone: (630) 252-0122, fax: (630) 252-0161, e-mail: lang@ aps.anl.gov

\section{Scientific Programs}

- Polarization studies

- Magnetic scattering/spectroscopy

- Time resolved studies, including dispersive diffraction and dispersive spectroscopy

\section{Source Characteristics}

\begin{tabular}{|c|c|c|c|}
\hline \multicolumn{4}{|c|}{ Bending Magnet } \\
\hline \multicolumn{2}{|l|}{ Source size } & \multicolumn{2}{|c|}{$0.3 \times 0.3 \mathrm{~mm}^{2}$ (horiz. $\times$ vert.) FWHM } \\
\hline \multicolumn{2}{|l|}{ Source divergence } & \multicolumn{2}{|c|}{$6 \times 0.17 \mathrm{mrad}^{2}$ (horiz. $\times$ vert.) FWHM } \\
\hline \multicolumn{2}{|l|}{ Accepted horizontal divergence } & \multicolumn{2}{|c|}{$3.7 \mathrm{mrad}$} \\
\hline \multicolumn{2}{|l|}{ Power@100 mA } & \multicolumn{2}{|l|}{$87 \mathrm{~W} / \mathrm{mrad}$ (horiz.) } \\
\hline \multicolumn{4}{|l|}{ Optical Components } \\
\hline Component & $\begin{array}{l}\text { Distance from } \\
\text { Source }(\mathrm{m})\end{array}$ & Description & Status \\
\hline White beam slits & 23.0 & horiz. \& vert. & Operational \\
\hline Vertically collimating mirror & 25.0 & cylindrical bend & Installed 3/97 \\
\hline PSL double crystal monochromator & 27.5 & sagittal horizontal focus & Operational \\
\hline Dispersive crystal monochromator & 31.7 & meridional horizontal focus & Operational \\
\hline Monochromatic slits & 37.8 & horiz. \& vert. & Installed $12 / 96$ \\
\hline Vertically focusing mirror & 45.5 & cylindrical bend & To be installed fall 97 \\
\hline \multicolumn{4}{|c|}{$\begin{array}{l}\text { Beamline Configurations }(1-\mathrm{BM}) \\
\text { White Beam: }(1-\mathrm{BM}-\mathrm{B}) \\
\quad \text { beam at sample: } 5.5 \times 100 \mathrm{~mm}^{2}(\text { vert. } \times \text { horiz.) FWHM } \\
\text { Pink Beam: }(1-\mathrm{BM}-\mathrm{B}) \\
\quad \text { beam at sample: } 4.5 \times 100 \mathrm{~mm}^{2} \text { (vert. } \times \text { horiz.) FWHM, vertically collimated } \\
\text { Dispersive: }(1-\mathrm{BM}-\mathrm{B}) \\
\quad \text { beam at sample: } 4.5 \times 0.1 \mathrm{~mm}^{2} \text { (vert. } \times \text { horiz.) FWHM, E= } 6-20 \mathrm{keV}, \mathrm{dE} / \mathrm{E}=10^{-4}->10^{-1} \\
\text { Monochromatic: }(1-\mathrm{BM}-\mathrm{C}) \\
\quad \text { beam at sample: } 250 \times 100 \mu \mathrm{m}^{2} \text { (vert. } \times \text { horiz.) FWHM, E }=6-24 \mathrm{keV}, \mathrm{dE} / \mathrm{E}=10^{-4}\end{array}$} \\
\hline $\begin{array}{l}\text { Detectors } \\
\text { - NaI scintillation } \\
\text { - } \quad \text { Ionization chambers } \\
\text { Ancilli) solid-state detector } \\
\text { Ancillary Equipment } \\
\text { - Vacuum furnace } \\
\text { - } \text { 6-Circle diffractometer } \\
\text { - Polarimeter }\end{array}$ & & \multicolumn{2}{|c|}{$\begin{array}{l}\text { Beamline Control } \\
\text { - UNIX-based Sun workstations } \\
\text { - VME } \\
\text { - } \quad \text { EPICS } \\
\text { - } \\
\text { SPEC }\end{array}$} \\
\hline
\end{tabular}




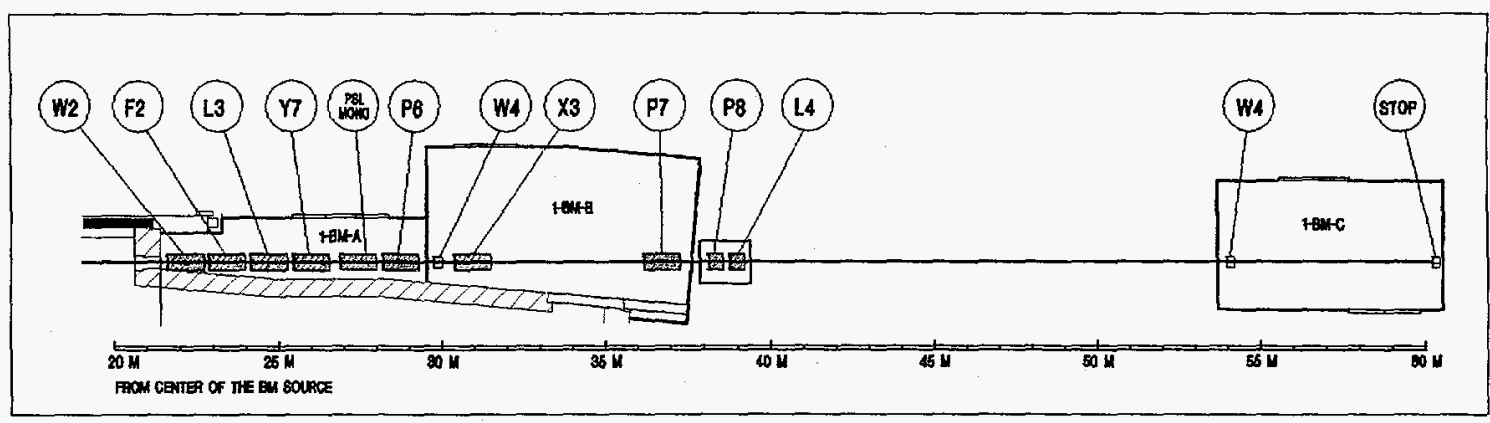

Fig. 4.47 Schematic layout of the Sector 1 BM beamline. Beam is traveling left to right.

W2: beryllium window, F2: filter, L3: white-beam slits, Y7: collimating mirror, PSL MONO: double-crystal monochromator, P6: movable white-beam stop and monochromatic shutter, W4: beryllium window, X3: bent-crystal monochromator, P7: white-beam stop and monochromatic shutter, P8: monochromatic shutter, LA: monochromatic slits, W4: beryllium window.

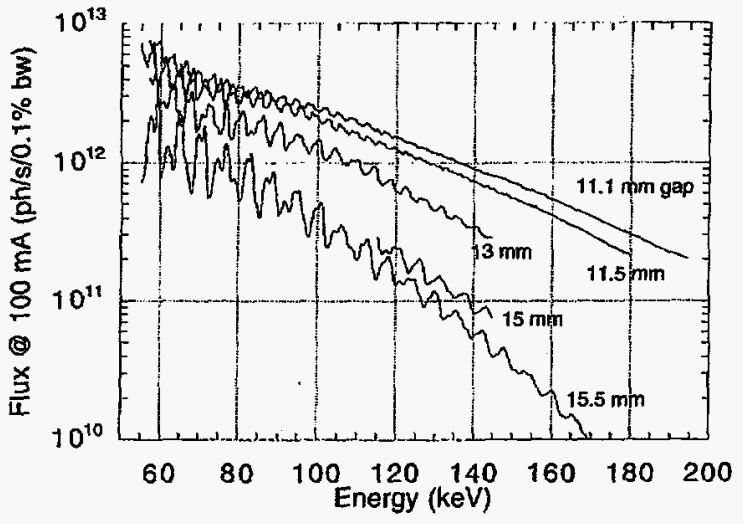

Fig. 4.48 APS undulator on-axis flux spectra through a $1 \mathrm{~mm} \times 1 \mathrm{~mm}$ aperture located 35 meters from the source.

beam was also measured for several energies at various undulator gaps. These results agree well with calculations made using the UR computer code (developed at the APS). Code UR uses the measured magnetic fields from actual devices in generating photon spectra. From the measurements it was found that undulator structure can be seen out to the 35 th harmonic and that at high energies the performance of the undulator approaches that of a low-K wiggler.
Among the characteristics that differentiate "high energy" $x$-rays from photons in the more commonly used $x$-ray energy range ( $6 \mathrm{keV}$ to $20 \mathrm{keV}$ ) is much lower absorption by materials and smaller diffraction angles for equivalent momentum transfer. Both of these attributes are utilized in the study of $\mathrm{x}$-ray scattering from amorphous/liquid materials. The absorption is comparable to that of neutrons for many materials, allowing the same samples and geometries to be used in both $\mathrm{x}$-ray and neutron studies. This greatly simplifies the task of comparing $x$-ray and neutron results for materials for which "nearsurface" effects are at issue. The low absorption also makes possible the study of materials inside relatively heavy containers (e.g., corrosive materials in stainless steel or samples at extreme temperatures and pressures). The small angles allow for the measurement of momentum transfer out to very large $Q$ (up to $20 \AA^{-1}$ ), which is needed for accurate structural determination.

A collaboration has been started with ANL/Materials Science Division scientists to use high energy $\mathrm{x}$-rays to examine amorphous glasses or liquids that have previously been 
studied with neutrons, but are difficult (if not impossible) to study with "ordinary" $\mathrm{x}$-rays. The first experiments have used $50 \mathrm{keV}$ and $150 \mathrm{keV}$ x-rays from 1-BM to examine molten $\mathrm{FeCl}_{3}$, molten $\mathrm{KPb}$, and $\mathrm{GeO}_{2}$ glass. For these experiments, the $\mathrm{FeCl}_{3}$ was contained in a tube of $\mathrm{SiO}_{2}$ and heated to $600 \mathrm{~K}$, and the $\mathrm{KPb}$ was in a vanadium can (as was used for neutron experiments) and heated to $800 \mathrm{~K}$. The normalized data for molten $\mathrm{FeCl}_{3}$ taken with both the neutrons and high energy $\mathrm{x}$-rays are shown in Fig. 4.49. Because the ratio of scattering from $\mathrm{Fe}$ to that from $\mathrm{Cl}$ is higher for $\mathrm{x}$-rays than for neutrons, it is quite easy to see that the second peak has more of a $\mathrm{Cl}$ contribution than the third peak. From detailed analysis, quantitative information is obtained about the partial structure factors.

Another aspect of the high energy program is to develop optics for high resolution Compton scattering. As part of this effort, a cryo-cooled DCM using $\mathrm{Si}$ (111) crystals set to diffract $86 \mathrm{keV}$ was assembled and put into 1-ID-B (86 keV is well outside the operating range of the Kohzu DCM). The monochromatic beam was then delivered to 1-ID-C to allow for data collection with very low background

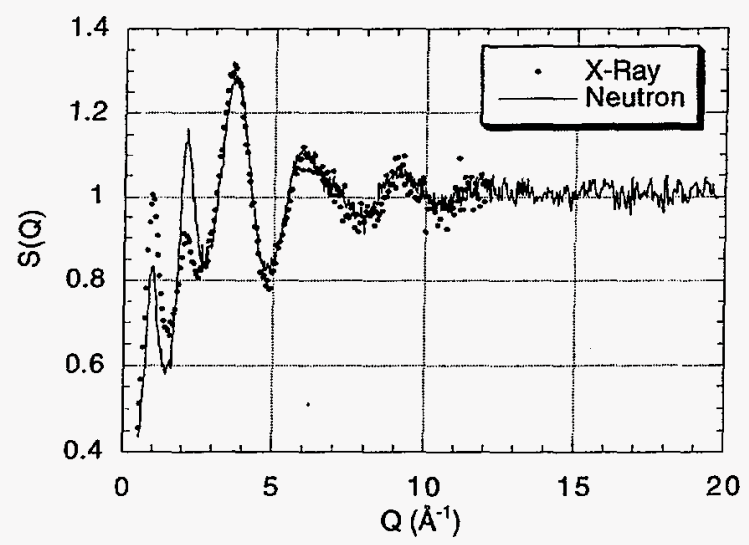

Fig. 4.49 Comparison of the scattering from molten $\mathrm{FeCl}_{3}$ at $600 \mathrm{~K}$ for $50 \mathrm{keV} x$-rays and neutrons. radiation. This beam was used to characterize both a scanning Laue analyzer and a Cauchois-type analyzer (used with image plates). Resolutions of approximately 0.1 a.u. were obtained for both systems. The next step in this effort will be to adapt the monochromator crystals to a bent Laue-Bragg system to increase throughput and focus the beam.

\section{Hard X-ray Polarization Program}

The main objective of the hard x-ray polarization program is the development of instrumentation and techniques for the production and analysis of polarized synchrotron radiation. Particular emphasis has been given to the design of phase-retarding crystal optics that yield the following characteristics:

- high degree of circular polarization

- high degree of stability

- tunability in a wide energy range

- rapid helicity switching

To accomplish this, phase retarders have been developed for two energy regimes: low energies ( $5 \mathrm{keV}-30 \mathrm{keV})$ and high energies $(>30 \mathrm{keV})$. For low energies, helicityswitching phase retarders made of thin $(50 \mu \mathrm{m}$ to $1000 \mu \mathrm{m}$ ) single-crystal diamond or silicon have been tested (see Fig. 4.50). These phase retarders are suitable for magnetic circular dichroism or resonant-magnetic-scattering experiments. For high energies, a new helicity-switching phase retarder designed to operate at $86 \mathrm{keV}$ has been manufactured and 


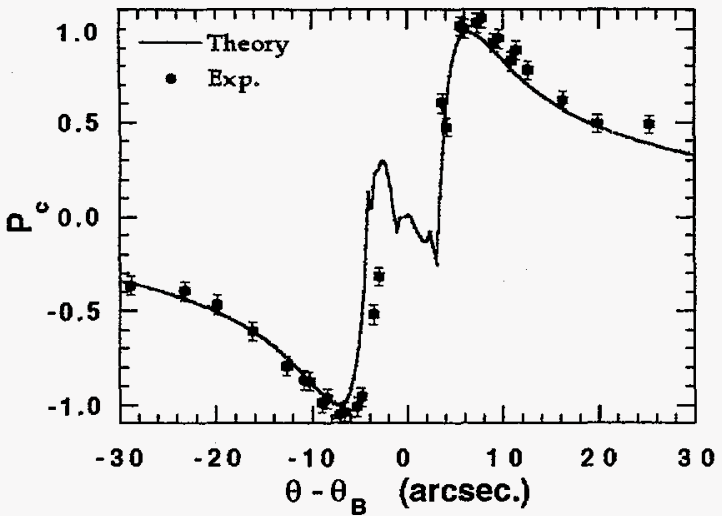

Fig. 4.50 Measured and theoretical circular polarization for the transmitted beam in a 75-mm-thick Si (400) crystal at $8.0 \mathrm{keV}$.

successfully tested (see Fig. 4.51). The high energy phase retarder is made of a single, monolithic germanium crystal in the BraggLaue design. With further improvements of this phase retarder in mind, work on the development of a broad ( $1 \mathrm{keV})$ bandpass high energy monochromator is currently in progress. These monochromators are made of W/Si bilayers with a goal of $~ 90 \%$ reflectivity at $100 \mathrm{keV}$. For polarization analysis, a polarimeter utilizing multibeam diffraction was built and tested. This device enables complete characterization of polarization for energies up to $15 \mathrm{keV}$.

To utilize and further test the low energy, phase-retarding optics developed at the APS, a series of temperature-dependent dichroism experiments have been performed. The goal of these experiments was to measure the different exchange couplings present in rare earthtransition metal alloys. An example of the results is shown in Fig. 4.52, which shows the temperature dependence of the dichroism in $\mathrm{HoFe}_{2}$. The different temperature dependencies of the lower negative feature and higher positive feature are directly correlated with the magnetization of the Ho $4 \mathrm{f}$ and $5 \mathrm{~d}$ bands. An understanding of the exchange

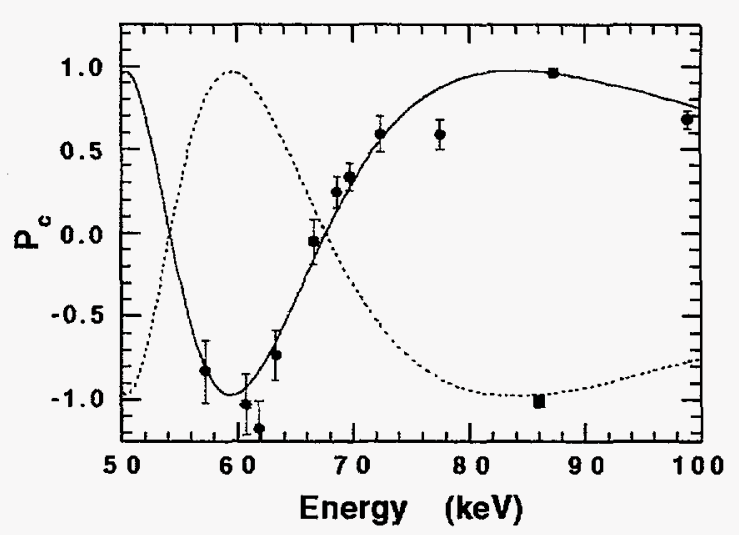

Fig. 4.51 Measured and theoretical rate of circular polarization for the (220) and (2-20) beams as a function of energy. The phase retarder is designed to operate at $86.5 \mathrm{keV}$.

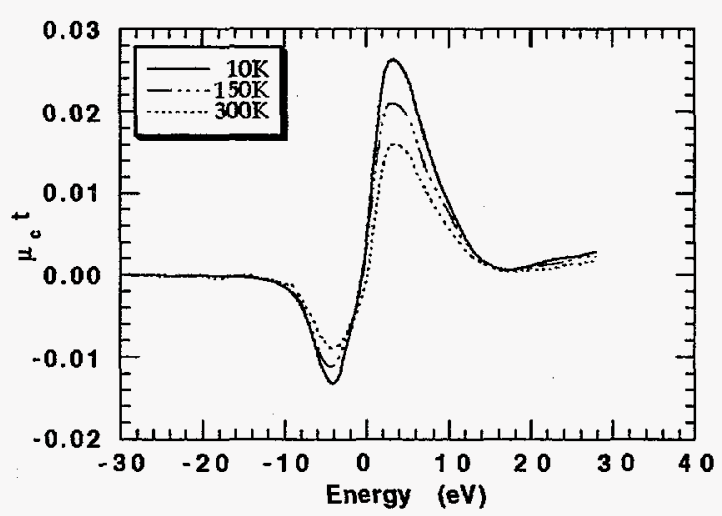

Fig. 4.52 Variations in the dichroic spectrum as a function of temperature at the $\mathrm{Ho}_{3}$ edge of $\mathrm{HoFe}_{2}$.

couplings that control the magnetization of the $5 \mathrm{~d}$ band is essential in the development of new magnetic materials. The use of phase-retarding optics proved essential in this experiment due to the difficulty of reversing the sample magnetization in cryogenic environments.

As a continuation of the study of magnetic systems utilizing circularly polarized hard $\mathrm{x}$-rays, preliminary experiments on the magnetic properties of thin films have commenced and a program exploring 
spin-dependent Compton scattering has been initiated.

\section{Time-Resolved Studies}

The primary goal of the time-resolved program is the development of instruments and techniques for time-resolved $\mathrm{x}$-ray studies. The primary $\mathrm{x}$-ray optic associated with the time-resolved program is the dispersive $\mathrm{BCM}$ located in the 1-BM-B station. The $\mathrm{BCM}$ provides a polychromatic beam with a distinct relation between angle and beam energy such that when used with spatially resolved detectors (see below), time-resolved EXAFS or dispersive-diffraction spectra can be collected. The BCM has been designed to operate in an energy range between 6 and $20 \mathrm{keV}$, typical for absorption edges of interest in EXAFS studies. The horizontal spot size produced by the BCM has been measured to be $\sim 150$ microns at $11.0 \mathrm{keV}$ with a measured flux of $1.5 \times 10^{11} \mathrm{ph} / \mathrm{s} /$ $100 \mathrm{~mA}$.

An integral part of the time-resolved program is the development of high speed area detectors. For time-resolved $\mathrm{x}$-ray scattering, charge coupled device (CCD) detectors with multiple outputs are the best choice for high speed, reasonable-size applications. Current commercially available detectors have frame rates comparable to that of TV signaling rates, 30 frames per second (fps). Typically these cameras can digitize each pixel at up to 10 bits (1024 light levels). At slower frame rates (typically less than $1 \mathrm{fps}$ ), it is possible to digitize each pixel to 16 bits. These cameras are not adequate for the types of experiments we envision for the APS, hence we have pursued a program for the development of a high framing rate CCD-based camera system.
The camera system that has been developed here is composed of a camera head unit and a data collection unit. The camera head unit uses a CCD as the $\mathrm{x}$-ray imager. It contains all of the circuitry necessary to control the CCD and digitize each pixel. The data collection unit consists of a digital interface capable of storing high speed digital data. The camera was designed for a multiple output CCD with a maximum pixel rate of $20 \mathrm{MHz}$ per output. This gives a 2-output CCD with an area of $512 \times 512$ pixels a readout time of about 10 milliseconds (ms), corresponding to a maximum of about $100 \mathrm{fps}$. The camera digitizes each pixel to 12 bits (one of 4096 light levels). The data rate from this camera will be up to 80 megabytes per second. The data collection unit must capture and store this data. The approach used is to store the data in an intermediate storage area before they are written to a disk drive of a computer so that they can be manipulated, analyzed, and permanently archived. This intermediate storage area is conventional dynamic random access memory (DRAM). The acquisition unit can be designed in two ways. The data can be written directly to a DRAM storage card and then read out to a computer and stored to a hard disk drive. For time-resolved $\mathrm{x}$-ray imaging, the camera is used with a phosphor screen to convert $\mathrm{X}$-ray photons to visible light that is then imaged onto the CCD. This imaging is either done by lenses (for large area imaging) or by means of a fiber-optic face plate or taper directly bonded to the CCD. The phosphor screen in this case is coupled by means of optical gel to the fiber-optic element. The camera is designed so that is can also be used as a streak camera where one or several rows of data are read out continuously achieving time resolutions of few tens of microsecond. The camera system has been also tested using a $1024 \times 1024$ pixel $C C D$ from Texas Instruments (TC215) that is read out from two outputs as a $512 \times 512$ device 
with four pixel hardware binning. A camera currently being designed is based upon a Thomson TH7896 $(1024 \times 1024,4$ output $)$. After the engineering issues of a 4-output camera are solved, a camera will be developed using a 16-output $512 \times 512$ Sarnoff CCD capable of 800 frames/second.

To date, the camera system has been used as a diagnostic tool in the commissioning of the dispersive monochromator on the 1-BM beamline. The camera system was used to collect the $\mathrm{Cu}$ absorption spectrum in 10 milliseconds (shown in Fig. 4.53) and has been configured to be used as a streak camera, measuring the absorption of copper with a time resolution of 50 microseconds.

\subsubsection{Sector 2}

Sector 2 consists of an ID beamline (2-ID) with three branches and a BM beamline (2-BM). At Sector 2, there are five scientific programs distributed among four experiment end stations:

- High resolution microfocusing and microscopy (2-ID-B, 2-ID-D)

- Coherence and correlation techniques (2-ID-B, 2-ID-D)

- High resolution spectroscopy and polarization physics (2-ID-C)

- X-ray optics characterization and technique development (2-BM-B)

- Deep x-ray lithography (2-BM-B)

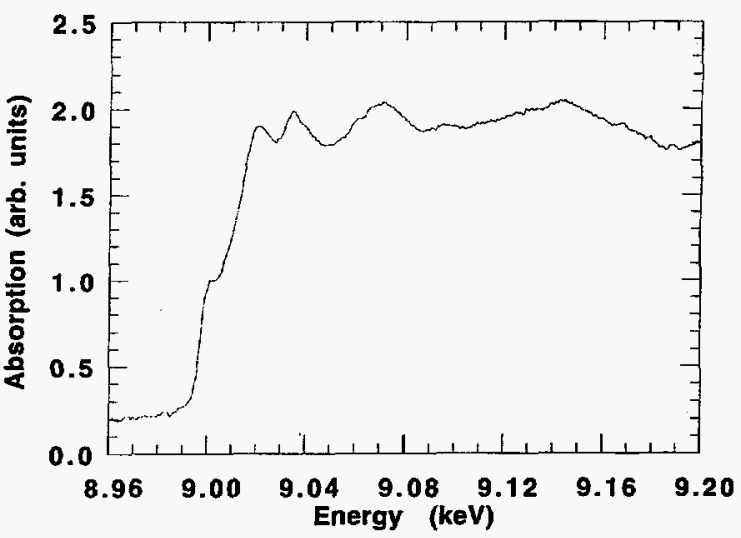

Fig. $4.53 \mathrm{Cu}$ EXAFS spectrum taken in $10 \mathrm{~ms}$ using the dispersive monochromator.

\section{Beamline Descriptions}

To accomplish the objectives of the Sector 2 scientific programs, the first- and third-order harmonics of two 2.4-m-long undulators (a standard 3.3-cm-period APS undulator A and a special 5.5-cm-period undulator) are used to cover the $0.5-32 \mathrm{keV} x$-ray energy range on the 2-ID beamline. The 2-BM source has a critical energy of $19.5 \mathrm{keV}$ and provides broadband $\mathrm{x}$-rays over the $0.5-32 \mathrm{keV}$ region. Details of the source, optics, and beamline design can be found in the Sector 2 Preliminary Design Report, Final Design Report, and elsewhere (Yun et al., 1996a, 1996b; Shu et al., 1996; McNulty et al., 1996; Lai et al., 1996). A brief description is given here.

\section{2-ID}

The 2-ID-A station FOE houses various apertures and optics to handle the high incident power and manipulate the incident $\mathrm{x}$-ray beam. The three branch lines share the FOE but have independent experiment 
stations, 2-ID-B， 2-ID-C，2-ID-D/E (see Fig. 4.54). The three branch lines also share a water-cooled, high heat load, horizontally deflecting mirror (M1) that operates at a grazing incidence angle of $0.15^{\circ}$. The pink beam following $\mathrm{M} 1$ has a maximum cutoff energy of $32 \mathrm{keV}$. The branching is achieved inside 2-ID-A using horizontally deflecting mirrors: a second mirror (M2B or M2C, depending on the branch line in operation), and a third mirror (M3B). The other components in 2-ID-A include a fixed aperture, an adjustable aperture, a bremsstrahlung collimator, a white-beam stop, a BPM, and beam shutters for each branch line. These components are permanent parts of the beamline that remain in place when switching between branch lines.

The 2-ID-B branch line is designed for the $0.5-4 \mathrm{keV}$ energy range. It receives the beam reflected successively by the $\mathrm{M} 1, \mathrm{M} 2 \mathrm{~B}$, and $\mathrm{M} 3 \mathrm{~B}$ mirrors and is oriented at an angle of $5.0^{\circ}$ to the 2-ID-D/E branch. Branch line 2-ID-B uses a horizontally deflecting spherical grating monochromator (SGM) consisting of an entrance slit, a grating chamber holding up to five diffraction gratings, an exit slit, a differential pump, and a silicon nitride exit window inside an environmentally isolated experimental enclosure. The enclosure provides acoustic and temperature control for sensitive experiments. The branch line has three BPMs for beam monitoring and feedback.

The 2-ID-C branch line operates over the $0.5-3 \mathrm{keV}$ range. It receives the beam reflected successively by the $\mathrm{M} 1$ and $\mathrm{M} 2 \mathrm{C}$ mirrors at an angle of $2.5^{\circ}$ to the $2-\mathrm{ID}-\mathrm{D} / \mathrm{E}$ branch. Branch line 2-ID-C has a vertically deflecting third mirror (M3C, located outside of 2-ID-A) for

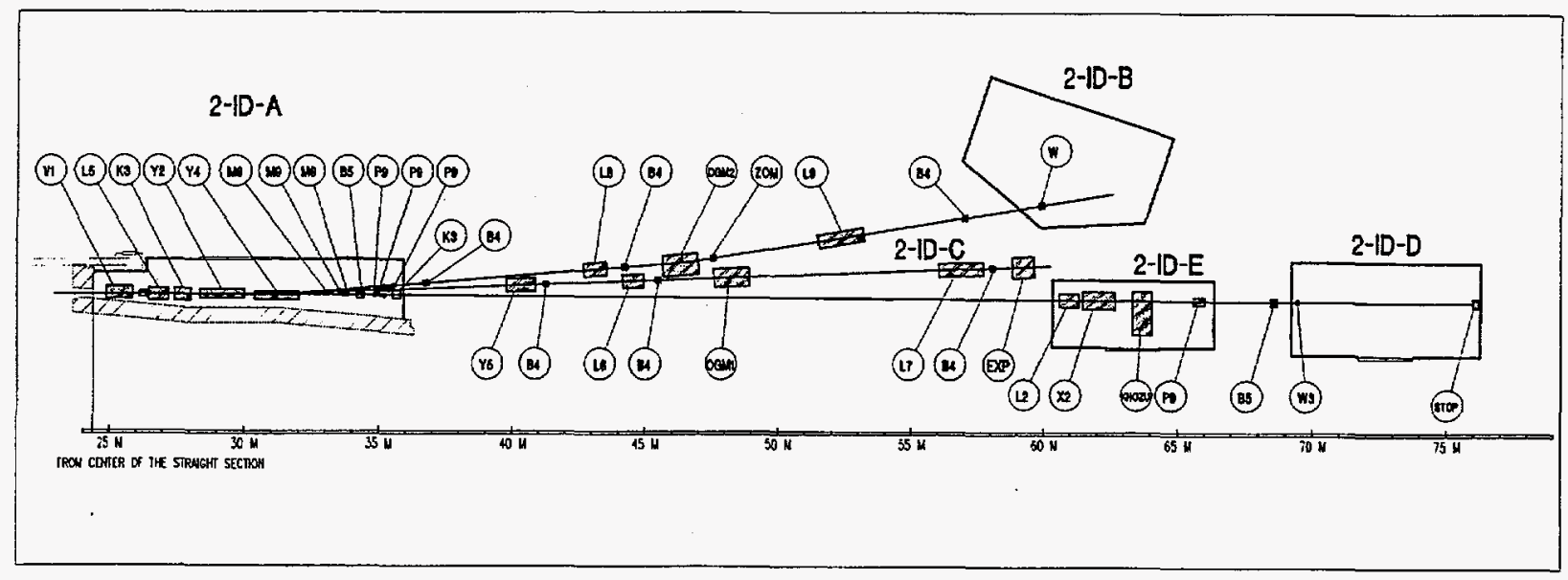

Fig. 4.54 Schematic of beamline 2-ID. The labeled components in 2-ID-A are: V1, differential pump and cooled aperture; L5, adjustable slits; K3, bremsstrahlung collimator; $Y 2$, high heat load horizontally deflecting mirror (M1); Y4, soft $x$-ray horizontally deflecting beam-branching mirrors (M2C, M2B, M3B); M9, cooled masks; B5, beam position monitor; P9, branch line shutters; K3, collimator. The branch line and experiment station components are: B4, beam position monitor; Y5, vertically focusing mirror; $L 6$ and L8, entrance slits; L7 and L9, exit slits, OGM1, vertically deflecting SGM; OGM2, horizontally deflecting SGM; ZOM, zero-order mask; $W$, silicon nitride window; EXP, soft $x$-ray spectroscopy station; L2, adjustable slits; X2, doublemultilayer monochromator; Kohzu, DCM; P9, shutter; B5, beam position monitor; W3, beryllium window; STOP, beam stop. 
vertical focusing. Mirror $\mathrm{M} 3 \mathrm{C}$ deflects the beam downward by $2^{\circ}$. The $2-\mathrm{ID}-\mathrm{C}$ branch line uses a vertically deflecting SGM optimized for high spectral resolution. The experiment station following the exit slit consists of a UHV experimental chamber. Branch line 2-ID-C also has three BPMs.

The 2-ID-D/E branch line is designed to cover 4-32 keV and has two experiment stations, 2-ID-E and 2-ID-D. The 2-ID-E station is located at $\sim 30 \mathrm{~m}$ downstream of $2-\mathrm{ID}-\mathrm{A}$ and houses pink-beam slits, a double-multilayer monochromator, a beryllium window, a DCM, and a pink-beam shutter. The main experiment station, 2-ID-D, can receive pink or monochromatic beam through a final beryllium window.

The three branch lines cannot be operated simultaneously in the current design. However, switching of the beam from one branch to another can be completed in a few minutes. A significant portion of the experiments planned for the branch lines may be performed with little compromise by beam sharing. A summary of the beamline hardware is shown in Tables 4.7, 4.8, and 4.9.

\section{2-BM}

The 2-BM beamline has two experiment stations, 2-BM-A and 2-BM-B (see Fig. 4.55). Station 2-BM-A (which is also serves as a FOE) contains a mirror and two monochromators to satisfy the different bandwidth requirements of the experimental program. A DCM is used for experiments requiring narrow band $\mathrm{x}$-rays, whereas a double-multilayer monochromator provides broad band monochromatization. The other components in 2-BM-A include a fixed mask, differential pumping station, filter assembly, white-beam slits, bremsstrahlung collimator, beryllium window, and a shutter.

The 2-BM-B station is located $\sim 20 \mathrm{~m}$ further downstream. It can receive either the monochromatic or pink beam from 2-BM-A. It

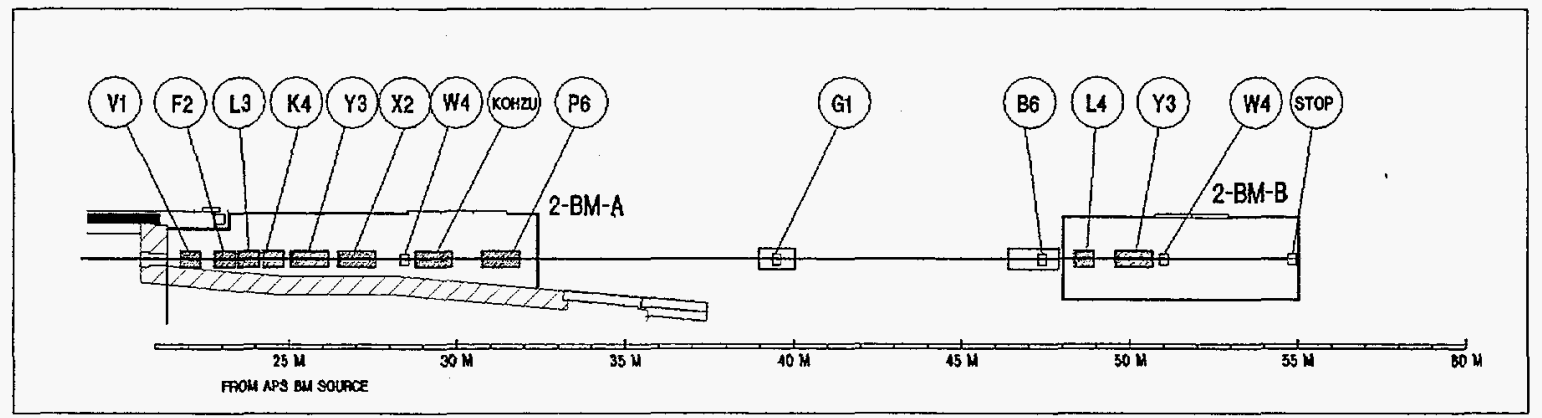

Fig. 4.55 Schematic of beamline 2-BM. The components in 2-BM-A are: VI, differential pump and cooled aperture; F2, selectable filters; L3, adjustable slits; $K 4$, bremsstrahlung collimator; Y3, vertically deflecting mirror (MI); X2, double-multilayer monochromator; W4, beryllium window; Kohzu, DCM; P6, shutter. Following 2-BM-A are: G1, diagnostic screen; B6, beam position monitor; LA, adjustable slits; Y3, vertically deflecting mirror (M2) W4, beryllium window; STOP, beam stop. 
Table 4.7 Sector 2, Insertion Device Branch Line (2-ID-B)

\section{Responsible Scientists}

Ian McNulty, phone: (630) 252-2882, fax: (630) 252-9303, e-mail: mcnulty@ aps.anl.gov

Yiping Feng, phone: (630) 252-1081, fax: (630) 252-9303, e-mail: yfeng@aps.anl.gov

\section{Scientific Programs}

- High resolution imaging

- Coherent scattering

- Interferometry

\section{Source Characteristics}

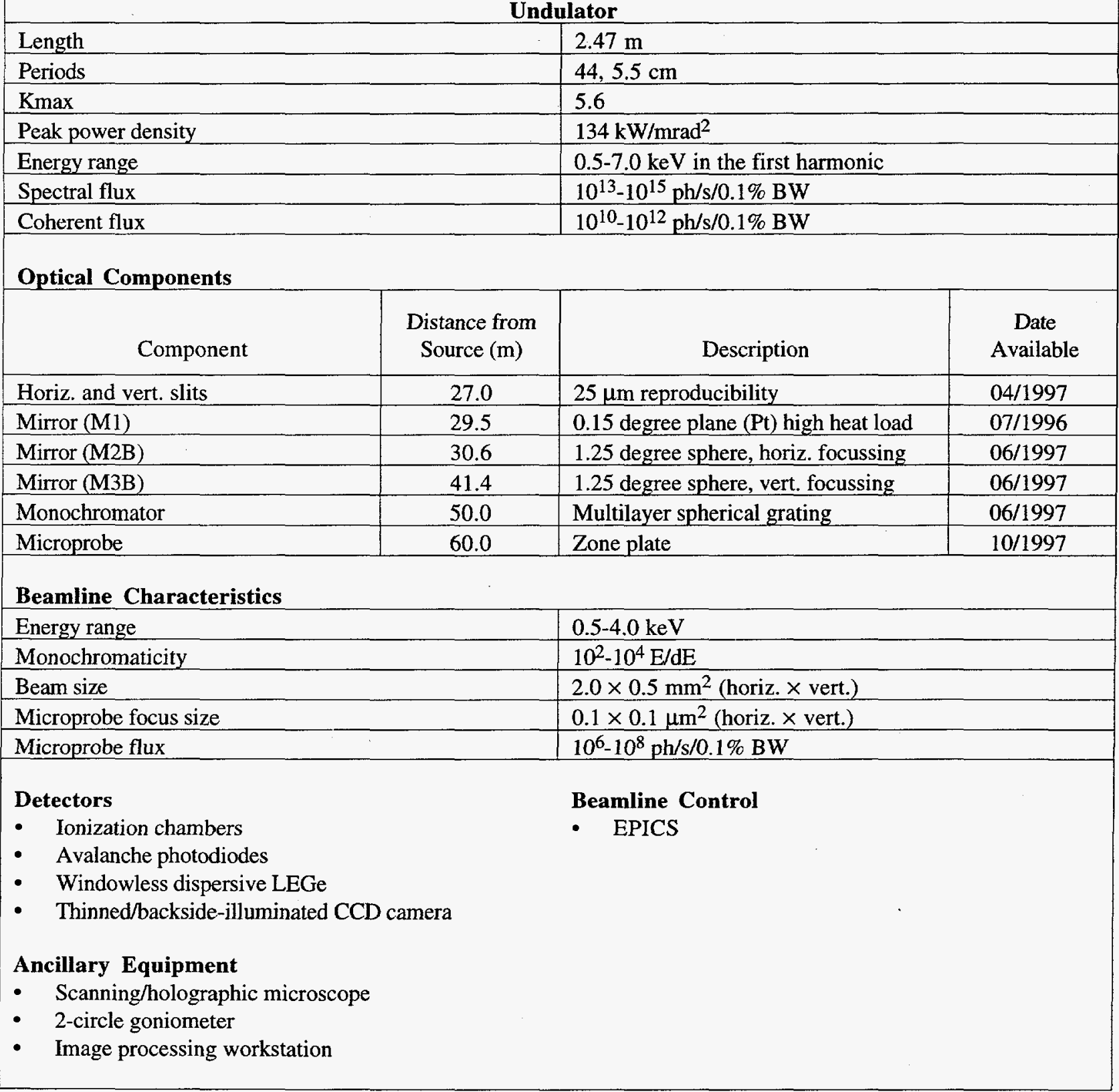




\section{Table 4.8 Sector 2, Insertion Device Branch Line (2-ID-C)}

\section{Responsible Scientists}

Kevin J. Randall, phone: (630) 252-9614, fax: (630) 252-9303, e-mail: randall@ aps.anl.gov

Joseph Z. Xu, phone: (630) 252-0143, fax: (630) 252-0161, e-mail: jzxu@aps.anl.gov

\section{Scientific Programs}

- High resolution polarization-dependent spectroscopy

\section{Source Characteristics}

\begin{tabular}{|c|c|c|c|}
\hline \multicolumn{4}{|c|}{ Undulator } \\
\hline \multicolumn{2}{|l|}{ Length } & \multicolumn{2}{|l|}{$2.47 \mathrm{~m}$} \\
\hline \multicolumn{2}{|l|}{ Periods } & \multicolumn{2}{|l|}{$44,5.5 \mathrm{~cm}$} \\
\hline \multicolumn{2}{|l|}{$\mathrm{Kmax}$} & \multicolumn{2}{|l|}{5.6} \\
\hline \multicolumn{2}{|l|}{ Peak power density } & \multicolumn{2}{|l|}{$134 \mathrm{~kW} / \mathrm{mrad}^{2}$} \\
\hline \multicolumn{2}{|l|}{ Energy range } & \multicolumn{2}{|l|}{$0.5-7.0 \mathrm{keV}$ in the first harmonic } \\
\hline \multicolumn{2}{|l|}{ Spectral flux } & \multicolumn{2}{|l|}{$10^{13}-10^{15} \mathrm{ph} / \mathrm{s} / 0.1 \% \mathrm{BW}$} \\
\hline \multicolumn{2}{|l|}{ Coherent flux } & \multicolumn{2}{|l|}{$10^{10}-10^{12} \mathrm{ph} / \mathrm{s} / 0.1 \% \mathrm{BW}$} \\
\hline \multicolumn{4}{|l|}{ Optical Components } \\
\hline Component & $\begin{array}{l}\text { Distance from } \\
\text { Source (m) }\end{array}$ & Description & $\begin{array}{c}\text { Date } \\
\text { Available }\end{array}$ \\
\hline Horiz. and vert. slits & 27.0 & $25 \mu \mathrm{m}$ reproducibility & $04 / 1997$ \\
\hline Mirror (M1) & 29.5 & 0.15 degree plane $(\mathrm{Pt})$ high heat load & $07 / 1996$ \\
\hline Mirror (M2C) & 30.6 & 1.25 degree sphere, horiz. focussing & $06 / 1997$ \\
\hline Mirror (M3C) & 41.4 & 1.00 degree sphere, vert. focussing & $10 / 1997$ \\
\hline Monochromator & 50.0 & Multilayer spherical grating & $10 / 1997$ \\
\hline Experiment station & 60.0 & UHV sample chamber & $10 / 1997$ \\
\hline \multicolumn{4}{|l|}{ Beamline Characteristics } \\
\hline \multicolumn{2}{|l|}{ Energy range } & \multicolumn{2}{|l|}{$0.5-3.0 \mathrm{keV}$} \\
\hline \multicolumn{2}{|l|}{ Monochromaticity } & \multicolumn{2}{|l|}{$10^{3}-10^{4} \mathrm{E} / \mathrm{dE}$} \\
\hline \multicolumn{2}{|l|}{ Beam size at sample } & \multicolumn{2}{|l|}{$1.0 \times 1.0 \mathrm{~mm}^{2}$ (horiz. $\times$ vert.) } \\
\hline \multicolumn{2}{|l|}{ Flux at sample } & \multicolumn{2}{|l|}{$10^{11}-10^{13} \mathrm{ph} / \mathrm{s}$} \\
\hline \multicolumn{2}{|l|}{$\begin{array}{l}\text { Detectors } \\
\text { - } \quad \text { Hemispherical electron/ion analyzer } \\
\text { - } \quad \text { Photodiodes } \\
\text { - } \quad \text { Windowless } \mathrm{Si}(\mathrm{Li}) \text { detector } \\
\text { - Total electron yield detector } \\
\text { Ancillary Equipment } \\
\text { - UHV reflectometer } \\
\text { - UHV polarimeter } \\
\quad 100 \mathrm{~nm} \text { electron gun }\end{array}$} & \multicolumn{2}{|l|}{$\begin{array}{l}\text { Beamline Control } \\
\text { - EPICS }\end{array}$} \\
\hline
\end{tabular}


Table 4.9 Sector 2, Insertion Device Branch Line (2-ID-D/E)

\begin{tabular}{|c|c|c|c|}
\hline \multicolumn{4}{|c|}{$\begin{array}{l}\text { Responsible Scientists } \\
\text { Wenbing Yun, phone: (630) 252-5 } \\
\text { Zhonghou Cai, phone: (630) 252-0 } \\
\text { Scientific Programs } \\
\text { - High resolution imaging } \\
\text { - Coherence-based techniques } \\
\text { Source Characteristics }\end{array}$} \\
\hline \multicolumn{2}{|l|}{ Length } & \multicolumn{2}{|l|}{$2.47 \mathrm{~m}$} \\
\hline \multicolumn{2}{|l|}{ Periods } & \multicolumn{2}{|l|}{$74,3.3 \mathrm{~cm}$} \\
\hline \multicolumn{2}{|l|}{ Kmax } & \multicolumn{2}{|l|}{2.6} \\
\hline \multicolumn{2}{|l|}{ Peak power density } & \multicolumn{2}{|l|}{$170 \mathrm{~kW} / \mathrm{mrad}^{2}$} \\
\hline \multicolumn{2}{|l|}{ Energy range } & \multicolumn{2}{|l|}{$3.2-13.0 \mathrm{keV}$ in the first harmonic } \\
\hline \multicolumn{2}{|l|}{ Spectral flux } & \multicolumn{2}{|l|}{$10^{12}-10^{14} \mathrm{ph} / \mathrm{s} / 0.1 \% \mathrm{BW}$} \\
\hline \multicolumn{2}{|l|}{ Coherent flux } & \multicolumn{2}{|l|}{$10^{10}-10^{12} \mathrm{ph} / \mathrm{s} / 0.1 \% \mathrm{BW}$} \\
\hline \multicolumn{4}{|l|}{ Optical Components } \\
\hline Component & $\begin{array}{l}\text { Distance from } \\
\text { Source }(\mathrm{m})\end{array}$ & Description & $\begin{array}{c}\text { Date } \\
\text { Available }\end{array}$ \\
\hline Horiz. and vert. slits & 27.0 & $25 \mu \mathrm{m}$ reproducibility & $04 / 1997$ \\
\hline Mirror (M1) & 29.5 & 0.15 degree plane $(\mathrm{Pt})$ high heat load & $07 / 1996$ \\
\hline Double crystal mono. & 62.0 & Kohzu, Si (111) & $12 / 1996$ \\
\hline Double multilayer mono. & 63.7 & Medium resolution & $10 / 1997$ \\
\hline Microprobe & 71.0 & Zone plate & $12 / 1996$ \\
\hline \multicolumn{4}{|l|}{ Beamline Characteristics } \\
\hline \multicolumn{2}{|l|}{ Energy range } & \multicolumn{2}{|l|}{$2.0-32.0 \mathrm{keV}$} \\
\hline \multicolumn{2}{|l|}{ Monochromaticity } & \multicolumn{2}{|l|}{$1.0-10^{4} \mathrm{E} / \mathrm{dE}$} \\
\hline \multicolumn{2}{|l|}{ Beam size } & \multicolumn{2}{|l|}{$4.2 \times 1.6 \mathrm{~mm}^{2}$ (horiz. $\times$ vert.) FWHM } \\
\hline \multicolumn{2}{|l|}{ Microprobe focus size } & \multicolumn{2}{|l|}{$0.1 \times 0.1 \mu \mathrm{m}^{2}$ (horiz. $\times$ vert.) } \\
\hline \multicolumn{2}{|l|}{ Microprobe flux } & \multicolumn{2}{|l|}{$10^{11} \mathrm{ph} / \mu \mathrm{m}^{2} / \mathrm{s} / 0.1 \% \mathrm{BW}$} \\
\hline \multicolumn{2}{|c|}{$\begin{array}{l}\text { Detectors } \\
\text { - Ionization chambers } \\
\text { - NaI scintillation detector } \\
\text { - Avalanche photodiodes } \\
\text { - CCD camera } \\
\text { - } 13 \text {-element energy-dispersive detector } \\
\text { Ancillary Equipment } \\
\text { - Optical tables with } 6 \text { degrees of freedom } \\
\text { - Scanning microscope } \\
\text { - High resolution } x \text {-ray spectrometer } \\
\text { Image processing workstation }\end{array}$} & \multicolumn{2}{|l|}{$\begin{array}{l}\text { Beamline Control } \\
\text { - EPICS }\end{array}$} \\
\hline
\end{tabular}


contains a beam position monitor, monochromatic beam slits, and a beryllium exit window. In 2-BM-B there is also a mirror that is used as a broad band energy selector. The required spectral range (e.g., for lithography experiments) can be adjusted by varying the incidence angle of the mirror. Table 4.10 provides a summary of the beamline characteristics and hardware.

\section{Current status}

Construction and commissioning of Sector 2 is about $70 \%$ complete. Both experiment stations of 2-BM have been commissioned; 2-BM-A awaits a double-multilayer monochromator, the last major component to be installed in 2-BM. Most of the components in the 2-ID-A FOE and the 2-ID-D/E branch line have been commissioned. The final component to be installed in $2-\mathrm{ID}-\mathrm{A}$ is the $\mathrm{M} 2 \mathrm{C} / \mathrm{M} 2 \mathrm{~B} / \mathrm{M} 3 \mathrm{~B}$ mirror manipulator and branch-line switching system. A doublemultilayer monochromator and integral shutter remain to be installed in 2-ID-E, whereas the 2-ID-D station is complete. The 2-ID-B and 2-ID-C branch lines and end stations are under construction and to be commissioned in mid1997.

\section{Scientific Program}

\section{2-ID}

The main objective of the 2-ID scientific program is to develop experimental techniques and instrumentation that utilize the high spectral brilliance, large transverse coherence widths, and low energy polarization properties of the APS undulator beams. These include high resolution $\mathrm{x}$-ray microfocusing, spectroscopy, coherence, and correlation experiments. In addition to these areas, the 2-ID beamlines will be used to develop diagnostic techniques for measuring the source emittance, characterizing the absolute flux, intensity, and low energy polarization of the beam, and for developing high performance $x$-ray optics (mirrors, multilayers, crystals, and gratings) to preserve the brilliance and low energy polarization of the undulator beam under high heat load conditions.

\section{High resolution microfocusing and microscopy}

The 2-ID-B and 2-ID-D/E branch lines provide undulator radiation with the spectral and coherence properties required for development of high resolution $x$-ray microfocusing optics and related techniques, such as scanning microscopy, phase-contrast microscopy, microdiffraction, microimaging, and microanalysis in the intermediate and high energy ranges from $0.5-4$ and $4-32 \mathrm{keV}$, respectively. Consequently these branch lines are optimized for brilliance and coherence preservation, high order harmonic rejection, and angular stability.

Several experiments have been carried out since the commissioning of the 2-ID-D station in December 1996. Selected results are presented below.

(1) Study of an electro-absorption laser/ modulator using $\mathrm{x}$-ray microdiffraction. In collaboration with a team from Lucent Technologies, we have made spatial maps of the crystallographic strain, composition, and multiquantum well thickness of an integrated laser/modulator device, one of the key components in long-haul lightwave 
Table 4.10 Sector 2, Bending Magnet Beamline (2-BM)

\footnotetext{
Responsible Scientists

Scientific Programs

- Development of $\mathrm{x}$-ray optics

- Beam diagnostic instrumentation

- Deep x-ray lithography

- X-ray microprobe studies

\section{Source Characteristics}

Barry Lai, phone: (630) 252-6405, fax: (630) 252-9303, e-mail: blai@aps.anl.gov

Derrick C. Mancini, phone: (630) 252-0147, fax: (630) 252-0161, e-mail: mancini@aps.anl.gov

\begin{tabular}{|c|c|c|c|}
\hline \multicolumn{4}{|c|}{ Bending Magnet } \\
\hline \multicolumn{2}{|l|}{ Source size } & \multicolumn{2}{|l|}{$0.3 \times 0.3 \mathrm{~mm}^{2}$ (horiz. $\times$ vert.) FWHM } \\
\hline \multicolumn{2}{|l|}{ Source divergence } & \multicolumn{2}{|l|}{$6 \times 0.17 \mathrm{mrad}^{2}$ (horiz. $\times$ vert.) FWHM } \\
\hline \multicolumn{2}{|l|}{ Accepted horizontal divergence } & \multicolumn{2}{|l|}{$2.0 \mathrm{mrad}$} \\
\hline \multicolumn{2}{|l|}{ Power@100 mA } & \multicolumn{2}{|l|}{$87 \mathrm{~W} / \mathrm{mrad}$ (horiz.) } \\
\hline \multicolumn{4}{|l|}{ Optical Components } \\
\hline Component & $\begin{array}{l}\text { Distance from } \\
\text { Source }(\mathrm{m})\end{array}$ & Description & $\begin{array}{c}\text { Date } \\
\text { Available }\end{array}$ \\
\hline Filter assembly & 23.2 & 8 filters on 2 carriers & $05 / 1996$ \\
\hline Horiz. and vert. slits & 23.5 & $25 \mu \mathrm{m}$ reproducibility & $05 / 1996$ \\
\hline Vert. deflecting mirror & 24.9 & 0.15 degree plane w $/ 2$ coatings $(\mathrm{Cr}, \mathrm{Pt})$ & $07 / 1997$ \\
\hline Double multilayer mono. & 27.4 & Unfocussed & $10 / 1997$ \\
\hline Double crystal mono. & 28.8 & Kohzu, unfocussed & $04 / 1997$ \\
\hline Horiz. and vert. slits & 48.3 & $25 \mu \mathrm{m}$ reproducibility & $02 / 1997$ \\
\hline Vert. deflecting mirror & $\begin{array}{c}49.4 \\
(24.9 \mathrm{~m}, 06 / 1996)\end{array}$ & Variable angle $(0-2$ degrees) plane $(\mathrm{Pt})$ & $08 / 1997$ \\
\hline
\end{tabular}

\section{Beamline Configurations}

Pink Beam: (2-BM-B)

beam at sample: $4 \times 100 \mathrm{~mm}^{2}$ (horiz. $\times$ vert.), uncollimated

Monochromatic (multilayer): (2-BM-B)

beam at sample: $4 \times 100 \mathrm{~mm}^{2}$ (horiz. $\times$ vert.), $\mathrm{E}=0.5-33 \mathrm{keV}, \mathrm{dE} / \mathrm{E}=10^{-2}$

Monochromatic (crystal): (2-BM-B)

beam at sample: $4 \times 100 \mathrm{~mm}^{2}$ (horiz. $\times$ vert.), $\mathrm{E}=2.5-33 \mathrm{keV}, \mathrm{dE} / \mathrm{E}=10^{-4}$

\section{Detectors}

- Energy dispersive solid state detector

- Ionization chambers

- Scintillation detector

\section{Ancillary Equipment}

- Precision scanning stage for $\mathrm{x}$-ray lithography

- Micropositioning system

- Diffractometer

\section{Beamline Control}

- EPICS 
transmission systems. The device is an InGaAsP-based monolithic quantum well laser and modulator, which has an active region about $1 \mathrm{~mm}$ wide. The microbeam is essential for mapping the strain field in a buried device with such dimensions. The strain field in the laser and modulator, and the transition region between them, has important consequences for device performance. The strain is measured by taking a Bragg scan $(\theta / 2 \theta)$ along the growth direction and determining the position of the zeroth order quantum-well superlattice peak (Fig. 4.56). The quantum well and barrier thickness are determined from the spacing of the superlattice peaks and their relative intensities. This information is very useful for guiding the device fabrication process.

(2) Study of the plant-fungi relationship in contaminated environments. The objective of this project is to study the relationship between plants and mycorrhizal fungi in soils contaminated by heavy metals and radionuclides. An understanding of the role of mycorrhizal fungi in the adaptation of plants to contaminated environments and the elucidation of the uptake and sequestration mechanisms for heavy metal and radionuclides in these

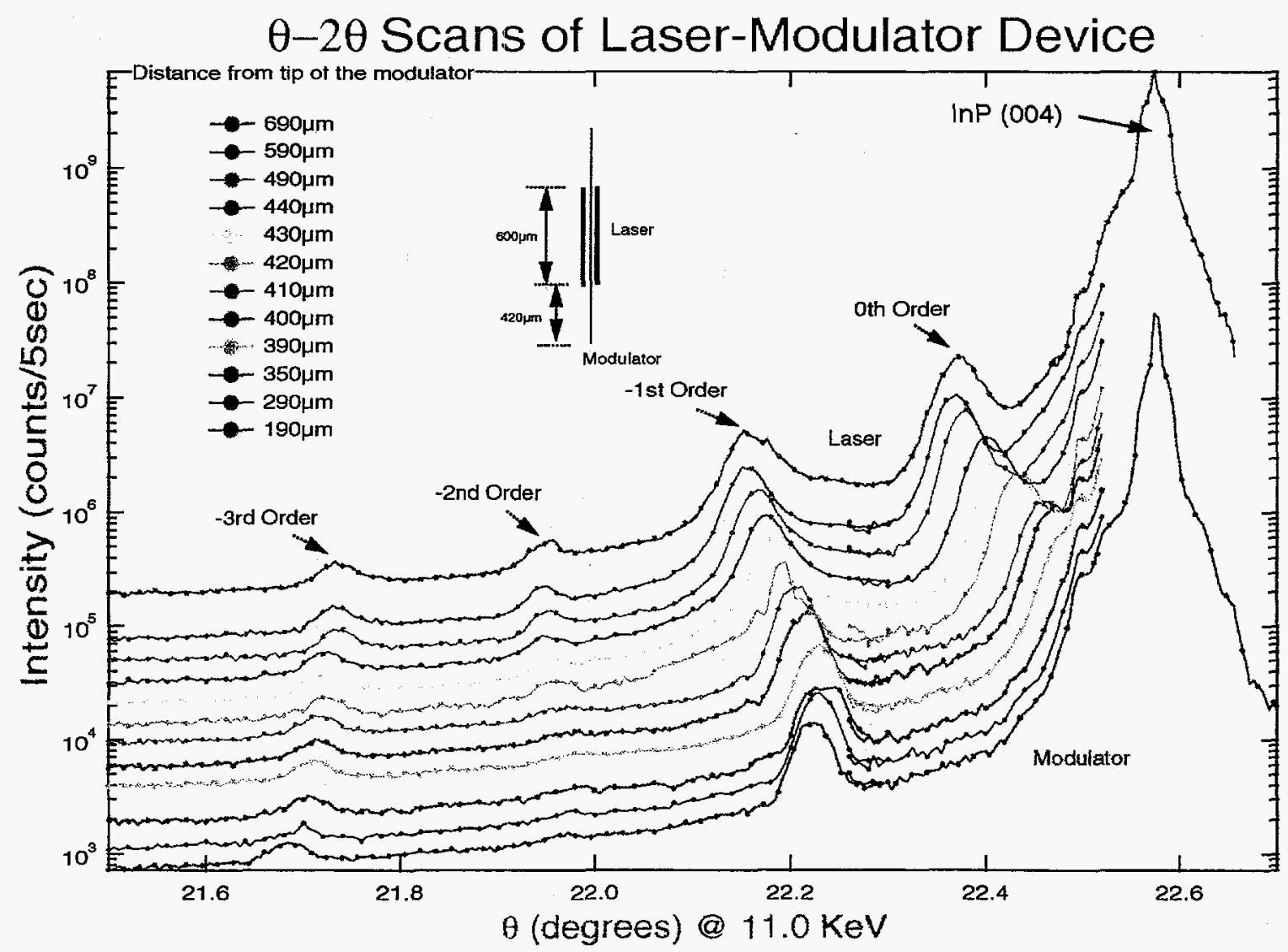

Fig. 4.56 Radial Bragg scans along the In (004) substrate direction taken at several positions. The focal spot was about $1 \mu \mathrm{m}(V) \times 3 \mu \mathrm{m}(H)$. The variation in multiquantum well period and strain as a function of position is observed as a variation in spacing between superlattice peaks (labeled by order) and position of the zeroth-order peak, respectively. 
systems are expected to have important ramifications both in the remediation and restoration of waste sites and in the assessment of risk associated with such sites.

$\mathrm{X}$-ray fluorescence microscopy was used for mapping elemental distributions of several metallic elements, including $\mathrm{Ca}, \mathrm{Cr}, \mathrm{Fe}, \mathrm{Zn}$, $\mathrm{Cu}$, and $\mathrm{K}$. We were able to detect trace elements in a single fungi strand with diameters of about $10 \mu \mathrm{m}$. Using x-ray phase contrast imaging, we were able to record images of a single strand. With improved detector resolution and efficiency, a phase contrast microscope with $1-\mu \mathrm{m}$ resolution and the sensitivity to detect submicron-thick biological samples can be developed. The combination of this capability with the elemental sensitivity may prove to be an extremely useful tool for biological research.

(3) X-ray microdiffraction study of fiberreinforced materials. Fabrication-induced residual stress in engineered composites consisting of continuous fibers may result in large tensile hoop stresses at the fiber/matrix interface, sufficient to cause plastic deformation or radial cracking of the matrix in low ductility systems. An understanding of the residual and operational stress field near the interface between the matrix and fiber is of great importance to designers of components as well as materials engineers responsible for developing processes for fabricating high quality composites.

Using $\mathrm{x}$-ray microdiffraction techniques, we mapped the strain field in a composite material system consisting of $\mathrm{SiC}$ fibers in a $\mathrm{Ti} / \mathrm{Al} / \mathrm{Nb}$ host matrix. Preliminary analysis of the data indicates that good agreement was obtained between the measured strained field and that calculated using a simple continuum model (Fig. 4.57). The high spatial resolution $(10 \mu \mathrm{m})$ is essential for this measurement because the strain field changes rapidly over a region of $50 \mu \mathrm{m}$.

\section{Coherence and correlation techniques}

The 2-ID-B and 2-ID-D/E branch lines are also dedicated to development of $\mathrm{x}$-ray coherence-based and correlation techniques such as holography, interferometry, speckle, and correlation spectroscopy. Both branch lines offer selectable energy bandwidth and spatial coherence of the beam in order to satisfy the needs of a wide variety of coherence dependent experiments.

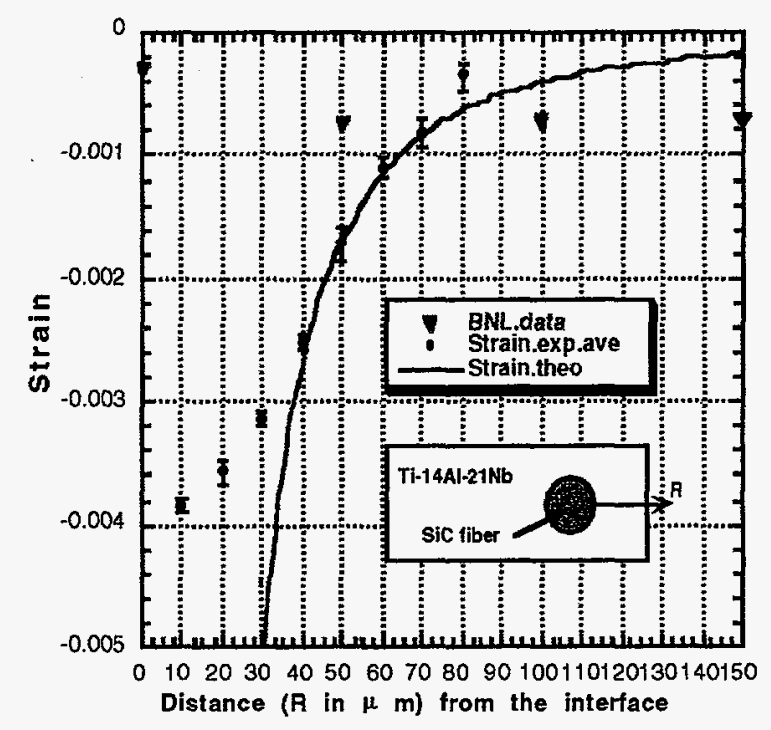

Fig. 4.57 Measured strain field using our $x$-ray microscope (dots) at the APS, measured strain using a $50 \mu \mathrm{m}$ pinhole at the NSLS (triangles), and the calculated strain field (solid line) using a using a simple continuum model. The residual strain is significantly less than theoretically predicted because of plastic relaxation and radial cracking during sample cooling. 
Real-time observation of x-ray fluctuations can provide valuable information about the structural dynamics of a wide range of systems including metallic alloys, glasses, complex fluids, and biological specimens. $\mathrm{X}$-ray photon correlation spectroscopy (or $\mathrm{x}$-ray intensity fluctuation spectroscopy) has been demonstrated to be a promising technique for studying such dynamics. For this type of experiment, an intense highly coherent $\mathrm{x}$-ray beam is required. We tested the setup for a new method of measuring correlated $\mathrm{x}$-ray fluctuations, by $\mathrm{x}$-ray heterodyne correlation spectroscopy, at 2-BM-A in December 1996. In February 1997, we installed and tested it at 2-ID-D. In the first phase of the experiment, we tested the stability of the beam using two collinear pinholes and monochromatic beam (Si (111)). The autocorrelation function of the direct beam intensity passing through the second pinhole was measured, indicating the angular beam stability was better than $0.5 \mu \mathrm{rad}$, once a good orbit was established. In the second phase, Fraunhofer diffraction patterns were measured for 5 - $\mu \mathrm{m}$-diameter pinholes by both scanning a $5 \mu \mathrm{m}$ analyzing pinhole and using a CCD camera. More than twenty fringes were resolved in the $\mathrm{CCD}$ image, indicating high spatial coherence of the beam at the pinhole location and that our pinholes were of high quality. Fraunhofer patterns with good visibility were obtained from pinhole diameters up to $20 \mu \mathrm{m}$, indicating that the spatial coherence width of the incident beam was of this scale. Static speckle patterns were then measured from a Vycor glass sample for 5,10 and $20 \mu \mathrm{m}$ source pinholes (Fig. 4.58). The speckle size and visibility was found to change accordingly. In the final phase, pink beam was used. The $\mathrm{Pt}$ pinhole used proved to be robust for sustaining the pink beam, and optical microscopy showed no damage resulted from the high incident $\mathrm{x}$-ray flux. We

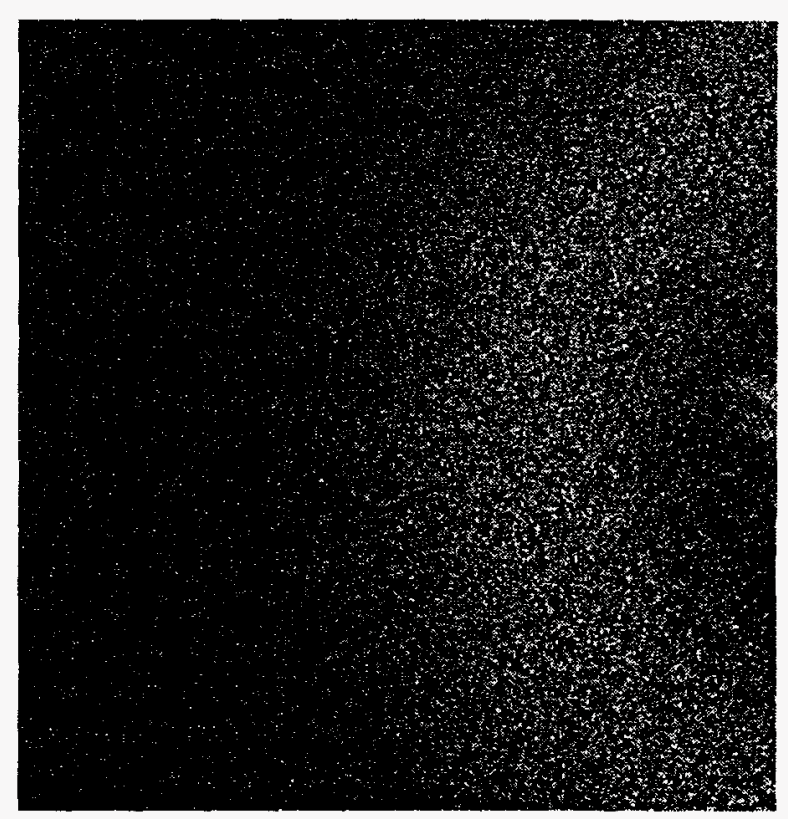

Fig. 4.58 Speckle pattern from coherent scattering by a porous Vycor glass. The image was captured by a CCD camera at $1.6 \mathrm{~m}$ using a $5 \mathrm{~mm}$ Pt source pinhole.

are now ready to perform dynamic studies during the next available beam time.

We also demonstrated $\mathrm{x}$-ray fluorescence fluctuation spectroscopy for the first time. This is a novel technique that combines high source brilliance and microfocusing to study number fluctuations as a function of time. Because illumination of a very small volume is required in order to measure the number fluctuation of a specific element in the sample, we used a zone plate to focus the incident beam to $0.6 \mu \mathrm{m} \times 1 \mu \mathrm{m}$. We successfully observed the fluctuating fluorescence signal of a gold colloid sample and calculated its autocorrelation function, from which the dynamic information was obtained. Both the translation and rotation diffusion constants were measured. This technique has the virtue of elemental specificity and may find application in studies of material and biological systems. 
High resolution spectroscopy

The 2-ID-C branch line is dedicated to polarization-dependent spectroscopy and instrument development in the intermediate energy range from 0.5 to $3 \mathrm{keV}$. In order to preserve the beam polarization and to achieve high flux throughput simultaneously at high resolving power, the 2-ID-C branch line uses a grazing incidence SGM. The experiment station offers a UHV experimental chamber with separate analysis and preparation sections. This chamber has a high efficiency electron/ion energy analyzer, LEED system, electron gun with a beam size adjustable to less than $1000 \AA$, ion sputter gun, thin film evaporation system with piezo thickness monitor, and a precision sample manipulator.

The spectroscopy program includes surface science studies of the magnetic properties of thin films and multilayers containing transition metals and rare earth compounds. A major part of the program is devoted to studying the properties of these structures using angle, spin and spatially resolved photoemission, as well as by resonant scattering. Atoms and simple molecules will be studied both in gas phase, and as surface adsorbates, primarily with the electron/ion analyzer. High detection efficiency of the analyzer will enable resolution of weak nondipole or satellite features. It will also be possible to make correlated electronelectron, electron-ion or electron-photon measurements using coincidence techniques.

\section{Source diagnostics}

As part of SRI-CAT's mission to develop and characterize new sources, we have made considerable progress in 1996 measuring the absolute flux, coherent flux, source size and source divergence of the $3.3-\mathrm{cm}$-period undulator A at 2-ID (Cai et al., 1996a, 1996b).

In addition, the spectrum of undulator $\mathrm{A}$ was measured for two tapered-gap configurations: (a) upstream gap $=18.5 \mathrm{~mm}$, downstream gap $=19.5 \mathrm{~mm}$; (b) upstream gap $=18.5 \mathrm{~mm}$, downstream gap $=21.5 \mathrm{~mm}$. While the measured spectrum agrees well with that calculated using XOP code (Dejus \& Sanchez del Rio, 1996) for the untapered case, the spectra for the tapered cases do show significant discrepancies. We are currently looking into this.

\section{2-BM}

The 2-BM scientific program focuses on development of deep x-ray lithography and development of techniques and instrumentation in support of the Sector 2 beamlines.

\section{Optics characterization and technique development}

Also under development at 2-BM are instrumentation, diagnostics, and optical schemes and techniques for high brilliance ID and BM sources, and $x$-ray optics testing and characterization including reflectivity, scattering, and diffraction measurements on mirrors, multilayers, crystals and gratings, zone plates, and other focusing elements. These capabilities support the Sector 2 scientific programs and enable more efficient utilization of the shared time on the three branch lines of 2-ID.

During commissioning, experiments were conducted in the 2-BM-A station. The FOE is not suitable for some measurements such as 
scattering due to the higher background. Other experiments were conducted, and the highlights follow:

- Microtomography experiments using a high spatial resolution $\mathrm{CCD}$ camera were performed on microstructures of interest. The data collection of 90 views within a $180^{\circ}$ range was completed within half an hour. The reconstruction demonstrated a very high spatial resolution of $1-2 \mu \mathrm{m}$ in all three directions (Fig. 4.59).

- The focusing efficiency of blazed zone plates with a 3 -step profile were measured to be $38-44 \%$. This is considerably higher than the $25 \%$ efficiency measured on conventional binary zone plates with a single-step profile. Also, the background intensity
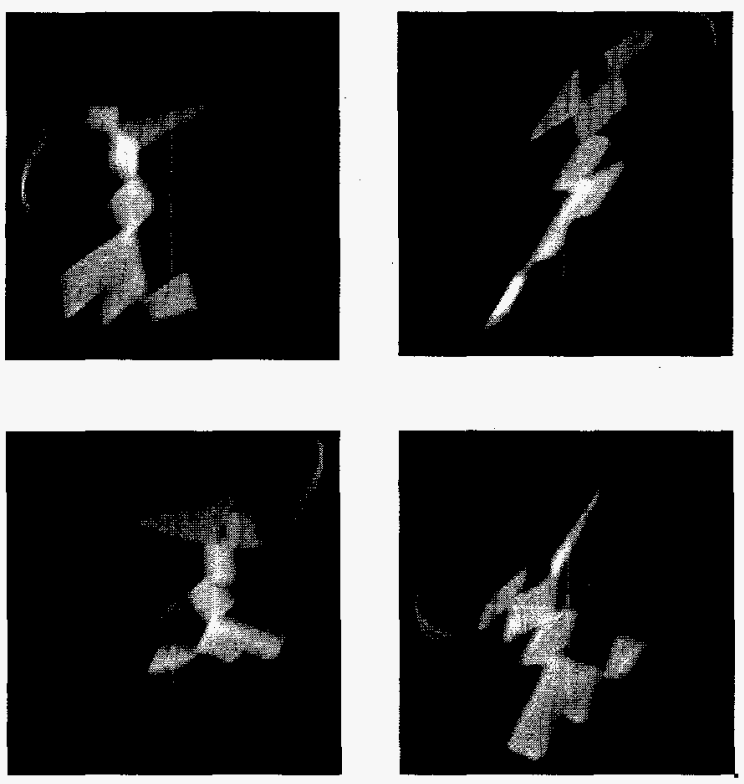

Fig. 4.59 Reconstruction of a gold microstructure imaged with microtomography. The gold squares are $100 \mu \mathrm{m} \times 100 \mu \mathrm{m} \times 10 \mu \mathrm{m}$ in size. The snapshots show the bent and twisted shape of the square chain. from zero and high orders in the case of blazed zone plates is much suppressed relative to the first order $(<1: 100)$, which is critical for high sensitivity microanalysis measurements.

- Instrumentation for heterodyne correlation spectroscopy were tested. The feasibility of the concept and the experimental setup were verified. The quality of the entrance pinhole was confirmed by measuring its diffraction effects.

- The reflectivity and scattering from a superpolished $\mathrm{Al}$ substrate coated with electroless $\mathrm{Ni}$ (intended for $\mathrm{x}$-ray astronomy applications) were measured. These data were compared with AFM and WYCO roughness measurements to assess their validity at a different spatial frequency region.

- The reflectivity of the first mirror was measured, and it agrees with the theoretical reflectivity within $5 \%$ from 10 to $35 \mathrm{keV}$. The reflectivities of several candidate multilayers for the monochromator were measured and the filter foils in the filter assembly were characterized by transmission.

\section{Deep x-ray lithography}

A key objective at 2-BM is to develop deep $\mathrm{x}$-ray lithography (DXRL) at energies above $1 \mathrm{keV}$. Deep $\mathrm{x}$-ray lithography is uniquely capable of fabricating high precision microstructures with very high aspect ratios (height/lateral dimension) (Ehrfield \& Münchmeyer, 1991; Ehrfield \& 
Lehr, 1994). This technique will be exploited to fabricate diffractive optical elements such as zone plates and gratings, slits and pinholes, and other components such as micromechanical parts.

With a high critical energy and a highly collimated beam, the APS is well suited for producing high-aspect-ratio microstructures in thick resist films (>1 mm) using DXRL. The 2-BM beamline was designed to exploit these benefits and to advance the capabilities of DXRL. Initial applications include $\mathrm{x}$-ray optical elements and advanced accelerating structures. The process of fabricating microstructures using DXRL is shown schematically in Fig. 4.60. The x-ray mask consists of patterns defined by an absorbing material (usually $\mathrm{Au}$ ) supported on a thin plate (Be, Si, etc.). The pattern is then transferred to the resist layer using $\mathrm{X}$-ray proximity exposure. A widely used photoresist is

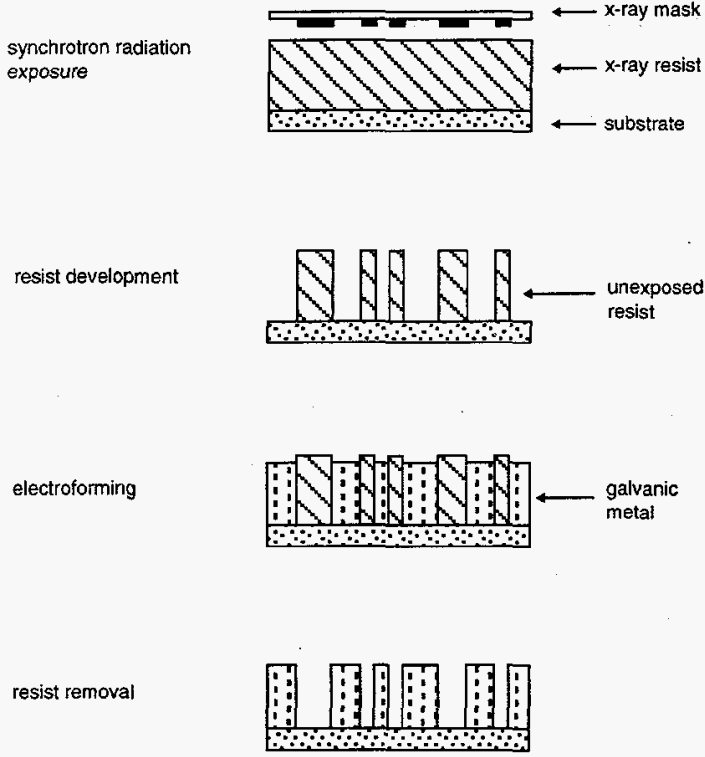

Fig. 4.60 Schematic of the DXRL process: exposure, resist development, electroforming, and resist stripping. Note the final structure has a higher aspect ratio than the mask. polymethylmethacrylate (PMMA). To obtain a uniform exposure, the mask and resist are scanned together through the $\mathrm{x}$-ray beam in the vertical plane. After exposure, the resist is developed in organic solvent, the final structures are electroformed onto the substrate, and the remaining resist is stripped away. This pattern transfer process is highly precise, and the aspect ratio of the final structure can be considerably enhanced relative to the original mask.

Lithography exposures had been performed in the FOE, using a prototype scanner developed for use during the initial phase. Using this scanner, initial characterization of 1- and 2.5-mm-thick PMMA resists at different $x$-ray spectra, dose rate, total dose, and developer conditions was performed. A more advanced scanner that can better utilize the source characteristics and enables exposures of inclined or nonplanar structures has been designed and specified. An EPICS-based exposure control module has been developed to integrate the scanner operation with other beamline controls and to automate the exposure.

Beryllium is a promising substrate material for the x-ray mask, providing better mechanical stability and thermal conductivity than silicon, which is critical for handling the high thermal load at the APS. Beryllium masks with patterned gold structures were fabricated, and their radiation stability in the $x$-ray beam was studied. Figure 4.61 shows structures in a developed 1-mm-thick PMMA resist exposed with the beryllium mask. The patterns are part of a prototype coded aperture for x-ray astronomy application, fabricated in collaboration with NASA Goddard Space Flight Center. Also, fabrication and initial exposure for mm-wave accelerating cavity structures have been done together with the APS/ASD rf group. 


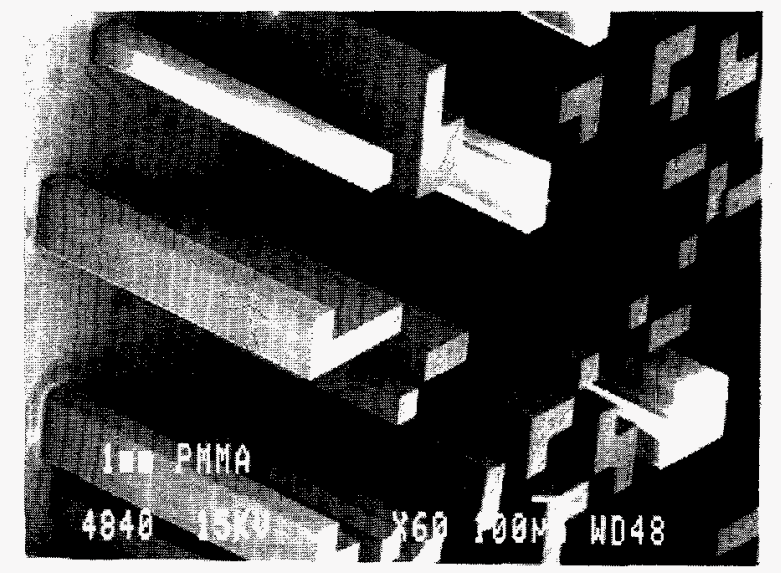

Fig. 4.61 Scanning electron micrograph of structures of coded aperture patterns in a 1-mm-thick PMMA resist.

\section{Future R\&D Plans}

The 2-BM beamline will be completed in late 1997 with the installation of a collimating mirror, multilayer monochromator for broad bandpass experiments, and a 6-circle diffractometer for microdiffraction applications. The mirror will provide a collimated beam with uniform intensity, which will enable us to fabricate more precise microstructures and provide better spatial resolution in microfocusing applications. A microfocusing setup will also be installed in the experiment station. Two enhancements will be added to the lithography scanner: a sample rotation stage for fabricating conical structures, and an interferometric feedback system. A mask-tosubstrate aligner will be developed and integrated with the scanner to enable precise field stitching and multiple step exposure. A processing cleanroom will be built to provide mask fabrication, resist processing, electroforming, and sample metrology capabilities.

Final commissioning activities at 2-ID-B will include installation and characterization of a real-time BPM system. Deep multilayer gratings, which offer substantially higher efficiency, will be fabricated and tested in the 2-ID-B monochromator. A high-harmonicrejection and beam steering system based on a double-bounce mirror will also be developed to attenuate high undulator harmonics passed by the monochromator. The 2-ID-B scanning microscope software will be enhanced, enabling XANES imaging, microtomography, microfluorescence, and microdiffraction experiments. We will explore possibilities for real-time microtomography using both high performance workstations and an on-site teraflop supercomputer.

The UHV photoemission station at 2-ID-C will be used for surface science, gas phase, thin film and multilayer measurements. The UHV goniometer will be used for polarization-dependent studies of thin films and multilayers using plane polarized radiation. We will also develop a zone-platebased microprobe system and CCD detector for photoemission and fluorescence spectromicroscopy with both systems. A mini-Mott electron spin polarimeter will be commissioned and used for photoemission measurements of magnetic samples. Several external SRI-CAT members will use their own experimental equipment at 2-ID-C, e.g., an angle-resolving photoemission station for photoelectron holography (T.-C. Chiang, University of Illinois), and energy dispersive secondary emission fluorescence analyzer (Dave Ederer, Tulane University).

At 2-ID-D/E, a 13-element detector, wavelength dispersive spectrometer, large-format CCD camera, and precision conical slits will be added to the instrumentation supporting the hard x-ray microscope. Further integration of the microscope software and hardware will be completed for routine operation of the system by general users. In support of the high resolution x-ray optics development program, a high speed sputtering system for producing 
zone plates for high energy (40-150 keV) applications will be designed and procured. Related instruments, such as a polisher, slicer, and laser system for accurately measuring the diameter of sputtered-slice zone plates, will be developed.

Because of the long lead times required for manufacturing x-ray mirrors, we will begin development of a high heat load $\mathrm{x}$-ray mirror for operation with $300 \mathrm{~mA}$ current in the storage ring. This mirror will replace the high heat load mirror (M1) currently used in our beamline.

We are developing a new technique for microfocusing $x$-ray beams from a high brilliance source using thin-film waveguides. The method consists of using the evanescent wave produced in a thin cover plate for the waveguide (designed for total reflection of the incident beam) to excite a resonant mode in the guide, thereby coupling most of the incident beam intensity into the region between the plane surfaces of the waveguide, which can be microfabricated to be as small as $20 \mathrm{~nm}$. The method has already been demonstrated by us previously in a preliminary experiment at the ESRF (Feng et al., 1995). The technique will also be used to study the structure and dynamics of fluids placed inside the waveguide.

We are also developing a technique for measuring residual stress in components machined of materials ranging from $\mathrm{Be}$ to $\mathrm{U}$, using the microdiffraction capabilities of the instrumentation at station 2-ID-D/E, and the capability of microfocusing $\mathrm{X}$-ray beams with energies over $100 \mathrm{keV}$ currently under development. The method should yield a 3-D map of the texture and strain in the sample, and could be used, for instance, to image the stress distribution around a crack tip.

\subsubsection{Sector 3}

The Sector 3-ID beamline is dedicated to high energy resolution $x$-ray scattering studies in the energy range of $6-30 \mathrm{keV}$, with a energy bandpass of $10^{-5}<\Delta \mathrm{E} / \mathrm{E}<10^{-13} \mathrm{eV}$. The development of optical components in terms of monochromators, analyzers, and detectors is heavily emphasized in the early years of operation. Along these lines, novel monochromator concepts are being introduced and tested.

The 3-ID beamline was optimized for the inelastic $\mathrm{X}$-ray scattering and nuclear resonant scattering programs. The 5-meter-long straight section for the beamline has room for two IDs that can be alternatively operated. Currently, a special 2.7-cm-period undulator, optimized for peak brilliance around $14 \mathrm{keV}$ is installed, with the option of adding undulator $\mathrm{A}$ when energies out of the range of the $2.7-\mathrm{cm}$-period undulator are required. The layout of this undulator beamline is given in Fig. 4.62. The FOE, 3-ID-A, covers the area of the beamline immediately downstream of the ratchet-wall penetration and is used to house various components that manipulate the $\mathrm{x}$-ray beam prior to transmitting the beam downstream to the experiment stations. The components in 3-ID-A are permanent parts of the beamline and include filters, white-beam slits, the Kohzu DCM, monochromatic-beam shutters, and a bremsstrahlung collimator/stop. A beryllium window separates the monochromator from the FE (i.e., the beamline inside the ratchet wall) and, hence, the storage-ring vacuum. The 3-ID-B station is located directly downstream of 3-ID-A and houses the mirror and high resolution optics.

The high resolution monochromator consists of two high angular resolution stages (12.5 nanoradians/step) attached to vertical 


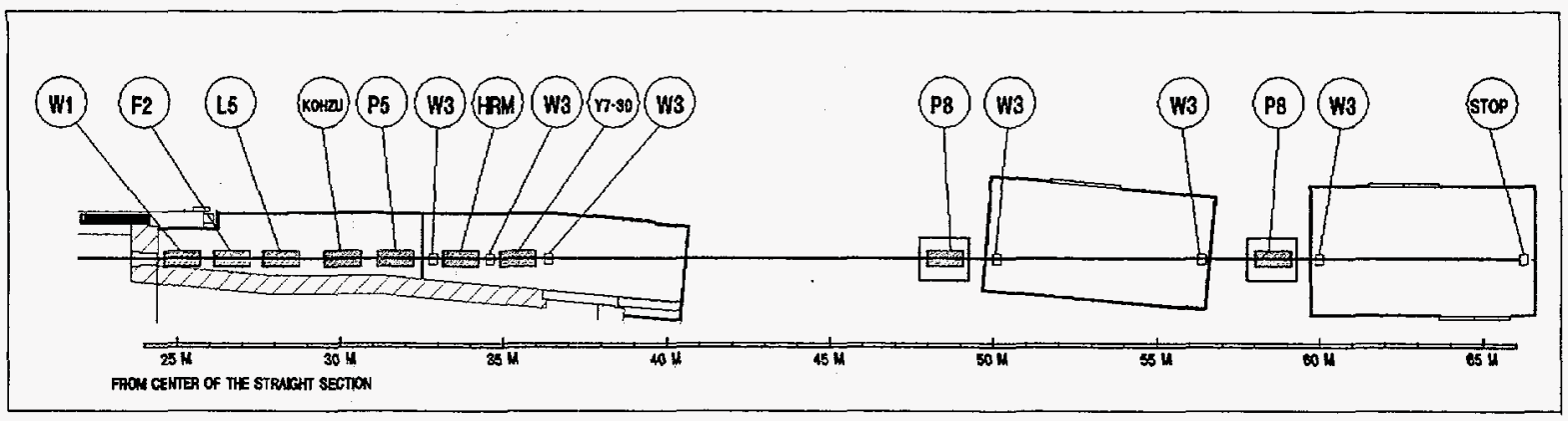

Fig. 4.62 Schematic layout of Sector 3-ID. W1 and W3: beryllium windows, F2: filter, L5: whitebeam slits, KOHZU: double-crystal high heat load monochromator, P5: white-beam stop, and monochromatic-beam shutter, HRM: high resolution monochromator (Kohzu), Y7-30: doublegroove mirror (Zeiss), P8: monochromatic-beam shutter

stages with $1 \mu \mathrm{m} / \mathrm{step}$ resolution. This system was custom built by Kohzu-Seiki Co., Japan (Model No: AAG-100). The flexibility of this system allows us to use both 4-bounce nested crystal geometry, as well as 2-bounce flat crystal geometry. The available set of monochromators are tabulated in Table 4.11.

The mirror is a novel design two-groove system on the same block with dual purposes: collimation and focusing. The cylindrical grooves can be bent pneumatically to a toroidal shape. The $80-\mathrm{cm}$-long zerodur mirror is coated with $\mathrm{Pd}$ to operate at a fixed angle of 2 mrad. The UHV-compatible system is contained with two Be windows on each end. The mirror and the bending mechanism are built by Zeiss, Germany.

Station 3-ID-C is dedicated to inelastic $\mathrm{x}$-ray scattering using backscattering crystal analyzers. There is a Huber diffractometer with a 2.6-m-long arm suitable to mount the analyzer. In addition, there are facilities for low temperature and an optical table.
Station 3-ID-D is dedicated to nuclear resonant scattering studies. It is equipped with an optical table, a 6-circle Huber goniometer, liquid He cryostat with reversible $7 \mathrm{~T}$ superconducting magnet, high-precision stages, and nanosecond timing electronics. Table 4.12 lists the characteristics and equipment associated with the 3-ID beamline.

\section{Current Status}

We commissioned the first two stations of this beamline in 1996. We have achieved a count rate of $1.2 \times 10^{13}$ photons $/ \mathrm{sec} / \mathrm{eV} / 100 \mathrm{~mA}$ at $14 \mathrm{keV}$ in the first harmonic of the undulator. The beam size is $0.8 \mathrm{~mm}$ by $2 \mathrm{~mm}$ at $35 \mathrm{~m}$ away from the source. Stations 3-ID $\mathrm{C}$ and 3-ID-D are scheduled to be commissioned in the April-June 1997 period. We also have plans to replace the liquid-gallium-cooled monochromator with either water-cooled diamond or liquid-nitrogen-cooled $\mathrm{Si}$, after testing the performance of both systems. 
Table 4.11 High Energy Resolution Monochromators Available at 3-ID

\begin{tabular}{|c|c|c|c|c|c|c|}
\hline & $\begin{array}{l}\text { Energy } \\
(\mathrm{keV})\end{array}$ & \multicolumn{2}{|c|}{ Bragg Reflections } & $\begin{array}{c}\text { Energy } \\
\text { Resolution } \\
\text { (meV) }\end{array}$ & $\begin{array}{c}\text { Angular } \\
\text { Acceptance } \\
\text { (urad) }\end{array}$ & Purpose \\
\hline 1. & 8.4 & Si (333) - (444) & channel-cut pair & 15 & 29 & Nested monochromator ${ }^{\mathrm{a}}$ for ${ }^{169} \mathrm{Tm}$ nuclear resonance \\
\hline 2. & 8.4 & $\mathrm{Si}(333)-(333)$ & channel-cut pair & $\mathrm{N} / \mathrm{A}$ & 44 & $\begin{array}{l}\text { Polarizer/Analyzer }{ }^{\mathrm{b}} \text { for }{ }^{169} \mathrm{Tm} \text { nuclear resonance. } \\
\text { Polarization rejection ratio: } 10^{-8}\end{array}$ \\
\hline 3. & 13.841 & Si $(440)-(844)$ & channel-cut pair & 5 & 20 & Nested monochromator for inelastic $\mathrm{x}$-ray scattering with Si (777) analyzer \\
\hline 4. & 14.413 & Si $(422)-(1064)$ & channel-cut pair & 5.5 & 22 & Nested monochromator for ${ }^{57} \mathrm{Fe}$ nuclear resonance \\
\hline 5. & 14.413 & Si $(975)-(975)$ & flat crystals & 0.9 & 8.4 & 2-bounce monochromator ${ }^{\mathrm{c}}$ for ${ }^{57} \mathrm{Fe}$ nuclear resonance \\
\hline 6. & 14.413 & Si $(975)-(975)$ & flat crystals & 0.66 & 12 & 2-bounce monochromator ${ }^{\mathrm{c}}$ for ${ }^{57} \mathrm{Fe}$ nuclear resonance \\
\hline 7. & 14.413 & $\mathrm{Si}(840)-(840)$ & channel cut pair & N/A & 10 & $\begin{array}{l}\text { Polarizer/Analyzer for }{ }^{57} \mathrm{Fe} \text { nuclear resonance. } \\
\text { Polarization rejection ratio: } 10^{-8}\end{array}$ \\
\hline 8. & 21.5 & $\mathrm{Si}(440),\left(\begin{array}{llll}15 & 11 & 3\end{array}\right)$ & flat crystals & 1 & 12 & 2-bounce monochromator for ${ }^{151} \mathrm{Eu}$ nuclear resonance \\
\hline 9. & 23.87 & $\mathrm{Si}(400)\left(\begin{array}{lll}12 & 12 & 12\end{array}\right)$ & flat crystals & 4.8 & 15 & 2-bounce monochromator for ${ }^{119} \mathrm{Sn}$ nuclear resonance \\
\hline 10. & 23.87 & $\mathrm{Si}(333)-(555)$ & channel-cut pair & 23 & 7 & Nested monochromator for ${ }^{119} \mathrm{Sn}$ nuclear resonance \\
\hline
\end{tabular}

a Nested monochromator refers to an arrangement of a higher order reflection channel-cut crystal nested into a lower order reflection channel cut crystal. The function of the lower order asymmetrically cut crystal is to increase the angular acceptance of the monochromator, while the higher order reflection crystals bring the energy bandpass down. The design and operational principles of nested monochromators are described in (1) T. Ishikawa, Rev. Sci. Inst. 63 (1992) 1015 (1992); (2) T. Toellner, SPIE proceedings, vol. 1740 (1992) 218. (1992); and (3) T. Mooney, Nucl. Instr. Meth. A347 (1994) 348.

b Polarizer/analyzer refers to an arrangement in which the channel cut crystals have Bragg angle close to $45^{\circ}$, satisfying the necessary condition for an effective polarization selection by providing a large angular acceptance, for one component of linear polarization while suppressing the other component and high reflectivity. By placing the polarizer and analyzer at $90^{\circ}$ with respect to each other, it is possible to measure any change in the state of polarization of the beam when it travels through an optically active medium. The details are described in T. Toellner, Appl. Phys. Lett. 67 (1995) 1993.

c 2-bounce flat crystals refer to a relatively new arrangement in which very high order reflection crystals with extreme asymmetric cuts are placed in dispersive geometry to minimize the energy bandpass while maintaining a reasonable angular acceptance. The details of these monochromators can be found in T. Toellner, Ph.D. Thesis, Northwestern University (1996), A. Chumakov, Nucl. Instr. Meth. A383 (1996) 642. 
Table 4.12 Sector 3, Insertion Device Beamline (3-ID)

\section{Responsible Scientists}

Ercan Alp, phone: (630) 252-4775, fax: (630) 252-9303, e-mail: eea@aps.anl.gov

Wolfgang Sturhahn, phone: (630) 252-0163, fax: (630) 252-0161, e-mail: sturhahn@aps.anl.gov

\section{Scientific Programs}

- High energy resolution $\left(10^{-5}<\mathrm{dE} / \mathrm{E}<10^{-13}\right) \mathrm{x}$-ray scattering in the $6-30 \mathrm{keV}$ range, for inelastic $\mathrm{x}$-ray and nuclear resonant scattering

- Development of crystal monochromators and analyzers

- Development of thin film optics

- Development of polarizer/analyzer optics

\section{Source Characteristics}

\begin{tabular}{|c|c|c|c|}
\hline \multicolumn{4}{|c|}{ Undulator } \\
\hline \multicolumn{2}{|l|}{ Source size } & \multicolumn{2}{|c|}{$0.3 \times 0.029 \mathrm{~mm}^{2}$ (horiz. $\times$ vert.) FWHM } \\
\hline \multirow{2}{*}{\multicolumn{2}{|c|}{ Source divergence }} & \multicolumn{2}{|c|}{$0.024 \times 0.003 \mathrm{mrad}^{2}$ (horiz. $\times$ vert.) FWHM } \\
\hline & & \multicolumn{2}{|c|}{$2.5 \mathrm{~m}$} \\
\hline \multicolumn{2}{|l|}{ Periods } & \multicolumn{2}{|l|}{$2.7 \mathrm{~cm}$ and $3.3 \mathrm{~cm}$ (currently not installed) } \\
\hline \multicolumn{2}{|l|}{ Energy range } & \multicolumn{2}{|l|}{$3 \mathrm{keV}$ and above } \\
\hline \multicolumn{4}{|l|}{ Optical Components } \\
\hline Component & $\begin{array}{l}\text { Distance from } \\
\text { Source }(\mathrm{m})\end{array}$ & Description & $\begin{array}{c}\text { Date } \\
\text { Available }\end{array}$ \\
\hline Horiz. and vert. slits & 28 & $25 \mu \mathrm{m}$ reproducibility & $01 / 1996$ \\
\hline Double crystal mono. & 29.82 & Kohzu, Si (111) & $01 / 1996$ \\
\hline Double crystal mono. & 33 & Kohzu, high resolution ( $12.5 \mathrm{nrad} / \mathrm{step}$ stages) & $01 / 1996$ \\
\hline Mirror & 34.5 & Zeiss, $80 \mathrm{~cm}$, double groove (collimate and focus) & $07 / 1997$ \\
\hline \multicolumn{4}{|c|}{$\begin{array}{l}\text { Beamline Configurations } \\
\text { 3-ID-A: white beam } \\
\text { 3-ID-B: monochromatic beam } \\
\text { 3-ID-C: monochromatic beam } \\
\text { 3-ID-D: monochromatic beam }\end{array}$} \\
\hline \multicolumn{2}{|c|}{ 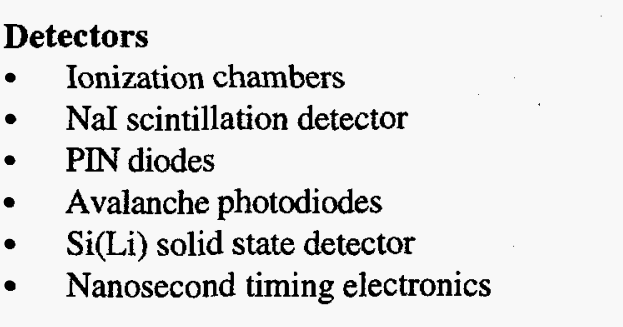 } & \multicolumn{2}{|l|}{$\begin{array}{l}\text { Beamline Control } \\
\text { - EPICS }\end{array}$} \\
\hline \multicolumn{2}{|c|}{$\begin{array}{l}\text { Ancillary Equipment } \\
\text { - Optical tables with } 6 \text { degrees of freedom } \\
\text { - } \quad \text { Precision scanning stages } \\
\text { - } \text {-Testats } \\
\text { Huber golenoid cryostat (Oxford) } \\
\text { Huber 6-circle diffractometer } \\
\quad \text { Euler stages }\end{array}$} & \multicolumn{2}{|l|}{$\begin{array}{l}\text { Beamline Installation } \\
\text { The beamline is to be completed } 06 / 1997 \text {. }\end{array}$} \\
\hline
\end{tabular}




\section{High resolution optics}

Our highest priority was to improve the energy resolution of the monochromators. Along these lines we have tested three classes of monochromators:

- Nested monochromators (4-bounce)

- Flat crystals (2-bounce)

- Polarizer/analyzers as monochromators

We have built two sets of nested monochromators to work at $14.4 \mathrm{keV}$ for nuclear resonant scattering studies and at $13.8 \mathrm{keV}$ for inelastic $\mathrm{x}$-ray scattering coupled to a Si (777) backscattering analyzer. The energy resolution is $5 \mathrm{meV}$, and the flux achieved is $2 \times 10^{9} \mathrm{~Hz}$ in this bandpass.

In order to reduce the energy bandpass further, we have prepared high order Bragg reflection crystals with asymmetry angles close to the Bragg angle. The first crystal, for example, is a Si (975) with a Bragg angle of 80.4 degrees, and it is asymmetrically cut with $b=1 / 22.3$, and the second crystal with $b=10.4$. This crystal pair has an angular acceptance of $8.5 \mu \mathrm{rad}$. When the asymmetry angles are changed such that $b=1 / 38$ for the first crystal and $b=22.3$ for the second crystal, the energy bandpass is further reduced to $0.66 \mathrm{meV}$, while the angular acceptance is increased to $11 \mu \mathrm{rad}$. The measured flux is $0.4 \mathrm{GHz}$ at $0.9 \mathrm{meV}\left(\Delta \mathrm{E} / \mathrm{E}=5.7 \times 10^{-8}\right)$, and $0.2 \mathrm{GHz}$ at $0.66 \mathrm{meV}$ resolution $(\Delta \mathrm{E} / \mathrm{E}=4.5 \times$ $\left.10^{-8}\right)$. This represents $4 \%$ efficiency, while the theoretical limit is about $10 \%$. The energy resolution, measured using coherent nuclear forward scattering, is shown in Fig. 4.63b, along with phonon density of states measured via inelastic nuclear resonant scattering,
Fig. 4.63a. These monochromators are the first instruments suitable to measure lattice excitations with sub-meV resolution using $\mathrm{x}$-rays.

Bragg reflections near $45^{\circ}$ act as $\mathrm{x}$-ray polarizers. With the availability of undulator sources, the same type of crystals can be used as analyzers. Such a pair has been used to generate a monochromatic beam with an energy bandpass of $0.8 \mu \mathrm{eV}$ with about $10^{4} \mathrm{~Hz}$ flux. The angular acceptance of Si (8 40 ) reflection is $10.2 \mu \mathrm{rad}$ when the asymmetry angle is $-43^{\circ}$ for a Bragg angle of $45.1^{\circ}$. This should be compared to the $15 \times$ $52 \mu \mathrm{rad}$ vertical and horizontal divergence of undulator radiation. This discrepancy may be remedied when the collimating branch of the mirror is operational. The polarization switching was provided with an iron thin film in grazing-incidence geometry, with a magnetic field applied along the incident beam.

\section{Vibrational dynamics of thin films}

The high energy resolution (meV) inelastic neutron and $\mathrm{x}$-ray scattering studies have been limited to bulk materials. This is partly due to the high penetration of neutrons, as well as the lower brightness of neutron sources. In the case of conventional inelastic $\mathrm{x}$-ray scattering, the scattering from the sample has to originate from a small spot size to lend itself to energy analysis with meV resolution, thus precluding grazing-incidence geometry where the beam is spread over a large area. These obstacles have been overcome in case of inelastic nuclear resonant scattering under grazing-incidence geometry. By enhancing the electric field amplitude in the region where the thin film is deposited, it is possible to increase the signal 


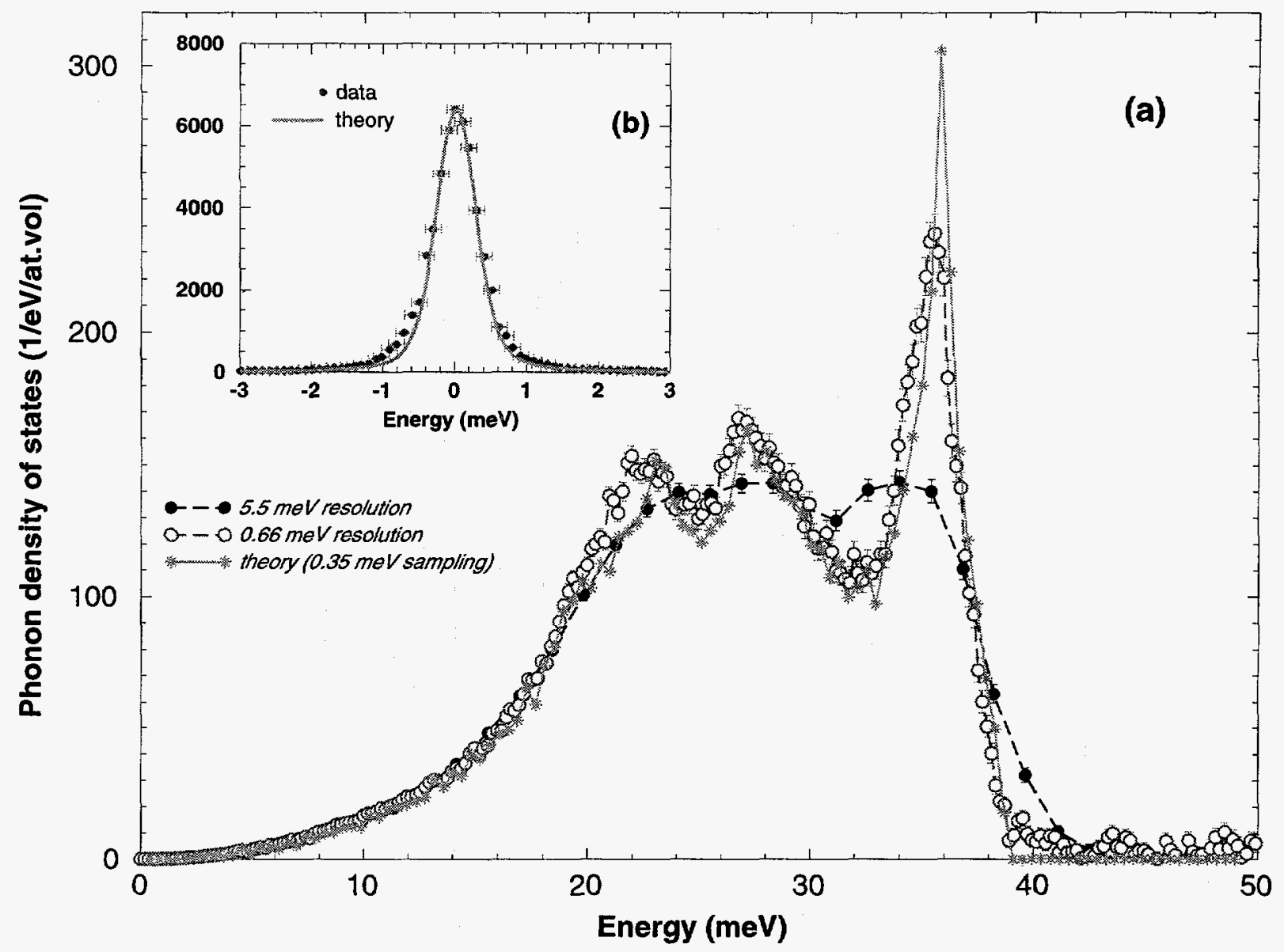

Fig. 4.63 (a) The photon density of states of iron measured by inelastic nuclear resonant scattering. The black filled circles were measured with the 5.5-meV nested monochromator, while the empty circles were measured with 0.66-meV-resolution, 2-bounce flat crystals. The theory curve was calculated from experimentally determined force constants via fitting the coherent neutron scattering data. (b) The inset shows the resolution function of the $0.66 \mathrm{meV}$ monochromator as measured with coherent nuclear forward scattering. The theoretical calculation includes the brilliance function of the incident beam coupled to the dynamical diffraction equations.

similar to the bulk values. This increase in the inelastic fluorescence yield is shown in the Fig. 4.64 at $4 \mathrm{mrad}$. The incident beam energy was set at $20 \mathrm{meV}$ above the nuclear resonance energy. This technique is applied to $\mathrm{Fe}_{1-\mathrm{x}} \mathrm{Tb}_{\mathrm{x}}$ amorphous thin films with a thickness of $175 \AA$. A series of samples are measured as a function of $x$, and in addition to phonon density of states (Fig. 4.65a), force constants are derived from the third moment of the phonon spectrum (Fig. 4.65b).

\section{Phonon density of states measurements of iron and iron oxides}

The direct measurement of phonon density of states with sub-meV resolution using $\mathrm{x}$-rays has been elusive, because neither monochromatization nor analysis has been trivial. By using nuclear resonance as an analyzer, the energy gain or loss of a photon during 


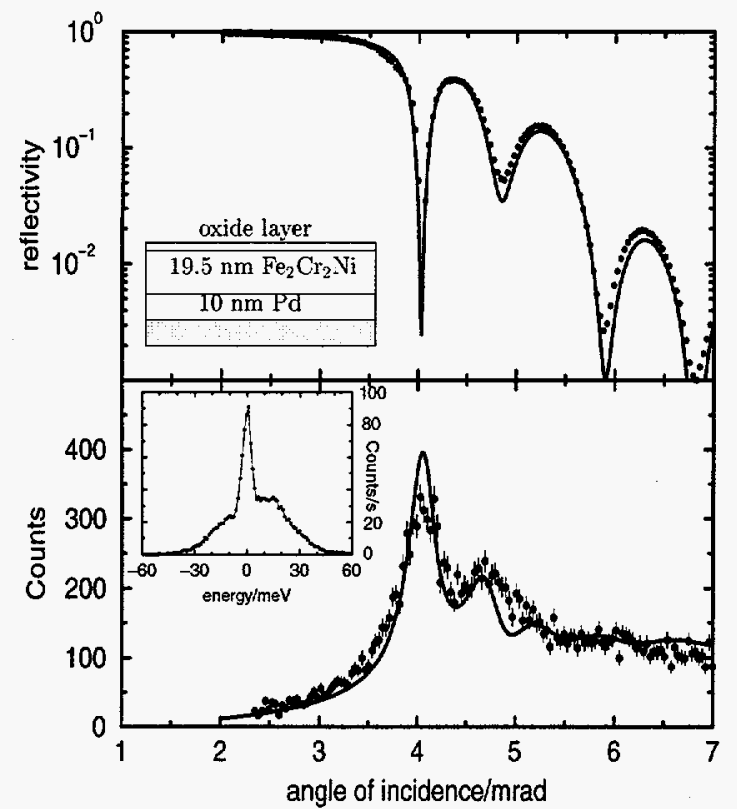

Fig. 4.64 The principle of phonon density of states measurements of thin films: (Top) The electronic reflectivity of $\mathrm{Fe}_{2} \mathrm{Cr}_{2} \mathrm{Ni}$ film as a function of incidence angle. The sharp drop at 4 mrad corresponds to a cancellation of the total external reflected beam at the grazing incidence anti-reflection point. (Bottom) The delayed fluorescence yield as a result of nuclear decay, whose excitation is caused by phonon creation at $+20 \mathrm{meV}$ above the nuclear resonance energy. The enhanced yield at 4 mrad is caused by the increase in electric field amplitude in the film due to total external reflection from the Pd layer, and it coincides with the drop in reflectivity at the grazing incidence anti-reflection point. (Inset) The inelastic nuclear scattering data obtained as a function of incidence energy. A 5.5-meVresolution nested monochromator was used in this experiment. The negative energies correspond to phonon annihilation, while the positive energies correspond to phonon creation.
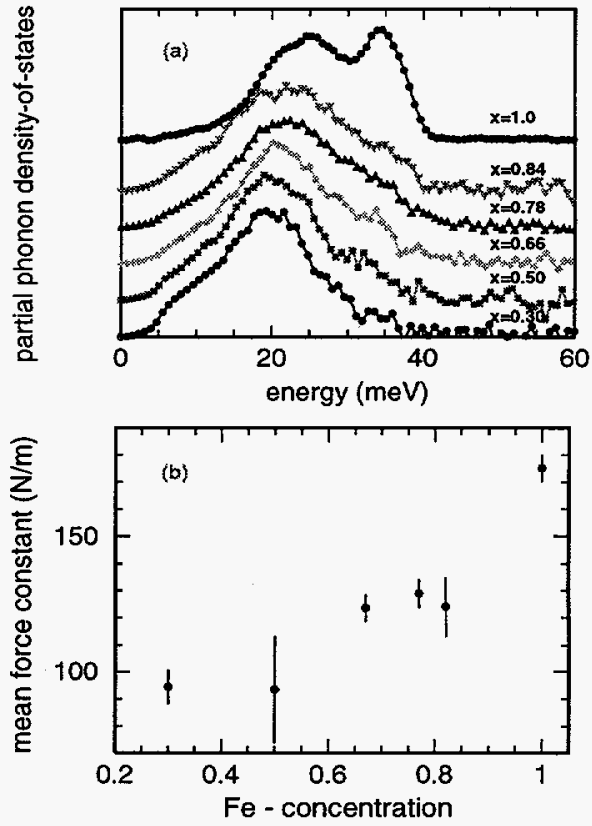

Fig. 4.65 (a) Phonon density of states of a Fe ${ }_{1-x} \mathrm{~Tb}_{x}$ system, as a function of $x$. (b) The force constants derived from the third moment of phonon creation-annihilation spectra as a function of $x$. Such measurements with thin films are facilitated by employing grazingincidence geometry and nuclear resonance as an analyzer to measure lattice excitations.

interaction with lattice excitations can be measured, as shown in Fig. 4.66a. It is possible to extract phonon density of states (Fig. 4.66b). Using the $0.66 \mathrm{meV}$ monochromator, the phonon density of states of iron metal and various iron oxides has been measured.

\section{Test of backscattering analyzers}

The energy resolution of several backscattering analyzers has been measured. We have reached an energy resolution of $9 \mathrm{meV}$ 

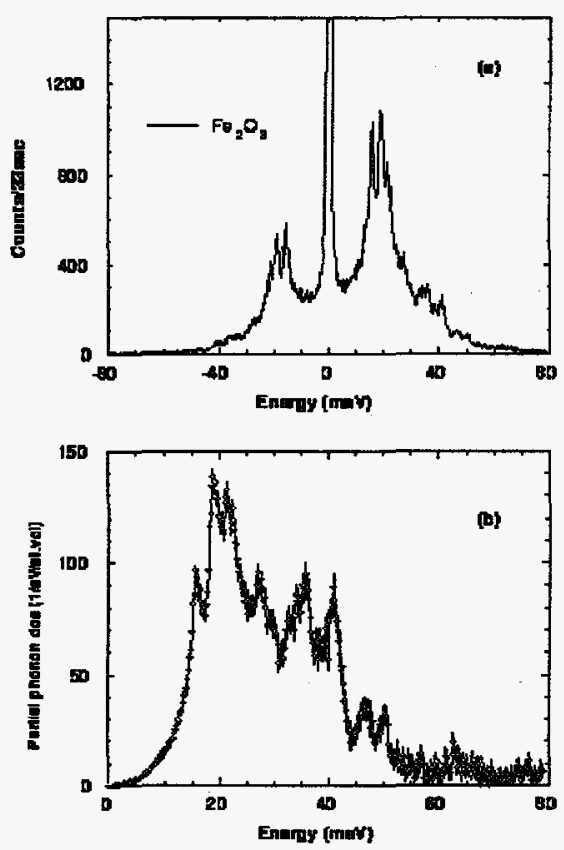

Fig. 4.66 (a) The inelastic nuclear resonant scattering yield as a function of incidence energy for a hematite sample, using 0.66-meV-resolution, two-bounce flat crystals as a monochromator. (b) The phonon density of states derived from the data in (a) indicates many sharp singularities, corresponding to low dispersion points in optical, and perhaps, acoustical phonons.

at $13.842 \mathrm{keV}$. This analyzer, coupled with an in-line nested monochromator with 5-meV bandpass, was used to study a particular optical branch in diamond to verify a maximum in the phonon dispersion curve (see Fig. 4.67). This set of measurements has demonstrated the advantage of scanning monochromator by changing the angle, rather than the temperature.

\subsubsection{New R\&D Initiatives}

The primary new initiative for SRI-CAT is the development of an ID beamline in Sector 4 .

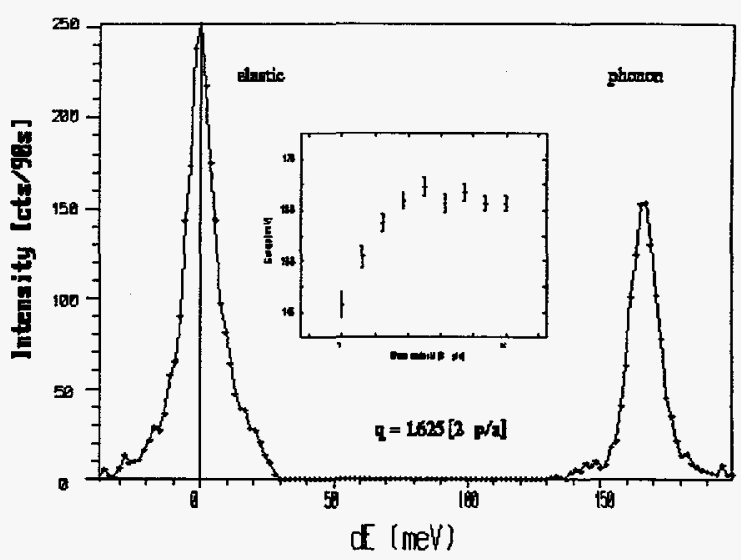

Fig. 4.67 Observation of the longitudinal optical phonon along the $\langle 100\rangle$ direction in diamond. This has been achieved by using a nested 4-bounce monochromator with 5-meV resolution and a Si (777) backscattering analyzer, giving a total resolution of $12 \mathrm{meV}$. The inset shows the dispersion of this mode as a function of momentum transfer.

The unique feature of this beamline will be the availability of $\mathrm{x}$-rays with variable polarization from $0.5 \mathrm{keV}$ to $100 \mathrm{keV}$ and higher. This will be accomplished in the low energy range $(0.5 \mathrm{keV}$ to $5 \mathrm{keV})$ with a helical undulator and with crystal optics and with APS undulator A for energies above $5 \mathrm{keV}$. These unique tunable polarization and energy capabilities, located on one beamline, will provide scientists with the ability to cover all important absorption edges from $\mathrm{M}$ to $\mathrm{K}$ for magnetic spectroscopy experiments and resonant magnetic scattering.

\subsection{References}

Amir, A., K. Vafai and T.M. Kuzay, Numerical Heat Transfer, Part A, 27 (1995) 651-664 
Bathow, G., E. Freytag, and K. Tesch, Nucl. Phys. B2 (1967) 669

Barraza, J., D. Shu and T.M. Kuzay, Nucl. Instrum. and Meth. A 347 (1994) 591-597

Barraza, J., D. Shu, and T.M. Kuzay, Rev. Sci. Instrum. 66 (1995) 1630-1632

Basdogan, I., T. Royston, A. Shabana, D. Shu, T. Kuzay, SPIE Proc. Vol. 2865 (1996) 1-14

Bilderback, D.H., Nucl. Instrum. Meth. 246 (1986) 434

Blasdell, R.C., L.A. Assoufid, and D.M. Mills, Argonne National Laboratory Report ANL/APS/TB-24 (1995)

Burkel, L., R. Dejus, J. Maines, J. O'Brian, J. Pflüger, and I. Vasserman, Argonne National Laboratory Report ANL/APS/TB-12 (1993)

Cai, Z., R.J. Dejus, P. Den Hartog, Y. Feng, E. Gluskin, D. Haeffner, P. Illinski, B. Lai, D. Legnini, E.R. Moog, S. Shastri, E. Trakhtenberg, I. Vasserman, and W. Yun, Rev. Sci. Instrum. 67 (9) (1996a) CD-ROM

Cai, Z., B. Lai, W. Yun, E. Gluskin, D. Legnini, P. Illinski, and G. Srajer, Rev. Sci. Instrum. 67 (9) (1996b) CD ROM

Chang, J., D. Shu, H.L. Nian, T.M. Kuzay and P.K. Job, Rev. Sci. Instrum. 66 (1995) $1648-50$

Chavanne, J., P. Elleaume, and P. Van Vaerenbergh, ESRF Machine Technical Note 1-1996/ID (1996)
Colomp, P., and E. Bräuer, ESRF Report 143/93/EB (1993)

Dejus, R.J., and M. Sanchez del Rio, Rev. Sci. Instrum. 67 (9) (1996) CD ROM

Dejus, R.J., and A. Luccio, Nucl. Instrum. and Meth. 347 (1994) 61-66

Den Hertog, P., J. Grimmer, T. Klippert, E. Trakhtenberg, and S. Xu, Rev. Sci. Instrum. 67(9) (1996) CD ROM

Ehrfeld, W., and D. Münchmeyer, Nucl. Instrum. Meth. A303 (1991) 523

Ehrfeld, W., and H. Lehr, Synch. Rad. News 7 (5) (1994) 9

Feng, Y.P., S.K. Sinha, E.E. Fullerton, G. Grubel, D. Abernathy, D.P. Siddons, and J.B. Hastings, Appl. Phys. Lett. 67 (1995) 3647

Frachon, D., I. Vasserman, P.M. Ivanov, E.A. Medvedko, E. Gluskin, and N.A. Vinokurov, Argonne National Laboratory Report ANL/APS/TB-22 (1995)

Khounsary, A.M., and W. Yun, Rev. Sci. Instrum. 67 (9) (1996) CD ROM

Khounsary, A.M., W. Yun, E. Trakhtenberg, S. Xu, L. Assoufid, and W. K. Lee, SPIE Proc. 2855 (1996) 232-239

Knapp, G.S., M.A. Beno, C.S. Rogers, C.L. Wiley, and P.L. Cowan, Rev. Sci. Instrum. 65 (9) (1994) 2792-2797 
Knapp, G.S., C.S. Rogers, M.A. Beno, C.L. Wiley, G. Jennings, and P.L. Cowan, Rev. Sci. Instrum. 66 (2) (1995) 2138-2140

Kuzay, T.M, J.T. Collins, A.M. Khounsary, and G. Morales, ASME JJSME 3rd Joint Heat Conference, Book No. I0309E-1991 (1991) 451-459

Kuzay, T. M., Rev. Sci. Instrum. 63 (1992) 468

Kuzay, T.M., Argonne National Laboratory Report ANL/APS/TB-5 (1993)

Kuzay, T. M., Nucl. Instrum. Meth. Phys. Res. A 347 (1994) 644-650

Kuzay, T.M., H.L.T. Nian, Z. Wang, and D. Shu, Rev. Sci. Instrum. 67 (9) (1996) CD ROM

Lai, B., D.C. Mancini, W. Yun, and E. Gluskin, SPIE Proc. 2880 (1996) 171-176

Lee, W.K., and D. Mills, Argonne National Laboratory Report ANL/APS/TB-4 (1993)

Liu, C., R. Nielsen, T.L. Kruy, D. Shu, and T.M. Kuzay, Rev. Sci. Instrum. 66 (1995) 2703-2707

Liu, C., D. Ryding, R.W. Nielsen, T.L. Kruy, and T.M. Kuzay, Rev. Sci. Instrum. 67 (1996) CD ROM

McNulty, I., A.M. Khounsary, Y.P. Feng, Y. Qian, J. Barraza, C. Benson, and D. Shu, Rev. Sci. Instrum. 67 (9) (1996) CD-ROM
Mulhaupt, G., Rev. Sci. Instrum. 66 (1995) 2000

Okuda, S., K. Ohashi, and N. Kobayashi, Nucl. Instrum. Meth. 94 (1994) 227

Rehn, V., SPIE Proc. 582 (1985) 238-250

Rogers, C.S., D.M. Mills, W.-K. Lee, G.S. Knapp, J. Holmberg, A. Freund, M. Wulff, M. Rossat, M. Hanfland, and H. Yamaoka, Rev. Sci. Instrum. 66 (6) (1995) 3494-3499

Rogers, C.S., D.M. Mills, L. Assoufid, and T. Graber, Rev. Sci. Instrum. 67 (9) (1996a) CD ROM

Rogers, C.S., D.M. Mills, W.-K. Lee, P.B. Fernandez, and T. Graber, SPIE Proc. 2855 (1996b) 170-179

Rogers, C.S., D.M. Mills, P.B. Fernandez, G.S. Knapp, M. Wulff, M. Hanfland, M. Rossat, A. Freund, G. Marot, J. Holmberg, and H. Yamaoka, Rev. Sci. Instrum. 67 (9), (1996c) CD ROM

Samal, P.K., and A.V. Nadkarni, Modern Development in Powder Metallurgy 16 (Metal Powder Industries Federation American Powder Metallurgy Institute, 1984)

Samal, P.K., The Metal Science of Joining, (The Minerals, Metals \& Materials Society, 1992)

Shu, D., J. Barraza, T. Sanchez, R.W. Nielsen, J.T. Collins, and T.M. Kuzay, Nucl. Instrum. Meth. Phys. Res. A 319 (1992a) 63-70 
Shu, D., B. Rodricks, J. Barraza, T. Sanchez and T.M. Kuzay, Nucl. Instrum Meth. in Phys. Res. A 319 (1992b) 56-62

Shu, D., T. Nian, Z. Wang, J.T. Collins, D.G. Ryding, and T.M. Kuzay, SPIE Vol. 1739 (1992c) 218-229

Shu, D., and T.M. Kuzay, Nucl. Instrum. Meth. Physics Research A 347 (1994) 584-90

Shu, D., J.T. Collins, J. Barraza, and T.M. Kuzay, Nucl. Instrum. Meth. in Phys. Res. A 347 (1994) 577-580

Shu, D., J. Barraza, C. Brite, T. Sanchez, and V. Tcheskidov, Rev. Sci. Instrum. 66 (1995) 1795-1797

Shu, D., and T.M. Kuzay, Rev. Sci. Instrum. 67 (9) (1996) CD ROM

Shu, D., J. Barraza, W. Yun, K. Randall, B. Lai, I. McNulty, J. Xu, A.M. Khounsary, E. Gluskin, and T.M. Kuzay, Rev. Sci. Instrum. 67 (9) (1996) CD-ROM

Smither, R.K., and P. Fernandez, Nucl. Instrum. and Meth. 347 (1994) 313-319

Sözen, M., and T.M. Kuzay, Int. J. Heat and Fluid Flow, 17 (1996) 124-129

Tonnessen, T.W., S. Fisher, F. Antony, D. Lunt, A. Khounsary, K. Randall, E. Gluskin, and W. Yun, SPIE Proc. 1997 (1993) 340-353

Tonneson, T.W., A. Khounsary, W. Yun, and D. Shu, SPIE Proc. 2885 (1996) 187-198
Trakhtenberg, E., E. Gluskin, P. Den Hartog, T. Klippert, G. Wiemerslage, and S. Xu, Proc. of the 1995 Particle Accelerator Conf. (1996) 2072-2074

Vasserman, I., Argonne National Laboratory Report LS-253 (1996)

Walker, R. P., Nucl. Instrum. Meth. 335 (1993) 328

Wang, Z., T. Nian, D.G. Ryding, T.M. Kuzay, Nucl. Instrum. Meth. Phys. Res. A 347 (1994) 651-656

Warwick, T., et al., Rev. Sci. Instrum. 66 (1995)

Yun, W., A. Khounsary, B. Lai, and E. Gluskin, Argonne National Laboratory Report ANL/APS/TB-2 (1992)

Yun, W., A. Khounsary, B. Lai, K. Randall, I. McNulty, E. Gluskin, and D. Shu, Rev. Sci. Instrum. 67 (9) (1996a) CD-ROM

Yun, W., B. Lai, D. Shu, A. Khounsary, Z. Cai, J. Barraza, and D. Legnini, Rev. Sci. Instrum. 67 (9) (1996b) CD-ROM.

\section{PATENTS}

Kuzay, T., Process of Making Cryogenically Cooled High Thermal Performance Crystal Optics, U.S. Patent No. 5,123,982, granted June 1992

Kuzay, T., and D. Shu, Photon Beam Position Monitor, U.S. Patent No. 5,387,795, granted February 1995 
Shu, D., and T. Kuzay, Integral Window/ Photon Beam Position Monitor and Beam Flux Detectors for X-Ray Beams, U.S. Patent No. 5,404,014, granted April 1995
Shu, D., and J. Barraza, Low-Profile, HighLoad, Vertical Rolling Positioning Stage, U.S. Patent No. 5,526,903, granted June 1996 

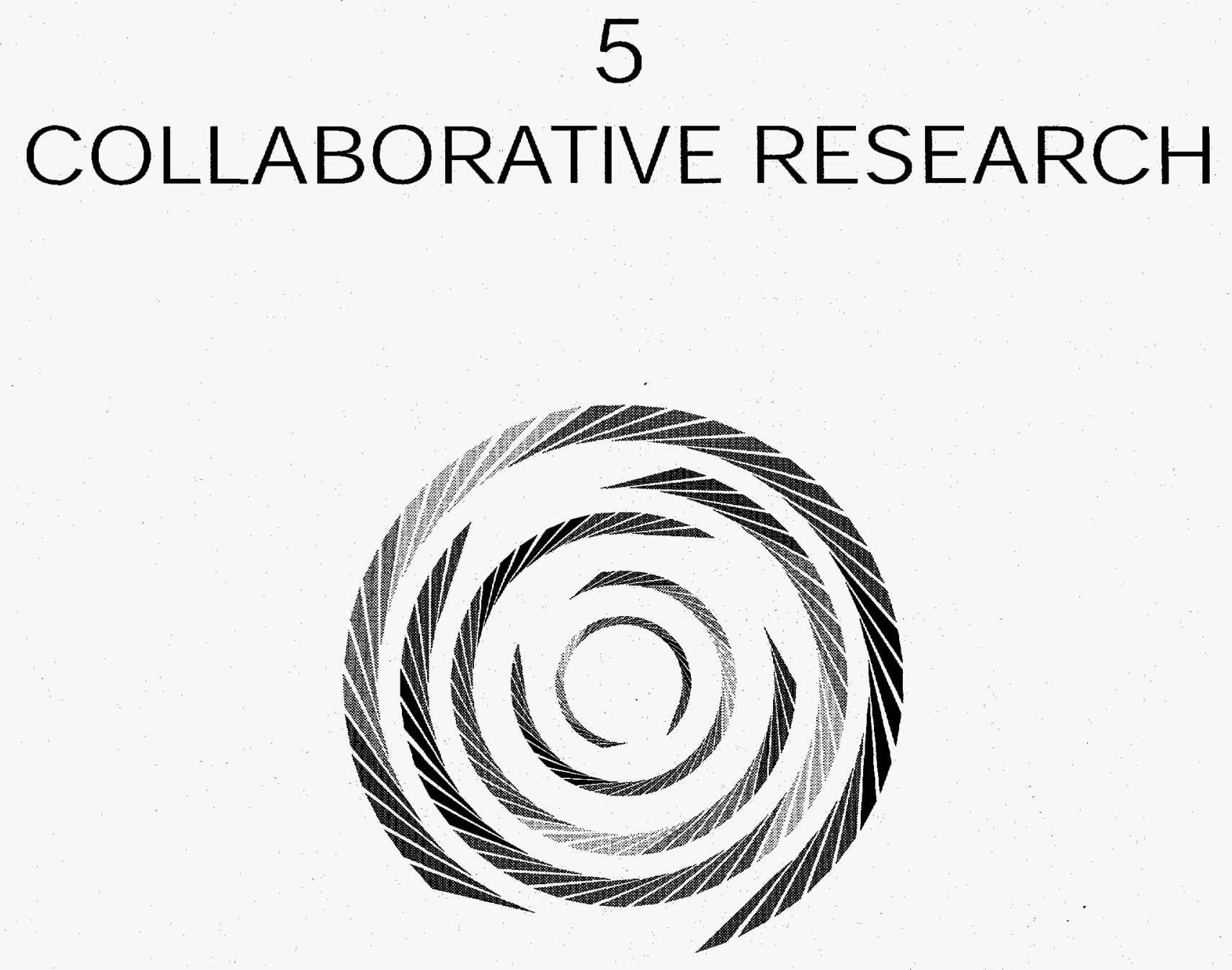


\subsection{Introduction}

The XFD staff constantly looks for opportunities for collaboration with others in the synchrotron radiation community. This is consistent with the mission of XFD, and rewards from such interactions have been excellent both for XFD staff and the users.

\subsection{Collaborative Research Programs}

To optimize the use of the APS for its user community, the XFD staff will conduct collaborative R\&D with APS users. For example, in 1991, XFD established a Collaborative Research Program (CRP) with potential users of the APS. In this program, a joint proposal is made by XFD staff members and interested APS users to perform R\&D on a topic of general interest to APS users. The projects to be pursued for these collaborations are selected by means of a competitive proposal process. The $\mathrm{R} \& \mathrm{D}$ is conducted jointly, with personnel and resources provided by the proposing institution or CAT and the XFD.

Such CRPs were very popular when the process was first introduced. The success stories from these collaborative interactions are many. A couple examples are given here. One of the programs proposed by Sol Gruner of Princeton involved development of pixel array detectors. This program has now matured, and Gruner was awarded \$1.38 M by DOE to continue the extension of the CRP. Similarly, Mihai Popovici of the University of Missouri carried out a CRP with XFD staff on the development of bent crystals for focusing monochromators. Popovici has now proposed to extend this work with a DOE grant of $\$ 0.45 \mathrm{M}$.
During the past three years, APS users have been very busy in the construction and commissioning of their beamlines at the APS and have not proposed any new CRPs. New calls for CRPs will be issued during the summer of 1997.

\subsection{Special Grants for New Collaborative Research}

The staff in XFD has also been very successful in obtaining research support for proposals jointly submitted with the APS users. Two recent awards are outlined below.

1. A DOE Grand Challenge Grant given to XFD staff for joint work with researchers from the Mathematical and Computer Science Division of ANL, CARS, and SBC. The subject of this work is: Supercomputer Solution of Massive Crystallographic and Microtomographic Structural Problems.

The work proposed supports the XFD mission and is also relevant to DOE. The tomographic reconstruction supports work of the DOE Divisions of Engineering and Geosciences and Material Science. The work on direct phase solution of crystallographic structure is of primary interest to the Office of Health and Environmental Research.

Work on computational infrastructure will produce new techniques for coupling supercomputers with advanced instruments over high speed local and wide area networks, for remote visualization and monitoring results, for large-scale optimization, and for managing and visualizing very 
large data sets. These capabilities are an important step in the use of computer power and networks and should have a broad impact on all APS users.

2. An award given to the staff of the Oriental Institute at The University of Chicago and XFD staff by the Joint Research Support Program of The University Chicago and ANL. The work will lead to the first applications of synchrotron radiation in the field of archaeometallurgy. This collaboration will facilitate interactions between $\mathrm{x}$-ray experimentalists from XFD and archaeologists from the Oriental Institute, The University of Chicago, in a way that will accelerate the rate of transfer of modern $\mathrm{x}$-ray techniques to a new field.

The effort is also expected to facilitate the training of graduate students from archaeology in modern $\mathrm{x}$-ray techniques.

\subsection{Collaborative Projects with Other Synchrotron Radiation Facilities}

The XFD staff is often requested to support work in the design and fabrication of major instrumentation for various other synchrotron radiation facilities. Such work is performed either in collaboration or through a full cost recovery plan. Some examples follow:

a. Construction and commissioning of an elliptical multipole wiggler at the NSLS X-ray Ring was performed in collaboration with the Brookhaven
National Laboratory staff. This source of circularly polarized radiation with switchable helicity is now used at the NSLS by the users.

b. Tuning of the APS-CHESS prototype undulator to meet the stringent magnetic tolerances of a microwave FEL being developed at NSLS.

c. Design and construction of undulator vacuum chambers for BESSY II. The cost of this work was fully recovered from BESSY.

d. Design and construction of prototypes of photon masks and photon shutters for four undulator beamlines at SPring-8. This continuing work is fully paid for by SPring-8.

e. There have been four joint APSESRF-SPring-8 workshops during the past four years. These workshops are primarily for the facility staff of all three institutions to exchange their experience both in the accelerator and experimental areas. These workshops have had great value in sharing information and performing joint experiments. For example, in XFD, much of the early work on high heat load optics was carried out jointly at ESRF beamlines by scientists from the three facilities. Design and construction of small-aperture undulator vacuum chambers and $\mathrm{x}$-ray beam position monitors for the undulator beamline front end were also performed at the ESRF beamline. This joint collaboration between APS, ESRF and SPring-8 is expected to continue in future years. 
The success of the above projects is a true testimonial to the world-wide recognition of XFD staff. (See Appendix 9 for a list of invited talks.) 
5 COLLABORATIVE RESEARCH 


\section{6 \\ LONG-TERM STRATEGIC PLANS FOR XFD}

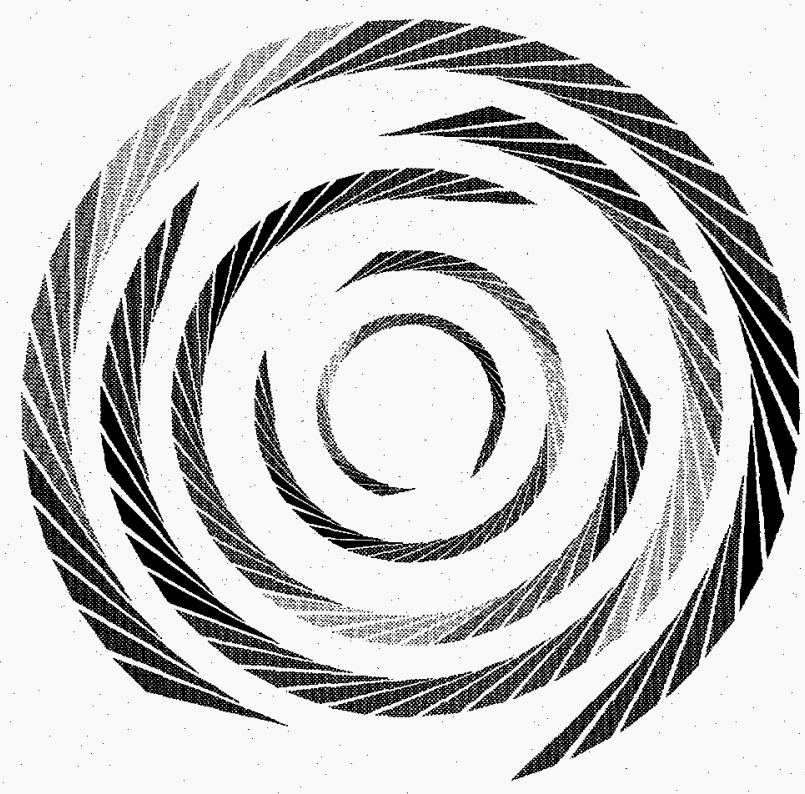




\subsection{Introduction}

The following are important areas to be considered in any future plans of the XFD:

- Adequate support is required for improvement of the operating instruments in order to provide the highest reliability for user operations at the APS facilities. Some of these needs are met with the limited funds made available by the DOE under the Accelerator Improvement Program (AIM).

- $\quad \mathrm{R} \& \mathrm{D}$ is necessary at this time to fully utilize the potential of the APS. This includes operating the APS in a "topoff" mode in which stored beam current is kept at a nearly constant value. In addition, the APS will be operated in the future at $300 \mathrm{~mA}$ of stored particle beam. Handling the heat load of the radiation from such a beam with possible 5-m-long undulators requires $R \& D$ on some components of the beamline. This work needs to begin immediately in order to fully exploit the high brilliance of which the APS is capable.

- Planning for the implementation of beamlines in the remaining 14 sectors of the storage ring is necessary. This is consistent with DOE's highest priority to fully and effectively utilize currently operating facilities. The first 20 sectors of the APS are being utilized to support the general scientific goals of the already established CATs. New collaborative teams are likely to be formed in the coming years. XFD is constantly looking for opportunities to attract new communities interested in using the APS.

- The research and development objectives of the present CATs are broad, and thus beamline instruments were designed to cater to the general community. While this approach has been essential for the support of the U.S. synchrotron community at large, it has precluded exploration of dedicated scientific areas for which long-term development of specialized instruments is required. Such dedicated beamlines built around focused user groups will be proposed to utilize some of the remaining 14 sectors. These proposals will be developed such that the special purpose beamlines will be jointly built by XFD and the user groups at a rate of 1-2 beamlines per year starting in 1999.

- There is world-wide interest in a so-called fourth-generation synchrotron radiation facility, which will be an $\mathrm{x}$-ray free-electron laser (FEL) based on a linac using the "self-amplification of stimulated emission" (SASE) concept. The APS linac, with its $450-600 \mathrm{MeV}$ capability, is suitable for testing the SASE concept in the 500-1000 A range. Superior expertise and experience in the field of undulator development, so essential for any $\mathrm{x}$-ray FEL effort, is singularly available in XFD. This would be another initiative requiring major $R \& D$ effort.

Strategic planning has been performed by XFD to develop new initiatives to meet the future APS goals indicated above. Considerable $R \& D$ is required in order to prepare these initiatives, as is continued dialogue with the 
user community. Support for all such R\&D activities is currently not provided through the normal DOE funding process. Hence support is derived for many activities from the LDRD funds distributed by the ANL Director through a laboratory-wide competitive process.

XFD also constantly seeks other sources of funds to support $R \& D$, performed in collaboration with the APS users, that can either lead to new capabilities for the users or support possible new initiatives. These efforts were addressed in Chapter 5.

\subsection{Accelerator Improvement Program Plans}

The following AIM activities have been planned for 1997 and 1998 in order to improve the quality of XFD operations in support of APS users:

1. Extract storage-ring, ID, and beamline front-end operational parameters and provide the data to APS users in a usable format in real time.

2. Install higher accuracy and more reliable linear encoders on all 18 undulators to provide real-time gap information to user beamlines.

3. Interface critical PSS and EPS parameters with the APS control system.

4. Fabricate and install smaller-aperture front-end masks.

5. Develop interface and software to provide continuous monitoring of the ID front-end vacuum.
6. Install residual gas analyzers for ID vacuum chambers.

7. Expand the PSS-EPICS interface for both BM and ID beamlines.

8. Upgrade ID stepping-motor power supplies and controllers.

9. Install UHV diagnostics instrumentation and component test enclosures.

10. Add a PSS electronic validation system.

11. Replace vacuum hardware in $\mathrm{D}$ chambers.

12. Install DSP units for the XBPMs in the ID front ends.

13. Replace front-end critical components.

14. Add oven and magnetization systems to treat ID magnets.

\subsection{Enhancement of APS Performance}

The following three areas reflect future enhancements of APS performance, which will require upgrades of certain components maintained by XFD:

- Providing 5.0-m-long undulators to specific CATs

- Implementing "top-off" mode to keep stored beam current nearly constant 
- Operating the storage ring with stored current of $300 \mathrm{~mA}$

The R\&D in support of these activities has been planned for 1998-99. Operation of the storage ring with the above enhancements requires additional capital funds to install upgraded components. For example, all the undulators currently provided to users are $2.5 \mathrm{~m}$ long, and longer devices will require additional funds for construction and installation. The photon stops currently used in the ID beamline FEs are designed to handle radiation power generated with $100 \mathrm{~mA}$ of stored beam current. Upgrading all the ID FEs to handle the radiation power generated by $300 \mathrm{~mA}$ stored beam will require increased capital funds. Commensurably, higher power will also have to be handled by the beamline mechanical and optical components, and these upgrades will require additional $R \& D$ and funding support.

A comprehensive plan that includes these enhancements will be developed by XFD in the 1998-99 period in consultation with various CATs. Support for this plan will be sought from DOE's Presidential Science Facilities Initiative.

\subsection{New Collaborative Access Teams}

In order to support a new CAT, the APS has to obtain funds for instrumentation behind the shield wall, i.e., the ID and the beamline FEs, for each of the new sectors. At this time, three new CATs are preparing proposals for additional sectors: one for protein crystallography, the second for ultrahigh pressure measurements, and the third to support small industry. In addition, SRI-CAT is seeking a fourth sector at the APS for the development of polarization techniques that have not been well addressed by other CATs.

The commercial (COM)-CAT, which will perform work on behalf of small industry, is seeking funds for capital construction of its beamlines; its operations will be supported by the collection of service charges from its industrial customers. The COM-CAT proposal has now been submitted for funding to the state of Illinois. This proposal includes the funds required for XFD to build the $\mathrm{ID}$ and the FEs for COM-CAT. Future CAT proposals will include a similar arrangement so that the funds for equipment behind the shield wall will not have to be sought separately.

\subsection{Strategic Plans for Dedicated Beamlines}

As pointed out above, 14 sectors of the APS are still available for experimental programs. The development of these sectors is consistent with DOE's mandate to fully and effectively utilize currently operating facilities before considering additional projects. This initiative hence addresses more opportunities for exploring new scientific research fields using dedicated beamlines.

Currently, 20 of the 34 APS sectors are assigned to various CATs. Each sector utilizes a generic set of techniques for using the $\mathrm{x}$-ray beam for the investigation of various science and technology problems. This approach has precluded specialized beamlines dedicated to one (often complex) technique, which can address some of the frontier areas of research. For these cases, the user community would prefer dedicated instruments. 
The dedicated beamlines will be chosen from the list presented below. For each beamline, we present a strategic analysis to aid the selection. The final selection of beamlines will be based on the level of interest of the respective user groups. For each beamline, funds will be jointly requested (from various sources including federal agencies and industry) by the APS and the beamline user group. Some of the beamlines require new R\&D effort, and the R\&D for some are currently supported through LDRD funds.

Funds for the construction and operation of these beamlines will be sought from DOE's Presidential Science Facilities Initiative, with the intent of completing 1-2 beamlines per year.

\section{BEAMLINE 1: Very High X-ray Energy Scattering (ID)}

The basic purpose of this beamline would be to perform the following studies in the $50-150 \mathrm{keV}$ energy range:

- Compton scattering studies of electron momentum distribution

- High-Q diffraction from molecular solids, glasses, and liquids to study structural details down to $0.1 \AA$

- Small angle scattering from bulk solids to study precipitate phases, voids or defects, etc.

- Structural studies of composite materials and fluids in confined geometries
- Studies of solid/liquid interfaces, corrosion, etc.

Currently no beamlines of this type have been planned at the APS, ESRF, or SPring-8. This is a new research opportunity, and a demonstration will be performed during FY1997-98 on the 1-ID-A (SRI-CAT) beamline.

For this program to succeed, development of a superconducting undulator is required. XFD has the world's best expertise in this area, and the preliminary design for a prototype device to be built jointly with Novosibirsk is complete. The prototype will be tested on an SRI-CAT beamline.

\section{BEAMLINE 2: Static and Dynamic Small Angle X-ray Scattering (ID)}

The basic purpose of this beamline would be to serve as a national facility to study length scales from $10 \AA$ to $10 \mu$, with the capability for anomalous small angle $\mathrm{x}$-ray scattering (SAXS) experiments down to millisecond time resolution using area detectors. The beamline will be of interest to the condensed matter physics, materials sciences, and biology communities.

Currently a BM beamline at the ESRF is used for biological studies and has capabilities similar to those at the NSLS. At the APS, two of the CATs (CMC and DND) have planned limited capabilities, with about $10-20 \%$ of their beam time available for SAXS experiments. SPring- 8 has yet to plan a facility. The need and unique ability to probe length scales using SAXS at a third-generation facility was emphasized by the Eisenberger-Knotek report of 1983. The leadership in the U.S. can bring together an active group of researchers in 
different disciplines to use this dedicated beamline.

\section{BEAMLINE 3: Sub-Nanosecond Temporal Resolution Studies (ID)}

The basic purpose of this beamline would be to use high brilliance, timing detectors, and the bunch structure of the storage ring for studies of conformational changes, chemical reactions, phase transitions, and defect propagation. The potential applications are:

- Chemical reactions on catalyst surfaces and supports

- Polymer morphology under applied stress

- Metallurgical phase changes under processing conditions

- Conformational changes in proteins

- Pump-probe studies

The beamline will be of interest to the condensed matter physics, chemical and materials sciences, bioscience, and industrial communities.

Currently at the APS, limited work is planned with instruments designed for select problems. The applications include dynamics of biomolecules (Bio-CARS), photosynthesis (BESSRC), fiber crystallization (DND), and nuclear coherent diffraction (SRI). The program was highlighted in the EisenbergerKnotek report but has not received the attention it deserves.
Key $R \& D$ required for the program to succeed includes the development of timing detectors, mechanical and optical techniques to sort photons from bunches, and synchronization techniques between the storage-ring bunch pattern, pump signal and probe technique, and the development of stroboscopic methods. The subject is a key candidate for future LDRD support.

\section{BEAMLINE 4: Coherence and Interference Techniques (ID)}

The basic purpose of this beamline would be to utilize the coherence of radiation in the $\mathrm{x}$-ray range from undulator sources. Fundamental understanding of the coherence and its use will be the focus for this beamline. Some of the studies include:

- Dynamics of complex fluids

- Surface diffusion

- Relaxation modes in glasses and liquids

- Fundamental physics of x-ray coherence and interference

Major users of this beamline will be from the condensed matter physics and materials sciences communities.

The importance of coherence was realized only recently. Three CATs at the APS (IMM, MHATT, and SRI) plan to perform basic studies to establish this important field of research. The new opportunities in this area are yet to be fully explored. 
For this effort to succeed, an immediate need is an understanding of $\mathrm{x}$-ray coherence and how it can be propagated through the various optical components of a beamline without being destroyed. This work is being undertaken by some of the APS CATs, particularly IMM and SRI. This field is now in the exploratory phase. It is already clear that coherence studies cannot be seriously pursued on multipurpose beamlines. By 1998, we expect that the requirements for a dedicated beamline will be well defined.

It is expected that the planned work at the APS and the LDRD-supported research will lead to many new applications of $\mathrm{X}$-ray coherence and, in turn, a new dedicated user community. This beamline is even more important when we realize that $x$-ray coherence is a prime basis for fourth-generation sources.

\section{BEAMLINE 5: Three-Dimensional Imaging (ID)}

This beamline would be devoted to threedimensional holographic and tomographic studies with hard x-rays. An x-ray holographic technique in the hard $\mathrm{x}$-ray range has yet to be developed and will open new areas of application. Applications for tomography down to $100 \AA$ spatial resolution will be found in biology, materials sciences, and engineering. The research focus will be in the areas of catalysis, corrosion science, and electronic components, with some problems demanding real-time studies.

The biology, condensed matter physics, and materials sciences and industrial communities will be the principal users of this beamline.
The initial work on holographic imaging has been carried out by SRI-CAT at long wavelengths. This work was supported by LDRD funds. In the hard $x$-ray range, the technique has yet to be established, and R\&D will be performed by SRI-CAT. No such effort is planned anywhere else. There have been many attempts (NSLS, Geo-CARS and CMC-CAT) to perform tomography of special materials; however, there are no dedicated efforts planned at the APS. More importantly, the proposed spatial resolution of the real-time measurements is unique to this beamline. Imaging received very high recognition in the Eisenberger-Knotek report, and the proposed beamline would fulfill this expectation.

Key $R \& D$ required for the program to succeed is the development of timing 2-D detectors and holography techniques. In addition, rapid image data reduction techniques need to be developed for effective implementation of tomographic imaging. The support to begin this software development was recently awarded through a Grand Challenge Grant from DOE to XFD, GeoCARS-CAT, and Argonne's Mathematics and Computer Science Division.

\section{BEAMLINE 6: Advanced Materials Engineering Research (ID)}

The proposed beamline will provide three unique capabilities for the processing of advanced materials:

- high-power x-ray beams from undulators

- capability to focus the high power to micron-size areas using appropriate optics 
- sufficient $\mathrm{x}$-ray penetration to deposit this high power density deep inside the materials being processed

In addition, $\mathrm{X}$-ray assisted processes, such as chemical vapor deposition (CVD) and etching, will be investigated.

The principal users will be from university engineering departments, DOD, the national labs, and industry.

This is a new program that will have a major impact on engineering fields. Basic expertise exists in the Beamline Engineering Group in XFD. However the initial work will be pursued in collaboration with university engineering departments.

The research will be started in FY 1998 with LDRD support. It is expected that even the preliminary results will attract a large number of materials engineers who can form the core group. In addition, many outreach activities to educate engineering departments about the capabilities of the APS will be pursued during 1998. Key $R \& D$ required to establish the program will be performed on an SRI-CAT beamline.

\section{BEAMLINE 7: Micro-Component Fabrication (BM)}

This beamline will exploit the special properties of a APS BM source, including high energy, high flux, and a high degree of collimation, to perform deep x-ray lithography (DXRL) for micromachining. The fabrication applications of micromechanical parts include micromotors, gear chains, x-ray optics, and pressure sensors. The beamline is expected to support internal and external client needs.
There is already significant interest in this beamline from industry, NASA, the national labs, and APS users.

The R\&D work on DXRL has been carried out by SRI-CAT with the support of LDRD funds. The DXRL technique is being evaluated for resolution, depth of exposure, aspect ratio, and throughput. Industrial partners are collaborating with XFD in developing the processes. Most European activity in this area is being carried out at the Forschungszentrum in Karlsruhe. No such work is planned by any of the APS CATs.

Key techniques required for the program to succeed are currently being developed by XFD. The industrial outreach activity will be increased as soon as a few prototype devices are made with the SRI-CAT beamline.

A marketing plan will be developed early in 1998 , and a marketing workshop of potential clients from universities, industry, and the national labs will be organized soon after to form the core community.

\section{BEAMLINE 8: Archaeometallurgy Research (BM)}

This beamline will be developed for the needs of a new community of x-ray users, the archaeometallurgists. The interest of this group is now nucleating at the APS with a collaboration between the Oriental Institute of The University of Chicago and XFD. The focus of the beamline is to provide analysis and composition of archaeometallurgical samples to provide insight into the metallurgical processes used in ancient times, primarily during the Bronze Age. The capabilities of the APS BM sources are well 
suited for these users. The beamline is expected to support international user groups.

Contacts include the Society of ArchaeoTec, the Ancient Metallurgy Research Group, and the Historical Metallurgy Society.

Our understanding of ancient (e.g., early Bronze Age) technological advances is both amazing and intriguing. However, very limited physical methods have been used to date to evaluate the industrial processes used, such as metal extraction and smelting. The $\mathrm{x}$-rays from the APS provide a unique opportunity for this potential group of users. No such effort has been planned at any other synchrotron radiation facility. The unique complimentarity of the expertise at the Oriental Institute and the XFD would be exploited by this beamline proposal.

Key techniques required for the program to succeed are currently being developed by XFD (SRI-CAT) jointly with the Oriental Institute. The core community of archaeometallurgists has been identified.

\section{BEAMLINE 9: X-ray Imaging for Medical Diagnostics Research (ID)}

This beamline would be devoted to the development of new tools for non-invasive imaging medical diagnostics. Some of the examples of imaging include coronary angiography, mammography, and specific antigenic foci (breast cancer, melanoma, or lymphoma). The purpose of the facility is only to develop the techniques for imaging various subjects, including human organs.

The initial work in this field was started at Stanford University, and currently the main focus is at the NSLS, where much of the work on both angiography and mammography is being carried out. A beamline dedicated to medical imaging is under construction at the ESRF and will begin operation in summer 1997. Many past attempts to begin this activity at the APS have not matured, primarily owing to a lack of leadership from the medical community. Imaging received very high recognition in the Eisenberger-Knotek report, and the proposed beamline would fulfill this expectation.

Key $R \& D$ required for the program to succeed has taken place at the Stanford Synchrotron Radiation Laboratory (SSRL), HASYLAB, and the NSLS. Further developmental work in the area of 2-D detectors will impact the field in a major way. Networking with hospitals (e.g., $\mathrm{U}$ of $\mathrm{C}$ ) at the highest level of ANL management is required to find the necessary leadership for this beamline.

Once the leadership for this beamline effort has been identified, a workshop for potential users from hospitals and the national labs will be organized.

A bonus for such a beamline would be the ability to perform 3-D computer tomography of other large objects of special interest, such as ancient Egyptian mummies or turbine blades.

\section{BEAMLINE 10: Radiation Therapy Research (BM)}

This beamline would be devoted to studies on radiation therapy (and radiosurgery) procedures. The unique capability of microfocusing optics for X-rays (e.g., as developed by XFD) provides a unique opportunity to expose only the affected area for treatment. The technique 
can be used concurrently to perform microtomography of the affected area to plan the treatment.

While radiation therapy is an established field of research at medical laboratories, the use of synchrotron radiation is totally new, and hence much effort will be required to attract interested medical researchers to a synchrotron and to evaluate the advantages of a strong radiation source.

Initial work at the APS must be carried out using future LDRD or similar program development funds in collaboration with researchers from The University of Chicago. Networking with hospitals (e.g., U of C) to find the necessary leadership for this beamline is the first step, followed by collaborative research. Key R\&D efforts required for the program to succeed at a synchrotron radiation source can then be established.

\section{BEAMLINE 11: Molecular Environmental Science (ID)}

This beamline would be devoted to the special needs of the community performing research in the field of molecular environmental science. The community focuses on:

- Speciation, reactivity, and mobility of contaminants in aqueous solutions and their relationship to molecular biological processes

- The role of surfaces and interfaces in molecular environmental science

- The need for handling radioactive samples at synchrotron radiation facilities
Initial work in the area of environmental science has been done at the NSLS and SSRL; more work is planned at the Advanced Light Source (ALS) and APS. A 1995 workshop organized by DOE concluded that the planned beamlines at the APS (MR-CAT, GeoCARSCAT, and PNC-CAT) "will not likely meet the increased demand after 1998 because of anticipated needs for service work on environmental samples." Future demand and the requirement for studying highly toxic and radioactive samples will dictate the need for a dedicated beamline at the APS with special environment, safety, and health protection features. There is a lab-wide interest in this area of research, and LDRD funds were provided to the ER Division in FY 1997.

Key $R \& D$ required for the program to succeed is in place. The implementation of the proposed beamline, however, requires major planning for safe handling of samples and their disposal. ANL has the best expertise and facilities (e.g., in the Bldg. $200 \mathrm{M}$-wing) for handling radioactive samples and, in particular, actinides.

\section{BEAMLINE 12: X-ray Micro- fluorescence, Microimaging \& Microdiffraction (ID)}

The basic purpose of this beamline would be to perform both scanning and real-time studies of submicron-size areas using microprobe techniques. Applications of these tools in materials sciences, industrial fabrication, and biological systems are attracting very diverse users. Attention has also been focused on residual stress analysis and crack propagation in materials and recently on the capability of studying the dynamics of small particles using microfluorescence. 
This beamline will attract scientists from the condensed matter physics, materials sciences, biology, soil science, environmental science, defense research, and industrial research communities.

Currently the techniques for submicron focusing are being developed by SRI-CAT. GeoCARS-CAT will set aside approximately $1 / 3$ of the time on one of its beamlines for dedicated users from the soil science community. By 1999, UNI-CAT will build a beamline that will be dedicated roughly halftime to these studies. At the ESRF, a new beamline was added with the sole purpose of using microprobe techniques. The SRI-CAT strategic instrument station is already oversubscribed.

This is an easy area to market. The demand is now growing. The techniques are being refined by SRI-CAT. To support this program, XFD will continue to develop focusing optics (e.g., zone plates). The need for 2-D detector development will be crucial for realtime studies.

\subsection{Strategic Plan for FEL Undulator Development}

The basic purpose of this XFD effort is to design and build an extra long undulator, along with both particle and photon diagnostics, as a part of the APS FEL initiative. Production of $50-100 \mathrm{~nm}$ radiation is the prime objective of this project.

The availability of linac and accelerator expertise in ASD are prerequisites to this plan. XFD has the world's best expertise in the construction of magnetic devices, and this has been enhanced by collaboration with scientists from Novosibirsk. Concurrently, some demonstration experiments on this unique source will be performed.

Brookhaven National Laboratory has planned a similar capability based on a different principle of seeding the laser. SSRL has plans for a $0.1 \mathrm{~nm}$ FEL. The potential sponsor for the APS effort is DOE.

Key $R \& D$ required for the program to succeed is the development of a long undulator that includes the required diagnostics. XFD has performed all computations on the gain lengths to demonstrate this capability, and prototype undulators have been designed. These will be built in collaboration with Novosibirsk to reduce costs of fabrication. Funding for this activity has been made available from LDRD funds. The prototype device for testing the SASE concept will be ready early in 1998.

A parallel study on the transfer of coherence through beamline optics has been undertaken by SRI-CAT. More work is planned for 1998 to address the thermal problems related to the nanosecond power train from an FEL in the $\mathrm{x}$-ray energy regime.

\subsection{LDRD Program in Support of Long-Term Goals}

The support needed to perform the $R \& D$ to prepare for the long-term goals described above is generally not provided through the DOE annual budget process. The main source of support for such $R \& D$ activities is derived from LDRD funds. Table 6.1 lists the LDRD programs funded for the 1996-98 period in support of the long-term goals of XFD. 
Table 6.1 Current LDRD Programs in Support of the Long-Term Goals of XFD

Long Term Program

LDRD Program

1. FE Upgrade

2. Micromachining Beamline

3. Imaging/Coherence Beamline

4. Ultrahigh Energy-Resolution Beamline

5. X-ray FEL
1. CVD Diamond Imaging Detector (97-98)

2. Deep Etch Lithography (96-98)

3. Intensity Fluctuation Spectroscopy (96-98)

Heterodyne Correlation Spectroscopy (96-98)

4. Fabry-Perot Cavity for Sub-meV Resolution (97-99)

Anomalous Inelastic Scattering with meV Resolution (97-99)

5. Computational analysis to specify the requirements of an undulator to demonstrate the SASE concept, and prototype testing (97-99) 
6 LONG-TERM STRATEGIC PLANS FOR XFD 

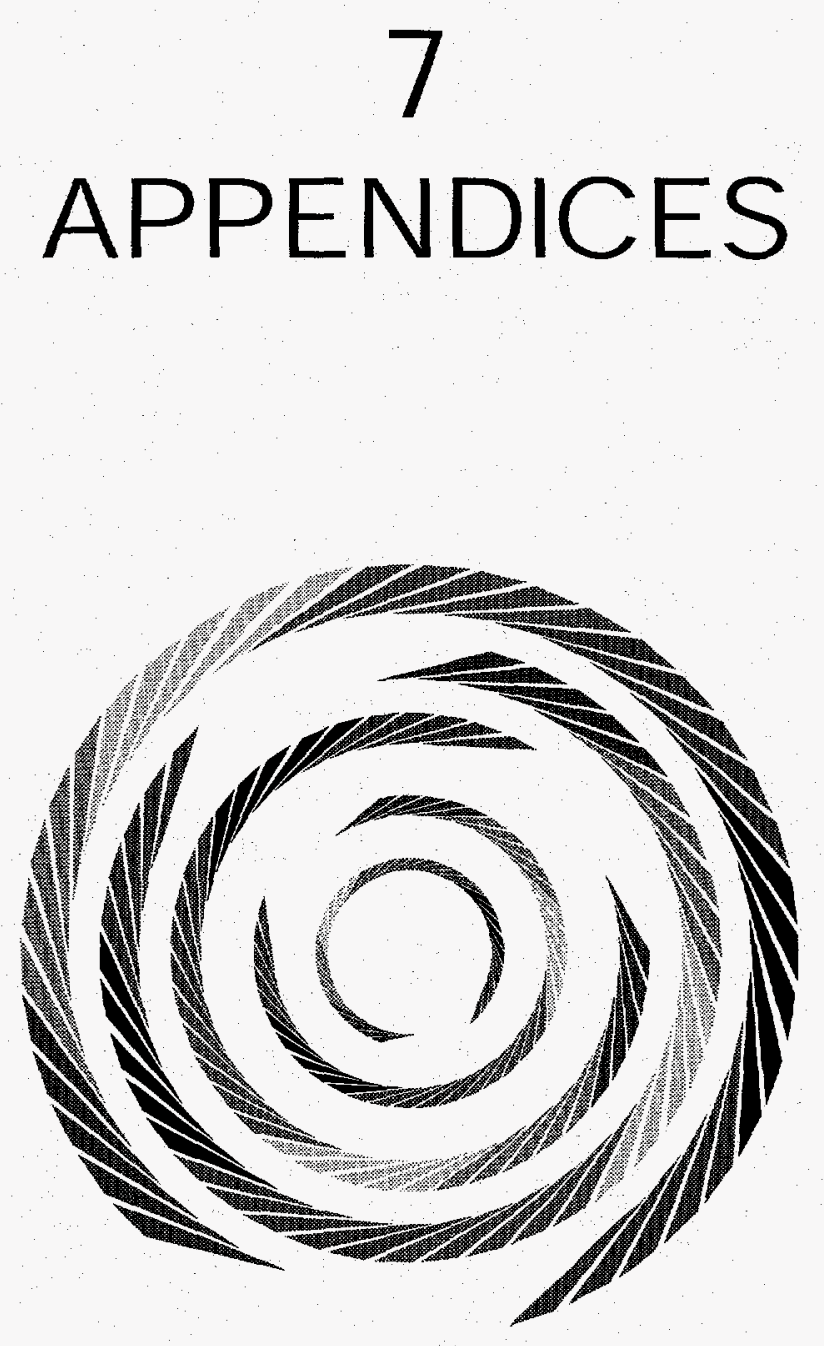


\section{Recent Publications by XFD Staff}

Agamalian, M., J. M. Drake, S. K. Sinha, and J. D. Axe, Neutron Diffraction Study of the Pore Surface Layer of Vycor Glass, Phys. Rev. E 55 (1997) 3021-3027

Alp, E. E., W. Sturhahn, and T. Toellner, Synchrotron Mossbauer Spectroscopy of Powder Samples, Nucl. Instrum. Meth. Phys. Res. B 97 (1995) 526-529

Alp, E. E., Report on the SRI '95 Workshop on the Timing Structure of the Advanced Photon Source, Rev. Sci. Instrum. 67 (9) (1996) CD-ROM

Amiri, A., K. Vafai, and T. M. Kuzay, Effects of Boundary Conditions on Non-Darcian Heat Transfer through Porous Media and Experimental Comparisons, Numerical Heat Transfer, Part A, 27 (1995) 651-664

Assoufid, L., W.-K. Lee, and D. M. Mills, A Finite Element Analysis of Room-Temperature Silicon Crystals for the Advanced Photon Source Bending-Magnet and Insertion-Device Beams, Rev. Sci. Instrum. 66 (1995) 2064

Assoufid, L., and A. M. Khounsary, Contact Heat Conductance at Diamond-OFHC Copper Interface with GaIn Eutectic as a Heat Transfer Medium, Rev. Sci. Instrum. 67 (9) (1996) CD-ROM

Assoufid, L., K. W. Quast, and H. L. T. Nian, Modeling of the Performance of a LiquidGallium-Cooled Silicon Monochromator for a High-Energy-Resolution Scattering Beamline Comparison with Experimental Data, SPIE 2855 (1996) 250-261

Barraza, J., D. Shu, and T. M. Kuzay, Support Systems for Optics in the Experimental Stations at the Advanced Photon Source, Rev. Sci. Instrum. 66 (1995) 1630-1632

Barraza, J., D. Shu, W. Yun, and T. M. Kuzay, Design of a High Precision Mirror-Rotation System at the Advanced Photon Source, Rev. Sci. Instrum. 67 (9) (1996) CD-ROM

Basdogan, I., T. J. Royston, A. A. Shabana, D. Shu, and T. M. Kuzay, Vibratory Response of a Mirror Support/Positioning System for the Advanced Photon Source Project at Argonne National Laboratory, SPIE Vol. 2865 Actuator Technology and Applications (1996) 2-14

Blasdell, R. C., and A. T. Macrander, Prototype Grooved and Spherically Bent Si Backscattering Crystal Analyzers for meV Resolution Inelastic X-ray Scattering, Rev. Sci. Instrum. 66 (1995) 2075-2077

Blasdell, R. C., L. A. Assoufid, and D. M. Mills, Diamond Monochromators for APS UndulatorA Beamlines, Argonne National Laboratory Report ANL/APS/TB-24 (1995) 
Brauer, S., B. Rodricks, L. Assoufid, M. Beno, and G. Knapp, Liquid-Metal-Cooled, CurvedCrystal Monochromator for Advanced Photon Source Bending-Magnet Beamline 1-BM, Rev. Sci. Instrum. 67 (9) (1996) CD-ROM

Bresloff, C., and D. M. Mills, The Advanced Photon Source Metrology Laboratory, Rev. Sci. Instrum. 67 (9) (1996) CD-ROM

Brite, C., D. Shu, T. Nian, Z. Wang, D. Haeffner, E. Alp, R. Parry, and T. M Kuzay, Modular Filter Design for White-Beam Undulator/Wiggler Beamlines at the Advanced Photon Source, Rev. Sci. Instrum. 66 (1995) 1639-1642

Cai, Z., R. J. Dejus, P. Den Hartog, Y. Feng, E. Gluskin, D. Haeffner, P. Ilinski, B. Lai, D. Legnini, E. R. Moog, S. Shastri, E. Trakhtenberg, I. Vasserman, and W. Yun, APS Undulator Radiation - First Results, Rev. Sci. Instrum. 67 (9) (1996) CD-ROM

Cai, Z., W. Yun, and P. Plag, Parameter Optimization for Producing an Elliptical Surface from a Sperical Surface by Differential Deposition, SPIE Vol. 2516 (1995) 62-68

Cai, Z., B. Lai, W. Yun, E. Gluskin, D. Legnini, P. Illinski, and G. Srajer, Beam Size Measurement of Stored Electron Beam at the APS Storage Ring Using Pinhole Optics, Rev. Sci. Instrum. 67 (9) (1996) CD-ROM

Cai, Z., B. Lai, W. Yun, E. Gluskin, R. Dejus, and P. Ilinski, Phase-Space Measurement of Stored Electron Beam at the Cornell Electron Storage Ring Using a Combination of Slit Array and CCD Detector, Rev. Sci. Instrum. 66 (1995) 1859-1861

Chang, J., D. Shu, T. Nian, T. M. Kuzay, and P. K. Job, Design of Integral Shutters for the Beamlines of the Advanced Photon Source, Rev. Sci, Instrum. 66 (1995) 1648-1650

Chang, J., D. Shu, G. Decker, T. Nian, T. M. Kuzay, A. Lumpkin, and X. Wang, Mechanical Design of a Missteered Beam Safety Monitor at the Advanced Photon Source, Rev. Sci. Instrum. 67 (9) (1996) CD-ROM

Dejus, R. J., I. Vasserman, E. R. Moog, and E. Gluskin, Phase Errors and Predicted Spectral Performance of a Prototype Undulator, Rev. Sci. Instrum. 66 (1995) 1875-1877

Dejus, R. J., and M. Sanchez del Rio, XOP: A Graphical User Interface for Spectral Calculations and X-ray OPtics Utilities, Rev. Sci. Instrum. 67 (9) (1996) CD-ROM

Den Hartog, P., J. Grimmer, T. Klippert, E. Trakhtenberg, and S. Xu, Design of the Vacuum System for the Elliptical Multipole Wiggler at the Advanced Photon Source, Rev. Sci. Instrum. 67 (9) (1996) CD-ROM

Deutsch, M., X. Z. Wu, E. B. Sirota, S. K. Sinha, B. M. Ocko, O. M. and Magnussen, Crystalline Bilayers on the Surface of Molten Alcohols, Europhys. Lett. 30 (1995) 283 
Dimowski, W., R. McQueeney, T. Egami, Y. P. Feng, S. K. Sinha, T. Hinatsu, and S. Uchida, Temperature-Dependent X-ray Diffuse Scattering from Single Crystals of $\mathrm{La}-\mathrm{Sr}-\mathrm{Cu}-\mathrm{O}$, Phys. Rev. B 52 (1995) 6829

Feng, Y. P., B. Lai, I. McNulty, R. J. Dejus, K. J. Randall, and W. Yun, Beam Transport Radiation Shielding for Branch Lines 2-ID-B and 2-ID-C, Argonne National Laboratory Report LS-251 (1995)

Feng, Y. P., S. K. Sinha, E. E. Fullerton, G. Grübel, D. Abernathy, D. P. Siddons, and J. B. Hastings, X-ray Fraunhofer Diffraction Patterns from a Thin-Film Waveguide, Appl. Phys. Lett. 67 (1995) 3647-3649

Feng, Y. P., S. K. Sinha, C. A. Melendres, and D. D. Lee, X-ray Off-Specular Scattering Studies of Electrochemical Pitting of Cu Surfaces in Sodium Bicarbonate Solution, Physica B 221 (1996) 251

Fernandez, P. B., Silicon Bonding Techniques for X-ray Optics, Argonne National Laboratory Report LS-249 (1995)

Frachon, D., I. Vasserman, P. M. Ivanov, E. A. Medvedko, E. Gluskin, and N. A. Vinokurov, Magnetic Measurements of the Elliptical Multipole Wiggler Prototype, Argonne National Laboratory Report ANL/APS/TB-22 (1995)

Friedman, N., J. Hawkins, D. Travis, and G. Laurence, Front End Equipment Protection System at the Advanced Photon Source, Rev. Sci. Instrum. 67 (9) (1996) CD-ROM

Frisken, B. J., D. S. Cannell, M. Y. Lin, and S. K. Sinha, Neutron Scattering Studies of Binary Mixtures in Silica Gels, Phys. Rev. E 51 (1995) 5866

Gibaud, A., J. Wang, M. Tolan, G. Vignaud, and S. K. Sinha, An X-ray Scattering Study of Laterally Modulated Structures: Investigation of Coherence and Resolution Effects with a Grating, J. de Physique I, France 6 (1996) 1085

Gluskin, E., K. J. Randall, I. McNulty, W. Yun, A. M. Khounsary, and B. Lai, Soft X-ray Instrumentation and its Applications at the Advanced Photon Source, Journal of X-ray Science and Technology 5 (1995) 29-42

Gluskin, E., Elliptical Multipole Wiggler for the Production of Variably Polarized Radiation, Rev. Sci. Instrum. 67 (9) (1996) CD-ROM

Gottschalk, S., D. C. Quimby, K. E. Robinson, E. Moog, and R. Apparao, Central Field Design Methods for Hybrid Insertion Devices, Rev. Sci. Instrum. 67 (9) (1996) CD-ROM

Gottschalk, S., K. Robinson, I. Vasserman, R. Dejus, and E. Moog, End Field Design and Tuning Methods for Insertion Devices, Rev. Sci. Instrum. 67 (9) (1996) CD-ROM 
Gottschalk, S., K. E. Robinson, D. C. Quimby, K. W. Kangas, I. Vasserman, R. Dejus, and E. Moog, Multipole and Phase Tuning Methods for Insertion Devices, Rev. Sci. Instrum. 67 (9) (1996) CD-ROM

Haddad, W. S., J. E. Trebes, D. M. Goodman, H.-R. Lee, I. McNulty, E. H. Anderson, and A. O. Zalensky, Ultrahigh-Resolution Soft X-ray Tomography, SPIE Proc. 2516 (1995) 102

Hawkins, J., C. Seaver, J. Stein, J. Stoffel, and N. Friedman, Personnel Safety System for the Beamlines at the Advanced Photon Source, Rev. Sci. Instrum. 67 (9) (1996) CD-ROM

Illinski, P., W. Yun, B. Lai, E. Gluskin, and Z. Cai, Measurements of X-ray Spectral Flux of High-Brightness Undulators by Gas Scattering, Rev. Sci. Instrum. 66 (1995) 1907-1909

Ilinski, P., R. J. Dejus, E. Gluskin, and T. I. Morrison, Some Practical Aspects of Undulator Radiation Properties, SPIE Vol. 2856 Optics for High-Brightness Synchrotron Radiation Beamlines II (1996) 16-25

Job, P. K., and H. J. Moe, Dose Rate Estimates in the First Optical Enclosure due to Particle Beam Loss in the Insertion Device Transition Region during Injection, Argonne National Laboratory Report LS-243 (1995)

Job, P. K., and D. R. Haeffner, Secondary Bremsstrahlung Dose Rates from Glancing Incidence Targets, Rev. Sci. Instrum. 67 (9) (1996) CD-ROM

Job, P. K., H. Moe, T. A. Gabriel, J. Drischler, and R. A. Lillie, Shielding Optimization Studies at the Advanced Photon Source for Multi-Radiation Environment due to Partial Beam Loss, Proc. ANS 1996 Topical Meeting on Radiation Protection and Shielding, Cape Cod, MA, April 1996, p. 276

Khounsary, A. M., and W. Yun, On Optimal Contact Cooling of High-Heat-Load X-ray Mirrors, Rev. Sci. Instrum. 67 (9) (1996) CD-ROM

Khounsary, A., W. Yun, E. Trakhtenberg, S. Xu, L. Assoufid, and W.-K. Lee, Contact-Cooled U-Monochromator for High Heat Load X-ray Beamlines, SPIE High Heat Flux Eng. Conf. Proc. 1996

Knapp, G. S., C. S. Rogers, M. A. Beno, C. C. Wiley, G. Jennings, and P. L. Cowan, Cryogenic Monochromators as a Solution to Undulator Heat Loads at Third Generation Synchrotron Sources, Rev. Sci. Instrum. 66 (1995) 2138-2140

Krasnicki, S., The APS Topography Station, Rev. Sci. Instrum. 67 (9) (1996) CD-ROM

Krasnoperova, A., Z. Chen, F. Cerrina, E. DiFabrizio, M. Gentili, W. Yun, B. Lai, E. Gluskin, Microfocusing Optics for Hard X-Rays Fabricated by X-Ray Lithography, SPIE Proc. 2516 (1995) 15 
Kushnir, V. I., and A. T. Macrander, Experimental Station for Inelastic Scattering Spectroscopy of Electronic Excitations, Rev. Sci. Instrum. 67 (9) (1996) CD-ROM

Kushnir, V. I., and M. Popovici, Bent Crystal Analyzer without Grooves for Inelastic X-ray Scattering, Rev. Sci. Instrum. 67 (9) (1996) CD-ROM

Kuzay, T. M., and T. Warwick, Innovations in the Design of Mechanical Components for a Beamline - The SRI '95 Workshop 2 Summary, Rev. Sci. Instrum. 67 (9) (1996) CD-ROM

Kuzay, T. M., H. L. T. Nian, Z. Wang, and D. Shu, Performance Analysis of the Commissioning Filter and Window Assembly for Insertion Device Beamlines at the Advanced Photon Source, Rev. Sci. Instrum. 67 (9) (1996) CD-ROM

Lai, B., W. Yun, Y. Xiao, L. Yang, Z. Cai, A. Krasnoperova, F. Cerrina, E. DiFabrizio, L. Luciani, M. Figliomeni, and M. Gentili, Development of a Hard X-ray Imaging Microscope, Rev. Sci. Instrum. 66 (1995) 2287-2289

Lai, B., D. C. Mancini, W. Yun, and E. Gluskin, Beamline and Exposure Station for Deep X-ray Lithography at the Advanced Photon Source, SPIE Proc. 2880 (1996) 171

Lang, J. C., and G. Srajer, Bragg Transmisssion Phase Plate for the Production of Circularly Polarized X-rays, Rev. Sci. Instrum. 66 (1995) 1540-1542

Lang, J. C., G. Srajer, and R. J. Dejus, A Comparison of an Elliptical Multipole Wiggler and Crystal Optics for the Production of Circularly Polarized X-rays, Argonne National Laboratory Report LS-245 (1995)

Lang, J. C., G. Srajer, C. Detlefs, A. I. Goldman, H. Konig, X. Wang, B. N. Harmon, and R. W. McCallum, Confirmation of Quadrupolar Transitions in Circular Magnetic X-ray Dichroism at the Dysprosium LIII Edge, Phys. Rev. Lett. 74 (1995) 4935-4938

Lang, J. C., G. Srajer, and R. J. Dejus, A Comparison of an Elliptical Multipole Wiggler and Crystal Optics for the Production of Circularly Polarized X Rays, Rev. Sci. Instrum. 67 (1996) 62-67

Lee, W.-K., P. B. Fernandez, T. Graber, and L. Assoufid, High-Heat-Load Synchrotron Tests of Room-Temperature Silicon Crystal Monochromators at the CHESS F-2 Wiggler Station, Argonne National Laboratory Report LS-250 (1995)

Lee, W.-K., D. M. Mills, L. Assoufid, R. C. Blasdell, P. B. Fernandez, C. S. Rogers, and R. K. Smither, High Heat Load Monochromator Development at the Advanced Photon Source, Optical Engineering 34 (1995) 418-425

Lee, W.-K., R. C. Blasdell, P. B. Fernandez, A. T. Macrander, and D. M. Mills, Brilliance and Flux Reduction in Imperfect Inclined Crystals, Rev. Sci. Instrum. 67 (9) (1996) CD-ROM 
Li, Y., D. Ryding, C. Liu, T. M. Kuzay, M. W. McDowell, and R. A. Rosenberg, X-ray Photoelectron Spectroscopy Analysis of Cleaning Procedures for Synchrotron Radiation Beamline Materials at the Advanced Photon Source, J. Vac. Sci. Technol. 13 (1995) 576580

Li, Z., W. Zhao, J. Quinn, M. H. Rafavlovich, J. Sokolov, R. B. Lennox, A. Eisenberg, X. Z. Wu, M. W. Kim, S. K. Sinha, M. and Tolan, X-ray Reflectivity of Diblock Copolymer Monolayers at the Air-Water Interface, Langmuir 11, (1995) 4785

Lin, M. Y., H. J. M. Hanley, S. K. Sinha, G. C. Straty, D. G. Peiffer, M. W. and Kim, A Small Angle Neutron-Scattering Study of Worm-like Micelles, Physica B 213 and 214 (1995) 613

Lin, M. Y., H. J. M. Hanley, S. K. Sinha, G. C. Straty, D. G. Peiffer, M. W. and Kim, ShearInduced Behavior in a Solution of Cylindrical Micelles, Phys. Rev. E Rapid Communications 53 (1996) R4302

Liu, C., R. W. Nielsen, T. L. Kruy, D. Shu, and T. M. Kuzay, Vacuum Tests of a Beamline Front End Mock-up at the Advanced Photon Source, Rev. Sci. Instrum. 66 (1995) 27032707

Liu, C., D. Shu, T. M. Kuzay, and R. Kersevan, Monte Carlo Simulations of the Vacuum Performance of Differential Pumps at the Advanced Photon Source, Rev. Sci. Instrum. 67 (9) (1996) CD-ROM

Liu, C., D. Ryding, R. W. Nielsen, T. L. Kruy, and T. M. Kuzay, Cleaning and Outgassing Studies of Machinable Tungsten for Beamline Safety Shutters, Rev. Sci. Instrum. 67 (9) (1996) CD-ROM

Liu, C., J. T. Collins, R. W. Nielsen, T. L. Kruy, M. Ramanathan, and T. M. Kuzay, Importance of Components Cleaning in the Ultrahigh Vacuum Performance of Beamline Front Ends, Rev. Sci. Instrum. 67 (9) (1996) CD-ROM

Lynn, J. W., Q. Huang, S. K. Sinha, Z. Hossain, L. C. Gupta, R. Najarajan, and C. Godard, Neutron Scattering Study of AntiFerromagnetic Order in the Magnetic Superconductors $\mathrm{RNi}_{2} \mathrm{~B}_{2} \mathrm{C}$, Physica B 224 (1996) 66

Macrander, A. T., V. I. Kushnir, and R. C. Blasdell, Performance of Spherically Focusing $\mathrm{Ge}(444)$ Backscattering Analyzers for Inelastic X-ray Scattering, Rev. Sci. Instrum. 66 (1995) 1546-1548

Macrander, A. T., R. C. Blasdell, P. A. Montano, and C. C. Kao, Inelastic X-ray Scattering from $6 \mathrm{H}-\mathrm{SiC}$, in Wide Bandgap Semiconductors and Devices, ed. F. Ren et al., Proceedings of the Electrochemical Society, Volume 95-21 (1995) 98

Macrander, A. T., and P. M. Platzman, Alternative Methods for sub-eV Resolution Inelastic X-ray Scattering, Rev. Sci. Instrum. 67 (9) (1996) CD-ROM 
Macrander, A. T., M. Saginuri, S. Yao, P. J. Hesketh, and C. J. Bresloff, Possible Use of Direct Silicon-on-Silicon Bonding for Construction of Analyzers for Inelastic Scattering, Rev. Sci. Instrum. 67 (9) (1996) CD-ROM

Macrander, A. T., P. A. Montano, D. L. Price, V. I. Kushnir, R. C. Blasdell, C. C. Kao, and B. R. Cooper, Inelastic X-ray Scattering from TiC and Ti Single Crystals, Phys. Rev. B 54 (1996) 305-312

Makarov, O. A., Z. Chen, A. A. Krasnoperova, F. Cerrina, V. V. Cherkashin, A. G. Poleschuk, and V. P. Koronkevich, A New Application for X-ray Lithography: Fabrication of Blazed Diffractive Optical Elements with a Deep Phase Profile, in Electron-Beam, X-ray, EUV, and Ion-Beam Submicrometer Lithographies for Manufacturing VI, ed. D. E. Seeger, Proc. SPIE Vol. 2723 (1996) 261-267

McNulty, I., Y. P. Feng, W. S. Haddad, and J. E. Trebes,Tomographic Scanning Microscope for 1-4 keV X-rays, SPIE Proc. 2516 (1995) 217

McNulty, I., W. S. Haddad, J. Trebes, and E. H. Anderson, Soft X-ray Scanning Microtomography with Submicrometer Resolution, Rev. Sci. Instrum. 66 (1995) 1431-1433

McNulty, I., A. Khounsary, Y. P. Feng, Y Qian, J. Barraza, C. Benson, and D. Shu, A Beamline for 1-4 keV Microscopy and Coherence Experiments at the Advanced Photon Source, Rev. Sci. Instrum. 67 (9) (1996) CD-ROM

Melendres, C. A., Y. P. Feng, D. Lee, and S. K. Sinha, X-ray Diffuse Scattering for the in situ Study of Electrochemically Induced Pitting on Metal Surfaces, J. Electrochem. Soc. 142 (1995) L19

Mills, D. M., A. Freund, and T. Ishikawa, Workshop Summary on Thermal Management of X-ray Optical Components for Synchrotron Radiation, Rev. of Sci. Instrum. 66 (1995) $2380-2382$

Mills, D. M., Cryogenically Cooled Monochromators for the Advanced Photon Source, Rev. Sci. Instrum. 67 (9) (1996) CD-ROM

Minich, J. M., Designing a Beamline Equipment Protection System Using a Programmable Logic Controller, Rev. Sci. Instrum. 67 (9) (1996) CD-ROM

Montano, P. A., G. S. Knapp, G. Jennings, E. Gluskin, E. Trakhtenberg, I. B. Vasserman, P. M. Ivanov, D. Frachon, E. R. Moog, L. R. Turner, G. K. Shenoy, M. J. Bedzyk, M. Ramanathan, M. A. Beno, and P. L. Cowan, Elliptical Multipole Wiggler Facility at the Advanced Photon Source, Rev. Sci. Instrum. 66 (1995) 1839-1841

Mooney, T. M., N. D. Arnold, B. K. Cha, K. A. Goetze, J. B. Kowalkowski, M. R. Kraimer, G. J. Nawrocki, D. R. Reid, J. P. Sullivan, D. B. Wallis, and J. R. Winans, Beamline Control and Data Acquisition Software, Rev. Sci. Instrum. 67 (1996) CD ROM 
Naya, J. E., P. von Ballmoos, F. Albernhe, G. Vedrenne, R. K. Smither, M. Faiz, P. B. Fernanadez, and T. Graber, Experimental Results Obtained with the PositronAnnihilation- Radiation Telescope of the Toulouse-Argonne Collaboration, in Imaging in High Energy Astronomy, ed. L. Bassani and G. di Cocco, (Kluwer Academic Publishers, Dordrecht, The Netherlands, 1995) pp. 313-317

Naya, J. E., P. von Ballmoos, R. K. Smither, M. Faiz, P. B. Fernandez, T. Graber, F. Albernhe, and G. Vedrenne, Experimental Results Obtained with the Positron-Annihilation- Radiation Telescope of the Toulouse-Argonne Collaboration, Nucl. Instrum. Meth. Phys. Res. A 373 (1996) 159-164

Nian, H. L. T., D. Shu, and T. M. Kuzay, Thermo-Mechanical Analysis of the White-Beam Slits for an Undulator Beamline at the Advanced Photon Source, Rev. Sci. Instrum. 66 (1995) 1735-1737

Nian, H. L. T., I. C. Sheng, D. Shu, and T. M. Kuzay, Thermo-Mechanical Analysis of the White Beam Slits for a Wiggler/Undulator Beamline at the Advanced Photon Source, Rev. Sci. Instrum. 66 (1995) 1846-1849

Nian, H. L. T., and T. M. Kuzay, Closed-Form Solutions for a Disk, a Filter, and a Window for an X-ray Beamline at the Advanced Photon Source, Rev. Sci. Instrum. 67 (9) (1996) CD-ROM

Nian, H. L. T., T. M. Kuzay, and I. C. Sheng, Thermal Buckling Parametric Analysis for a Diamond Disk for a X-ray Beamline at the Advanced Photon Source, Rev. Sci. Instrum. 67 (9) (1996) CD-ROM

Nian, H. L. T., T. M. Kuzay, and D. Shu, Design Analysis of a Composite L5-20 Slit for X-ray Beamlines at the Advanced Photon Source, SPIE Vol. 2855 High Heat Flux Engineering III (1996) 276-290

Nian, H. L. T., T. M. Kuzay, D. Shu, and C. Benson, Design Analysis of a Composite L5-20 Slit for X-ray Beamlines at the Advanced Photon Source, Rev. Sci. Instrum. 67 (9) (1996) CD-ROM

Nian, H. L. T., T. M. Kuzay, J. Collins, D. Shu, C. Benson, and R. Dejus, Thermo-Mechanical Analysis of a User Filter Assembly for Undulator/Wiggler Operations at the Advanced Photon Source, SPIE Vol. 2855 High Heat Flux Engineering III (1996) 262-275

Perahia, D., D. G. Wiesler, S. K. Satija, L. J. Fetters, S. K. Sinha, and G. S. Grest, Neutron Reflectivity of End Grafted Polymers in Poor Solvent: Kinetic Effects, Physica B 221 (1996) 337

Pflüger, J., G. Heintze, and I. Vasserman, Search for Possible Radiation Damage on a NdFeB Permanent Magnet Structure after Two Years of Operation, Rev. Sci. Instrum. 66 (1995) 1946-1948 
Qian, S., W. Jark, P. Z. Takacs, K. J. Randall, Z. Xu, and W. Yun, In-Situ Long Trace Profiler for Measurement of Mirror Profiles at Third-Generation Synchrotron Facilities, Rev. Sci. Instrum. 67 (9) (1996) CD-ROM

Quintana, J. P., V. I. Kushnir, and G. Rosenbaum, Synchrotron Experimental Results on an Unribbed Sagittally Focusing Crystal Monochromator, Nucl. Instrum. Meth. A 362 (1995) 592

Quintana, J. P., Y. Dolin, P. Georgopoulos, and V. I. Kushnir, Anticlastic Curvature Measurements on Unribbed Crystal Optics for Synchrotron Radiation, Rev. Sci. Instrum. 66 (1995) 2188-2189

Randall, K. J., E. Gluskin, and Z. Xu, Spectroscopy Beamline at the Advanced Photon Source for the Photon Energy Region from 0.5 to $3 \mathrm{keV}$, Rev. Sci. Instrum. 66 (1995) 4081-4086

Randall, K. J., J. Z. Xu, D. Graessle, J. J. Fitch, S. DiFonzo, B. R. Mueller, and W. Jark, Reflectivity Measurements of Multilayer Optics for Use on the Advanced Photon Source Soft X-ray Beamlines, NSLS Annual Report (1996)

Randall, K., Z. Xu, E. Gluskin, I. McNulty, R. Dejus, S. Krinsky, O. Singh, C.-C. Kao, E. D. Johnson, C. T. Chen, and G. Meigs, Characterization of a Novel Elliptically Polarized Wiggler, J. Electr. Spectr. \& Related Phenomena 80 (1996) 433-436

Rodricks, B., Time-Resolved X-ray Scattering Program at the Advanced Photon Source, Rev. Sci. Instrum. 66 (1995) 1456-1458

Rodricks, B., S. L. Barna, S. M. Gruner, J. A. Shepard, M. W. Tate, and R. L. Wixted, Pixel Array Detector for Time-Resolved X-ray Scattering, Rev. Sci. Instrum. 67 (9) (1996) CD-ROM

Rogers, C. S., and L. Assoufid, Design and Thermal Stress Analysis of High Power X-ray Monochromators Cooled with Liquid Nitrogen, Rev. Sci. Instrum. 66 (1995) 2200-2202

Rogers, C. S., D. M. Mills, W.-K. Lee,. G. S. Knapp, J. Holmberg, A. Fruend, M. Wulff, M. Rossat, M. Hanfland, and H. Yamaoka, Performance of a Liquid-Nitrogen-Cooled, Thin Silicon Crystal Monochromator on a High-Power, Focused Wiggler Synchrotron Beam, Rev. Sci. Instrum. 66 (1995) 3494-3499

Rogers, C. S., D. M. Mills, L. Assoufid, and T. Graber, Performance of Cryogenically Cooled, High-Heat-Load Silicon-Crystal Monochromators with Porous Media Augmentation, Rev. Sci. Instrum. 67 (9) (1996) CD-ROM

Rogers, C. S., D. M. Mills, W.-K. Lee, P. B. Fernandez, and T. Graber, Experimental Results with Cryogenically Cooled, Thin Silicon-Crystal X-ray Monochromators on High-Heat-Flux Beamlines, SPIE Vol. 2855 High Heat Flux Engineering III (1996) 170-179 
Rogers, C. S., D. M. Mills, P. B. Fernandez, G. S. Knapp, M. Wulff, M. Hanfland, M. Rossat, A. Freund, G. Marot, J. Holmberg, and H. Yamaoka, Further Tests on Liquid-NitrogenCooled, Thin Silicon-Crystal Monochromators Using a Focused Wiggler Synchrotron Beam, Rev. Sci. Instrum. 67 (9) (1996) CD-ROM

Shastri, S. D., R. J. Dejus, D. R. Haeffner, and J. C. Lang, Performance of Advanced Photon Source Insertion Devices at High Photon Energies (50-300 keV), Rev. Sci. Instrum. 67 (9) (1996) CD-ROM

Shu, D., W. Yun, B. Lai, J. Barraza, and T. M. Kuzay, Double-Multilayer Monochromator Using a Modular Design for the Advanced Photon Source, Rev. Sci. Instrum. 66 (1995) 17861788

Shu, D., J. Barraza, C. Brite, T. Sanchez, and V. Tcheskidov, Beamline Standard Component Designs at the Advanced Photon Source, Rev. Sci. Instrum. 66 (1995) 1795-1797

Shu, D., Y. Li, D. Ryding, T. M. Kuzay, and D. Brasher, Explosion Bonding and its Applications in the Advanced Photon Source Front End and Beamline Component Designs, Rev. Sci. Instrum. 66 (1995) 1783-1785

Shu, D., V. Tcheskidov, T. Nian, D. R. Haeffner, E. E. Alp, D. Ryding, J. Collins, Y. Li, and T. M. Kuzay, Design of High Heat Load White-Beam Slits for Wiggler/Undulator Beamlines at the Advanced Photon Source, Rev. Sci. Instrum. 66 (1995) 1792-1794

Shu, D., C. Brite, T. Nian, W. Yun, D. R. Haeffner, E. E. Alp, D. Rygins, J. Collins, Y. Li, and T. M. Kuzay, Precision White-Beam Slit Design for the High Power Density X-ray Undulator Beamlines at the Advanced Photon Source, Rev. Sci. Instrum. 66 (1995) 17891791

Shu, D., and T. M. Kuzay, Design of the Commissioning Filter/Mask/Window Assembly for Undulator Beamline Front Ends at the Advanced Photon Source, Rev. Sci. Instrum. 67 (9) (1996) CD-ROM

Shu, D., and T. M. Kuzay, Smart X-ray Beam Position Monitor System for the Advanced Photon Source, Rev. Sci. Instrum. 67 (9) (1996) CD-ROM

Shu, D., D. Haeffner, and P. K. Job, White-Beam Transport Design for Insertion Device Beamlines at the Advanced Photon Source, Rev. Sci. Instrum. 67 (9) (1996) CD-ROM

Shu, D., J. Barraza, M. Ramanathan, J. Chang, and T. M. Kuzay, Alignment and Commissioning of the APS Beamine Front Ends, Rev. Sci. Instrum. 67 (9) (1996) CD-ROM

Shu, D., J. M. Minich, D. Haeffner, C. Brite, T. Nian, R. Dejus, and T. M. Kuzay, An Intelligent Filter Control System Using Fuzzy Logic for APS Insertion Device Beamlines, Rev. Sci. Instrum. 67 (9) (1996) CD-ROM 
Sinha, S. K., Diffuse Neutron Scattering from Surfaces and Interfaces, MRS Symposium Proceedings (1995) 376

Sinha, S. K., D. J. Lohse, and M. Y. Lin, Neutron Scattering as a Tool for Industrial Research, Physica B 213 and 214 (1995) 1

Sinha, S. K., J. W. Lynn, T. E. Grigereit, Z. Hossain, L. C. Gupta, R. Nagarajan, and C. Godart, Neutron Diffraction Study of Antiferromagnetic Order in the Magnetic Superconductor $\mathrm{ErNi}_{2} \mathrm{~B}_{2} \mathrm{C}$, Phys. Rev. B 51 (1995) 681

Sinha, S. K., M. Tolan, G. Vacca, Z. Li, M. Rafailovich, J. Sokolov, H. Lorenz, J. P. Kotthaus, Y. P. Feng, G. Grubel, and D. Abernathy, X-ray Scattering Studies of Molecular Ordering Phonomena in Confined Films, MRS Symposium Proc. 376 (1995) 175

Sinha, S. K., Surface Roughness by X-ray and Neutron Scattering Methods, Acta Physica Polonica A 89 (1996) 219

Sinha, S. K., X-ray Scattering Studies of Crystallization, Growth and Wetting Phenomena at Surfaces, Physica A 224 (1996) 140

Sinha, S. K., Surface Structure Reflectometry with X-rays, Current Opinion in Solid State and Materials Science 1 (1996) 645

Sinha, S. K., Y. P. Feng, C. A. Melendres, D. D. Lee, T. P. Russell, S. K. Satija, E. B. Sirota, and M. K. Sanyal, Off-Specular X-ray Scattering Studies of the Morphology of Thin Films, Physica A 231 (1996) 99-110

Smither, R. K., P. B. Fernandez, T. Graber, P. von Ballmoos, J. Naya, F. Albernhe, G. Vedrenne, and M. Faiz, Review of Crystal Diffraction and Its Application to Focusing Energetic Gamma Rays, Experimental Astronomy, volume 6.4 (1995) 47-56.

Smither, R. K., P. B. Fernandez, T. Graber, P. von Ballmoos, J. Naya, F. Albernhe, G. Vedrenne, and M. Faiz, Crystal Diffraction Lens Telescope for Focusing Nuclear Gamma Rays, in Gamma-Ray and Cosmic-Ray Detectors, Techniques, and Missions, SPIE 2806 (1996) 509-523

Song, J. J., Y. W. Kang, R. L. Kustom, B. Lai, D. C. Mancini, A. Nassiri, and V. White, Fabrication of mm-Wave Undulator/Linear Accelerator Cavities Using Deep X-ray Lithography, SPIE Proc. 2880 (1996) 288

Song, J. J., A. D. Feinerman Y. W. Kang, R. L. Kustom, B. Lai, A. Nassiri, V. White, and G. M. Well, Fabrication of mm-Wave Undulator Cavities Using Deep X-ray Lithography, Rev. Sci. Instrum. 67 (9) (1996) CD-ROM

Sözen, M., and T. M. Kuzay, Enhanced Heat Transfer in Round Tubes with Porous Inserts, Int. J. Heat and Fluid Flow 17 (1996) 124-129 
Sturhahn, W., T. S. Toellner, E. E. Alp, X. Zhang, M. Ando, Y. Yoda, S. Kikuta, M. Seto, C. W. Kimball, and B. Dabrowski, Phonon Density of States Measured by Inelastic Nuclear Resonant Scattering, Phys. Rev. Lett. 74 (1995) 3832-3835

Sturhahn, W., T. S. Toellner, K. W. Quast, R. Röhlsberger, and E. E. Alp, Inelastic Nuclear Resonant Scattering at the Advanced Photon Source, Rev. Sci. Instrum. 67 (9) (1996) CD-ROM

Sturhahn, W., T. S. Toellner, K. W. Quast, R. Röhlsberger, and E. E. Alp, Reduction of Detector Noise in Experiments with Synchrotron Radiation, Nucl. Instrum. Meth. Phys. Res. A 372 (1996) 455-457

Sturhahn, W., K. W. Quast, J. Metge, E. Gerdau, E. E. Alp, and T. S. Toellner, Electron Emission from ${ }^{57}$ Fe Nuclei Excited with Synchrotron Radiation, Phys. Rev. B 53 (1996) 171-175

Toellner, T. S., W. Sturhahn, R. Röhlsberger, E. E. Alp, C. H. Sowers, and E. E. Fullerton, Observation of Pure Nuclear Diffraction from a Fe/Cr Antiferromagnetic Multilayer, Phys. Rev. Lett. 74 (1995) 3475-3478

Tolan, M., G. Vacca, S. K. Sinha, Z. Li, M. Rafailovich, J. Sokolov, H. Lorenz, and J. P. Kotthaus, X-ray Diffraction from Microscopic Systems: Thin Films on Rough Surfaces, J. Phys. D: Appl. Phys. A231 (1995) 28

Tolan, M., G. Vacca, S. K. Sinha, Z. Li, M. H. Rafailovich, J. Sokolov, H. Lorenz, and J. P. Kotthaus, Si/Ge Films on Laterally Structured Surfaces: An X-ray Study of Conformal Roughness, Appl. Phys. Lett. 68 (1996) 191

Tolan, M., G. Vacca, J. Wang, S. K. Sinha, Z. Li, M. H. Rafailovich, J. Sokolov, A. Gibaud, and N. Lorenz, Thin Polymer Films on Rough Surfaces, Physica B 221 (1996) 53

Trakhtenberg, E. M., E. S. Gluskin, and S. Xu, The Vacuum System for Insertion Devices at the Advanced Photon Source, Rev. Sci. Instrum. 66 (1995) 1809-1811

Vakhrushev, S., A. Nabereznov, S. K. Sinha, Y. P. Feng, and T. Egami, Synchrotron X-ray Scattering Study of Lead Magnoniobate Relaxor Ferroelectric Crystals, J. Phys. Chem. Solids 57 (1996) 1517

Vasserman, I., and E. R. Moog, A Passive Scheme for ID End Correction, Rev. Sci. Instrum. 66 (1995) 1943-1945

Vinokurov, N. A., The Integral Equation for a High Gain FEL, Argonne National Laboratory Report ANL/APS/TB-27 (July 1996) 
von Ballmoos, P., J. E. Naya, F. Albernhe, G. Vedrenne, R. K. Smither, M. Faiz, P. Fernandez, and T. Graber, A Space Bourne Crystal Diffraction Telescope for the Energy Range of Nuclear Transitions, in Imaging in High Energy Astronomy, ed. L. Bassani and G. di Cocco, (Kluwer Academic Publishers, Dordrecht, The Netherlands, 1995) pp. 239245.

Wang, Z., and T. M. Kuzay, Variable Thickness Window: Thermal and Structural Analyses, Rev. Sci. Instrum. 66 (1995) 1821-1823

Wang, Z., and T. M. Kuzay, Thermal and Structural Analyses of Variable Thickness Plane Problems, PVP-Vol. 310, Fluid-Structure Interaction and Structural Mechanics (1995) $135-140$

Wang, Z., T. M. Kuzay, and L. Assoufid, Liquid-Metal, Pin-Fin Pressure Drop by Correlation in Cross Flow, Rev. Sci. Instrum. 66 (1995) 2260-2262

Wang, Z., T. M. Kuzay, T. Grace, and R. Dejus, X-ray Optical Analyses with X-ray Absorption Package (XRAP), Rev. Sci. Instrum. 66 (1995) 2263-2266

Wang, Z., W. Yun, T. M. Kuzay, G. Knapp, and S. Rogers, Thermal and Deformation Analyses of a Cryogenically Cooled Silicon Monochromator for High-Heat-Flux Synchrotron Sources, Rev. Sci. Instrum. 66 (1995) 2267-2269

Wang, Z., and U. Hahn, Design Analyses with XRAP for PETRA/HASYLAB Beamline Filters/Windows, in Optics for High-Brightness Synchrotron Radiation Beamlines II, SPIE Vol. 2856 (1996) 268-278

Wang, Z., and U. Hahn, Geometry and Parameter Optimization of PETRA Undulator Beamline Slits, in High Heat Flux Engineering III, SPIE Vol. 2855 (1996) 242-249

Wang, Z., and T. M. Kuzay, Interface of X-ray Absorption Package (XRAP) with ANSYS Code, in The Future of Simulation Tools: Computer Aided Engineering in the 21st Century, 1996 ANSYS Conf. Proc., pp. 3.261-3.267

Wang, Z., and T. M. Kuzay, On Filter Design and the Critical Thickness of Synchrotron X-ray Filters, Rev. Sci. Instrum. 67 (9) (1996) CD-ROM

Wang, Z., and T. M. Kuzay, Stress Level Evaluation of Thin Foils under Thermal Loading from a Brazing Process, PVP-Vol. 337, Fluid-Structure Interaction (eds. C. Y. Wang, D. C. Ma, Y. Y. Shin, R. F. Kulak, F. C. Chang, D. Brochard, F. J. Moody, and S. Kaneko, Book No. H01065 - 1996) 95-97

Wang, Z., and T. M. Kuzay, Using Metallic Filters in APS Undulator Beamlines, Rev. Sci. Instrum. 67 (9) (1996) CD-ROM 
Wang, Z., W. Yun, and T. M. Kuzay, A High-Precision Self-Adapted Optical Structure, Rev. Sci. Instrum. 67 (9) (1996) CD-ROM

Wang, Z., W. Yun, and T. M. Kuzay, Performance Analysis of a Novel Monochromator Design for the APS SRI-CAT Beamline 2-ID-E, Rev. Sci. Instrum. 67 (9) (1996) CD-ROM

Wu, X.Z., B. M. Ocko, H. Tang, E. B. Sirota, S. K. Sinha, and M. Deutsch, Surface Freezing in Binary Mixtures of Alkanes: New Phases and Phase Transitions, Phys. Rev. Lett. 75 (1995) 1332

Wu, X.Z., B. M. Ocko, H. Tang, E. B. Sirota, S. K. Sinha, and M. Deutsch, Surface Freezing in Binary Mixtures of Alkanes: New Phases and Phase Transitions, Physica B 221 (1996) 261

Xu, Z., I. McNulty, K. J. Randall, L. Yang, E. Gluskin, E. D. Johnson, and T. Oversluizen, X13A: A Versatile Soft X-ray Undulator Beamline, Rev. Sci. Instrum. 66 (1995) 18271829

Xu, Z., K. J. Randall, E. Gluskin, C.-C. Kao, E. D. Johnson, and C. T. Chen, X-ray Resonant Magnetic Scattering EllipsometerRev. Sci. Instrum. 67 (9) (1996) CD-ROM

Yahnke, C. J., G. Srajer, D. R. Haeffner, D. M. Mills, and L. Assoufid, Magnetic Compton Scattering Studies of the Invar Effect in $\mathrm{Fe}_{3} \mathrm{Pt}$, Mat. Res. Symp. Proc. 375 (1995) 221-227

Yang, L., McNulty, and E. Gluskin, An Intensity Interferometer for Soft X-rays, Rev. Sci. Instrum. 66 (1995) 2281-2283

Yao, S., P. J. Hesketh, and A. T. Macrander, Etching High Aspect Ratio (110) Silicon Grooves in CsOHJournal of the Electrochemical Society 142 (1995) L23-L25

Yoo, S. S., B. Rodricks, S. Sivananthan, J. P. Faurie, and P. A. Montano, MBE Grown CdTe Photoconductor Array Detector for X-ray Measurements, Rev. Sci. Instrum. 66 (1995) 2320-2322

Yun, W., B. Lai, K. J. Randall, S. Davey, D. R. Haeffner, P. K. Job, and D. Shu, Radiation Shielding of Insertion-Device Beamlines Using a Mirror as the First Optical Element, Argonne National Laboratory Report ANL/APS/TB-21 (1995)

Yun, W., Z. Cai, B. Lai, P. Ilinski, D. Legnini, and E. Gluskin, Photon Beam Diagnostics and Characterization Tools, Rev. Sci. Instrum. 67 (9) (1996) CD-ROM

Yun, W., A. Khounsary, B. Lai, K. J. Randall, I. McNulty, E. Gluskin, and D. Shu, Advantages of Using a Mirror as the First Optical Component for APS Undulator Beamlines, Rev. Sci. Instrum. 67 (9) (1996) CD-ROM 
Yun, W., B. Lai, D. Shu, A. Khounsary, Z. Cai, J. Barraza, and D. Legnini, Design of a Dedicated Beamline for X-ray Microfocusing- and Coherence-Based Techniques at the Advanced Photon Source, Rev. Sci. Instrum. 67 (9) (1996) CD-ROM 
APPENDIX 1 


\section{Patents Obtained by XFD Staff}

Alp, E.E., T.M. Mooney, and T. Toellner, High Energy Resolution, High Angular Acceptance Crystal Monochromator, U.S. Patent No. 5,524,040, granted June 1996

Khounsary, A., Inclined Monochromator for High Heat-Load Synchrotron X-Ray Radiation, U.S. Patent No. 5,287,795, granted February 1994

Kuzay, T., and D. Shu, Photon Beam Position Monitor, U.S. Patent No. 5,387,795, granted February 1995

Kuzay, T., Process of Making Cryogenically Cooled High Thermal Performance Crystal Optics, U.S. Patent No. 5,123,982, granted June 1992

Shu, D., and T. Kuzay, Integral Window/Photon Beam Position Monitor and Beam Flux Detectors for X-Ray Beams, U.S. Patent No. 5,404,014, granted April 1995

Shu, D., and J. Barraza, Low-Profile, High-Load, Vertical Rolling Positioning Stage, U.S. Patent No. 5,526,903, granted June 1996

Smither, R.K., Electromagnetic Induction Pump for Pumping Liquid Metals and other Conductive Liquids, U.S. Patent No. 5,209,646, granted May 1993 


\section{XFD Primary Web Pages}

The starting WEB pages for major categories are:

XFD Home Page - Starting point for all XFD-related activities

http://www.aps.anl.gov/xfd/WWW/xfd/welcome.html

User Contact Information - All user-related information is available here

http://www.aps.anl.gov/xfd/WWW/xfd/communicator/useroffice/homepage.html

APS Operations Information Home Page - Starting point for all operations-related information http://www.aps.anl.gov/xfd/WWW/xfd/operations/welcome.html

APS Collaborative Access Team (CAT) Home Pages

http://www.aps.anl.gov/cats/cathome.html

SRI-CAT Home Page

http://www.aps.anl.gov/xfd/WWW/xfd/sri_cat/sri_cat_home.html

Operations-related additonal key information:

APS Operations Schedules

http://www.aps.anl.gov/xfd/W/WW/xfd/calendar/calendar.html

Storage Ring Status Information http://www.aps.anl.gov/xfd/WWW/xfd/blops/srStatus.cgi

Status Information on Important Technical Systems

http://www.aps.anl.gov/asd/operations/techStatus.html

Operating Envelop for Each Beamline

http://www.aps.anl.gov/xfd/WWW/xfd/operations/approved/ops.html

Beamline Station Operating Status http://www.aps.anl.gov/xfd/wWw/xfd/operations/bin/station.cgi

APS Beamline Experiment Safety Forms Home Page http://www.aps.anl.gov/xfd/wWw/xfd/expsafety/welcome.html

Quick Overview of Installation and Commissioning Status of IDs, FE, and Beamlines http://www.aps.anl.gov/xfd/WWW/xfd/blops/BL_Installation.html

APS user-related additonal key information:

CAT Chat Minutes

http://www.aps.anl.gov/xfd/WWW/xfd/cat_chats/CAT_Chat_ToC.html

CAT Communicator

http://www.aps.anl.gov/xfd/WWW/xfd/communicator/communicator.html

List of APS Technical Bulletins

http://www.aps.anl.gov/xfd/wWW/xfd/tech_bulletins/List_of_TBs.html

Workshop Reports and Conference Proceedings

http://www.aps.anl.gov/xfd/WWW/xfd/tech_bulletins/Workshop_Report_List.html

XFD Safety and Environmental Protection Policies and Practices

http://www.aps.anl.gov/xfd/WWW/xfd/esh/esh_home.html

XFD Technical Procedures

http://www.aps.anl.gov/xfd/WWW/xfd/safety/safety_index.html 
Software-related additonal key information:

EPICS Software Distribution

http://www.aps.anl.gov/xfd/WWW/xfd/SoftDist/Welcome.html

APS Design Exchange - Repository for all beamline drawings

http://dxchange.aps1.anl.gov 


\section{Review Status of APS Collaborative Access Teams as of January 21, 1997}

\begin{tabular}{|c|c|c|c|c|c|c|c|c|c|}
\hline \multirow{2}{*}{$\begin{array}{c}\text { CAT } \\
\text { Acronym }\end{array}$} & \multirow{2}{*}{$\begin{array}{c}\text { CAT } \\
\text { Director }\end{array}$} & \multirow{2}{*}{$\begin{array}{c}\text { Sector } \\
\text { Assignment }\end{array}$} & \multicolumn{2}{|c|}{ Letter of Intent } & \multicolumn{2}{|c|}{ Proposal } & \multicolumn{2}{|c|}{ Management Plan } & \multirow{2}{*}{$\begin{array}{l}\text { Funding } \\
\text { Letters } \\
\text { Received }\end{array}$} \\
\hline & & & Submitted & Accepted & Submitted & Accepted & Submitted & Accepted & \\
\hline BESSRC & $\begin{array}{l}\text { Pedro } \\
\text { Montano }\end{array}$ & 11,12 & $5 / 1 / 90$ & $6 / 12 / 90$ & $\begin{array}{c}3 / 15 / 91 \\
3 / 31 / 93^{\star}\end{array}$ & $\begin{array}{c}11 / 1 / 91 \\
5 / 14 / 93^{*}\end{array}$ & $3 / 21 / 94$ & $7 / 5 / 94$ & Received \\
\hline $\mathrm{BIO}$ & Grant Bunker & 18 & $5 / 1 / 90$ & $6 / 12 / 90$ & $3 / 15 / 91$ & $11 / 1 / 91$ & $11 / 14 / 95$ & $1 / 3 / 96$ & $12 / 1 / 95$ \\
\hline CARS & $\begin{array}{l}\text { J. Keith } \\
\text { Moffat }\end{array}$ & $13,14,15$ & $\begin{array}{r}5 / 1 / 90 \\
1 / 15 / 92\end{array}$ & $\begin{array}{l}6 / 12 / 90 \\
3 / 31 / 92\end{array}$ & $\begin{array}{l}3 / 15 / 91 \\
3 / 31 / 93 \\
8 / 13 / 93\end{array}$ & $\begin{array}{l}11 / 1 / 91 \\
5 / 14 / 93 \\
9 / 30 / 93\end{array}$ & $11 / 22 / 93$ & $3 / 14 / 94$ & $\begin{array}{c}4 / 5 / 94 \\
9 / 19 / 96\end{array}$ \\
\hline$C M C$ & L. Doon Gibbs & 9 & $5 / 1 / 90$ & $6 / 12 / 90$ & $3 / 15 / 91$ & $11 / 1 / 91$ & $5 / 26 / 94$ & $10 / 28 / 94$ & $3 / 9 / 95$ \\
\hline DND & $\begin{array}{l}\text { Panayotis } \\
\text { Georgopoulos }\end{array}$ & 5 & $\begin{array}{c}5 / 1 / 90 \\
12 / 15 / 91\end{array}$ & $\begin{array}{l}6 / 12 / 90 \\
1 / 30 / 92\end{array}$ & $3 / 15 / 91$ & $4 / 1 / 92$ & $1 / 6 / 93$ & $6 / 16 / 93$ & $8 / 3 / 93$ \\
\hline IMCA & $\begin{array}{l}\text { Andrew } \\
\text { Howard }\end{array}$ & 17 & $5 / 1 / 90$ & $6 / 12 / 90$ & $3 / 15 / 91$ & $11 / 1 / 91$ & $2 / 20 / 94$ & $5 / 24 / 94$ & $12 / 15 / 93$ \\
\hline IMM & $\begin{array}{l}\text { Simon } \\
\text { Mochrie }\end{array}$ & 8 & $5 / 1 / 90$ & $6 / 12 / 90$ & $3 / 15 / 91$ & $11 / 1 / 91$ & $2 / 22 / 94$ & $4 / 8 / 94$ & $\begin{array}{l}2 / 22 / 94 \\
4 / 18 / 94\end{array}$ \\
\hline MHATT & Walter Lowe & 7 & $5 / 1 / 90$ & $6 / 12 / 90$ & $3 / 15 / 91$ & $11 / 1 / 91$ & $11 / 29 / 93$ & $5 / 25 / 94$ & $6 / 1 / 94$ \\
\hline$M R$ & Bruce Bunker & 10 & $5 / 1 / 90$ & $6 / 12 / 90$ & $3 / 15 / 91$ & $4 / 1 / 92$ & $7 / 19 / 94$ & $11 / 21 / 94$ & $11 / 28 / 94$ \\
\hline$\mu$ & Alan Goldman & 6 & $5 / 1 / 90$ & $6 / 12 / 90$ & $3 / 15 / 91$ & $4 / 1 / 92$ & $6 / 6 / 95$ & $6 / 20 / 96$ & $\begin{array}{c}6 / 5 / 96 \\
6 / 26 / 96\end{array}$ \\
\hline PNC & Edward Stern & 20 & $5 / 1 / 90$ & $6 / 12 / 90$ & $3 / 15 / 91$ & $4 / 1 / 92$ & $6 / 6 / 94$ & $6 / 28 / 94$ & $12 / 16 / 94$ \\
\hline$S B C$ & $\begin{array}{l}\text { Edwin } \\
\text { Westbrook }\end{array}$ & 19 & $5 / 1 / 90$ & $6 / 12 / 90$ & $3 / 15 / 91$ & $4 / 1 / 92$ & $12 / 22 / 93$ & $3 / 14 / 94$ & $3 / 94$ \\
\hline SAI & Dennis Mills & $1,2,3$ & $5 / 1 / 90$ & $6 / 12 / 90$ & $3 / 15 / 91$ & $3 / 31 / 92$ & $12 / 22 / 93$ & $2 / 16 / 94$ & Received \\
\hline UNI & Haydn Chen & 33,34 & $5 / 1 / 90$ & $6 / 12 / 90$ & $\begin{array}{c}3 / 15 / 91 \\
\text { (Sector 33) } \\
4 / 4 / 96 \\
\text { (Sector 34) }\end{array}$ & $\begin{array}{c}11 / 1 / 91 \\
3 / 97\end{array}$ & $7 / 21 / 93$ & $8 / 23 / 93$ & $4 / 21 / 94$ \\
\hline
\end{tabular}

${ }^{*}$ Addendum (scientific justification for 4.1-cm-period undulator). 


\begin{tabular}{|c|c|c|c|c|c|c|c|c|c|}
\hline \multirow{2}{*}{$\begin{array}{c}\text { CAT } \\
\text { Acronym }\end{array}$} & \multicolumn{2}{|c|}{ CDR } & \multirow{2}{*}{$\begin{array}{c}\text { MOU } \\
\text { Signed }\end{array}$} & \multicolumn{2}{|c|}{$\begin{array}{c}\text { Preliminary } \\
\text { Design Report } \\
\end{array}$} & \multicolumn{2}{|c|}{ Final Design Report } & \multicolumn{2}{|c|}{ Safety Plan } \\
\hline & Submitted & Approved & & Submitted & Approved & Submitted & Approved & Submitted & Approved \\
\hline BESSRC & $11 / 1 / 92$ & $7 / 28 / 93$ & $7 / 6 / 94$ & $2 / 17 / 95$ & $5 / 22 / 95$ & $\begin{array}{c}10 / 31 / 95 \\
(12 \mathrm{BM}) \\
4 / 18 / 96 \\
(12 \mathrm{ID})\end{array}$ & $\begin{array}{c}7 / 23 / 96 \\
(12 B M \\
\text { and } \\
121 D)\end{array}$ & $3 / 15 / 96$ & $6 / 6 / 96$ \\
\hline$B I O$ & $1 / 94$ & $5 / 10 / 94$ & $12 / 4 / 95$ & $7 / 23 / 96$ & $12 / 20 / 96$ & & & $6 / 19 / 96$ & $8 / 29 / 96$ \\
\hline CARS & $\begin{array}{c}11 / 1 / 92 \\
\text { (2 sectors) } \\
10 / 1 / 93 \\
\text { (3rd sec.) }\end{array}$ & $\begin{array}{l}1 / 14 / 93 \\
1 / 13 / 94\end{array}$ & $\begin{array}{c}4 / 27 / 94 \\
(\text { sec. } 13,14) \\
10 / 21 / 96 \\
\text { (sec. } 15)\end{array}$ & $\begin{array}{l}11 / 4 / 94 \\
\text { (sec. 14) } \\
12 / 12 / 95 \\
\text { (sec. 13) }\end{array}$ & $\begin{array}{l}12 / 15 / 94 \\
5 / 21 / 96\end{array}$ & $\begin{array}{l}7 / 17 / 95 \\
\text { (sec. 14) } \\
10 / 21 / 96 \\
\text { (sec. 13) }\end{array}$ & $12 / 12 / 95$ & $5 / 1 / 96$ & $6 / 12 / 96$ \\
\hline$C M C$ & $8 / 14 / 92$ & $1 / 7 / 93$ & $10 / 31 / 94$ & $9 / 20 / 95$ & $2 / 7 / 96$ & & & $5 / 26 / 94$ & $10 / 28 / 94$ \\
\hline$D N D$ & $11 / 1 / 92$ & $7 / 28 / 93$ & $12 / 15 / 93$ & $12 / 5 / 94$ & $4 / 19 / 94$ & $7 / 19 / 95$ & $11 / 3 / 95$ & $11 / 11 / 95$ & $5 / 28 / 96$ \\
\hline$M C A$ & $11 / 1 / 92$ & $7 / 28 / 93$ & $9 / 16 / 94$ & $10 / 20 / 95$ & $1 / 30 / 96$ & & & $5 / 8 / 96$ & $7 / 25 / 96$ \\
\hline$I M M$ & $10 / 2 / 93$ & $1 / 13 / 94$ & $5 / 19 / 94$ & $8 / 2 / 95$ & $1 / 10 / 96$ & $7 / 15 / 96$ & $10 / 96$ & $5 / 30 / 96$ & $8 / 15 / 96$ \\
\hline MHATT & $11 / 1 / 92$ & $1 / 7 / 93$ & $6 / 2 / 94$ & $2 / 6 / 96$ & $7 / 23 / 96$ & & & $11 / 29 / 93$ & $5 / 25 / 94$ \\
\hline$M R$ & $11 / 92$ & $7 / 28 / 93$ & $5 / 24 / 95$ & $2 / 8 / 96$ & $7 / 23 / 96$ & & & $5 / 23 / 96$ & $7 / 19 / 96$ \\
\hline$\mu$ & $11 / 92$ & $1 / 14 / 93$ & $8 / 27 / 96$ & $5 / 1 / 96$ & & & & $6 / 9 / 95$ & $6 / 20 / 96$ \\
\hline PNC & $8 / 7 / 92$ & $1 / 14 / 93$ & $5 / 11 / 95$ & $6 / 14 / 95$ & $9 / 19 / 95$ & $12 / 19 / 96$ & & $8 / 14 / 96$ & $10 / 7 / 96$ \\
\hline$S B C$ & $11 / 1 / 92$ & $7 / 28 / 93$ & $3 / 17 / 94$ & $6 / 22 / 95$ & $9 / 22 / 95$ & $\begin{array}{l}2 / 9 / 96 \\
19-I D\end{array}$ & $7 / 8 / 96$ & $1 / 23 / 96$ & $3 / 12 / 96$ \\
\hline SRI & $11 / 1 / 92$ & $1 / 14 / 93$ & $4 / 29 / 94$ & $7 / 25 / 94$ & $10 / 15 / 94$ & \begin{tabular}{|c|}
$2 / 2 / 95$ \\
(sector 1) \\
$3 / 8 / 95$ \\
(sector 3 ) \\
$9 / 20 / 95$ \\
(sector 2)
\end{tabular} & $\begin{array}{c}/ 24 / 95 \\
(\text { sec. 1) } \\
3 / 27 / 95 \\
(\text { sec. 3) } \\
12 / 95 \\
\text { (sec. 2) }\end{array}$ & $2 / 2 / 95$ & $5 / 17 / 95$ \\
\hline UNI & $8 / 92$ & $1 / 14 / 93$ & $4 / 27 / 94$ & $\begin{array}{c}9 / 6 / 94 \\
\text { (sec. 33 ID) } \\
1 / 20 / 97 \\
\text { (sec. 33 BM) }\end{array}$ & $10 / 21 / 94$ & $4 / 20 / 95$ & $8 / 7 / 95$ & $2 / 12 / 96$ & $8 / 30 / 96$ \\
\hline
\end{tabular}




\section{Bibliography of Publications Produced for APS Users}

\section{CAT Communicator}

CAT Communicator, Vol. I, No. 1, September 1990

CAT Communicator, Vol. I, No. 2, November 1990

CAT Communicator, Vol. II, No. 1, February 1991

CAT Communicator, Vol. II, No. 2, October 1991

CAT Communicator, Vol. III, No. 1, January 1992

CAT Communicator, Vol. III, No. 2, February 1992

CAT Communicator, Vol. III, No. 3, May 1992

CAT Communicator, Vol. III, No. 4, September 1992

CAT Communicator, Vol. IV, No. 1, January 1993

CAT Communicator, Vol. IV, No. 2, April 1993

CAT Communicator, Vol. IV, No. 3, October 1993

CAT Communicator, "Special Edition," December 15, 1993

CAT Communicator, Vol. V, No. 1, April 1994

CAT Communicator, Vol. V, No. 2, November 1994

CAT Communicator, Vol. VI, No. 1, March 1995

CAT Communicator, Vol. VI, No. 2, July 1995

CAT Communicator, Vol. VI, No. 3, October 1995

CAT Communicator, Vol. VII, No. 1 (in preparation)

\section{Conference Proceedings}

Proceedings of the First Users Meeting for the Advanced Photon Source, ANL/APS-CP-1 (February 1988)

Proceedings of the Second Users Meeting for the Advanced Photon Source, ANL/APS-CP-2

(November 1988)

Proceedings of the Third Users Meeting for the Advanced Photon Source, ANL/APS-CP-3

(June 1990)

Proceedings of the Conference on Advanced X-ray/EUV Radiation Sources and Applications,

Eds. J.P. Knauer and G.K. Shenoy, SPIE Vol. 1345 (1990)

Proceedings of the Fourth Users Meeting for the Advanced Photon Source, ANL/APS-CP-4

(February 1992)

Proceedings of the Conference on High Heat Flux Engineering, Ed. A.M. Khounsary, SPIE

Vol. 1739 (1992)

Proceedings of the Fifth Users Meeting for the Advanced Photon Source, ANL/APS-CP-5

(December 1992)

Proceedings of the Conference on High Heat Flux Engineering II, Ed. A.M. Khounsary, SPIE

Vol. 1997 (1993) 
Proceedings of the Sixth Users Meeting for the Advanced Photon Source, ANL/APS-CP-6

(December 1994)

Proceedings of the Conference on Radiation Instrumentation (SRI-95), Eds. G.K. Shenoy and

J. Dehmer, CD ROM Supplement to the Review of Scientific Instruments (American

Insitute of Physics, 1996)

\section{Workshop Reports}

X-Ray Synchrotrons and the Development of New Materials: Workshop Report, ANL/APS-TM-1 (February 1988)

Time-Resolved Studies and Ultrafast Detectors: Workshop Report, ANL/APS-TM-2

(February 1988)

Synchrotron X-Ray Sources and New Opportunities in Earth Sciences: Workshop Report, ANL/APS-TM-3 (April 1988)

Chemical Applications of Synchrotron Radiation: Workshop Report, ANL/APS-TM-4 (April 1989)

Workshop on High Heat Load X-Ray Optics, ANL/APS-TM-6 (January 1990)

Synchrotron X-Ray Sources and New Opportunities in the Soil and Environmental Sciences:

Workshop Report, ANL/APS-TM-7 (July 1990)

Atomic Physics at the Advanced Photon Source: Workshop Report, ANL/APS-TM-8

(October 1990)

Applications of Synchrotron Radiation to Chemical Engineering Science, ANL/APS-TM-9

(July 1991)

Working Group Summary Reports from the Advanced Photon Source Reliability Workshop, ANL/APS-TM-10 (May 1992)

Poster Session: Fifth Users Meeting for the Advanced Photon Source, ANL/APS-TM-11

(November 1992)

1993 CAT Workshop on Beamline Optical Designs, ANL/APS-TM-12 (November 1993)

International Workshop on Magnetic Measurements of Insertion Devices, ANL/APS-TM-13

(October 1993)

Atomic Physics at High Brilliance Synchrotron Sources, ANL/APS-TM-14 (August 1994)

Detectors for Third-Generation Synchrotron Sources: Workshop Report, ANL/APS-TM-15

(December 1994)

Atomic Physics with Hard X-rays from High Brilliance Synchrotron Light Sources,

ANL/APS/TM-16 (August 1996)

\section{Technical Bulletins}

Characteristics of the 7-GeV Advanced Photon Source: A Guide for Users, G.K. Shenoy, P.J. Viccaro, and D.M. Mills, ANL-88-9 (February 1988)

APS Undulator and Wiggler Sources: Monte-Carlo Simulation, S. L. Xu, B. Lai, and

P. J. Viccaro, ANL/APS/TB-1 (February 1992) 
Use of a Mirror as the First Optical Component for an Undulator Beamline at the APS,

W. Yun, A. Khounsary, B. Lai, and E. Gluskin, ANL/APS/TB-2 (September 1992)

Undulator A Characteristics and Specifications, B. Lai, A. Khounsary, R. Savoy, L. Moog, and E. Gluskin, ANL/APS/TB-3 (February 1993)

APS High Heat Load Monochromator, W.-K. Lee and D. M. Mills, ANL/APS/TB-4

(February 1993)

Functional Description of APS Beamline Front Ends, T. M. Kuzay, ANL/APS/TB-5

(February 1993)

APS X-Ray Optics Fabrication and Characterization Facility, S. Davey, ANL/APS/TB-6

(February 1993)

Guide to Beamline Radiation Shielding Design at the Advanced Photon Source, N. Ipe,

D. R. Haeffner, E. E. Alp, S. C. Davey, R. J. Dejus, U. Hahn, B. Lai, K. J. Randall, and

D. Shu, ANL/APS/TB-7 (November 1993)

CAT Questions and Answers, ANL/APS/TB-8 (February 1993)

APS Sector Layout: Utilities, Etc., S. Davey, ANL/APS/TB-9 (February 1993)

Wiggler A Characteristics and Specifications, B. Lai, A. Khounsary, and E. Gluskin,

ANL/APS/TB-11 (February 1993)

The Insertion Device Magnetic Measurement Facility: Prototype and Operational Procedures,

L. Burkel, R. Dejus, J. Maines, J. O’Brien, J. Pflüger, and I. Vasserman, ANL/APS/TB-12

(March 1993)

Report on the Value Engineering Workshop on APS Beamline Front Ends, T. M. Kuzay,

ANL/APS/TB-13 (January 1993)

APS Beamline Design and Construction Requirements: A Reference Manual for Designers and

Builders, version 1.0, ANL/APS/TB-14 (May 1994)

APS User Guide: What you.need to work at the APS, version 2.0, ANL/APS/TB-15

(October 1995)

Advanced Photon Source Accelerator Utrahigh Vacuum Guide, C. Liu and J. Noonan, ANL/APS/TB-16 (March 1994)

Undulator A Characteristics and Specifications: Enhanced Capabilities, R. Dejus, B. Lai,

E. Moog, and E. Gluskin, ANL/APS/TB-17 (May 1994)

The Cryogenic Cooling Program at the Advanced Photon Source, C. S. Rogers, D. M. Mills, and

L. Assoufid, ANL/APS/TB-18 (June 1994)

A Finite Element Analysis of Room Temperature Silicon Crystals for the APS Bending-Magnet

and Insertion-Device Sources, L. Assoufid, W.-K. Lee, and D. M. Mills, ANL/APS/TB-19

(August 1994)

Bremsstrahlung Scattering Calculations for the Beam Stops and Collimators in the APS

Insertion-Device Beamlines, P. K. Job, D. R. Haeffner, and D. Shu, ANL/APS/TB-20

(December 1994)

Radiation Shielding of Insertion-Device Beamlines Using a Mirror as the First Optical Element,

W. Yun, B. Lai, K. J. Randall, S. Davey, D. R. Haeffner, P. K. Job, and D. Shu,

ANL/APS/TB-21 (February 1995)

Magnetic Measurements of the Elliptical Multipole Wiggler Prototype, D. Frachon, I. Vasserman,

P. M. Ivanov, E. A. Medvedko, E. Gluskin, and N. A. Vinokurov, ANL/APS/TB-22

(March 1995)

APS User Safety Guide: A reference for work at the APS, ANL/APS/TB-23 (October 1995) 
Diamond Monochromators for APS Undulator-A Beamlines, R. C. Blasdell, L. A. Assoufid, and D. M. Mills, ANL/APS/TB-24 (September 1995)

The Advance Photon Source - A National Synchrotron Radiation Research Facility at Argonne, ANL/APS/TB-25 (October 1995)

The Advanced Photon Source List of Parameters, compiled by Hana M. Bizek, ANL/APS/TB-26 (July 1996)

The Integral Equation for a High Gain FEL, N. A. Vinokurov, ANL/APS/TB-27 (July 1996) 


\section{Conferences and Workshops Held at the APS since 1990}

Workshop on Synchrotron X-ray Sources and New Opportunities in the Agricultural and Related Sciences; January 8-10, 1990; Argonne National Laboratory

Anomalous Phasing Techniques in Macromolecular Crystallography; February 26-27, 1990; University of Alabama at Birmingham

Workshop on Atomic Physics at the APS; March 29-30, 1990; Argonne National Laboratory

APS Workshop for Prospective CAT Directors and Institutional Representatives; October 17-18, 1990; Argonne National Laboratory (approximately 100 participants)

Workshop on Front End Engineering of Synchrotron Light Sources; February 18-19, 1991; Argonne National Laboratory

Workshop on the Applications of Synchrotron Radiation to Chemical Engineering Science; April 22-23, 1991; Argonne National Laboratory (approximately 85 participants)

Fourth Users Meeting for the Advanced Photon Source; May 7-8, 1991; Argonne National Laboratory (approximately 200 participants)

Workshop for Prospective APS CATs; January 22-23, 1992; Argonne National Laboratory (approximately 130 participants)

CAT Scientific Programs Workshop; July 14-15, 1992; Argonne National Laboratory (approximately 140 participants)

CAT Safety Planning Workshop; July 16, 1992; Argonne National Laboratory (approximately 30 participants)

Fifth Users Meeting for the Advanced Photon Source; October 14-15, 1992; Argonne National Laboratory (approximately 340 participants)

1993 CAT Workshop; February 25-26, 1993; Argonne National Laboratory (approximately 150 participants)

CAT Beamline Controls Workshop; March 16-18, 1994; Hickory Ridge Conference Center (approximately 50 participants)

Sixth Users Meeting for the Advanced Photon Source; May 25-26, 1994; Argonne National Laboratory (approximately 500 participants)

Three mini-workshops (Coherent X-ray Scattering, Microfocusing Technology and Applications, and Polarization); May 26, 1994; Argonne National Laboratory (approximately 250 participants)

CAT Procurement Information Meeting; September 12, 1994; Argonne National Laboratory (approximately 20 participants)

APS User Agreement Information Meeting; February 7, 1995; Argonne National Laboratory (approximately 20 participants) 
APS Beamline Commissioning Workshop; May 16, 1995; Argonne National Laboratory (approximately 65 participants)

APS X-ray Centennial Symposium; October 16, 1995; Argonne National Laboratory (approximately 600 participants)

Fourteen mini-workshops on synchrotron radiation applications and instrumentation; October 17 20, 1995; Argonne National Laboratory (approximately 800 participants)

Seventh Users Meeting for the Advanced Photon Source; October 18, 1995; Argonne National Laboratory (approximately 800 participants)

Synchrotron Radiation Instrumentation '95; October 18-20, 1995; Argonne National Laboratory (approximately 700 participants)

IUCr Synchrotron Radiation Satellite Meeting; August 4-7, 1996; Argonne National Laboratory (approximately 250 participants) 


\section{Outline of the Current APS User Policies and Procedures (3/97)}

\section{INTRODUCTION}

1.1. Advanced Photon Source Mission Statement

1.2. User Safety Policy

1.3. About This Manual

2. KEY TERMS AND ABBREVIATIONS

3. THE ROLE OF APS STAFF

3.1. Overview

3.2. APS Organization

3.3. Conventional Facilities and Utilities Supplied by APS

3.4. Technical Components Supplied and Installed by APS

3.5. Standardization and Modularization of Other Beamline Components

3.6. Design Exchange

3.7. Radiation Shielding Guidelines

3.8. Design Reviews

3.9. X-ray Optics Fabrication and Characterization Facility

3.10. Assistance with Installation of User-Supplied Components

3.11. APS Site Safety Training

3.12. Guidelines for CAT Safety Programs

3.13. Safety Reviews

3.14. Safety of User Operations

3.15. General Operational Support

3.16. Other User Services

3.17. Collaborative Research Program

3.18. Technical Workshops and Bulletins

3.19. General Communication with the User Community

3.20. SRI-CAT

3.21. APS Contact Information

\section{PARTICIPATION BY COLLABORATIVE ACCESS TEAMS}

4.1. Overview of CAT Responsibilities

4.2. Forming a CAT

4.3. Allocation of Sectors to CATs

4.4. User Agreements

4.5. Site Access, Orientation, and Sector-specific Safety Training 
4.6. User Accounts

4.7. Technical/Safety Reviews by APS

4.8. CAT Safety Oversight Committees

4.9. Vacuum Protection

4.10. Ozone Mitigation

4.11. Management of Sealed Sources

4.12. Incidental Crane Operator Training

4.13. Use of Third-Party Contractors

4.14. Tricycles

4.15. Visitors

4.16. Procurement of Major Beamline Components

4.17. Obtaining APS/ANL Support Services

4.18. Intra-Facility Communications

4.19. Scheduling of Beam Time

4.20. Special Considerations for CAT Members Conducting Proprietary Research

4.21. Written Reports

4.22. Reviews by the Proposal Evaluation Board

4.23. Decommissioning of Beamlines and Removal of CAT-Owned Property

\section{PARTICIPATION BY INDEPENDENT INVESTIGATORS}

5.1. Access Policy

5.2. Initiation of II Programs by CATs

5.3. Proposal Submission and Review

5.4. Scheduling of Beam Time

5.5. Facilities, Equipment, and Technical Support Provided by the Host CAT

5.6. Safety Training And Reviews Provided by the Host CAT

5.7. Tracking of II Participation

5.8. Special Considerations for IIs Conducting Proprietary Research

5.9. User Agreements

5.10. Site Access, Orientation, and Sector-specific Safety Training

5.11. User Accounts

5.12. Obtaining APS/ANL Support Services

5.13. Safety Practices

5.14. Intra-facility Communications

5.15. Written Reports

\section{COMMITTEES AND THEIR ROLES}

6.1. APSUO Steering Committee

6.2. Research Directorate

6.3. Proposal Evaluation Board

6.4. Scientific Review Panels

6.5. Instrumentation Feasibility Study Panel 
6.6. Management Plan Review Group

6.7. Beamline Standardization and Modularization Committee

6.8. APS CAT Safety Committee

7. MAKING CHANGES IN THE APS USER POLICIES AND PROCEDURES

LIST OF APPENDIXES 


\section{Metrology Results of Mirrors Delivered and Characterized to Date}

$\Delta \mathrm{a}$ is the rms slope error, $\mathrm{s}$ is the rms roughness, and $\mathrm{R}$ is the radius of curvature.

\begin{tabular}{|c|c|c|c|c|c|c|c|c|c|c|c|c|}
\hline $\begin{array}{l}19-B M \\
\text { SBC }\end{array}$ & Rockwell & 1.02 & $\begin{array}{l}\text { Flat } \\
\text { bendable }\end{array}$ & 1.0 & 2.0 & $600.0-\infty$ & 0.9 & 2.0 & - & 1.0 & 2.3 & $\begin{array}{l}\text { Measured } \\
\text { flat }\end{array}$ \\
\hline $\begin{array}{l}2 \text { - ID } \\
\text { SRI }\end{array}$ & Rockwell & 1.20 & Flat & $<4.0$ & 4.0 & $\infty$ & $2.2 \%$ & 2.1 & - & 2.2 & 2.7 & - \\
\hline $\begin{array}{l}2 \text { - ID-C } \\
\text { SRI }\end{array}$ & $\begin{array}{l}\text { Continental } \\
\text { Optical }\end{array}$ & 0.24 & Spherical & 1.0 & 5.0 & $448.7 \pm 1 \%$ & $\begin{array}{l}\text { Not } \\
\text { Avail. }\end{array}$ & $\begin{array}{l}\text { Not } \\
\text { Avail. }\end{array}$ & - & 0.8 & $<2.0$ & 448.2 \\
\hline $\begin{array}{l}\text { 2-ID-C } \\
\text { SRI }\end{array}$ & $\begin{array}{l}\text { Continental } \\
\text { Optical }\end{array}$ & 0.18 & Spherical & 1.0 & 5.0 & $171.9 \pm 1 \%$ & $\begin{array}{l}\text { Not } \\
\text { Avail. }\end{array}$ & $\begin{array}{l}\text { Not } \\
\text { Avail. }\end{array}$ & - & 1.1 & 2.0 & 172.4 \\
\hline $\begin{array}{l}1-\mathrm{BM} \\
\text { SRI }\end{array}$ & ZEISS Corp. & 1.20 & $\begin{array}{l}\text { Flat } \\
\text { bendable }\end{array}$ & $<5.0$ & $<3.0$ & $1200.0-\infty$ & 2.4 & 2.6 & $\begin{array}{c}1200.0 \\
-\infty\end{array}$ & 4.0 & 2.8 & $1200.0-\infty$ \\
\hline $\begin{array}{l}5-\mathrm{BM}^{*} \\
\text { DND }\end{array}$ & PSI & 1.50 & $\begin{array}{l}\text { Flat } \\
\text { bendable }\end{array}$ & - & - & - & $\begin{array}{l}\text { Not } \\
\text { Avail. }\end{array}$ & $\begin{array}{l}\text { Not } \\
\text { Avail. }\end{array}$ & $\begin{array}{l}\text { Not } \\
\text { Avail. }\end{array}$ & - & 10.0 & - \\
\hline
\end{tabular}

*This mirror has not received the final coating yet. 


\section{Invited and Colloquium Talks by XFD Staff}

Alp, E.E., Nuclear Resonant Scattering Studies at the Advanced Photon Source, Materials Research Society Spring Meeting, San Fransisco, CA (1996).

Alp, E.E., Nuclear Resonant Scattering of Synchrotron Radiation, Physics Department Seminar, Purdue University (1996).

Alp, E.E., High Energy Resolution Inelastic X-Ray Scattering, Theory Workshop on Applications of Synchrotron Radiation in Materials Science, Argonne National Laboratory (1996).

Alp, E.E., Inelastic Nuclear Resonant Scattering of Synchrotron Radiation, US-Mexico Workshop on Applications of Synchrotron Radiation, Argonne National Laboratory (1996).

Alp, E.E., Use of Synchrotron Radiation in Mossbauer Spectroscopy: Recent Developments, Latin American Conference on Applications of Mossbauer Effect, Cuzco, Peru (1996).

Alp, E.E., Nuclear Resonant Scattering Studies with Synchrotron Radiation, Physics Department Colloquim, University of Missouri (1996).

Alp, E.E., Inelastic Nuclear Resonant Scattering with sub-meV Resolution, Materials Research Society Fall Meeting, Boston, MA (1996).

Alp, E.E., Recent Developments in Energy and Time Domain Mossbauer Spectroscopy with Synchrotron Radiation, Institut for Nuclear Solid State Physics, Katholike University of Leuven, Belgium (1997).

Alp, E.E., Inelastic Nuclear Resonant Scattering with sub-meV Resolution, American Physical Society March Meeting, Kansas City, MO (1997).

Banerjee, B., Process Engineering Using Object Management Simulation Tools, 20th Annual Quality Assurance Day, Argonne National Laboratory (1996).

Banerjee, B., Women in Science and Technology Program at Argonne National Laboratory, DOE Review of Laboratory Women's Programs, Brookhaven National Laboratory (1996).

Banerjee, B., EPICS Software Distribution, EPICS Collaboration Meeting, CEBAF (1996).

Gluskin, E., "New-Old" Concepts on Insertion Devices, International Workshop on 30-m-long Straight Sections, Himeji, Japan (1996).

Gluskin, E., Some Practical Aspects of Undulator Radiation Properties, SPIE, Denver, Colorado (1996). 
Kuzay, T., (1) X-ray Beam Position Monitor Development, (2) High Heat Load/Flux Engineering and Materials, and (3) APS Front Ends, Himeji, Japan (1996).

Kuzay, T., A Status Report and Review of the APS Front Ends, SPring-8, Japan (1996).

Kuzay, T., Research Partnership between Government, Industry, and Universities: National Laboratory Perspective, American Society of Mechanical Engineers, 1997 Spring Engine Technology Conference, Denver, Colorado (1997).

McNulty, I., X-ray Microscopy at the Advanced Photon Source, Board of Governors Meeting, Argonne National Laboratory (1996).

McNulty, I., X-ray Holography at Third-Generation Storage Rings, IUCr Synchrotron Radiation Satellite Meeting, Argonne National Laboratory (1996).

McNulty, I., Report on SLAC/DESY International Workshop on Interactions of Intense SubPicosecond X-ray Pulses with Matter, Third APS FEL Project Discussion Group, Argonne National Laboratory (1997).

Mills, D.M., Update on Tests of Internally Cooled Si Monochromator Crystal Using Liquid Nitrogen, 3rd Joint APS/ESRF/SPring-8 Workshop, SPring-8 (1996).

Mills, D.M., X-ray Optics Developments at the APS for Third Generation Synchrotron Radiation Sources, IUCr Synchrotron Radiation Satellite Meeting, Argonne National Laboratory (1996).

Rogers, C.S., Experimental Results with Cryogenically Cooled Thin Silicon Crystal X-ray Monochromators on High Heat Flux Beamlines, SPIE, Denver, Colorado (1996).

Rogers, C.S., High heat flux optics at the Advanced Photon Source, Heat Transfer Seminar, Purdue University (1996).

Shenoy, G.K., Status of User Beamlines at the Advanced Photon Source, Third Joint APS/ESRF/SPring-8 Workshop, SPring-8, Japan (1996).

Shenoy, G.K., 7-GeV Advanced Photon Source, The American Society of Mechanical Engineers (1996).

Shenoy, G.K., 7-GeV Advanced Photon Source. A New Tool for the Study of Structure of Materials, The International Conference on Photo-ionization Techniques in Surface Analysis 5, Argonne National Laboratory (1996).

Shenoy, G.K., Science at the Advanced Photon Source, IUCr Synchrotron Radiation Satellite Meeting, Argonne National Laboratory (1996).

Shenoy, G.K., 7-GeV Advanced Photon Source. A New Tool for the Study of Structure of Materials, invited talk, Louisiana State University (1996) 
Shenoy, G.K., First Light at the Advanced Photon Source, Joint APS and AAPT Meeting, Indianapolis, Indiana (1996).

Shenoy, G.K., Early Investigations Using Undulator Radiation at the Advanced Photon Source, Colloqium, University of Illinois, Chicago, Illinois (1997).

Shenoy, G.K., The Advanced Photon Source - A Unique Facility for the Production of High Brilliance X-rays, The American Physical Society March Meeting, Kansas City, Missouri (1997).

Shu, D., Progress of the APS High Heat Load X-ray Beam Position Monitor Development, Illinois Institute of Technology, Chicago, Illinois (1996).

Shu, D., Standard Component Design for Synchrotron Radiation Beamlines, Fudan University, China, and Shanghai Institute for Nuclear Research, China (1996).

Sinha, S.K., Studies of Fluids in Confined Geometry, Colloquium, University of Chicago (1996).

Sinha, S.K., Synchrotron Radiation Studies of Interfaces, Colloquium, Illinois Institute of Technology (1996).

Sinha, S.K., X-ray Studies of Surface Freezing, East-West Conference on Surface Science, Pamporovo, Bulgaria (1996).

Sinha, S.K., X-ray Scattering Studies of Surface Freezing, American Physical Society March Meeting, St. Louis, MO (1996).

Sinha, S.K., Novel Scientific Opportunities at the Advanced Photon Source, Workshop on Synchrotron Radiation Studies of Condensed Matter, Argonne National Laboratory (1996).

Sinha, S.K., X-ray Studies of Surfaces and Interfaces, Workshop on Neutron Reflectivity, Brookhaven National Laboratory (1996).

Sinha, S.K., Grazing Incidence X-ray Studies of Surface Morphology, International Conference on Small-Angle Scattering, Campinas, Brazil (1996).

Sinha, S.K., Synchrotron Radiation Studies of Fluctuating Interfaces, International Conference on Synchrotron Radiation in Materials Science, Illinois Institute of Technology (1996).

Sinha, S.K., Opportunities and Problems with Coherent X-ray Beams in Small Angle Scattering Experiments, International Workshop on 4th Generation SR Sources, DESY, Hamburg, Germany (1996).

Sinha, S.K., Scattering of Partially Coherent Radiation, Colloquium, Physics Dept., University of Kiel, Germany (1996). 
Sinha, S.K., X-ray Studies of Surface and Interface Fluctuations, Colloquium, Bell Laboratories (Lucent Technologies), (1996).

Sinha, S.K., SANS Studies of Sheared Worm-like Micelles, Materials Research Society Fall Meeting, Boston (1996).

Vinokurov, N., Status of the Novosibirsk high power FEL project, Accelerator Systems Division Seminar, Argonne National Laboratory, (1996).

Vinokurov, N., The Integral Equation for the High Gain FEL, LEUTL Physics Meeting, Lawrence Berkeley National Laboratory (1996).

Xu, J., Soft X-ray Programs at the APS, Shanghai Institute of Nuclear Research/Shanghai Synchrotron Radiation Facility (1996).

$\mathrm{Xu}$, J., Research Opportunities on the APS Soft X-ray Spectroscopy Beamline, Synchrotron Radiation Research Center, Taiwan (1996). 
APPENDIX 9 